WHC-SP-1122

Revision 1

UC-900

\title{
Site Support Program Plan for ICF Kaiser Hanford Company
}

ICF Kaiser Hanford Company

Date Published

October 1995

Prepared for the U.S. Department of Energy

Assistant Secretary for Environmental Management

\section{WD Westinghouse P.O Box 1970}

Hanford Company Richland, Washington

Management and Operations Contractor for the

U.S. Depertment of Energy under Contract DE-AC06-87RL10930

DISTRIBUTION OF THIS DOCUMENT IS UNLMITED,

Approved for Public Release

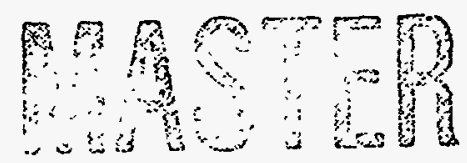




\section{LEGAL DISCLAMER}

This report was prepared as an account of work sponsored by an agency of the United States Government. Neither the United States Government nor any agency thereof, nor any of their employees, nor any of their contractore, subcontractors or their employees, makes any warranty, express or implied, or assumes any legal liability or responsibility for the accuracy, completeness, or any third party's use or the results of such use of any information, apparatus, product, or process disclosed, or represents that its use would not infringe privately owned rights. Reference herein to any specific commercial product, process, or senvice by trade name, trademark, manufacturer, or otherwise, does not necessarily constitute or imply its endorsement, recommendation, or favoring by the United States Government or any agency thereof or its contractors or subcontractors. The views and opinions of authors expressed herein do not necessarily state or reflect those of the United States Government or any agency thereof.

This report has been reproduced from the best available copy. Available in paper copy and microfiche.

Available to the U.S. Department of Energy and its contractors from

U.S. Department of Energy

Office of Scientific and Technical Information (OSTI)

P.O. Box 62

Oak Ridge, TN 37831

(615) 576-8401

Available to the public from the U.S. Department of Commerce National Technical Information Service (NTIS)

5285 Port Royal Road

Springfield, VA 22161

(703) $487-4650$

Printed in the United States of Americe 


\section{DISCLAIMER}

Portions of this document may be illegible in electronic image products. Images are produced from the best available original document. 


\section{RELEASE AUTHORIZATION}

Document Number: WHC-SP-1122, Rev. 1

Document Title: $\quad$ Site Support Program Plan for ICF Kaiser Hanford

Release Date: $\quad 9 / 25 / 95$

This document was reviewed following the procedures described in WHC-CM-3-4 and is:

\section{APPROVED FOR PUBLIC RELEASE}

WHC Information Release Administration Specialist:

(huis isulexifeand)

C. Willingham

$9 / 25 / 95$ 


\section{DISTRIBUTION OF \\ SSPP FOR ICF KAISER HANFORD COMPANY \\ WHC-SP-1122, REVISION 1}

Department

MSIN

Copies

DOE

L. L. Piper

R3-78

S. H. Wisness

A5-18

1

1

WHC

R. E. Traister

B3-63

B3-62

L. J. Hutton

B3-70

L. M. Bogart

A3-88

A3-36

1

1

OSTI

ICF KH
R. L. Benedetti
K. B. Adamson
B. E. Hubbard
P. M. Harrington
M. A. Davis
D. J. Foucault
K. J. Dempsey
D. L. Rittenhouse
M. E. Witherspoon
M. E. Tenvooren
C. E. Anderson

E6-61

E6-61

B4-70

E6-33

E6-34

S2-54

S7-40

G3-07

R3-85

S2-53

E6-28 


\title{
SITE SUPPORT PROGRAM PLAN APPROVAL SHEET
}

\author{
ICF KAISER HANFORD \\ OPERATIONS \& MAINTENANCE \\ W.B.S. 6.5
}

Assistant Manager, Facility Transition
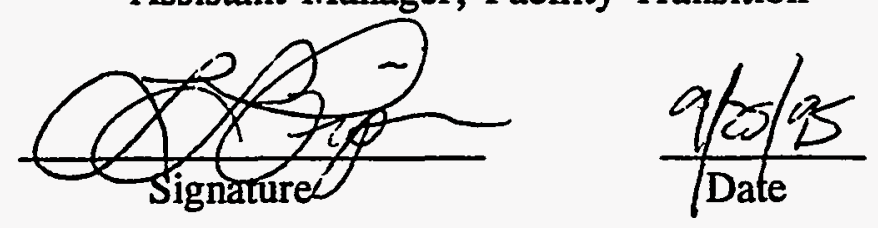

L. L. Piper - DOE-RL

Print Name

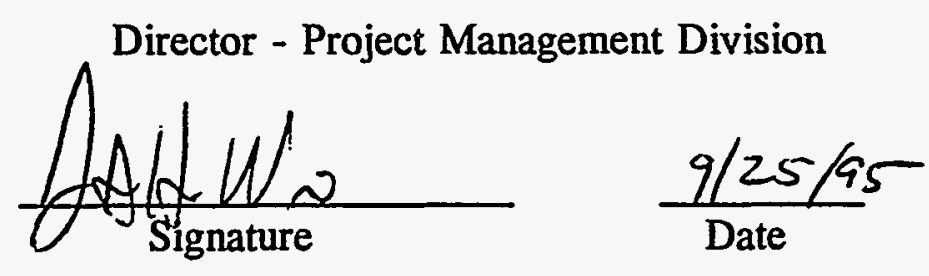

S. H. Wisness - DOE-RL

Print Name

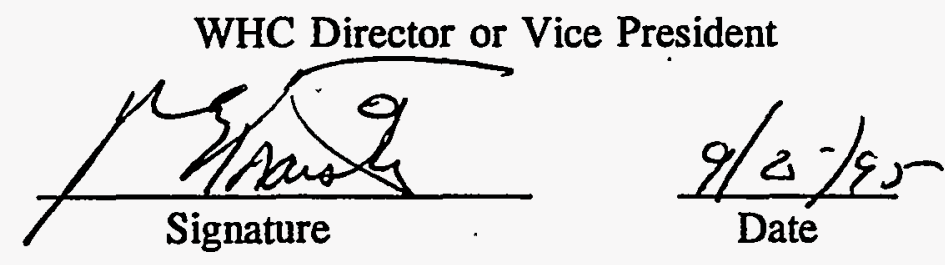

R. E. Traister - WHC

Print Name

ICF Kaiser Hanford Vice President

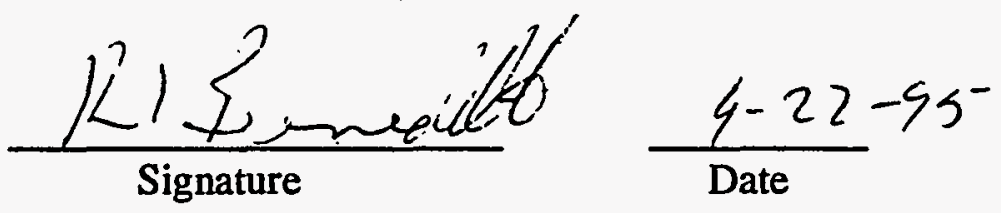

$\frac{\text { R. L. Benedetti - ICF KH }}{\text { Print Name }}$ 
The ICF KH Labor Pools and G\&A include the following:

6.5.1 G\&A Pool - The General and Administrative pool consists of indirect costs of activities which benefits the ICF KH Company and support the Hanford Strategic P1an. The direct costs included within the G\&A pool cannot be identified to a specific cost objective.

6.5.2 CM Pool - The Construction Management Pool consists of costs of activities associated with the placement and administration of Fixed Price Construction contracts, A/E Service Agreements, and miscellaneous specialty contracts.

6.5.3 CF Pool - The Construction Forces Pool consists of costs of activities related to craft training, radiation protection, fabrication shop, hazardous waste assessment, quality control inspections and industrial hygiene.

6.5.4 NM Pool - The Direct Pool - Non Manual Pool consists of costs of activities that are related to the performance of engineering, project management, and construction management services.

Costs that are not included in the above are primarily job specific. They include the following: subcontracts, materials, travel, training, equipment, miscellaneous supplies, purchased services and charges from other contractors. These costs are charged direct to the program/project when incurred. 


\section{INDEX}

\subsection{GENERAL AND ADMINISTRATIVE}

2.0 GENERAL AND ADMINISTRATIVE OVERVIEW (6.5.1)

2.1 Mission

2.2 Assumptions

2.3 Constraints

\subsection{TECHNICAL REQUIREMENT BASELINE}

\subsection{Work Breakdown Structure}

3.2 Work Breakdown

3.2.1 President's Office (6.5.1.1)

3.2.2 Communications (6.5.1.2)

3.2.3 Legal (6.5.1.3)

3.2.4 Human Resources (6.5.1.4)

3.2.5 Organizational Development \& Total Quality Management (6.5.1.5)

3.2.6 Administration (6.5.1.6)

3.2.6.1 Administration Management (6.5.1.6.1)

3.2.6.2 Finance (6.5.1.6.2)

3.2.7 Engineering and Technical Support Services (6.5.1.7)

3.2.7.1 Environmental Programs (6.5.1.7.1)

3.2.7. $\quad$ Industrial Safety and Health Services (6.5.1.7.2)

3.2.7.3 Procedures and Training (6.5.1.7.3)

3.2.8 Environmental, Safety, Health and Quality Performance Assessment (6.5.1.8)

3.2.9 Labor Relations (6.5.1.9)

\subsection{SCHEDULE BASELINE}

4.1 Product Deliverables/Milestone List

5.0 COST BASELINE SUMMARY

$5.1 \quad$ Cost Baseline

5.2 Planned Staffing Profile 


\subsection{GENERAL AND ADMINISTRATIVE POOL (G\&A)}

The General and Administrative pool consists of indirect costs of activities which benefits the ICF Kaiser Hanford Company and supports the Hanford Strategic Plan. The direct costs included within the G\&A pool cannot be identified to a specific cost objective.

\subsection{GENERAL ADMINISTRATIVE POOL OVERVIEW (6.5.1)}

\section{$2.1 \quad$ G\&A Mission/Vision}

The mission of the ICF KH General and Administrative pool is to preserve, upgrade, and operate support services to facilitate the Hanford Site Cleanup mission. Specific services provided by the G\&A pool include President's Office, Communications, Legal, Human Resources, Organizational Development and Total Quality Management, Administration Management, Finance, Environmental Programs, Industrial Safety \& Health, Procedures and Training, Environmental Safety Health and Quality Performance Assessment, and Labor Relations.

ICF KH's vision is that through continued excellent performance, DOE and the Hanford Site will become recognized internationally for environmental research, new technology and scientific development, and cost effective methodology improvements. This will be attained in a safe and healthy working environment for our workers and the public.

\subsection{Assumptions}

The following are key assumptions used to develop goals and strategic (building blocks): Reduction of cost is paramount to success and competitive posturing, outsourcing of work will be conducted on a cost/performance advantage basis, funding and staffing levels will be adequate, reengineering initiatives will be fully supported, award fee and incentive fee criteria will be established, cost savings programs will be supported, work will comply with current regulations and guidance, and the Voluntary Protection Program will continue.

\subsection{Constrainits}

Items that may impede achievements of goals and strategies are as follows: privatization and outsourcing of services, funding support to ICF KH must be adequate for the scope required by DOE, contradictory DOE missions, scheduling/cost software programs changed, prioritization of work due to budget constraints, and continued company reorganizations. 


\section{GENERAL AND ADMINISTRATIVE}

\section{HBS 6.5.1}

FY 1996 Program Plan

\subsection{TECHNICAL REQUIREMENTS BASELINE}

3.1 Work Breakdown Structure

3.2 Work Breakdown 


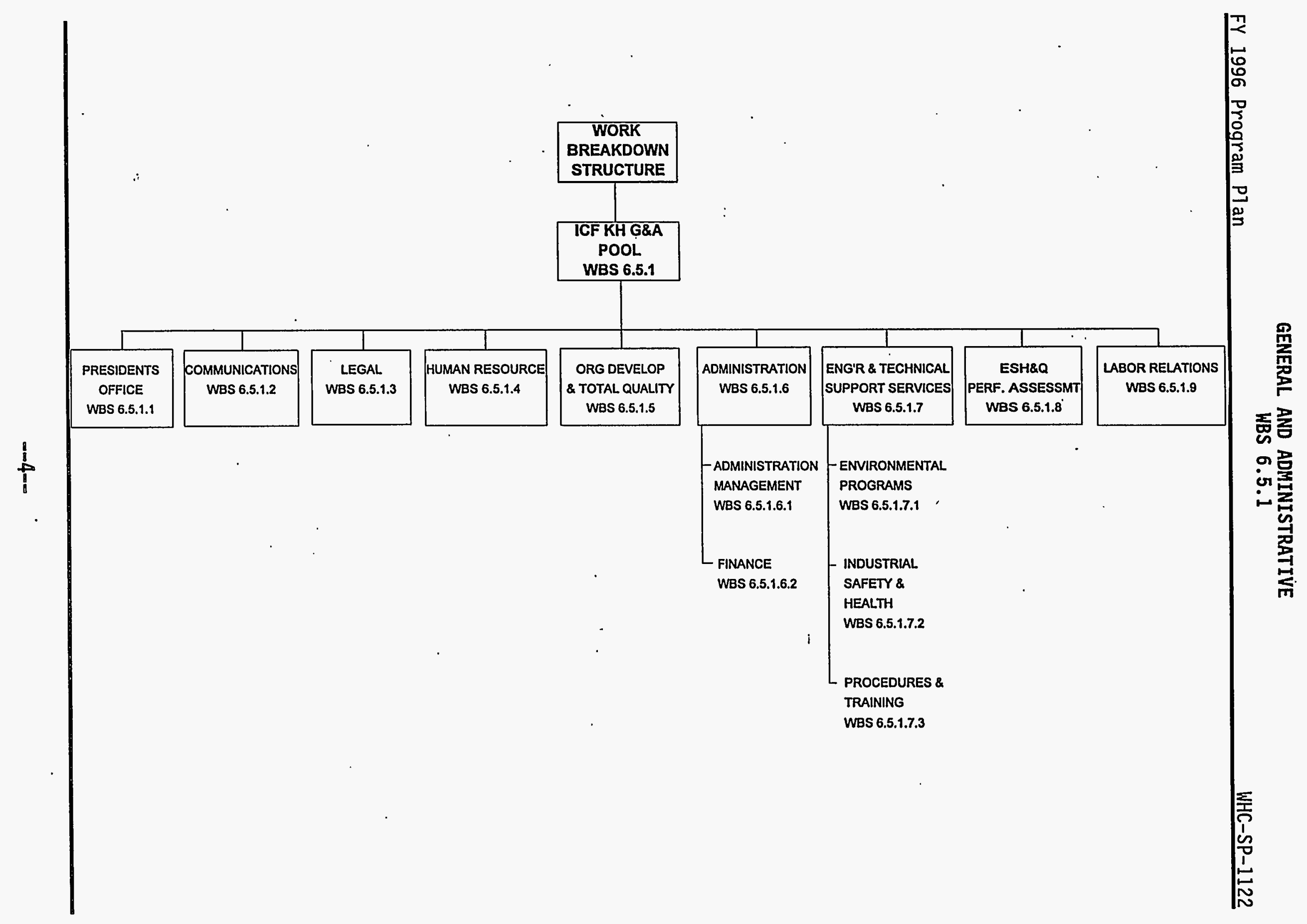


TENERAL AND ADMINISTRATIVE (6.5.1)

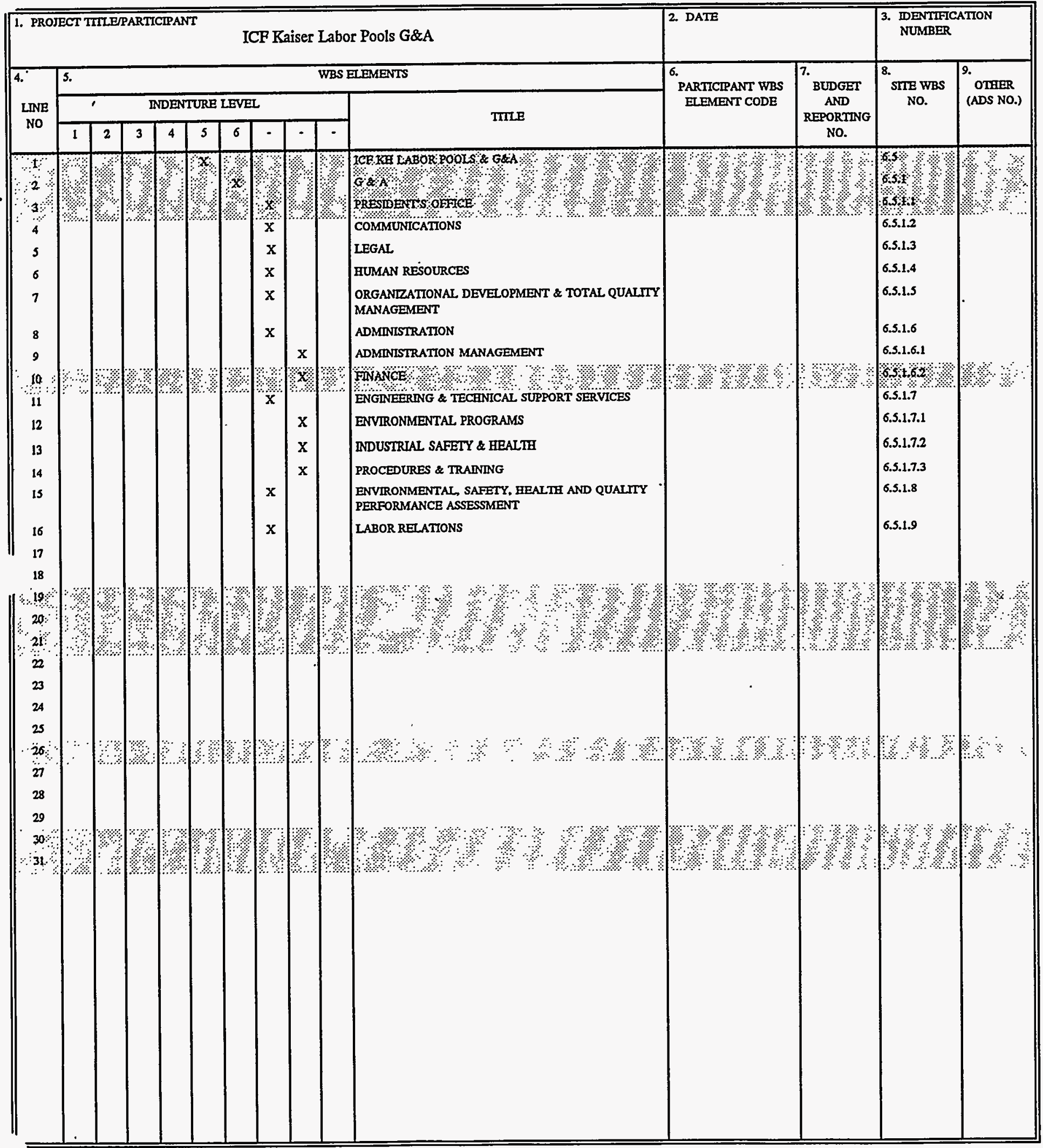




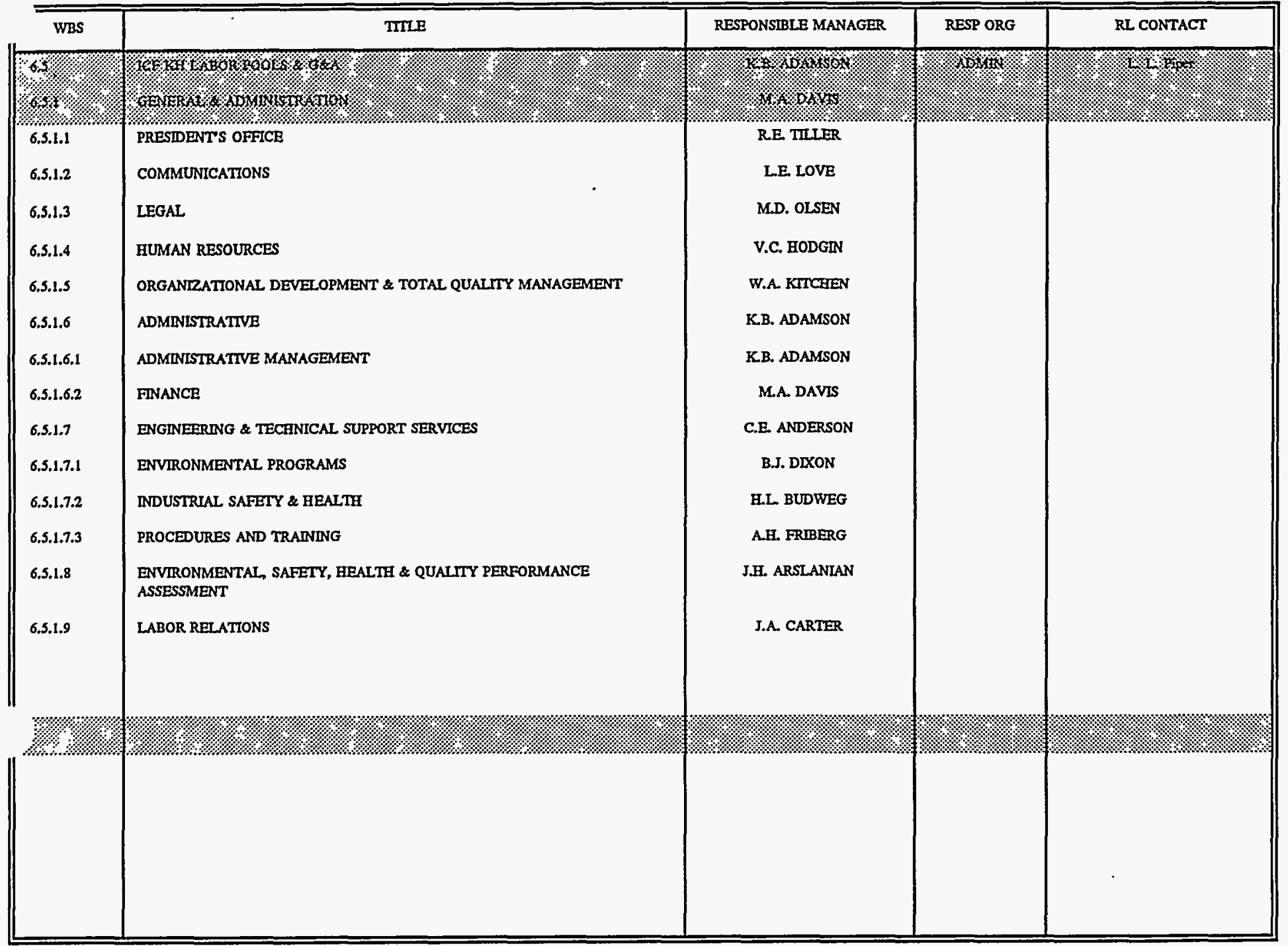




\section{Table 3-1. FY 1996 SSPP GENERAL AND ADMINISTRATIVE WBS Dictionary (6.5.1)}

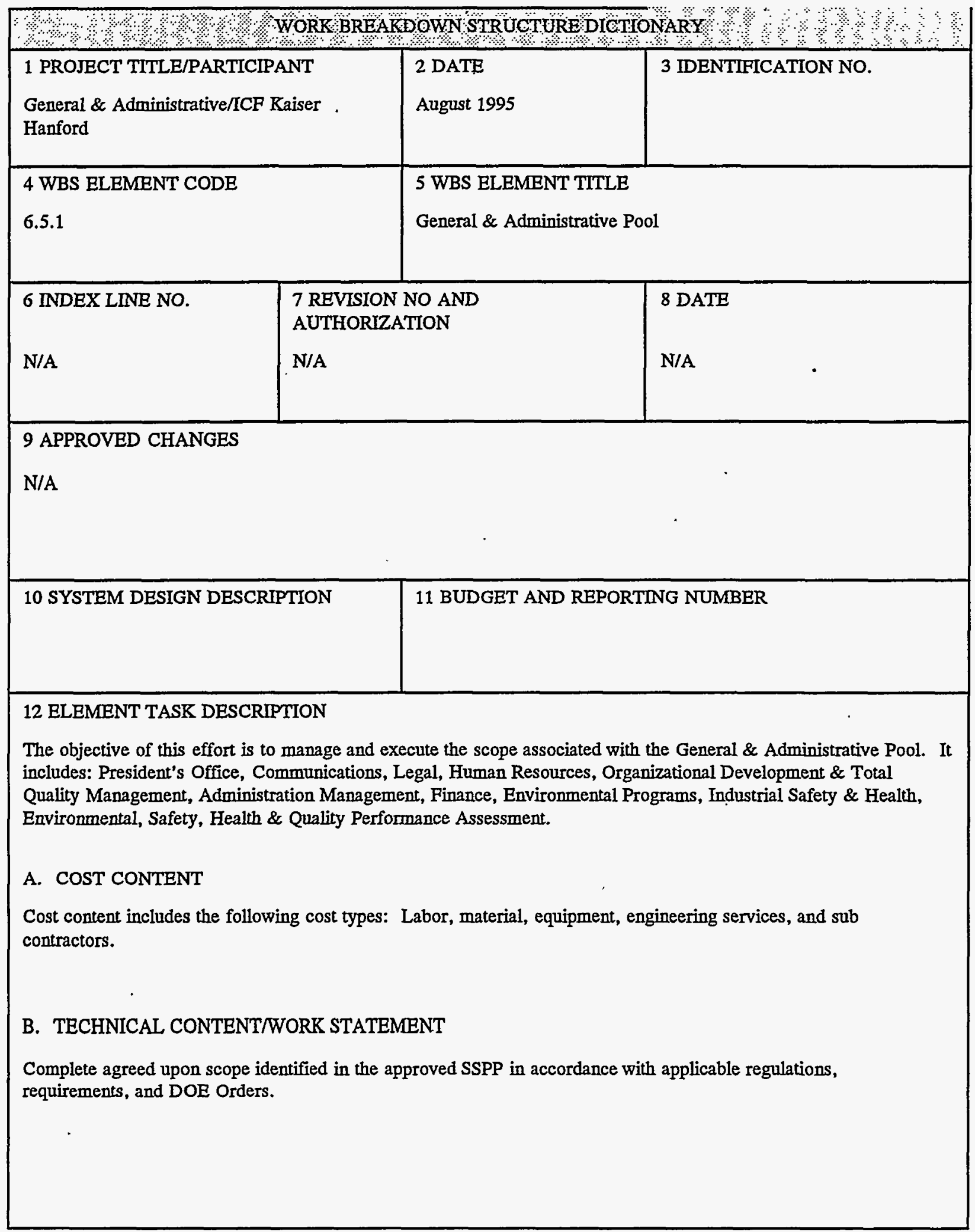




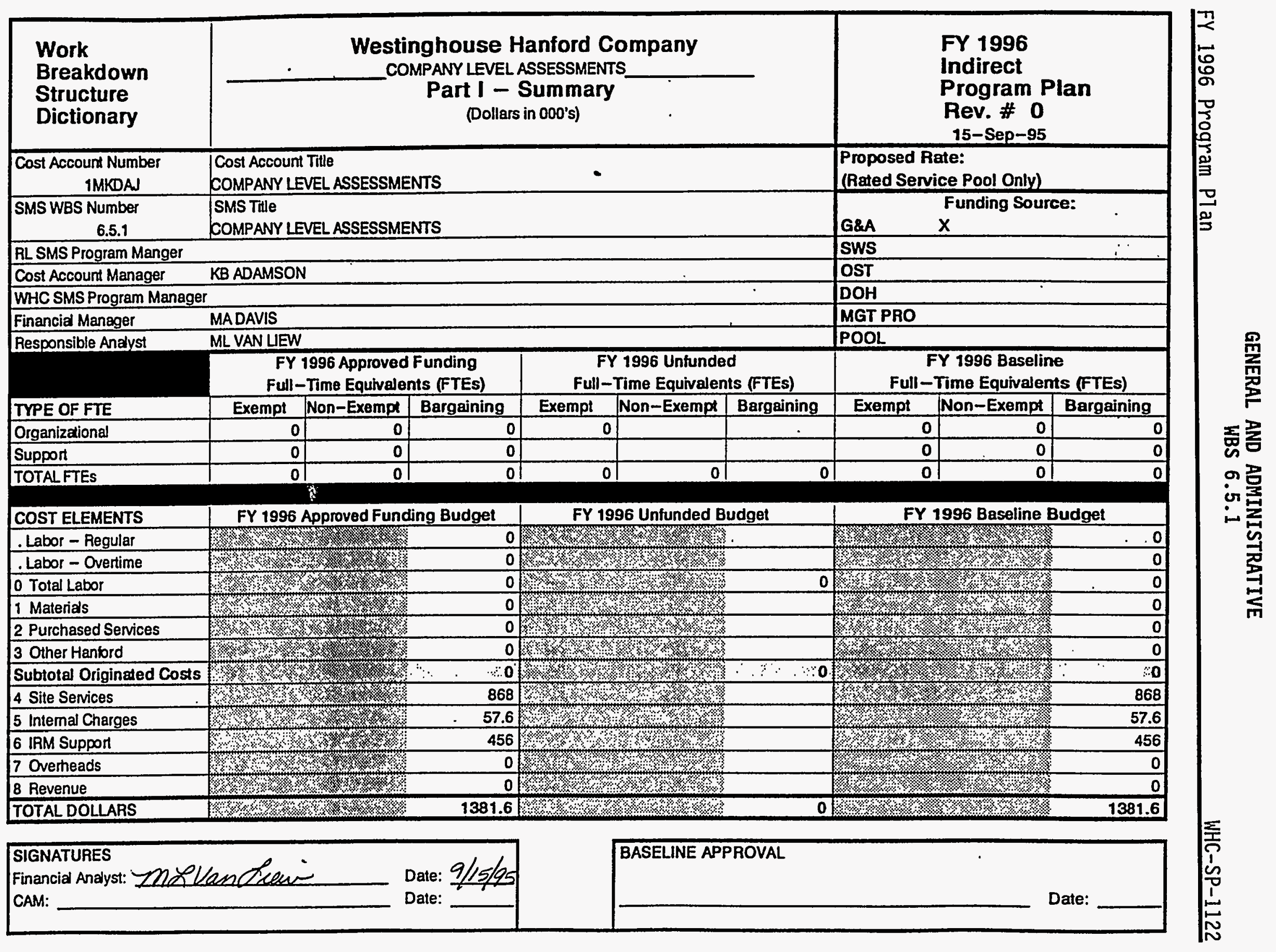




\subsubsection{President's Office (WBS 6.5.1.1)}

Mission

The mission of the Presidents Office is to direct ICF KH in the execution of the scope of work under the RL/ICF KH contract, supplemented by RL directives.

\section{Products/Services Provided}

The President's Office recruits and maintains executive staff to ensure competent and efficient completion of the Hanford and ICF KH Missions. It sets performance standards for executive level employees and ensures standards are met.through training, development, and evaluation of employees. The President's Office also directs the development and implementation of general management philosophy, ICF KH long-range goals and objectives, and ICF KH policy and practices.

Customers

The Primary Customers of the Presidents Office are: U.S. Department of Energy (RL and DOE HQ), Westinghouse Hanford Company, Boeing Computer Services, Pacific Northwest Laboratory, Bechtel Hanford Company, and ICF Kaiser Corporate. 


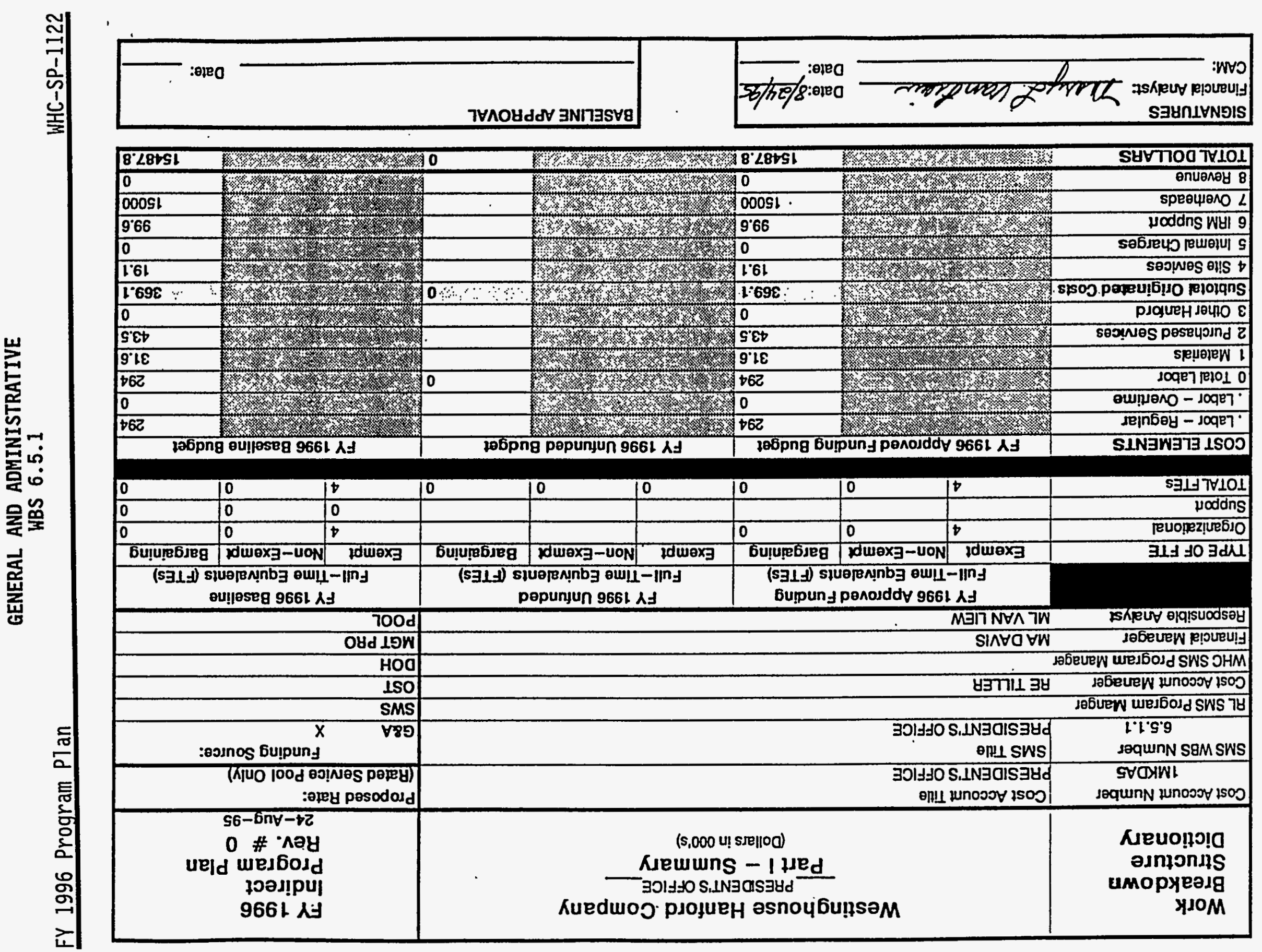




\begin{tabular}{|c|c|c|c|c|}
\hline BUILDING BLOCKS FOR FY 1996 & & & & \\
\hline FY 1996 Building Blocks (5000) & & & & \\
\hline $\begin{array}{l}\text { ORGANIZATIONAL WORK SCOPE DESCRIPTION } \\
\text { (BUDPRES.96 - IMKDA5) }\end{array}$ & $\begin{array}{l}\text { Org. FTEs } \\
\text { in } \\
\text { Workscope }\end{array}$ & $\begin{array}{l}\text { Support } \\
\text { FTEs in } \\
\text { Workscope }\end{array}$ & $\begin{array}{c}\text { FY } 1996 \\
\text { Originated } \\
\text { Cost* }\end{array}$ & $\begin{array}{l}\text { JUSTIFICATION OF SCOPE/IMPACT } \\
\text { IF NOT FUNDED }\end{array}$ \\
\hline $\begin{array}{l}\text { Office of President and General Manager. Contains } \\
\text { General Manager and Deputy with Administrative } \\
\text { Assistant and Executive - Secretary. Provides } \\
\text { direction and guidence at the company level. }\end{array}$ & 4 & & $\$ 369.1 \mathrm{~K}$ & $\begin{array}{l}\text { Detrimental to Business and success of the } \\
\text { compnay in support of the Hanford mission. }\end{array}$ \\
\hline $\begin{array}{l}\text { Assessed Costs: These include Cost Elements } 4 \\
\text { (Services provided by HHC/ICFKH), } 5 \text { (Company level } \\
\text { serivces, ie: Dosimetry), } 6 \text { (IRH Services), and } 7 \\
\text { (Award Fee Estimate). }\end{array}$ & & & $15,118.7$ & \\
\hline LEVEL 1 TOTALS & 4 & & $\begin{array}{r}\$ 369.1 \mathrm{~K} \\
15,118.7 \\
\$ 15,487.8 \mathrm{~K} \\
\end{array}$ & $\begin{array}{lll}\begin{array}{l}\text { Net Originated Cost } \\
\text { Assessed Cost }\end{array} & \text { Target: } 369.0 \\
\text { Total Budget } & & \\
\end{array}$ \\
\hline
\end{tabular}

* Originated Cost $=$ Cost elements $0,1,2,3$

\section{fice of President}

R. Tiller 


\begin{tabular}{|c|c|c|c|c|c|c|c|c|}
\hline \multicolumn{4}{|c|}{ Planned Staffing (Full Time Equivalent) } & \multicolumn{5}{|c|}{ Note: Job Family Only After 1997} \\
\hline Job Famny $\left.{ }^{\circ}\right)^{\circ}$ & Number & \%०? & \%? & ४०. & 2 & (1.2\%: & थ. & $1 .+\%$ ? \\
\hline Job Category & & 1996 & 1997 & 1998 & 1999 & 2000 & 2001 & 2002 \\
\hline MANAGERS & \% & $\%$ & $1 . \% .+4$ & $1.2 \%$ & 1.2 .2 .1 & $12.2 \%$ & $18.2 \%$ & $1.2 \%$ \\
\hline First line & & & & & & & & \\
\hline General/executive & 2 & 2 & 2 & & & & & \\
\hline Projec/Program & & & & & & & & \\
\hline other & & & & & & & & \\
\hline Subtotal & & & & & & & & \\
\hline ENGINEERS: \%?: : & $8 \%$ & $+\%$ & 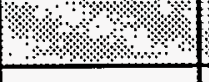 & 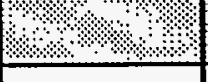 & $1.2 \%$ & 10.2 & 1.2 .2 & $14.2 .1 . \%$ \\
\hline Chemical & & & & & & & & \\
\hline Civil & & & & & & & & \\
\hline Computer & & & & & & & & \\
\hline Electrical & & & & & & & & \\
\hline Environmental & & & & & & & & \\
\hline Industrial & & & & & & & & \\
\hline Mechanical & & & & & & & & \\
\hline Nuclear & & & & & & & & \\
\hline Petroleum/Mining & & & & & & & & \\
\hline Plant & & & & & & & & \\
\hline Quality Control & & - & & & & & & \\
\hline Safety & & & & & & & & \\
\hline Other & & & & & & & & \\
\hline Subtotal & & & & & & & & \\
\hline
\end{tabular}




\begin{tabular}{|c|c|c|c|c|c|c|c|c|}
\hline \multicolumn{9}{|c|}{\begin{tabular}{|l|l|} 
Planned Staffing (Fuli Time Equivalent) & Note: Job Family Only After 1997 \\
\end{tabular}} \\
\hline Job Famitly & Number & 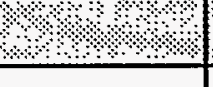 & $2 \%$ & & $\%$ & अ०० & 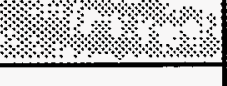 & ४००००० \\
\hline Job Category & & 1996 & 1997 & 1998 & 1999 & 2000 & 2001 & 2002 \\
\hline SCIENTISTS & अ०० & $2 . \bullet+$. & 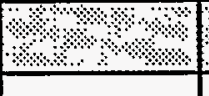 & $2 \%$ & $3 .+4$ & 1.2 .8$. & $2 .+2$ & 12.8. \\
\hline \multicolumn{9}{|c|}{\begin{tabular}{|l|l|l|l|l|l|} 
Chemists & & & & & \\
\end{tabular}} \\
\hline \multicolumn{9}{|l|}{ Environmental } \\
\hline \multicolumn{9}{|l|}{ Geologists } \\
\hline \multicolumn{9}{|l|}{ Life } \\
\hline \multicolumn{9}{|l|}{ Material } \\
\hline \multicolumn{9}{|l|}{ Mathematicians } \\
\hline \multicolumn{9}{|l|}{ Physicists } \\
\hline \multicolumn{9}{|l|}{ Social } \\
\hline \multicolumn{9}{|l|}{ other } \\
\hline \multicolumn{9}{|l|}{ Subtotal } \\
\hline $\begin{array}{l}\text { ADMINISTRATIVE } / 0 \text { THER } \\
\text { PROFESSIONALS }\end{array}$ & $1+\% ?$ & 1.1 .2 .1 .9 & 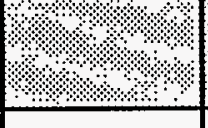 & $2.1 \%$ & $1.1 \%$ & $1.9 .1 \%$ & $1.1 \%$ & 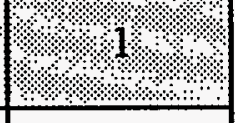 \\
\hline \multicolumn{9}{|c|}{\begin{tabular}{|l|l|} 
Accountant/auditor & \\
\end{tabular}} \\
\hline \multicolumn{9}{|l|}{ Architect } \\
\hline \multicolumn{9}{|l|}{ Buyers/Procurement } \\
\hline Communications & 1 & 1 & 1 & & & & & \\
\hline \multicolumn{9}{|l|}{$\begin{array}{l}\text { Compliance } \\
\text { Inspectors } \\
\end{array}$} \\
\hline \multicolumn{9}{|l|}{$\begin{array}{l}\text { Computer Sys } \\
\text { Analyst }\end{array}$} \\
\hline $\begin{array}{l}\text { Cost Est/ planner/ } \\
\text { sch }\end{array}$ & & & & & & & & \\
\hline
\end{tabular}




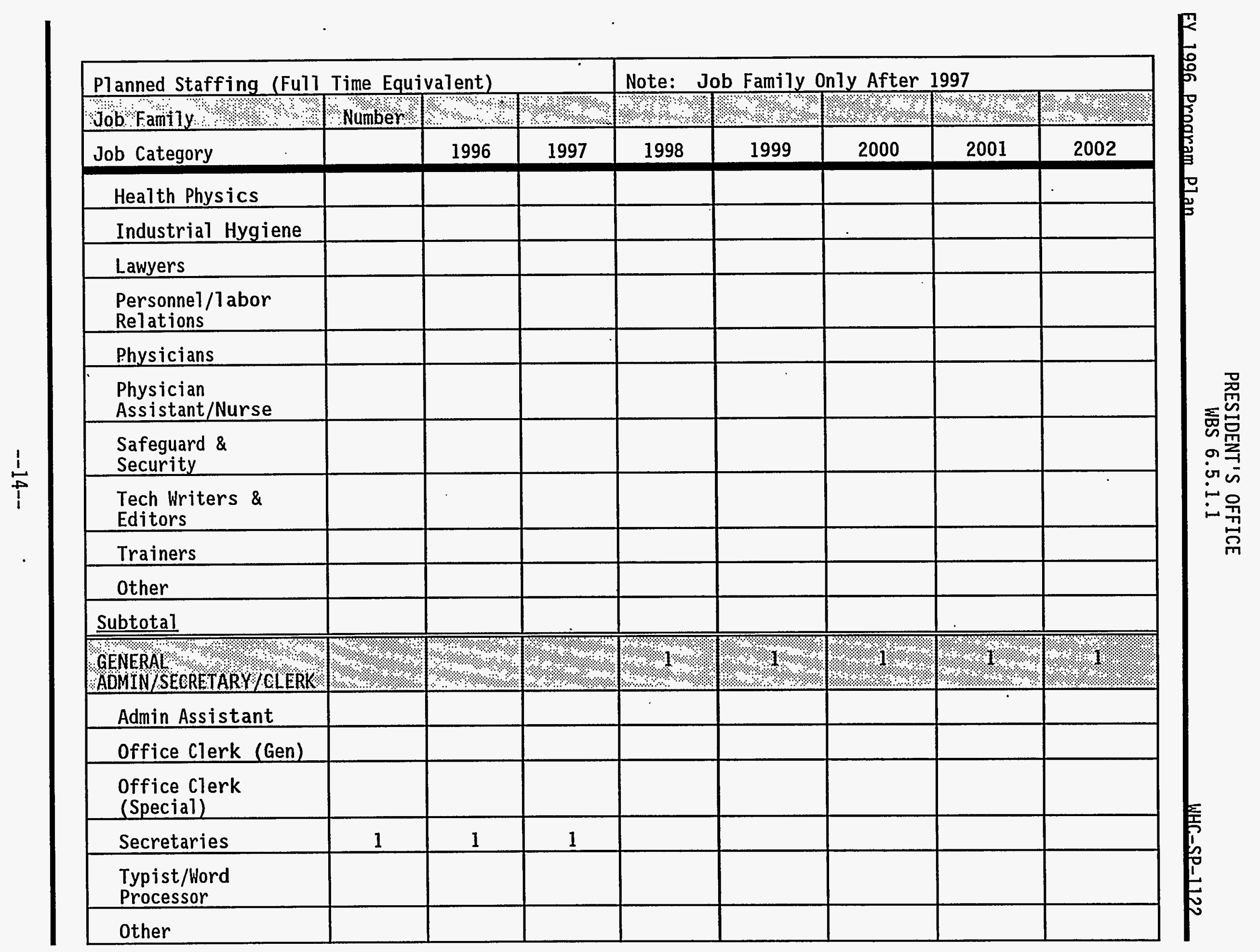




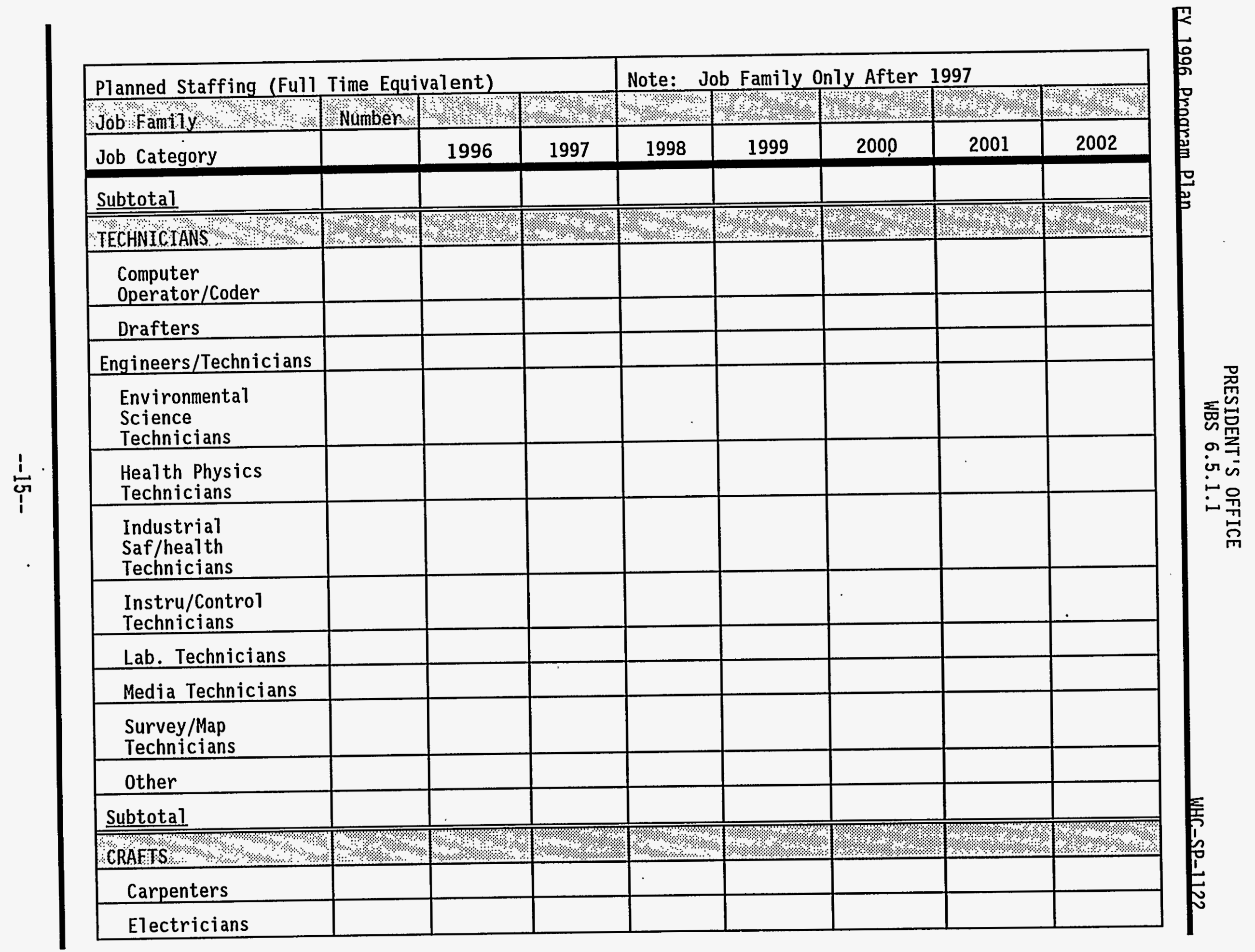




\begin{tabular}{|c|c|c|c|c|c|c|c|c|}
\hline \multicolumn{4}{|c|}{ Planned Staffing (Full Time Equivalent) } & \multicolumn{5}{|c|}{ Note: Job Family Only After 1997} \\
\hline Job Family & Number & \%? & 1.1. & 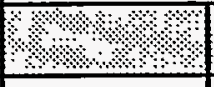 & 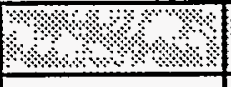 & 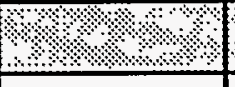 & $2 . .+\%$ & $8 \%$ \\
\hline Job Category & & 1996 & 1997 & 1998 & 1999 & 2000 & 2001 & 2002 \\
\hline \multicolumn{9}{|l|}{ HVAC } \\
\hline \multicolumn{9}{|l|}{ Machinists } \\
\hline \multicolumn{9}{|l|}{ Masons } \\
\hline \multicolumn{9}{|l|}{ Millwrights } \\
\hline \multicolumn{9}{|l|}{ Painters } \\
\hline \multicolumn{9}{|l|}{ Plmbrs/Pipefitters } \\
\hline \multicolumn{9}{|l|}{$\begin{array}{l}\text { Struct/Metal } \\
\text { Workers }\end{array}$} \\
\hline \multicolumn{9}{|l|}{$\begin{array}{l}\text { Vehicl/Mobile } \\
\text { Equip. } \\
\text { Mechanics }\end{array}$} \\
\hline \multicolumn{9}{|l|}{ Welders } \\
\hline \multicolumn{9}{|l|}{ Other } \\
\hline \multicolumn{9}{|l|}{ Subtotal } \\
\hline OPERATORS $\% \%$ & $2 .+\%$ & $\%$ & $1 .+2.9$ & $1.2 . \%$ & 4.4 .8 & $8.1 \%$ & $1 \% .8$. & 19.1 .8 .8 .8 \\
\hline \multicolumn{9}{|l|}{ Lt. Vehicle Drivers } \\
\hline \multicolumn{9}{|l|}{$\begin{array}{l}\text { Material Moving } \\
\text { Equip. }\end{array}$} \\
\hline \multicolumn{9}{|l|}{ Nuclear Plant } \\
\hline \multicolumn{9}{|l|}{$\begin{array}{l}\text { Utilities Waste } \\
\text { Proces }\end{array}$} \\
\hline \multicolumn{9}{|l|}{ other } \\
\hline Subtotal & & & & & & & & \\
\hline
\end{tabular}




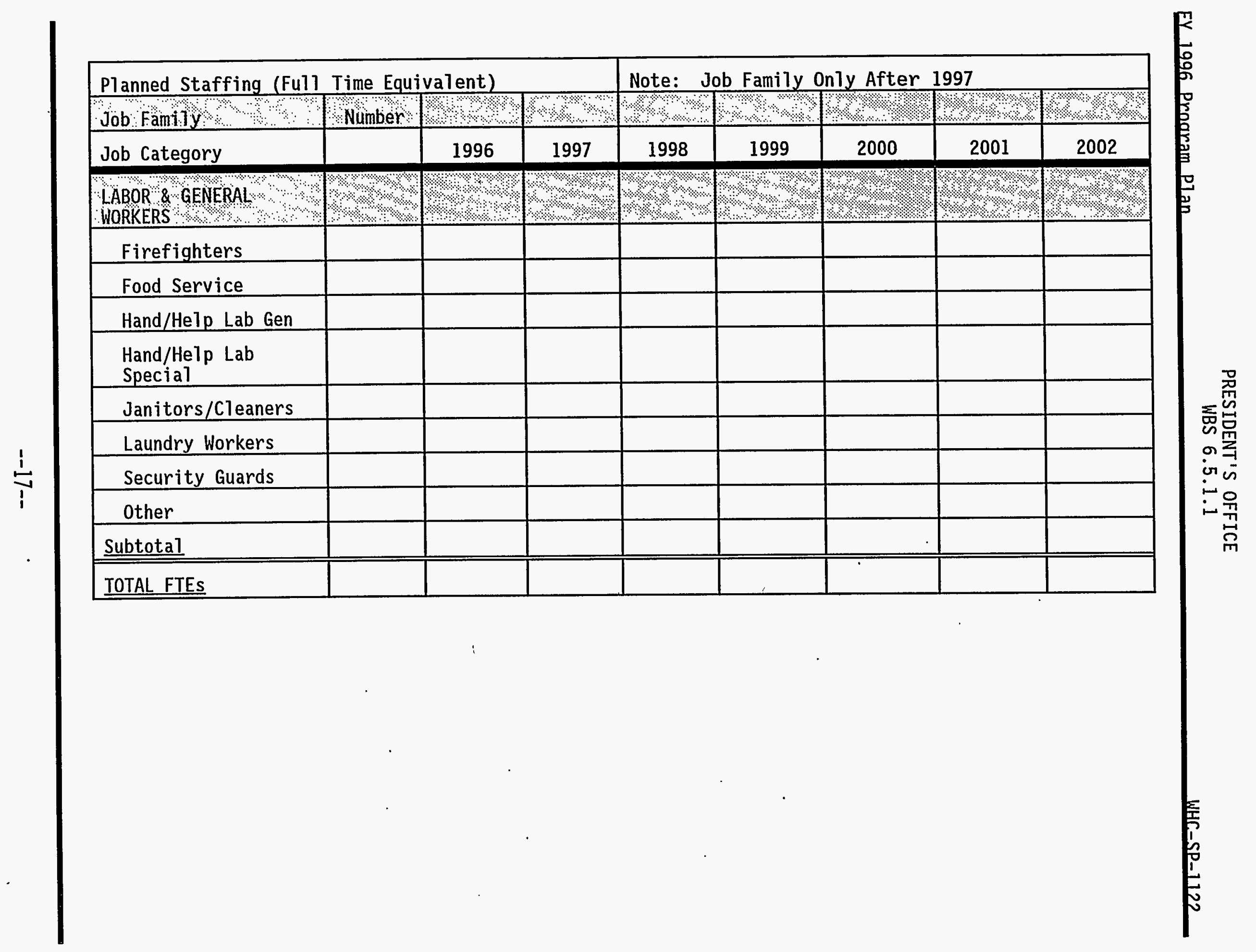




\subsubsection{Communications (WBS 6.5.1.2)}

\section{$\underline{\text { Mission }}$}

The mission of the Office of Communications is to coordinate and manage all communications for the company. Communications gathers, verifies, and disseminates information within ICF $\mathrm{KH}$ and to the public.

\section{Products/Services Provided}

The Products and Services performed by the Communications Office.

- Internal Communications

- The Reach

- Across the Wire

- All Employee Messages

- Management Bulletins

- All Employee and Special Events

- External Communications

- News releases

- Response to inquires

- Editorial board meetings

- News Conferences

- Community Relations

- Corporate Contributions

- CBC Scholarship/Mentor Program

- WSU Branch Campus Grant

- Northwest Public TV

- United Way

- Savings Bonds

- Speakers Bureau

- etc.

- Corporate Communications/Relations

- Press Releases

- ICF Update

- Management Messages

- Corporate Visitors/Tours

\section{Customers}

The primary customers of the Communications Office are: ICF Kaiser Hanford Employees, ICF Kaiser (Corporate), Westinghouse Hanford Company, Local/National News Media, Local communities and U.S. Department of Energy (RL \& HQ). 


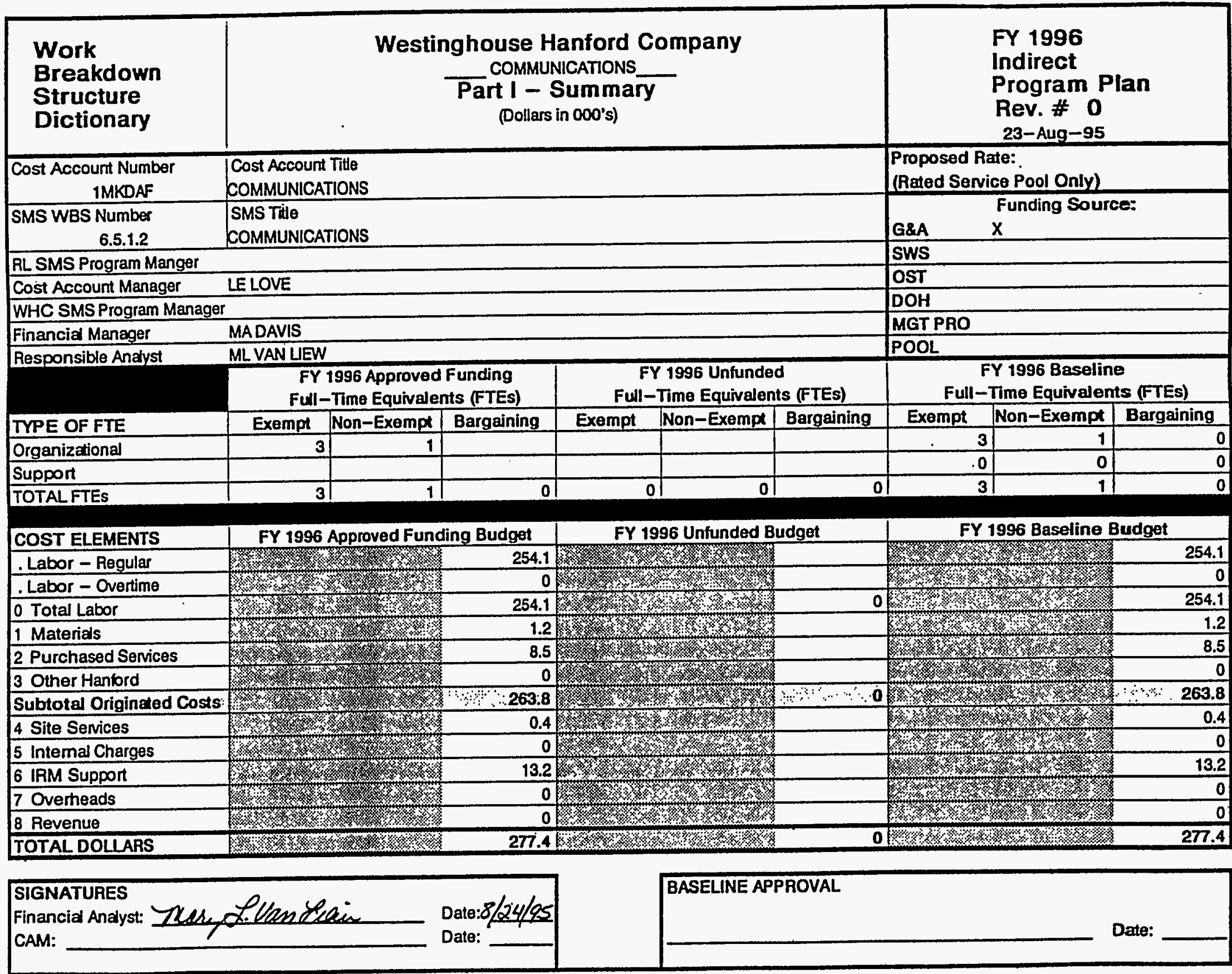


OFFICE OF COMMUNICATIONS

L.E. Love

BUILDING BLOCKS FOR FY 1996

\begin{tabular}{|c|c|c|c|c|}
\hline $\begin{array}{l}\text { OREANIZATIOHAL WORK SCDPE DESCRIPTION } \\
\text { (96-BLOGE - MKKOAF) }\end{array}$ & $\begin{array}{l}\text { Org. FTEs } \\
\text { in } \\
\text { Workscope }\end{array}$ & $\begin{array}{l}\text { Support } \\
\text { FTEs in } \\
\text { Morkscope }\end{array}$ & $\begin{array}{l}\text { Fy } 1996 \\
\text { Originated } \\
\text { Cost* }\end{array}$ & $\begin{array}{l}\text { JUSTIFICATION OF SCOPE/IMPACT } \\
\text { IF NOT FUNDED }\end{array}$ \\
\hline $\begin{array}{l}\text { Internal Cominnications } \\
\text { The Reach } \\
\text { Across the Wire } \\
\text { All employee messages } \\
\text { Managenent bullesins } \\
\text { All employee \& special events } \\
\text { official company bulletin boards } \\
\text { other cost Elements included: }(1,2,3)\end{array}$ & 1.6 & & $\begin{array}{l}\$ 77.7 \\
\$ 9.7\end{array}$ & $\begin{array}{l}\text { - Poor comminication w/employees affects } \\
\text { productivity } \\
\text { - Hanage required state/federal postings } \\
\text { - Detrimental impact to company image w/employees } \\
\text { - Lack of employee support/involvement toward } \\
\text { success of company }\end{array}$ \\
\hline $\begin{array}{l}\text { External Communications } \\
\text { News releases } \\
\text { Response to inquiries } \\
\text { Edi torial Board meet ings } \\
\text { News Conferences }\end{array}$ & .50 & & $\$ 36.8$ & $\begin{array}{l}\text { - Detrimental impact to company image } w / \text { the } \\
\text { public } \\
\text { - No community support } \\
\text { - Hegative impact to local, state, and national } \\
\text { political support }\end{array}$ \\
\hline $\begin{array}{l}\text { Community Relations } \\
\text { CBC Scholarship/Mentor progran } \\
\text { WSU Branch Canpus Grant } \\
\text { Horthwest Public TV } \\
\text { TRIDEC activiries } \\
\text { Sunfest Children's Festival } \\
\text { HalkAmerica } \\
\text { United May } \\
\text { Savings Bonds } \\
\text { Technical Symposiums } \\
\text { Tracking/Reporting/Publicizing Community Outreach } \\
\text { octivities } \\
\text { Tours } \\
\text { Speakers Bureau } \\
\text { Shadowing Program - and other outreach to schools } \\
\text { Tri-city Education Consortium } \\
\text { Corporate donations response point } \\
\text { Exhibits and Brochures }\end{array}$ & 1.50 & & 5110.2 & $\begin{array}{l}\text { - Detrimental impact to company image w/commity } \\
\text { leaders } \\
\text { - Ho commenity support } \\
\text { - Detrimental impact to companies educational } \\
\text { outreach } \\
\text { - Ho support from edueational institutions } \\
\text { - Improves employee morale }\end{array}$ \\
\hline
\end{tabular}


OFFICE OF COMMUNICATIONS

L.E. Love

\begin{tabular}{|c|c|c|c|c|}
\hline \multicolumn{5}{|l|}{ BUILDING BLOCKS FOR FY 1996} \\
\hline \multicolumn{5}{|l|}{ Fy 1996 Building Blocks $(\$ 000)$} \\
\hline $\begin{array}{l}\text { ORGAHIZATIONAL WORK SCOPE DESCRIPTION } \\
\text { (96-8UDGE - 1MKDAF) }\end{array}$ & $\begin{array}{l}\text { Org. FTEs } \\
\text { in } \\
\text { Workscope }\end{array}$ & $\begin{array}{l}\text { Support } \\
\text { FTEs in } \\
\text { Workscope }\end{array}$ & $\begin{array}{l}\text { FY } 1996 \\
\text { Originated } \\
\text { Cost" }\end{array}$ & $\begin{array}{l}\text { JUSTIFICATION OF SCOPE/IMPACT } \\
\text { IF NOT FUNDED }\end{array}$ \\
\hline $\begin{array}{l}\text { Corporate Comminications/Relations } \\
\text { ICF Update } \\
\text { Management message } \\
\text { Press Releases } \\
\text { Corporate visitors \& tours }\end{array}$ & .4 & & $\$ 29.4$ & - Detrimental impact to corporate relations \\
\hline $\begin{array}{l}\text { Assessed Costs: These include Cost Elements } 4 \\
\text { (Services provided by UHC/ICFKH), } 5 \text { (Company level } \\
\text { serveies, ie: Dosimetry), } 6 \text { (IRM Services), and } 7 \\
\text { (DOH adders applied to support from Other (abor). }\end{array}$ & & & $\$ 13.6$ & . \\
\hline LEVEL 1 TOTALS & 4 & & 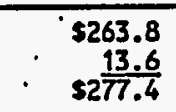 & $\begin{array}{l}\text { Het Originated Cost } \\
\text { Assessed Costs } \\
\text { Total Budget }\end{array}$ \\
\hline
\end{tabular}

* Originated Cost $=$ Cost elements $0,1,2,3-$ Dollars in thousands 


\begin{tabular}{|c|c|c|c|c|c|c|c|c|}
\hline \multicolumn{4}{|c|}{ Planned Staffing (Full Time Equivalent) } & \multicolumn{5}{|c|}{ Note: Job Family Only After 1997} \\
\hline Job Family & Number & 2.2 & 2.2 & 202 & 2:2 & 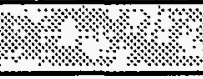 & 12:2: & 2.2 .21 \\
\hline Job Category & & 1996 & 1997 & 1998 & 1999 & 2000 & 2001. & 2002 \\
\hline MANAGERS. & 2010 & 2.2.2. & 20 & 2.11 & 2.12 & -112 & 8.1 & 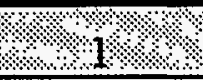 \\
\hline First line & & & & & & & & \\
\hline General/executive & 1 & 1 & 1 & & & & & \\
\hline Projec/Program & & & & & & & & \\
\hline other & & & & & & & & \\
\hline Subtotal & & & & & & & & \\
\hline ENGINEERS & 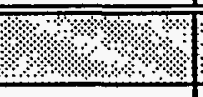 & ॠण. & (2) & $12 \div$ & 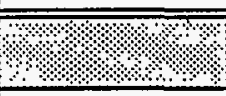 & 2 & 10 & $\overline{2.28 .20}$ \\
\hline Chemical & & & & & & & & \\
\hline Civil & & & & & & & & \\
\hline Computer & & & & & & & & \\
\hline Electrical & & & & & & & & \\
\hline Environmental & & & & & & & & \\
\hline Industrial & & & & & & & & \\
\hline Mechanical & & & & & & & & \\
\hline Nuclear & & & & & & & & \\
\hline Petroleum/Mining & & & & & & & & \\
\hline Plant & & & & & & & & \\
\hline Quality Control & & & & & & & & \\
\hline Safety & & & & & & & & \\
\hline Other & & & & & & & & \\
\hline Subtotal & & & & & & & & \\
\hline
\end{tabular}




\begin{tabular}{|c|c|c|c|c|c|c|c|c|}
\hline \multicolumn{4}{|c|}{ Planned Staffing (Full Time Equivalent) } & \multicolumn{5}{|c|}{ Note: Job Fami.7y Only After 1997} \\
\hline Job famfuy & Nümber & ४४४। & $8 \%$ & $2 .+\%$ & \%०००: & (2) & 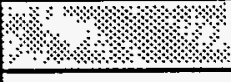 & $1 \%$ \\
\hline Job Category & & 1996 & 1997 & 1998 & 1999 & 2000 & 2001 & 2002 \\
\hline SGIENTISTS & $1 .+2$. & $8 \%$ & 1.2 .1 & 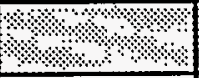 & 3.2. & 12.8 & \%. & 1 \\
\hline Chemists & & & & & & & & \\
\hline Environmenta] & & & & & & & & \\
\hline Geologists & & & & & . & & & \\
\hline Life & & & & & & & & \\
\hline Material & & & & & & & & \\
\hline Mathematicians & & & & & & & & \\
\hline Physicists & & & & & & & & \\
\hline Social & & & & & & & & \\
\hline Other & & & & & & & & \\
\hline Subtotal & & & & & & & & \\
\hline $\begin{array}{l}\text { ADMINISTRATIVE } / 0 \text { THER } \\
\text { PROFESSIONALS }\end{array}$ & & 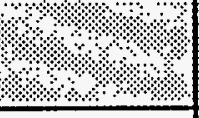 & 1.1 .1 .2$. & $8.2 \%$ & 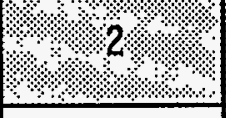 & 1.82 .8 & 14.2 .2$. & 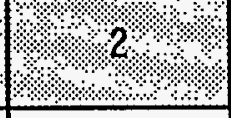 \\
\hline Accountant/auditor & & & & & & & & \\
\hline Architect & & & & & & & & \\
\hline Buyers/Procurement & & & & & & & & \\
\hline Communications & 2 & 2 & 2 & & & & & \\
\hline $\begin{array}{l}\text { Compl iance } \\
\text { Inspectors }\end{array}$ & & & & & & & & \\
\hline $\begin{array}{l}\text { Computer Sys } \\
\text { Analyst }\end{array}$ & & & & & & & & \\
\hline $\begin{array}{l}\text { Cost Est/ planner/ } \\
\text { sch }\end{array}$ & & & & & & & & \\
\hline
\end{tabular}




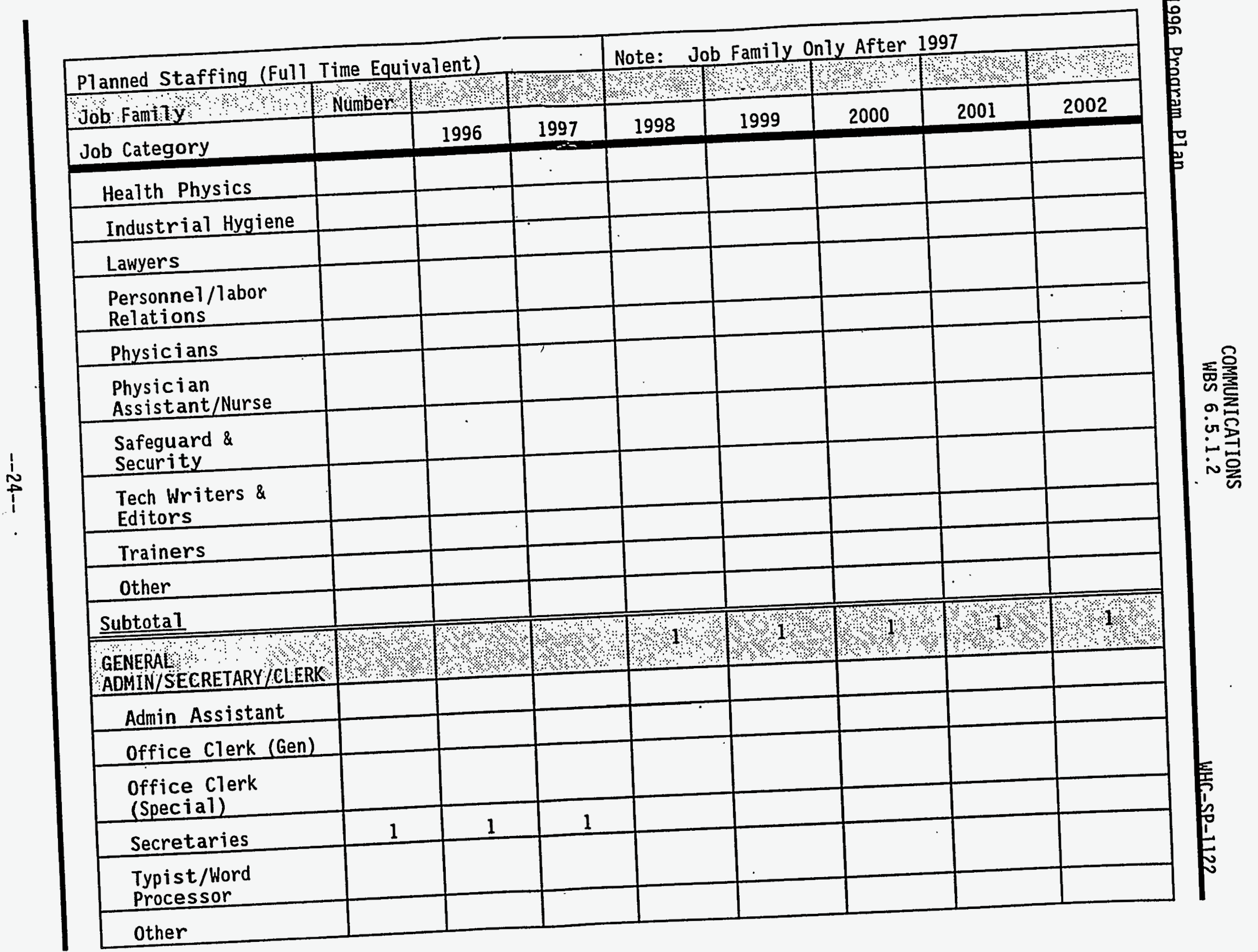




\begin{tabular}{|c|c|c|c|c|c|c|c|c|}
\hline \multicolumn{9}{|c|}{ Note: Job Family Only After 1997} \\
\hline Job Family y & Number. & ans & अ, & + & ४ै। & ४ै। & ४ै।? & अें \\
\hline Job Category & & 1996 & 1997 & 1998 & 1999 & 2000 & 2001 & 2002 \\
\hline \multicolumn{9}{|l|}{ Subtotal } \\
\hline \multicolumn{9}{|c|}{ 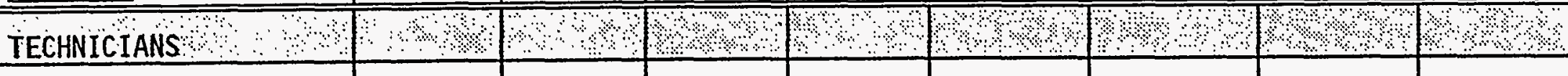 } \\
\hline \multicolumn{9}{|l|}{$\begin{array}{l}\text { Computer } \\
\text { Operator/Coder }\end{array}$} \\
\hline \multicolumn{9}{|l|}{ Drafters } \\
\hline \multicolumn{9}{|l|}{ Engineers/Technicians } \\
\hline \multicolumn{9}{|l|}{$\begin{array}{l}\text { Environmental } \\
\text { Science } \\
\text { Techinicians } \\
\end{array}$} \\
\hline \multicolumn{9}{|l|}{$\begin{array}{l}\text { Health Physics } \\
\text { Technicians }\end{array}$} \\
\hline \multicolumn{9}{|l|}{$\begin{array}{l}\text { Industrial } \\
\text { Saf/health } \\
\text { Technicians } \\
\end{array}$} \\
\hline \multicolumn{9}{|l|}{$\begin{array}{l}\text { Instru/Control } \\
\text { Technicians }\end{array}$} \\
\hline \multicolumn{9}{|l|}{ Lab. Technicians } \\
\hline \multicolumn{9}{|l|}{ Media Technicians } \\
\hline \multicolumn{9}{|l|}{$\begin{array}{l}\text { Survey/Map } \\
\text { Technicians }\end{array}$} \\
\hline \multicolumn{9}{|l|}{ Other } \\
\hline \multicolumn{9}{|l|}{ Subtotal } \\
\hline CRAFTS & 3 & लि? & २४ & ४०० & ॥ै। & अ०। & $\$$ & + \\
\hline \multicolumn{9}{|l|}{ Carpenters } \\
\hline Electricians & & & & & & & & \\
\hline
\end{tabular}




\begin{tabular}{|c|c|c|c|c|c|c|c|c|}
\hline \multicolumn{4}{|c|}{ Planned Staffing (Full Time Equivalent) } & \multirow{2}{*}{\multicolumn{5}{|c|}{ Note: Job Family Only After 1997}} \\
\hline Job Family ${ }^{2}$ \% & Number & \%?.. & 82 & \%. & & & & \\
\hline Job Category & & 1996 & 1997 & 1998 & 1999 & 2000 & 2001 & 2002 \\
\hline \multicolumn{9}{|l|}{ HVAC } \\
\hline \multicolumn{9}{|l|}{ Machinists } \\
\hline \multicolumn{9}{|l|}{ Masons } \\
\hline \multicolumn{9}{|l|}{ Millwrights } \\
\hline \multicolumn{9}{|l|}{ Painters } \\
\hline \multicolumn{9}{|l|}{ P1mbrs/Pipefitters } \\
\hline \multicolumn{9}{|l|}{$\begin{array}{l}\text { Struct/Metal } \\
\text { Workers }\end{array}$} \\
\hline \multicolumn{9}{|l|}{$\begin{array}{l}\text { Vehicl/Mobile } \\
\text { Equip. } \\
\text { Mechanics } \\
\end{array}$} \\
\hline \multicolumn{9}{|l|}{ Welders } \\
\hline \multicolumn{9}{|l|}{ other } \\
\hline \multicolumn{9}{|l|}{ Subtotal } \\
\hline \multicolumn{9}{|c|}{ 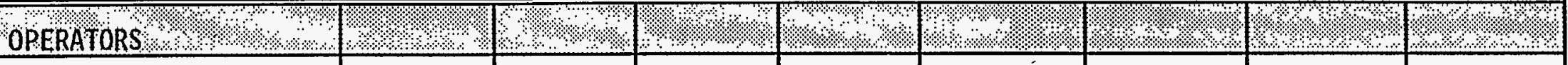 } \\
\hline \multicolumn{9}{|l|}{ Lt. Vehicle Drivers } \\
\hline \multicolumn{9}{|l|}{$\begin{array}{l}\text { Material Moving } \\
\text { Equip. }\end{array}$} \\
\hline \multicolumn{9}{|l|}{ Nuclear Plant } \\
\hline \multicolumn{9}{|l|}{$\begin{array}{l}\text { Utilities Waste } \\
\text { Proces }\end{array}$} \\
\hline \multicolumn{9}{|l|}{ Other } \\
\hline Subtotal & & & & & & & & \\
\hline
\end{tabular}




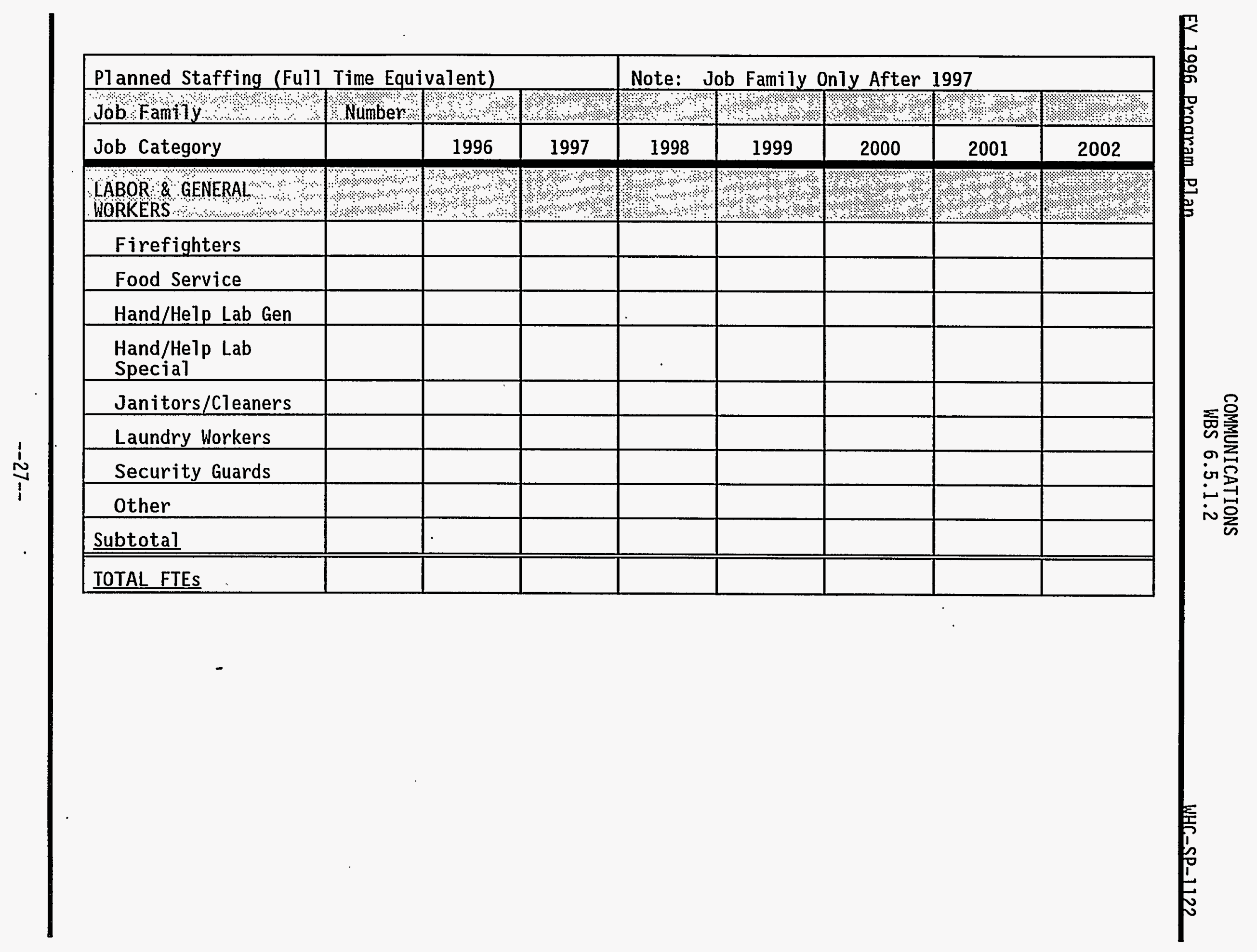




\subsubsection{Legal (WBS 6.5.1.3)}

\section{$\underline{\text { Mission }}$}

The mission of the Law Department is to help insure that ICF Kaiser Hanford's activities are consistent with applicable legal and contractual requirements and obligations.

\section{Products/Services Provided}

The primary services rendered by the Legal Department are:

- Legal consultation with senior management of ICF KH, Corporate Management and all other personnel of ICF KH.

- Performance of internal audits and surveillances of activities under ICF KH's purview to ensure financial practices and management programs are in accordance with federal regulations, company procedures and terms.

\section{Primary Customers}

The primary customers of the Legal Department are: ICF Kaiser Hanford, ICF Kaiser (Corporate), Westinghouse Hanford Company, U.S. Department of Energy (RL \& HQ) and outside contractors. 


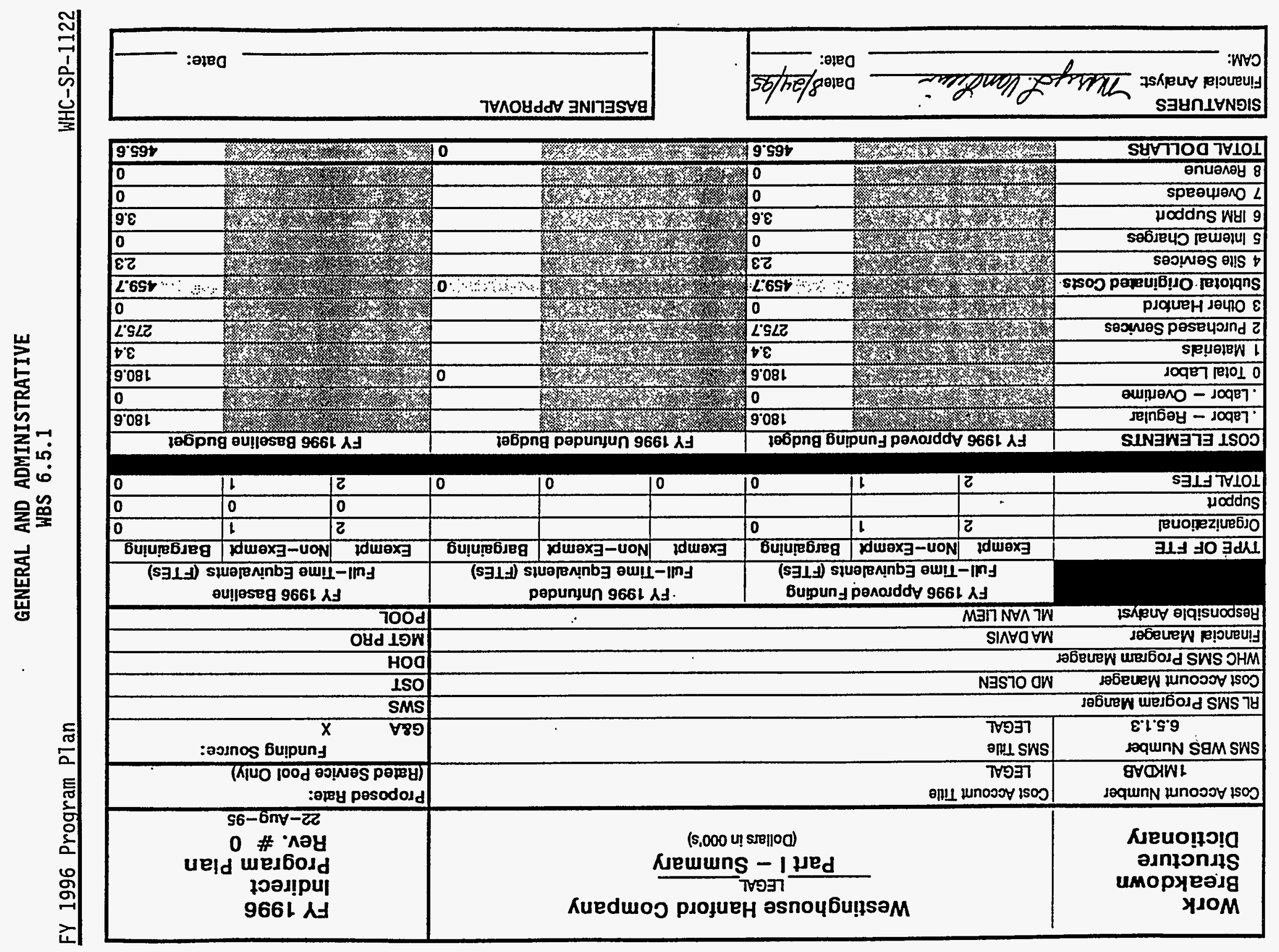


LEGAL

* Originated Cost $=$ Cost elements $0,1,2,3$

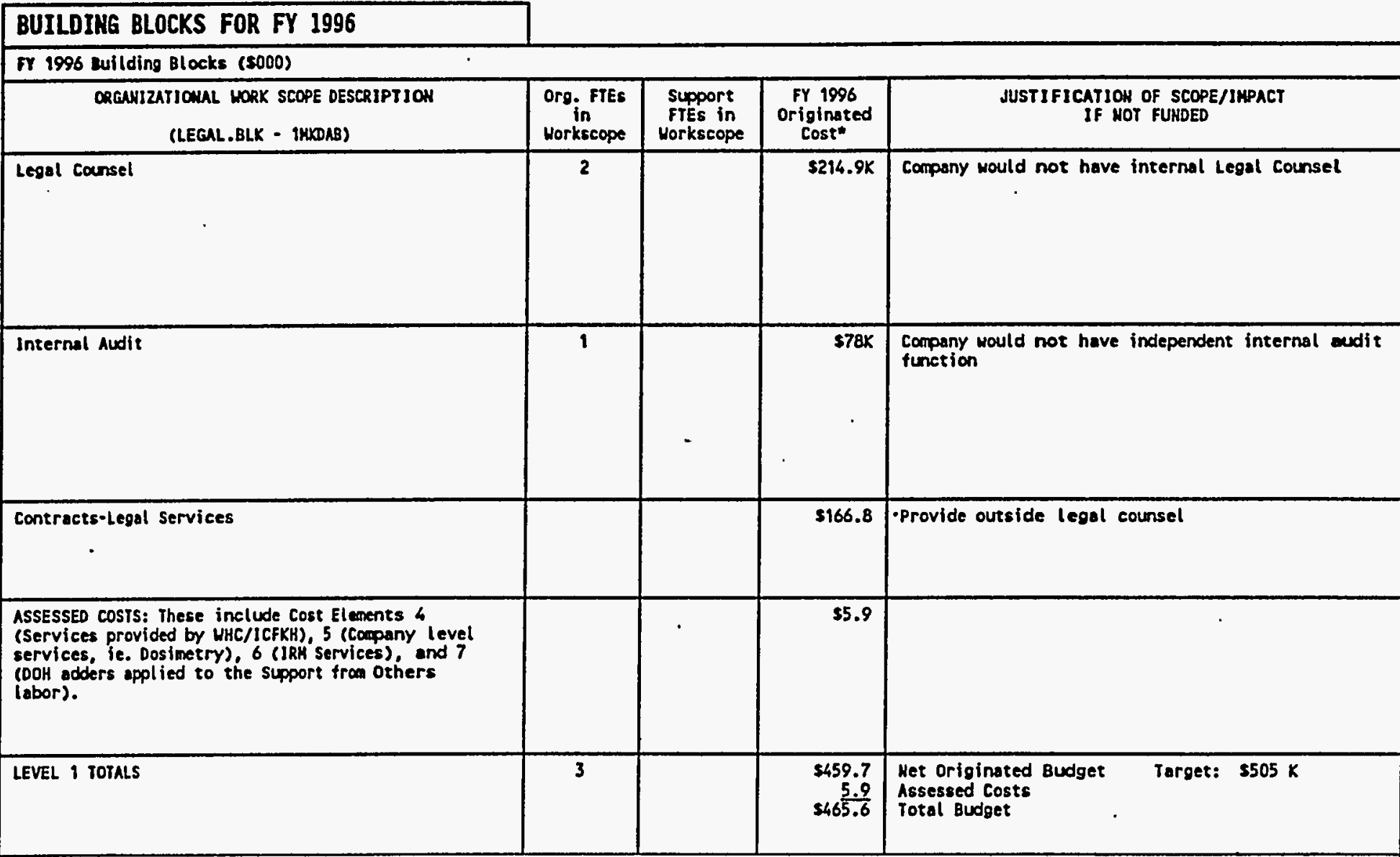




\begin{tabular}{|c|c|c|c|c|c|c|c|c|}
\hline \multicolumn{4}{|c|}{ Planned Staffing (Full Time Equivalent) } & \multicolumn{5}{|c|}{ Note: Job Family Only After 1997} \\
\hline Job Family & Number: & अ? & 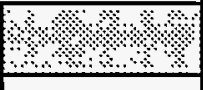 & 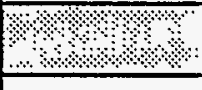 & 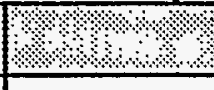 & 1.2\% & थथ थ थ & 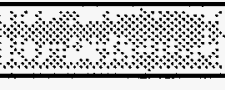 \\
\hline Job Category & & 1996 & 1997 & 1998 & 1999 & 2000 & 2001 & 2002 \\
\hline MANAGERS & 2 & 2 & 2 & $2.1 \%$ & $\$ .1 .2$ & 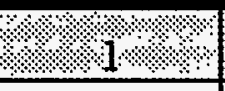 & $2 \% 1$ & 2.11 .2 \\
\hline First 7 ine & & & & & & & & \\
\hline General/executive & 1 & 1 & 1 & & & & & \\
\hline Projec/Program & & & & & & & & \\
\hline other & & & & & & & & \\
\hline Subtotal & & & & & & & & \\
\hline ENGINEERS & 10 & 20 & (2) & \%? & 1.20 & $2.2 \%$ & \%थ & 1.2 \\
\hline Chemical & & & & & & & & \\
\hline Civil & & & & & & & & \\
\hline Computer & & & & & & & & \\
\hline Electrical & & & & & & & & \\
\hline Environmental & & & & & & & & \\
\hline Industrial & & & & & & & & \\
\hline Mechanical & & & & & & & & \\
\hline Nuclear & & & & & & & & \\
\hline Petroleum/Mining & & & & & & & & \\
\hline Plant & & & & & & & & \\
\hline Quality Control & & & & & & & & \\
\hline Safety & & & & & & & & \\
\hline Other & & & & & & & & \\
\hline Subtotal & & & & & & & & \\
\hline
\end{tabular}




\begin{tabular}{|c|c|c|c|c|c|c|c|c|}
\hline \multicolumn{4}{|c|}{ Planned Staffing (Full Time Equivalent) } & \multicolumn{5}{|c|}{ Note: Job Family Only After 1997} \\
\hline Job Family: & Number: & $\% \%$ & ४थथ & $\%$ & १२००० & \%. & 1.220 & 2.4 .2 \\
\hline Job Category & & 1996 & 1997 & 1998 & 1999 & 2000 & 2001 & 2002 \\
\hline SCIENTISTS & $3 .+4$ & \%.? & 2. & \%थ। & $1 .+2$ & \%: & 8 & 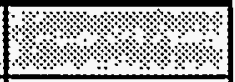 \\
\hline \multicolumn{9}{|l|}{ Chemists } \\
\hline \multicolumn{9}{|l|}{ Environmental } \\
\hline \multicolumn{9}{|l|}{ Geologists } \\
\hline \multicolumn{9}{|l|}{ Life } \\
\hline \multicolumn{9}{|l|}{ Material } \\
\hline \multicolumn{9}{|l|}{ Mathematicians } \\
\hline \multicolumn{9}{|l|}{ Physicists } \\
\hline \multicolumn{9}{|l|}{ Social } \\
\hline \multicolumn{9}{|l|}{ other } \\
\hline \multicolumn{9}{|l|}{ Subtotal } \\
\hline $\begin{array}{l}\text { ADMINISTRATIVE IOTHER } \\
\text { PROFESSIONALS }\end{array}$ & \%? & $1 \%$ & $2 \%$ & $\%$ & \%थ. & 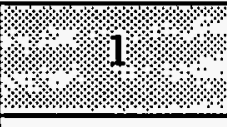 & $1 \%$ & $1 \%$ \\
\hline Accountant/auditor & 1 & 1 & 1 & & & & - & \\
\hline \multicolumn{9}{|l|}{ Architect } \\
\hline \multicolumn{9}{|l|}{ Buyers/Procurement } \\
\hline \multicolumn{9}{|l|}{ Communications } \\
\hline \multicolumn{9}{|l|}{$\begin{array}{l}\text { Compliance } \\
\text { Inspectors }\end{array}$} \\
\hline \multicolumn{9}{|l|}{$\begin{array}{l}\text { Computer Sys } \\
\text { Analyst }\end{array}$} \\
\hline $\begin{array}{l}\text { Cost Est/ planner/ } \\
\text { sch }\end{array}$ & & & & & & & & \\
\hline
\end{tabular}




\begin{tabular}{|c|c|c|c|c|c|c|c|c|}
\hline \multicolumn{4}{|c|}{ Planned Staffing (Full Time Equivalent) } & \multicolumn{5}{|c|}{ Note: Job Family Only After 1997} \\
\hline Job Fami y & Number & ४४४\%, & \%४ & 8 ? & एथ४४थ & ४४४४ & \% & 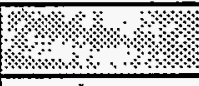 \\
\hline Job Category & & 1996 & 1997 & 1998 & 1999 & 2000 & 2001 & 2002 \\
\hline \multicolumn{9}{|l|}{ Health Physics } \\
\hline \multicolumn{9}{|l|}{ Industrial Hygiene } \\
\hline \multicolumn{9}{|l|}{ Lawyers } \\
\hline \multicolumn{9}{|l|}{$\begin{array}{l}\text { Personnel/labor } \\
\text { Relations }\end{array}$} \\
\hline \multicolumn{9}{|l|}{ Physicians } \\
\hline \multicolumn{9}{|l|}{$\begin{array}{l}\text { Physician } \\
\text { Assistant/Nurse }\end{array}$} \\
\hline \multicolumn{9}{|l|}{$\begin{array}{l}\text { Safeguard \& } \\
\text { Security }\end{array}$} \\
\hline \multicolumn{9}{|l|}{$\begin{array}{l}\text { Tech Writers \& } \\
\text { Editors }\end{array}$} \\
\hline \multicolumn{9}{|l|}{ Trainers } \\
\hline \multicolumn{9}{|l|}{ Other } \\
\hline \multicolumn{9}{|l|}{ Subtotal } \\
\hline $\begin{array}{l}\text { GENERAL } \\
\text { ADMIN/SECRETARY/CLERK }\end{array}$ & \%? & $\% \div \%$ & $1 \%$ & $8.1 \%$ & 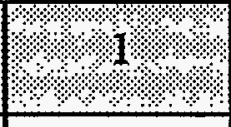 & 1.9 .8 & 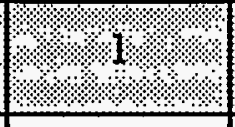 & 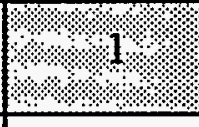 \\
\hline \multicolumn{9}{|l|}{ Admin Assistant } \\
\hline \multicolumn{9}{|l|}{ Office Clerk (Gen) } \\
\hline \multicolumn{9}{|l|}{$\begin{array}{l}\text { Office Clerk } \\
\text { (Special) }\end{array}$} \\
\hline Secretaries & 1 & 1 & 1 & & & & & \\
\hline \multicolumn{9}{|l|}{$\begin{array}{l}\text { Typist/Word } \\
\text { Processor }\end{array}$} \\
\hline Other & & & & & & & & \\
\hline
\end{tabular}




\begin{tabular}{|c|c|c|c|c|c|c|c|c|}
\hline \multicolumn{4}{|c|}{ Planned Staffing (Full Time Equivalent) } & \multicolumn{5}{|c|}{ Note: Job Family Only After 1997} \\
\hline Job Family ${ }^{\prime}$ & Number & अ४०े. & 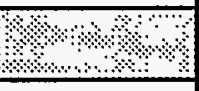 & ध. & $4.8 \%$ & 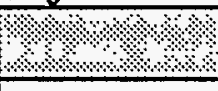 & 1.8 .1 & ४ै।? \\
\hline Job Category & & 1996 & 1997 & 1998 & 1999 & 2000 & 2001 & 2002 \\
\hline \multicolumn{9}{|l|}{ Subtotal } \\
\hline TECHNICIANS & ४ै। & \%, ?. & \%॰ & 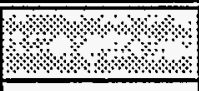 & \%.:. & 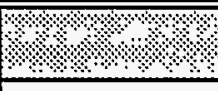 & $1.1 .8 \%$ & $1 \%$ \\
\hline \multicolumn{9}{|l|}{$\begin{array}{l}\text { Computer } \\
\text { Operator/Coder }\end{array}$} \\
\hline \multicolumn{9}{|l|}{ Drafters } \\
\hline \multicolumn{9}{|l|}{ Engineers/Technicians } \\
\hline \multicolumn{9}{|l|}{$\begin{array}{l}\text { Environmental } \\
\text { Science } \\
\text { Technicians }\end{array}$} \\
\hline \multicolumn{9}{|l|}{$\begin{array}{l}\text { Health Physics } \\
\text { Technicians }\end{array}$} \\
\hline \multicolumn{9}{|l|}{$\begin{array}{l}\text { Industrial } \\
\text { Saf/health } \\
\text { Technicians } \\
\end{array}$} \\
\hline \multicolumn{9}{|l|}{$\begin{array}{l}\text { Instru/Control } \\
\text { Technicians }\end{array}$} \\
\hline \multicolumn{9}{|l|}{ Lab. Technicians } \\
\hline \multicolumn{9}{|l|}{ Media Technicians } \\
\hline \multicolumn{9}{|l|}{$\begin{array}{l}\text { Survey/Map } \\
\text { Technicians }\end{array}$} \\
\hline \multicolumn{9}{|l|}{ Other } \\
\hline \multicolumn{9}{|l|}{ Subtotal } \\
\hline GRAFTS & $\% \%$ & \%? \%? & $\%$ & 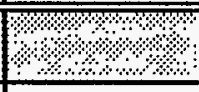 & \% \%. & (... & $1 \% .1 . \% .8$ & $1 \% \% ?$ \\
\hline \multicolumn{9}{|l|}{ Carpenters } \\
\hline Electricians & & & & & & & & \\
\hline
\end{tabular}




\begin{tabular}{|c|c|c|c|c|c|c|c|c|}
\hline \multicolumn{4}{|c|}{ Planned Staffing (Ful1 Time Equivalent) } & \multicolumn{5}{|c|}{ Note: Job Family Only After 1997} \\
\hline Job Famlly. & Number: & 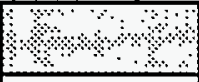 & $1 \%$ & $1 \%$ & ४४ & $2 .+\%$ & ४थ ४ै४ & अथே\%? \\
\hline Job Category & & 1996 & 1997 & 1998 & 1999 & 2000 & 2001 & 2002 \\
\hline \multicolumn{9}{|l|}{ HVAC } \\
\hline \multicolumn{9}{|l|}{ Machinists } \\
\hline \multicolumn{9}{|l|}{ Masons } \\
\hline \multicolumn{9}{|l|}{ Millwrights } \\
\hline \multicolumn{9}{|l|}{ Painters } \\
\hline \multicolumn{9}{|l|}{ Plmbrs/Pipefitters } \\
\hline \multicolumn{9}{|l|}{$\begin{array}{l}\text { Struct/Metal } \\
\text { Workers }\end{array}$} \\
\hline \multicolumn{9}{|l|}{$\begin{array}{l}\text { Vehicl/Mobile } \\
\text { Equip. } \\
\text { Mechanics }\end{array}$} \\
\hline \multicolumn{9}{|l|}{ Welders } \\
\hline \multicolumn{9}{|l|}{ Other } \\
\hline \multicolumn{9}{|l|}{ Subtotal } \\
\hline OPERATORS & थेथ ? & \%थेथे & $1 \% 2 \%$ & \%े ४ \% & $1 \%$ & थः थे? & $1 \% 1 \%$ & \% \% \% \\
\hline \multicolumn{9}{|l|}{ Lt. Vehicle Drivers } \\
\hline \multicolumn{9}{|l|}{$\begin{array}{l}\text { Material Moving } \\
\text { Equip. }\end{array}$} \\
\hline \multicolumn{9}{|l|}{ Nuclear Plant } \\
\hline \multicolumn{9}{|l|}{$\begin{array}{l}\text { Utilities Waste } \\
\text { Proces }\end{array}$} \\
\hline \multicolumn{9}{|l|}{ Other } \\
\hline Subtotal & & & & & & & & \\
\hline
\end{tabular}




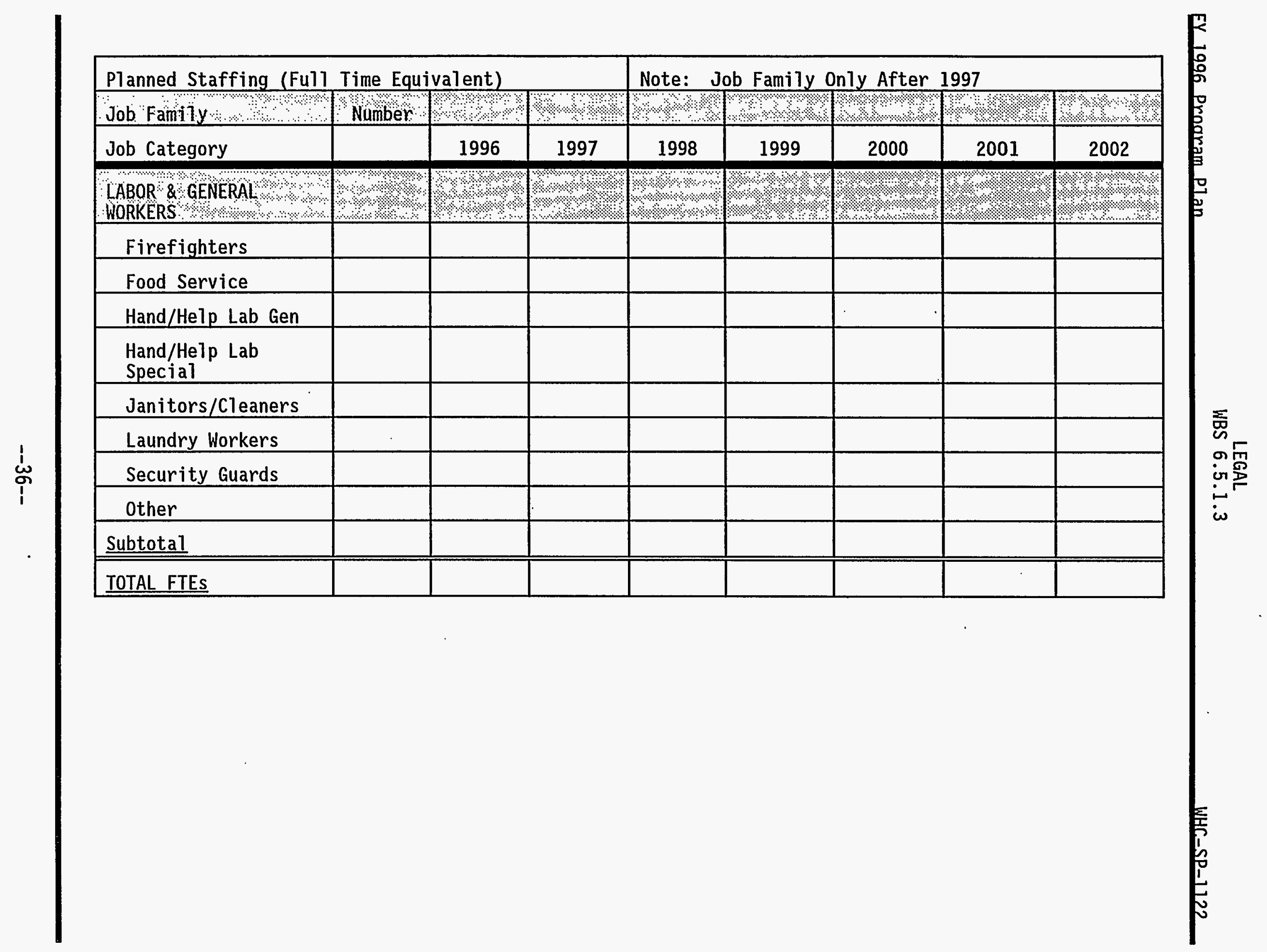




\subsubsection{Human Resources (WBS 6.5.1.4)}

\section{$\underline{\text { Mission }}$}

The mission of the Human Resources Department is to manage Recruitment and Placement, Diversity, Employee Concerns and Compensation programs.

\section{Product/Services Provided}

The primary services performed by the Human Resources Department are: Maintain staffing level in accordance with WHC/RL direction through recruitment and out-placement of personnel, ensure compliance with EEO regulations, produce affirmative action plan, administer merit and promotion increases, and maintain an Employee Concerns Program.

\section{Primary Customer}

Primary customers of Human Resources. Department include: ICF Kaiser Hanford, U.S. Department of Energy (RL \& HQ), Westinghouse Hanford Company, Boeing Computer Services, Pacific Northwest Laboratory, Hanford Environmental Health Foundation and Bechtel Hanford Incorporated. 


\begin{tabular}{|c|c|c|c|c|c|c|c|c|}
\hline $\begin{array}{l}\text { Work } \\
\text { Breakdown } \\
\text { Structure } \\
\text { Dictionary }\end{array}$ & \multicolumn{5}{|c|}{$\begin{array}{l}\text { Westinghouse Hanford Company } \\
\text { HUMAN RESOURCES } \\
\text { Part 1 - Summary } \\
\text { (Dollars in } 000^{\prime} s \text { ) }\end{array}$} & \multicolumn{3}{|c|}{$\begin{array}{l}\text { FY } 1996 \\
\text { Indirect } \\
\text { Program Plan } \\
\text { Rev. \# } 0 \\
\text { 15-Sep-95 }\end{array}$} \\
\hline $\begin{array}{c}\text { Cost Account Number } \\
\text { IMKDAG }\end{array}$ & \multicolumn{5}{|l|}{$\begin{array}{l}\text { Cost Account Tille } \\
\text { HUMAN RESOURCES }\end{array}$} & \multicolumn{3}{|c|}{$\begin{array}{l}\text { Proposed Rate: } \\
\text { (Rated Service Pool Only) }\end{array}$} \\
\hline $\begin{array}{r}\text { SMS WBS Number } \\
6.5 .1 .4 \\
\end{array}$ & \multicolumn{5}{|l|}{$\begin{array}{l}\text { SMS Title } \\
\text { HUMAN RESOURCES }\end{array}$} & \multicolumn{3}{|c|}{$\begin{array}{l}\text { Funding Source: } \\
x\end{array}$} \\
\hline RL SMS Program Manger & & & & & & \multicolumn{3}{|l|}{ sws } \\
\hline Cost Account Manager & \multicolumn{5}{|l|}{ VC HODGIN } & \multicolumn{3}{|l|}{ OST } \\
\hline WHC SMS Program Manager & & & & & & \multicolumn{3}{|l|}{$\mathrm{DOH}$} \\
\hline Financial Manager & \multicolumn{5}{|l|}{ MADAVIS } & \multicolumn{3}{|l|}{ MGT PRO } \\
\hline Responsible Analyst & \multicolumn{5}{|l|}{ ML VAN LIEW } & \multirow{2}{*}{\multicolumn{3}{|c|}{\begin{tabular}{|l} 
POOL \\
FY 1996 Baseline \\
Full-Time Equivalents (FTEs)
\end{tabular}}} \\
\hline & \multicolumn{2}{|c|}{$\begin{array}{c}\text { FY } 1996 \text { Approved Funding } \\
\text { Full-Time Equivalents (FTEs) }\end{array}$} & \multicolumn{3}{|c|}{$\begin{array}{l}\text { FY } 1996 \text { Unfunded } \\
\text { Full-Time Equivalents (FTEs) }\end{array}$} & & & \\
\hline TYPE OF FTE & \begin{tabular}{l|l|} 
Exempt & Non-Exempt \\
\end{tabular} & Bargaining & Exempt & Non-Exempt & Bargaining & Exempt & Non-Exempt & Bargaining \\
\hline Organizational & 7 & 3 & & & & 7 & 1 & 3 \\
\hline Support & 0.1 & & & & & 0.1 & 0 & 0 \\
\hline TOTALFTES & 7.1 & 3 & & 0 & 0 & 7.1 & 1 & 3 \\
\hline COST ELEMENTS & FY 1996 Approved Fundi & ing Budget & FY $1:$ & 996 Unfunded Bi & udget & FY 1: & 1996 Baseline B & Budget \\
\hline . Labor - Regular & 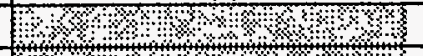 & 651.7 & $88,1,1$ & 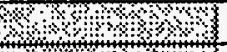 & & $184,8 \%$ & 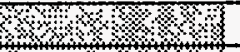 & 651.7 \\
\hline . Labor - Overtime & $18 \times 3+13$ & 0 & 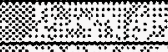 & $3,1 \% 43$ & & 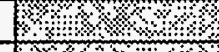 & $4 \% \% \%$ & 0 \\
\hline 0 Total Labor & 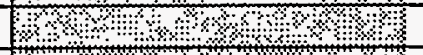 & 651.7 & 40 & $18,6,1$ & 0 & 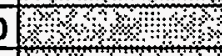 & 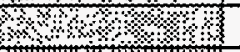 & 651.7 \\
\hline 1 Materials & $14 \%, 013 \%$ & 11.8 & $4 \%$ & $2 \% 4 \% 4$ & & $1 \times 1 \%$ & $8<2 \% \% \%$ & 11.8 \\
\hline 2 Purchased Services & 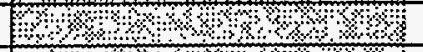 & 55.6 & 18 & 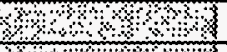 & & $10 \% 3$ & $40 \% 1 \%$ & 55.6 \\
\hline 3 Other Hanford & 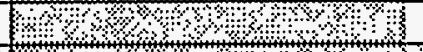 & 0 & $4,16 \%$ & 406, & & $18+8 \times 14 \%$ & 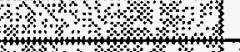 & 0 \\
\hline Subtotal Originated Costs & $100 \% 1 \%$ & $7 \times 7191$ & $4 \%, \%$ & $84 \% \div$ & आ००० & 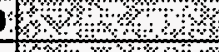 & $40 \times 3 \%$ & $7.19: 1$ \\
\hline 4 Site Services & $14+4 \mathrm{P}+4 \%$ & 7.8 & 1\%. & ४⿻ +1\% & & W४\$ & $3 \times 1,4$ & 7.8 \\
\hline 5 Internal Charges & $1040 \%, 1,0101 \%$ & 0 & $1 \%, 13$ & 36,4 & & $3 \times 1,30,4$ & 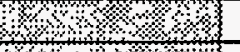 & 0 \\
\hline 6 IRM Support & 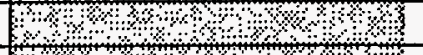 & & $4+4$ & $43,1 \%$ & & $1 \%$ & $4,6,3,13$ & 2 \\
\hline 7 Overheads & 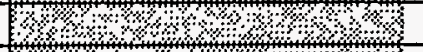 & & $14,4,14$ & $146, \%$ & & $344 \div \div$ & $140,14,4$ & 0 \\
\hline 8 Revenue & 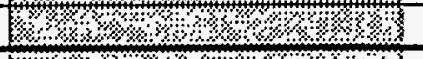 & & $30 \%$ & $43 \%$ & & $2 \%$ & 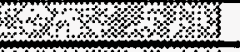 & 0 \\
\hline TOTAL DOLLARS & 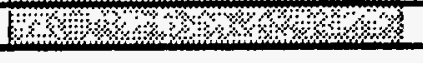 & 728.9 & \% & \$४४ \% \% & & 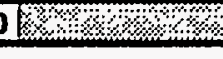 & \%४\% \% & 728.9 \\
\hline $\begin{array}{l}\text { SIGNATURES } \\
\text { Financial Analyst: MnQ } \\
\text { CAM: }\end{array}$ & Vandiein & Date: $9 / 15 / 95$ & & BASELINE APP & PROVAL & & & Date: \\
\hline
\end{tabular}


HUMAN RESOURCES DIVISION

\section{C. HODGIN}

\begin{tabular}{|c|c|c|c|c|}
\hline \multicolumn{5}{|l|}{ BUILDING BLOCKS FOR FY 1996} \\
\hline \multicolumn{5}{|l|}{ FY 1996 Building Blocks (5000) } \\
\hline $\begin{array}{l}\text { ORGANIZATIONAL MORK SCOPE DESCRIPTIOH } \\
\text { (b-blocks.rEV - THKDAG) }\end{array}$ & $\begin{array}{l}\text { Org. FTES } \\
\text { in } \\
\text { Workscope }\end{array}$ & $\begin{array}{l}\text { Support } \\
\text { FTEs in } \\
\text { Horkscope }\end{array}$ & $\begin{array}{c}\text { FY } 1996 \\
\text { Originated } \\
\text { Cost" }\end{array}$ & $\begin{array}{l}\text { JUSTIFICATION OF SCOPE/IMPACT } \\
\text { IF HOT FULDED }\end{array}$ \\
\hline $\begin{array}{l}\text { Human Resources Manager and Executive Secretary, } \\
\text { Manage Recruitment and Pl acement, Diversity. } \\
\text { Employee Concerns and Compensation } \\
\text { Support from Others for Computer Tech assistance. }\end{array}$ & 2 & .1 & $\begin{array}{r}107.1 K \\
7.4\end{array}$ & $\begin{array}{l}\text { ICF KH would have no centralized financial } \\
\text { management. This would result in disjointed HR } \\
\text { Policy and increased risk. }\end{array}$ \\
\hline Compensation & 1.25 & 0 & 81.5 & $\begin{array}{l}\text { ICF KH would not maintain a compensation program } \\
\text { which adninisters merit and promotion increases } \\
\text { and ensures equity in pay. }\end{array}$ \\
\hline $\begin{array}{l}\text { Diversity Progran } \\
\text {. }\end{array}$ & 1 & $\overline{0}$ & 42.4 & $\begin{array}{l}\text { ICF would not mointain a Diversity office, } \\
\text { produce an Affirmative Action Plan or respond to } \\
\text { Equal Employment Opportunity complaints and/or } \\
\text { charges. }\end{array}$ \\
\hline Enployee Concerns Program & 1.25 & 0 & 81.5 & $\begin{array}{l}\text { ICF KH would not maintain an Employee concerns } \\
\text { Program }\end{array}$ \\
\hline LEVEL 1 TOTALS & & & & \\
\hline
\end{tabular}

* Originated Cost $=$ Cost elements $0,1,2,3$ 
HUMAN RESOURCES DIVISION

V. C. HODGIN

\begin{tabular}{|c|c|c|c|c|}
\hline BUILDING BLOCKS FOR FY 1996 & & & & \\
\hline FY 1996 Building Blocks (s000) & & & & \\
\hline ORGANIZATIOHAL HORK SCOPE DESCRIPTIOH & $\begin{array}{l}\text { Org in FTES } \\
\text { Morkscope }\end{array}$ & $\begin{array}{l}\text { Support } \\
\text { FTES in } \\
\text { Horkscope }\end{array}$ & $\begin{array}{c}\text { FY } 1996 \\
\text { Originated } \\
\text { Cost* }\end{array}$ & $\begin{array}{l}\text { JUSTIFICATION OF SCOPE/IHPACT } \\
\text { IF NOT FUNDED }\end{array}$ \\
\hline $\begin{array}{l}\text { Recruit tment and Placement (Hire, Terminate, Maintain } \\
\text { Staff) }\end{array}$ & 4.75 & 0 & 276.8 & \\
\hline Enhance Company Image & .75 & 0 & 55.1 & 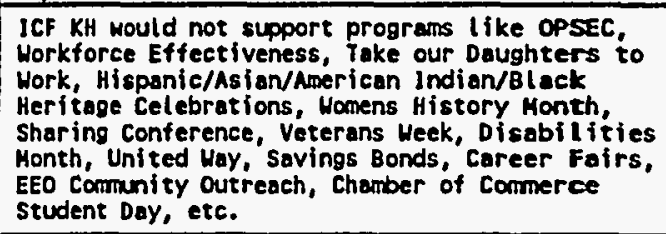 \\
\hline Other Cost Elements included: $(1,2,3)$ & & & 67.3 & \\
\hline 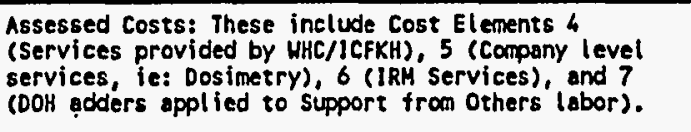 & & & 9.8 & \\
\hline LEVEL' 9 TOTALS & 11 & & $\begin{array}{l}719.1 \\
728.8 \\
72.9\end{array}$ & $\begin{array}{l}\text { Met Originated cost } \\
\text { Assersed cost } \\
\text { Totol Budget }\end{array}$ \\
\hline
\end{tabular}

* Originated Cost $=$ Cost elements $0,1,2,3$ 


\begin{tabular}{|c|c|c|c|c|c|c|c|c|}
\hline \multicolumn{4}{|c|}{ P7anned Staffing (Ful1 Time Equivalent) } & \multicolumn{5}{|c|}{ Note: Job Family Only After 1997} \\
\hline Job Family & Number. & 20 & 20 & +2 & 1: & 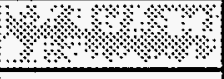 & 20 & 2.2. \\
\hline Job Category & & 1996 & 1997 & 1998 & 1999 & 2000 & 2001 & 2002 \\
\hline MANAGERS & 2 & $(-2)$ & थ. & 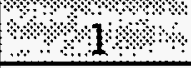 & 1.1 & $4.1 \%$ & (- 1 & $+\infty$ \\
\hline \multicolumn{9}{|l|}{ First line } \\
\hline General/executive & 1 & 1 & 1 & & & & & \\
\hline \multicolumn{9}{|l|}{ Projec/Program } \\
\hline \multicolumn{9}{|l|}{ other } \\
\hline \multicolumn{9}{|l|}{ Subtotal } \\
\hline ENGINEERS & 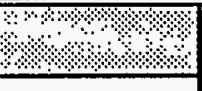 & 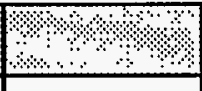 & 1.2 .2 & 1.2 .2 & 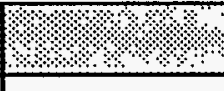 & 1थ? & 1.12 & एथ०० \\
\hline \multicolumn{9}{|l|}{ Chemical } \\
\hline \multicolumn{9}{|l|}{ Civil } \\
\hline \multicolumn{9}{|l|}{ Computer } \\
\hline \multicolumn{9}{|l|}{ Electrical } \\
\hline \multicolumn{9}{|l|}{ Environmental } \\
\hline \multicolumn{9}{|l|}{ Industrial } \\
\hline \multicolumn{9}{|l|}{ Mechanical } \\
\hline \multicolumn{9}{|l|}{ Nuclear } \\
\hline \multicolumn{9}{|l|}{ Petroleum/Mining } \\
\hline \multicolumn{9}{|l|}{ Plant } \\
\hline \multicolumn{9}{|l|}{ Quality Control } \\
\hline \multicolumn{9}{|l|}{ Safety } \\
\hline \multicolumn{9}{|l|}{ Other } \\
\hline Subtotal & & & & & & & & \\
\hline
\end{tabular}




\begin{tabular}{|c|c|c|c|c|c|c|c|c|}
\hline \multicolumn{4}{|c|}{ Planned Staffing (Full Time Equivalent) } & \multicolumn{5}{|c|}{ Note: Job Family Only After 1997} \\
\hline Job Famtiy \% ? & Number & $8 \%$ & 12.2 & 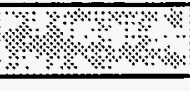 & $2.2 \%$ & ?.2\% & एथथ & $\%$ \\
\hline Job Category & & 1996 & 1997 & 1998 & 1999 & 2000 & 2001 & 2002 \\
\hline SCIENTISTS & 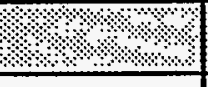 & $1 \%$ & ?. & $1.4 ., \%$ & 2.1 .2 & 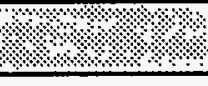 & (2.2. & $1 \%$ \\
\hline Chemists & & & & & & & & \\
\hline Environmental & & & & & & & & \\
\hline Geologists & & & & & & & & \\
\hline Life & & & & & & & & \\
\hline Material & & & & & & & & \\
\hline Mathematicians & & & & & & & & \\
\hline Physicists & & & & & & & & \\
\hline Social & & & & & & & & \\
\hline Other & & & & & & & & \\
\hline Subtotal & & & & & & & & \\
\hline $\begin{array}{l}\text { ADMINISTRATIVE } 10 T H E R \\
\text { PROFESSIONALS }\end{array}$ & $8.8 \%$ & $1 \%$ & 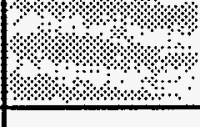 & $18 \%$ & (6?:? & $1.6 \%$ & 1.6 & 18.6 \\
\hline Accountant/auditor & & & & & & & . & \\
\hline Architect & & & & & & & & \\
\hline Buyers/Procurement & & & & & & & & \\
\hline Communications & & & & & & & & \\
\hline $\begin{array}{l}\text { Compliance } \\
\text { Inspectors }\end{array}$ & & & & & & & & \\
\hline $\begin{array}{l}\text { Computer Sys } \\
\text { Analyst }\end{array}$ & & & & & & & & \\
\hline $\begin{array}{l}\text { Cost Est/planner/ } \\
\text { sch }\end{array}$ & & & & & & & & \\
\hline
\end{tabular}




\begin{tabular}{|c|c|c|c|c|c|c|c|c|}
\hline \multicolumn{4}{|c|}{ Planned Staffing (Ful1 Time Equivalent) } & \multicolumn{5}{|c|}{ Note: Job Family Only After 1997} \\
\hline Job Famfiy & Number: & ४० & . & स\% & 17.2. & थथ & 4 & 10.20 \\
\hline Job Category & & 1996 & 1997 & 1998 & 1999 & 2000 & 2001 & 2002 \\
\hline \multicolumn{9}{|l|}{ Health Physics } \\
\hline \multicolumn{9}{|l|}{ Industrial Hygiene } \\
\hline \multicolumn{9}{|l|}{ Lawyers } \\
\hline $\begin{array}{l}\text { Personnel/labor } \\
\text { Relations }\end{array}$ & 6 & 6 & 6 & & & & & \\
\hline \multicolumn{9}{|l|}{ Physicians } \\
\hline \multicolumn{9}{|l|}{$\begin{array}{l}\text { Physician } \\
\text { Assistant/Nurse }\end{array}$} \\
\hline \multicolumn{9}{|l|}{$\begin{array}{l}\text { Safeguard \& } \\
\text { Security }\end{array}$} \\
\hline \multicolumn{9}{|l|}{$\begin{array}{l}\text { Tech Writers \& } \\
\text { Editors }\end{array}$} \\
\hline \multicolumn{9}{|l|}{ Trainers } \\
\hline \multicolumn{9}{|l|}{ other } \\
\hline \multicolumn{9}{|l|}{ Subtotal } \\
\hline $\begin{array}{l}\text { GENERAL } \\
\text { ADMIN/SECRETARY/CLLERK }\end{array}$ & 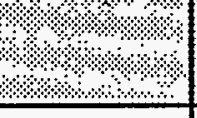 & $\sqrt{2}$ & 2. & 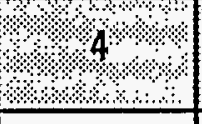 & 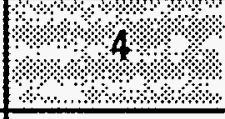 & 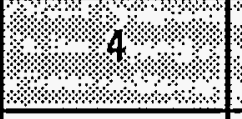 & 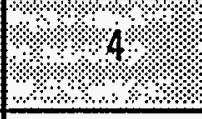 & 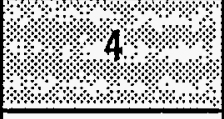 \\
\hline \multicolumn{9}{|l|}{ Admin Assistant } \\
\hline Office Clerk (Gen) & 3 & 3 & 3 & & & & & \\
\hline \multicolumn{9}{|l|}{$\begin{array}{l}\text { Office Clerk } \\
\text { (Special) }\end{array}$} \\
\hline Secretaries & 1 & 1 & 1 & & & & & \\
\hline \multicolumn{9}{|l|}{$\begin{array}{l}\text { Typist/Word } \\
\text { Processor }\end{array}$} \\
\hline Other & & & & & & & & \\
\hline
\end{tabular}




\begin{tabular}{|c|c|c|c|c|c|c|c|c|}
\hline \multicolumn{4}{|c|}{ Planned Staffing (Full Time Equivalent) } & \multicolumn{5}{|c|}{ Note: Job Family Only After 1997} \\
\hline Job Family $\%$ ? & Number & \%॰ & थे० & \%०० & 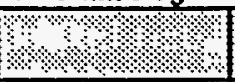 & $2.2 \%$ & \% ४ \% \% & \%थे? \\
\hline Job Category & & 1996 & 1997 & 1998 & 1999 & 2000 & 2001 & 2002 \\
\hline \multicolumn{9}{|l|}{ Subtotal } \\
\hline TECHNICIANS & ४.\%? & अै? & \%..? & \%ै। & 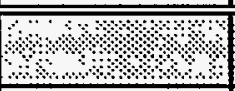 & $1 \% ? \%$ & (4) & \% \% \% \% \\
\hline \multicolumn{9}{|l|}{$\begin{array}{l}\text { Computer } \\
\text { Operator/Coder }\end{array}$} \\
\hline \multicolumn{9}{|l|}{ Drafters } \\
\hline \multicolumn{9}{|l|}{ Engineers/Technicians } \\
\hline \multicolumn{9}{|l|}{$\begin{array}{l}\text { Environmental } \\
\text { Science } \\
\text { Technicians }\end{array}$} \\
\hline \multicolumn{9}{|l|}{$\begin{array}{l}\text { Health Physics } \\
\text { Technicians }\end{array}$} \\
\hline \multicolumn{9}{|l|}{$\begin{array}{l}\text { Industrial } \\
\text { Saf/health } \\
\text { Technicians }\end{array}$} \\
\hline \multicolumn{9}{|l|}{$\begin{array}{l}\text { Instru/Contro] } \\
\text { Technicians }\end{array}$} \\
\hline \multicolumn{9}{|l|}{ Lab. Technicians } \\
\hline \multicolumn{9}{|l|}{ Media Technicians } \\
\hline \multicolumn{9}{|l|}{$\begin{array}{l}\text { Survey/Map } \\
\text { Technicians }\end{array}$} \\
\hline \multicolumn{9}{|l|}{ Other } \\
\hline \multicolumn{9}{|l|}{ Subtotal } \\
\hline CRAFTS. & $1 \%$ \% & \%?\% & $1 \% 2$ & $1 \%$ & \%: : & $12.1 \%$ & \%? & 19.2\%? \\
\hline \multicolumn{9}{|l|}{ Carpenters } \\
\hline Electricians & & & & & & & & \\
\hline
\end{tabular}




\begin{tabular}{|c|c|c|c|c|c|c|c|c|}
\hline \multicolumn{4}{|c|}{ Planned Staffing (Ful1 Time Equivalent) } & \multicolumn{5}{|c|}{ Note: Job Family Only After 1997} \\
\hline Job Family \% & Number & 28 & $1 \%$ & \%० & $1.8 \%$ & $2.2 \%$ & 1.2 .19 & 18. \\
\hline Job Category & & 1996 & 1997 & 1998 & 1999 & 2000 & 2001 & 2002 \\
\hline \multicolumn{9}{|l|}{ HVAC } \\
\hline \multicolumn{9}{|l|}{ Machinists } \\
\hline Masons & & & & & & $\dot{-}$ & & . \\
\hline \multicolumn{9}{|l|}{ Millwrights } \\
\hline \multicolumn{9}{|l|}{ Painters } \\
\hline \multicolumn{9}{|l|}{ Plmbrs/Pipefitters } \\
\hline \multicolumn{9}{|l|}{$\begin{array}{l}\text { Struct/Metal } \\
\text { Workers }\end{array}$} \\
\hline \multicolumn{9}{|l|}{$\begin{array}{l}\text { Vehicl/Mobile } \\
\text { Equip. } \\
\text { Mechanics }\end{array}$} \\
\hline \multicolumn{9}{|l|}{ Welders } \\
\hline \multicolumn{9}{|l|}{ other } \\
\hline \multicolumn{9}{|l|}{ Subtotal } \\
\hline OPERATORS & \%.\%? & \%े. & \%. & $\%$ & 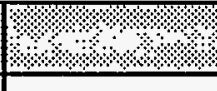 & $1 \%$ & $1 \% . \%$ & $1 \%$ \\
\hline \multicolumn{9}{|l|}{ Lt. Vehicle Drivers } \\
\hline \multicolumn{9}{|l|}{$\begin{array}{l}\text { Material Moving } \\
\text { Equip. }\end{array}$} \\
\hline \multicolumn{9}{|l|}{ Nuclear Plant } \\
\hline \multicolumn{9}{|l|}{$\begin{array}{l}\text { Utilities Waste } \\
\text { Proces }\end{array}$} \\
\hline \multicolumn{9}{|l|}{ Other } \\
\hline Subtotal & & & & & & & & \\
\hline
\end{tabular}




\begin{tabular}{|c|c|c|c|c|c|c|c|c|}
\hline \multicolumn{4}{|c|}{ Planned Staffing (Ful1 Time Equivalent) } & \multicolumn{5}{|c|}{ Note: Job Family Only After 1997} \\
\hline Job Family $\mathrm{al}$ & Number & अ? & अ० & केष & ४৮ ২ & 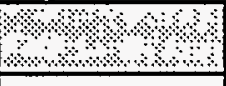 & ४\% \% & $1 \%, \%$ \\
\hline Job Category & & 1996 & 1997 & 1998 & 1999 & 2000 & 2001 & 2002 \\
\hline $\begin{array}{l}\text { LABOR } \& \text { GENERAL } \\
\text { WORKERS }\end{array}$ & \%००.? & \%ै? & \%ै। & $2+\%$ & \% & $1.2 .8 \%$ & ? & +2 \\
\hline \multicolumn{9}{|l|}{ Firefighters } \\
\hline \multicolumn{9}{|l|}{ Food Service } \\
\hline \multicolumn{9}{|l|}{ Hand/Help Lab Gen } \\
\hline \multicolumn{9}{|l|}{$\begin{array}{l}\text { Hand/Help Lab } \\
\text { Special }\end{array}$} \\
\hline \multicolumn{9}{|l|}{ Janitors/Cleaners } \\
\hline \multicolumn{9}{|l|}{ Laundry Workers } \\
\hline \multicolumn{9}{|l|}{ Security Guards } \\
\hline \multicolumn{9}{|l|}{ Other } \\
\hline \multicolumn{9}{|l|}{ Subtotal } \\
\hline TOTAL FTES & & & & & & & & \\
\hline
\end{tabular}


3.1.5 Organizational Development and Total Quality Management (WBS 6.5.1.5)

\section{$\underline{\text { Mission }}$}

The mission of the Organizational Development and Total Quality Management Department is to provide support and guidance throughout the company, to all employees, teams and organizations, in the development and contract performance of the ICF Kaiser Hanford Company.

\section{Products/Services Provided}

The Organizational Development and Total Quality Management organization performs the following:

- Manage a company work Performance Management Program which includes setting roll-down measurement systems that indicate value added success indicators, an award fee process, a cost savings tracking and recognition program, and a PBI oversight, guidance and administration function.

- $\quad$ Provide support on an ad hoc basis and contract compliance activities that assure correct charging of corporate activities and assure the accuracy and integrity of award fee and PBI data.

- $\quad$ Manage the development and systematic oversight of company and Hanford-wide strategies. This includes make/buy directions and actions, economic transition activities and communication with labor, etc. on these initiatives, and other company improvement initiatives.

- $\quad$ Provide the resources to guide employee and management professional development, including mentoring and diversity enhancement programs.

\section{Primary Customers}

Primary customers of the Organizational Development and Total Quality Management Department are: ICF Kaiser Hanford, Westinghouse Hanford Company, and U.S. Department of Energy (RL). 


\begin{tabular}{|c|c|c|c|c|c|c|c|c|}
\hline $\begin{array}{l}\text { Work } \\
\text { Breakdown } \\
\text { Structure } \\
\text { Dictionary }\end{array}$ & \multicolumn{5}{|c|}{$\begin{array}{c}\text { Westinghouse Hanford Company } \\
\text { ORGANIZATIONAL DEVELOPMENT AND TOTAL QUALTY MANAGEMENT } \\
\text { Part I - Summary } \\
\text { (Dollars in } 000^{\prime} \text { 's) }\end{array}$} & \multicolumn{3}{|c|}{$\begin{array}{l}\text { FY } 1996 \\
\text { Indirect } \\
\text { Program Plan } \\
\text { Rev. \# } 0 \\
15-\text { Sep-95 }\end{array}$} \\
\hline $\begin{array}{c}\text { Cost Account Number } \\
\text { IMKDAC }\end{array}$ & \multicolumn{5}{|c|}{$\begin{array}{l}\text { Cost Account Title } \\
\text { ORGANIZATIONAL DEVELOPMENT AND TOTAL QUALTY MANAGEMENT }\end{array}$} & \multicolumn{3}{|c|}{$\begin{array}{l}\text { Proposed Rate: } \\
\text { (Rated Service Pool Only) }\end{array}$} \\
\hline $\begin{array}{r}\text { SMS WBS Number } \\
6.5 .1 .5 \\
\end{array}$ & \multicolumn{5}{|c|}{$\begin{array}{l}\text { SMS Title } \\
\text { ORGANIZATIONAL DEVELOPMENT AND TOTAL QUALTY MANAGEMENT }\end{array}$} & \multicolumn{3}{|c|}{ G\&A $\quad \mathrm{X} \quad$ Funding Source: } \\
\hline RL SMS Program Manger & & & & & & \\
\hline Cost Account Manager & \multicolumn{5}{|l|}{ WA KITCHEN } & \multicolumn{3}{|l|}{ OST } \\
\hline WHC SMS Program Manager & & \multirow{2}{*}{\multicolumn{3}{|c|}{\begin{tabular}{|l|} 
DOH \\
MGT PRO
\end{tabular}}} \\
\hline Financial Manager & \multicolumn{5}{|l|}{ MADAVIS } & & & \\
\hline Responsible Analyst & \multicolumn{5}{|l|}{ ML VAN LIEW } & \multicolumn{3}{|l|}{ POOL } \\
\hline & \multicolumn{2}{|c|}{$\begin{array}{l}\text { FY } 1996 \text { Approved Funding } \\
\text { Full-Time Equivalents (FTEs) }\end{array}$} & \multicolumn{3}{|c|}{$\begin{array}{l}\text { FY } 1996 \text { Unfunded } \\
\text { Full-Time Equivalents (FTEs) }\end{array}$} & \multicolumn{3}{|c|}{$\begin{array}{l}\text { FY } 1996 \text { Baseline } \\
\text { Full-Time Equivalents (FTEs) }\end{array}$} \\
\hline TYPE OF FTE & Exempt Non-Exempt & Bargaining & Exempt & Non-Exempt & Bargaining & Exempt If & Non-Exempt & Bargaining \\
\hline Organizational & 10 & & & & & 10 & 1 & 0 \\
\hline Support & & & & & & 0 & 0 & $\overline{0}$ \\
\hline TOTALFTES & 10 & 01 & 1 & 0 & & 10 & 11 & 0 \\
\hline COST ELEMENTS & FY 1996 Approved Fundi & ding Budget & FY 1 & 996 Unfunded $B$ & udget & FY 1s & 1996 Baseline B & Budget \\
\hline . Labor - Regular & ( & 548.2 & . & 标 & & 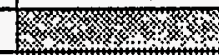 & 勧 & 548.2 \\
\hline . Labor - Overtime & 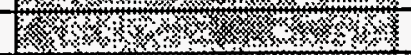 & & 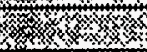 & $x_{1}$ & & 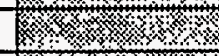 & 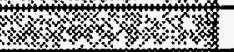 & 0 \\
\hline 0 Total Labor & 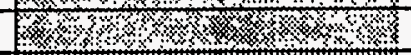 & 548.2 & 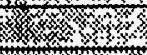 & 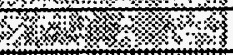 & & 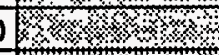 & (\%) & 548.2 \\
\hline 1 Materials & 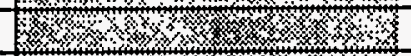 & 3.3 & 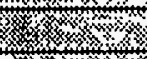 & 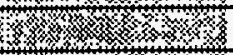 & & $x_{x}$ & 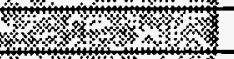 & 3.3 \\
\hline 2 Purchased Senvices & 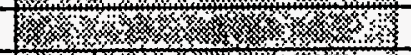 & 50.3 & 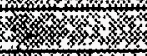 & $x_{x}$ & & \% & $(8,8,0$ & 50.3 \\
\hline 3 Other Hanford & $y_{2}$ & & 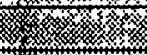 & 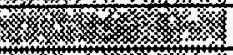 & & 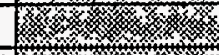 & 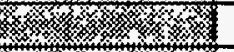 & 0 \\
\hline Subtotal Originated Costs & 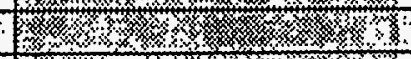 & $\because 601: 8$ & 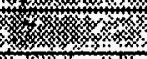 & 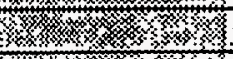 & $\therefore$ & i & 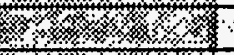 & $\therefore \ldots 601: 8$ \\
\hline 4 Site Senvices & 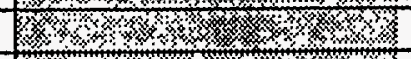 & 4.5 & \% & K. & & 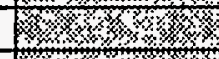 & \% & 4.5 \\
\hline 5 Internal Charges & 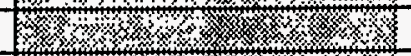 & & $<x$ & $8 x, x_{2}$ & & 8 1... & 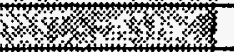 & 0 \\
\hline 6 IRM Support & 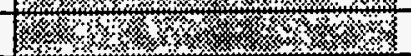 & & < & $x_{x}$ & & $x$ & (3) & 3 \\
\hline 7 Overheads & 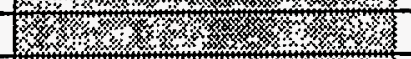 & & 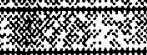 & 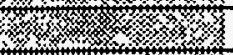 & & 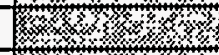 & 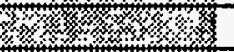 & 0 \\
\hline 8 Revenue & X & & 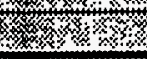 & 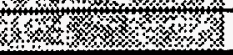 & & 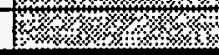 & 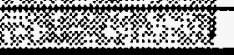 & 0 \\
\hline TOTAL DOLLAAS & 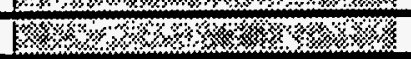 & 609.3 & 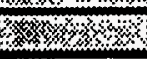 & Z7s & & 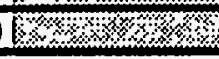 & 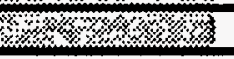 & 609.3 \\
\hline \multicolumn{9}{|c|}{ *Budget does not include the cost of direct labor. ( $\$ 220.5 \mathrm{~K})$} \\
\hline $\begin{array}{l}\text { SIGNATURES } \\
\text { Financia Analyst: ZM T } \\
\text { CAM: }\end{array}$ & Vanckewi & Date: $9 / 15 / 25$ & & BASELINE APP & ROVAL & & & Date: \\
\hline
\end{tabular}


ORGANIZATION DEVELOPMENT AND TOTAL QUALITY MANAGEMENT

\begin{tabular}{|c|c|c|c|c|}
\hline \multicolumn{5}{|l|}{ BUILDING BLOCKS FOR FY 1996} \\
\hline \multicolumn{5}{|l|}{ FY 1996 Building Blocks (S000) } \\
\hline $\begin{array}{l}\text { ORGANIZATIOHAL WORK SCOPE DESCRIPTIOH } \\
\text { (ATTACH3 - IMKDAC) }\end{array}$ & $\begin{array}{l}\text { Org. FTEs } \\
\text { in } \\
\text { Norkscope }\end{array}$ & $\begin{array}{l}\text { Support } \\
\text { FTEs in } \\
\text { Horkscope }\end{array}$ & $\begin{array}{c}\text { FY } 1996 \\
\text { Originated } \\
\text { Cost* }\end{array}$ & $\begin{array}{l}\text { JUSTIFICATIOH OF SCOPE/IMPACT } \\
\text { IF NOT FUNDED }\end{array}$ \\
\hline $\begin{array}{l}\text { The Company Performance Hanagement progran includes } \\
\text { setting roll-down measurement systems which indicate } \\
\text { value added success indicators, award fee. processes, } \\
\text { cost savings tracking and recognition, and PBI } \\
\text { actninistration. }\end{array}$ & 3.0 & See Note & $\$ 220.5 \mathrm{~K}$ & $\begin{array}{l}\text { All activities in this category are essential to } \\
\text { the effective management of the company and } \\
\text { operations within a performance based } \\
\text { enviroment. Reductions in this area will } \\
\text { adversely effect our ability to meet our } \\
\text { contractual agreements relative to performance. }\end{array}$ \\
\hline $\begin{array}{l}\text { Providing Executive support on an ad hoc basis and } \\
\text { contract compl iance activities that assure correct } \\
\text { charging of corporate activities and assuring the } \\
\text { aceuracy and integrity of award fee and PBI data. }\end{array}$ & 2.5 & Ses Note & $\$ 183.8 \mathrm{~K}$ & $\begin{array}{l}\text { This organization is called on frequently to } \\
\text { support the executives in developing the } \\
\text { information to fulfill the requests. Also, as } \\
\text { pBls are reported as complete, independent } \\
\text { varification of data and assurance that submittal } \\
\text { meets all criteria is provided by this org. } \\
\text { Reduction or el imination of this service could } \\
\text { jeopardize the quality and integrity of } \\
\text { information supplied to our customer and client. }\end{array}$ \\
\hline $\begin{array}{l}\text { Managing the deployment and systematic oversight of } \\
\text { company and Hanford-wide strategies. This includes } \\
\text { make/buy directions and actions, economic transition } \\
\text { activities and commications with labor, ete. on } \\
\text { these initiatives, and other company improvement } \\
\text { initiatives. }\end{array}$ & 1.8 & See Hote & $592.4 \mathrm{~K}$ & $\begin{array}{l}\text { This function is essential in maintaining focus } \\
\text { and accountability in pursuit of the various } \\
\text { strategies at the company and Hanford site level, } \\
\text { especially during these times of transition and } \\
\text { down sizing. }\end{array}$ \\
\hline 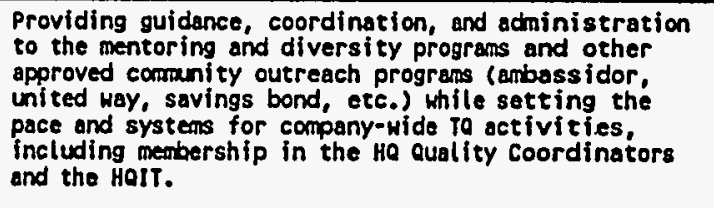 & .7 & See Note & $\$ 51.5 \mathrm{~K}$ & $\begin{array}{l}\text { Reduction or el imination of this function would } \\
\text { impact our course of action to establish and } \\
\text { maintain a basel ine (Malcom Baldrige), infuse a } \\
\text { to culture, and support approved comminity } \\
\text { partnering activities. }\end{array}$ \\
\hline Direct Support & 3 & & $(\$ 220.5)$ & $\begin{array}{l}\text { Cost of Direct Labor Support, not part of Met } \\
\text { Originated Cost. }\end{array}$ \\
\hline Other Costs included: $(1,2,3)$ & & & $553.6 \mathrm{~K}$ & \\
\hline $\begin{array}{l}\text { Assessed Costs: These include Cost Elements } 4 \\
\text { (Services provided by HHC/ICFKH), } 5 \text { (Company level } \\
\text { services, ie. Dosimetry), } 6 \text { (IRH Services), and } 7 \\
\text { (DOH adders appl ied to support from Others (abor). }\end{array}$ & & & $\$ 7.5 \mathrm{~K}$ & \\
\hline LEVEL 1 TOTALS & 11.0 & Ses Note & $\begin{array}{r}601.8 K \\
5609.5 K \\
\end{array}$ & $\begin{array}{l}\text { Het Originated Cost } \\
\text { Assessed Cost } \\
\text { Total Budget }\end{array}$ \\
\hline
\end{tabular}

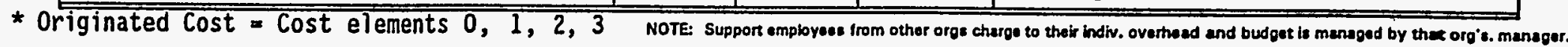




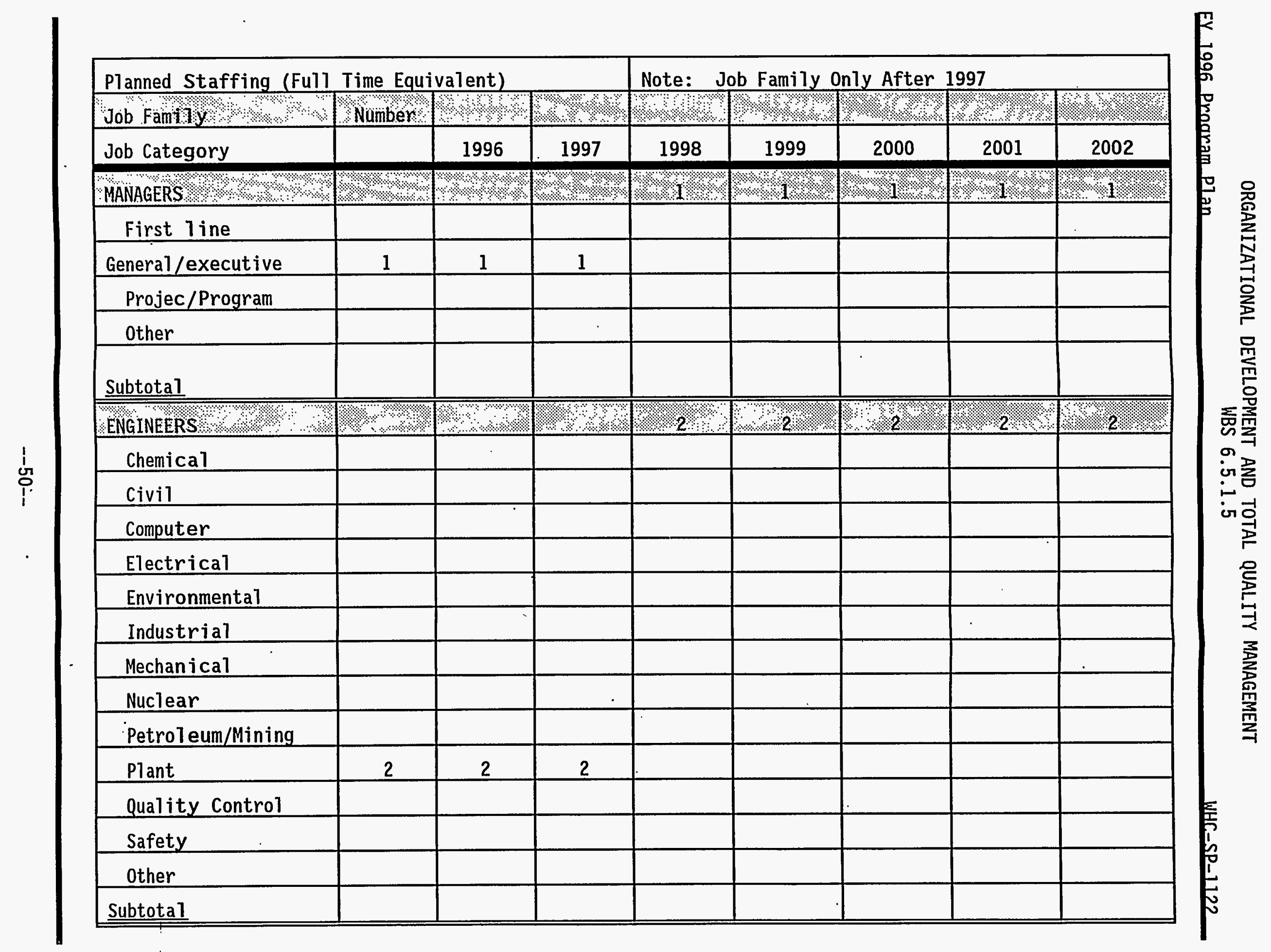




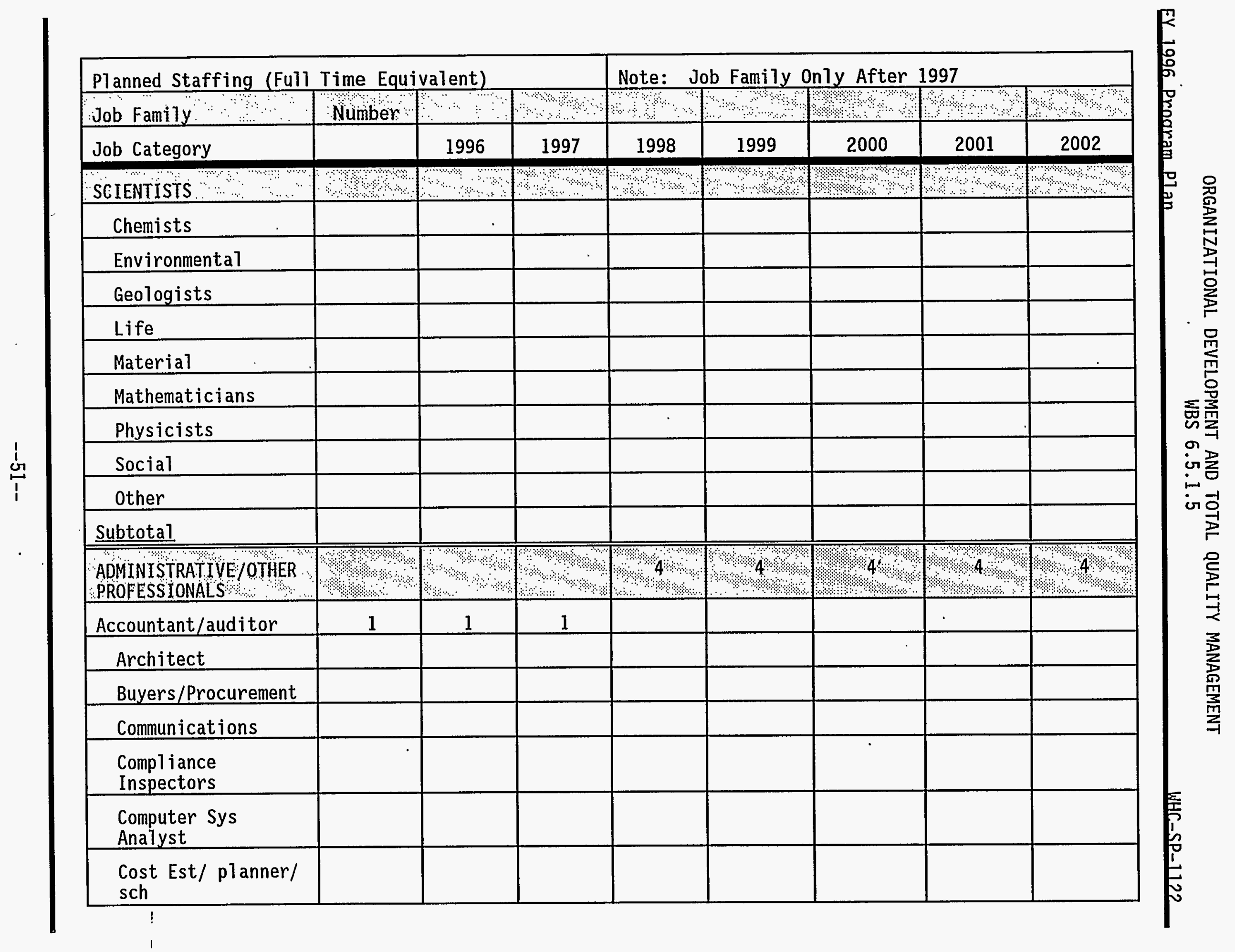




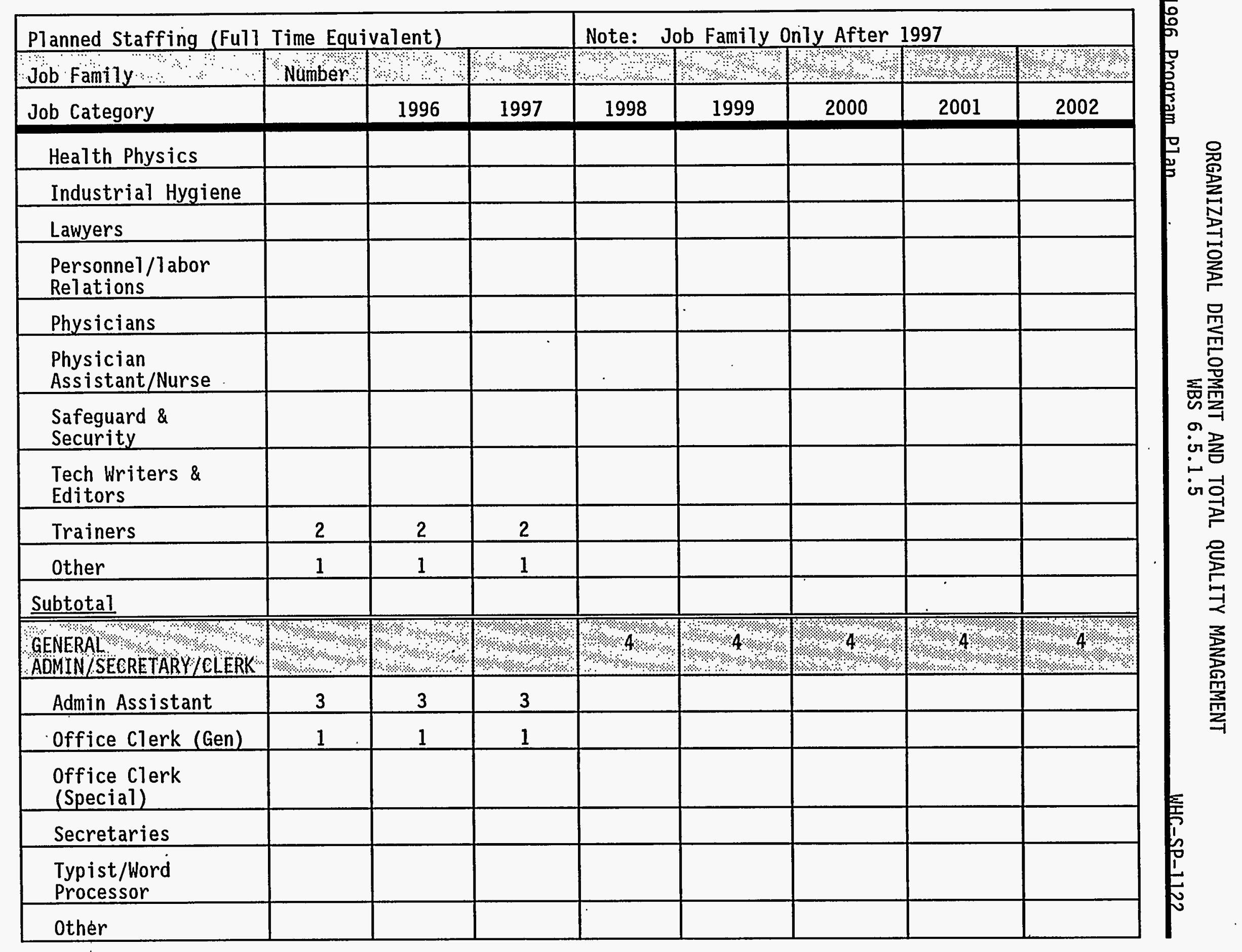




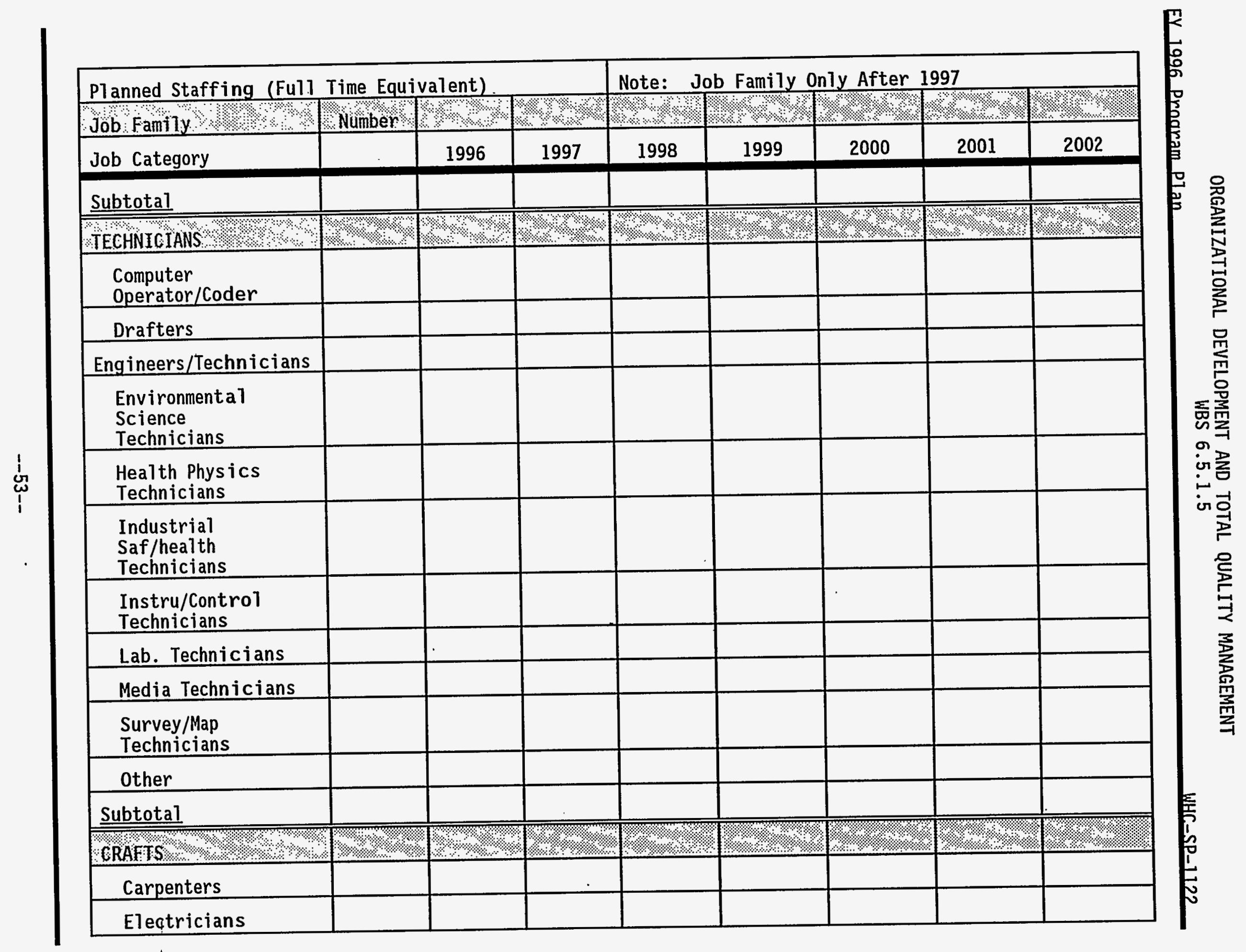




\begin{tabular}{|c|c|c|c|c|c|c|c|c|}
\hline \multicolumn{4}{|c|}{ Planned Staffing (Full Time Equivalent) } & \multicolumn{5}{|c|}{ Note: Job Family Only After 1997} \\
\hline Job Family & Number & 2. & $0<1$ & (2: 2 & (2) & 1.28 & 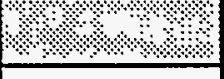 & 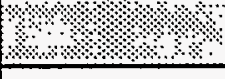 \\
\hline Job Category & & 1996 & 1997 & 1998 & 1999 & 2000 & 2001 & 2002 \\
\hline \multicolumn{9}{|l|}{ HVAC } \\
\hline \multicolumn{9}{|l|}{ Machinists } \\
\hline \multicolumn{9}{|l|}{ Masons } \\
\hline \multicolumn{9}{|l|}{ Millwrights } \\
\hline \multicolumn{9}{|l|}{ Painters } \\
\hline \multicolumn{9}{|l|}{ PImbrs/Pipefitters } \\
\hline \multicolumn{9}{|l|}{$\begin{array}{l}\text { Struct/Meta } 1 \\
\text { Workers }\end{array}$} \\
\hline \multicolumn{9}{|l|}{$\begin{array}{l}\text { Vehicl/Mobile } \\
\text { Equip. } \\
\text { Mechanics }\end{array}$} \\
\hline \multicolumn{9}{|l|}{ Welders } \\
\hline \multicolumn{9}{|l|}{ Other } \\
\hline \multicolumn{9}{|l|}{ Subtotal } \\
\hline OPERATORS & 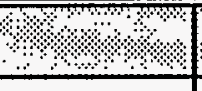 & 12. & $12=2.2$ & 12 & $12 \%$ & 1 & 12.2 & 1\% \\
\hline \multicolumn{9}{|l|}{ Lt. Vehicle Drivers } \\
\hline \multicolumn{9}{|l|}{$\begin{array}{l}\text { Material Moving } \\
\text { Equip. }\end{array}$} \\
\hline \multicolumn{9}{|l|}{ Nuclear Plant } \\
\hline \multicolumn{9}{|l|}{$\begin{array}{l}\text { Utilities Waste } \\
\text { Proces }\end{array}$} \\
\hline \multicolumn{9}{|l|}{ other } \\
\hline$\underline{\underline{\text { Subtotal }}}$ & & & & & & & & \\
\hline
\end{tabular}




\begin{tabular}{|c|c|c|c|c|c|c|c|c|}
\hline \multicolumn{4}{|c|}{ Planned Staffing (Full Time Equivalent) } & \multicolumn{5}{|c|}{ Note: Job Family OnTy After 1997} \\
\hline Job Family i & Number: & $\%$ & 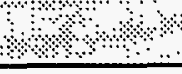 & ४ ? & ४४४ & १ै ४ै? & ?. & \%.।. \\
\hline Job Category & & 1996 & 1997 & 1998 & 1999 & 2000 & 2001 & 2002 \\
\hline $\begin{array}{l}\text { LABOR \& GENERAL } \\
\text { WORKERS }\end{array}$ & $8 \%$ & +4 & +2 & $2 \%$ & $1+4$ & \% & 2.8 & $\mathrm{H}^{2}+{ }^{2}+2$ \\
\hline Firefighters & & & & & & & & \\
\hline Food Service & & & & & & & & \\
\hline Hand/Help Lab Gen & & & & & & & & \\
\hline $\begin{array}{l}\text { Hand/Help Lab } \\
\text { Special }\end{array}$ & & & & & & & & \\
\hline Janitors/Cleaners & & & & & & & & \\
\hline Laundry Workers & & & & & & & & \\
\hline Security Guards & & & & & & & & \\
\hline Other & & & & & & & & \\
\hline Subtotal & & & & & & & & \\
\hline TOTAL FTES & & & & & & & & \\
\hline
\end{tabular}


3.1.6 Administration (WBS 6.5.1.6)

3.1.6.1 Administration Management (WBS 6.5.1.6.1)

Mission

The mission of Administration Management is to manage Cost Estimating, Project Planning \& Controls, Finance, Contract Services and Prime Contract Administration.

Product/Services Provided

The Management Administration provides services to the Administration Department in the areas of: ICF KH Policy and Procedures, Performance Standards, Financial policy, and Salary administration.

Primary Customers

Primary Customers of the Administration Management are: ICF Kaiser Hanford, ICF Kaiser Corporate, Westinghouse Hanford Company, U.S. Department of Energy (RL \& HQ), Boeing Computer Services, Pacific Northwest Laboratory, and Bechtel Hanford, Incorporated. 


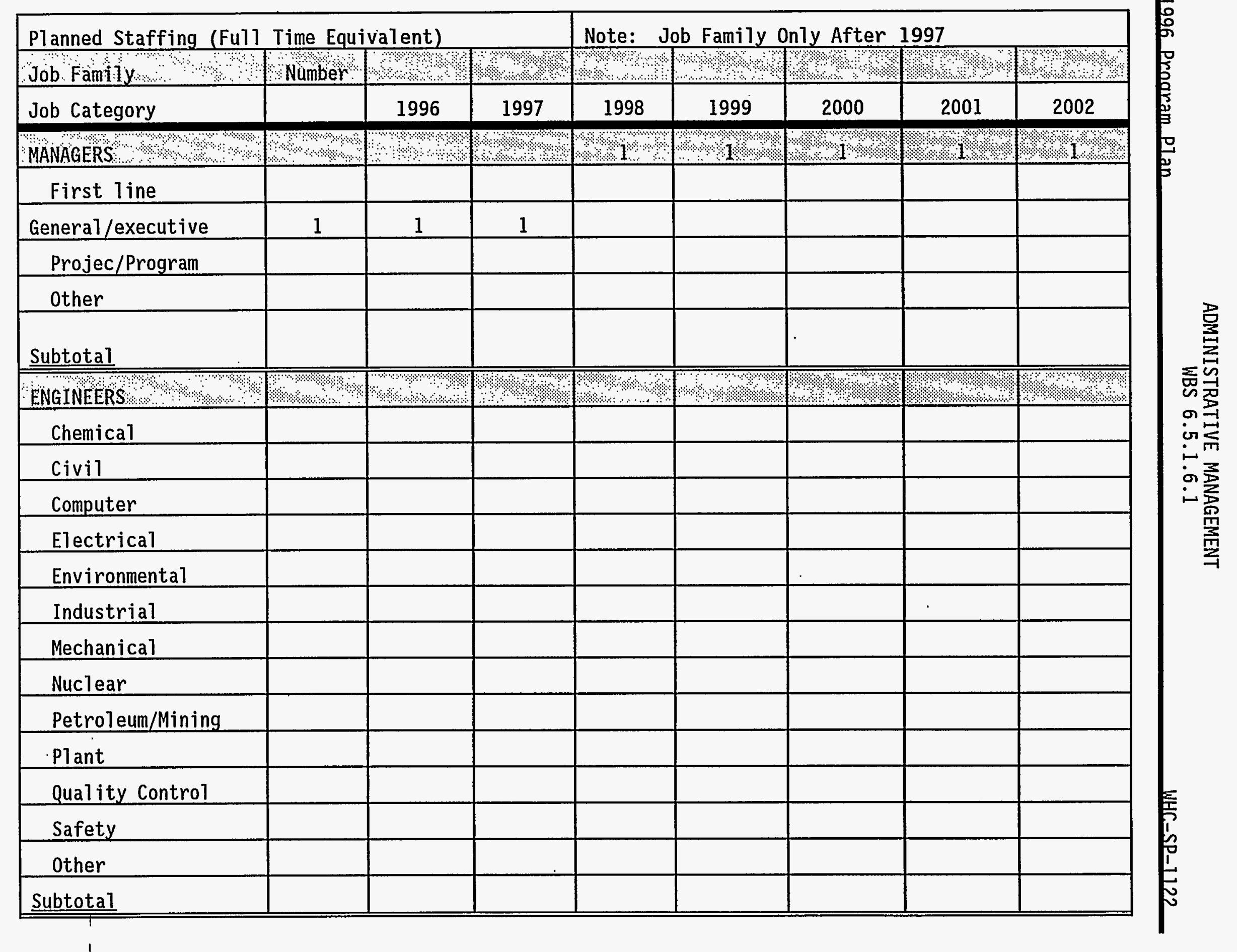




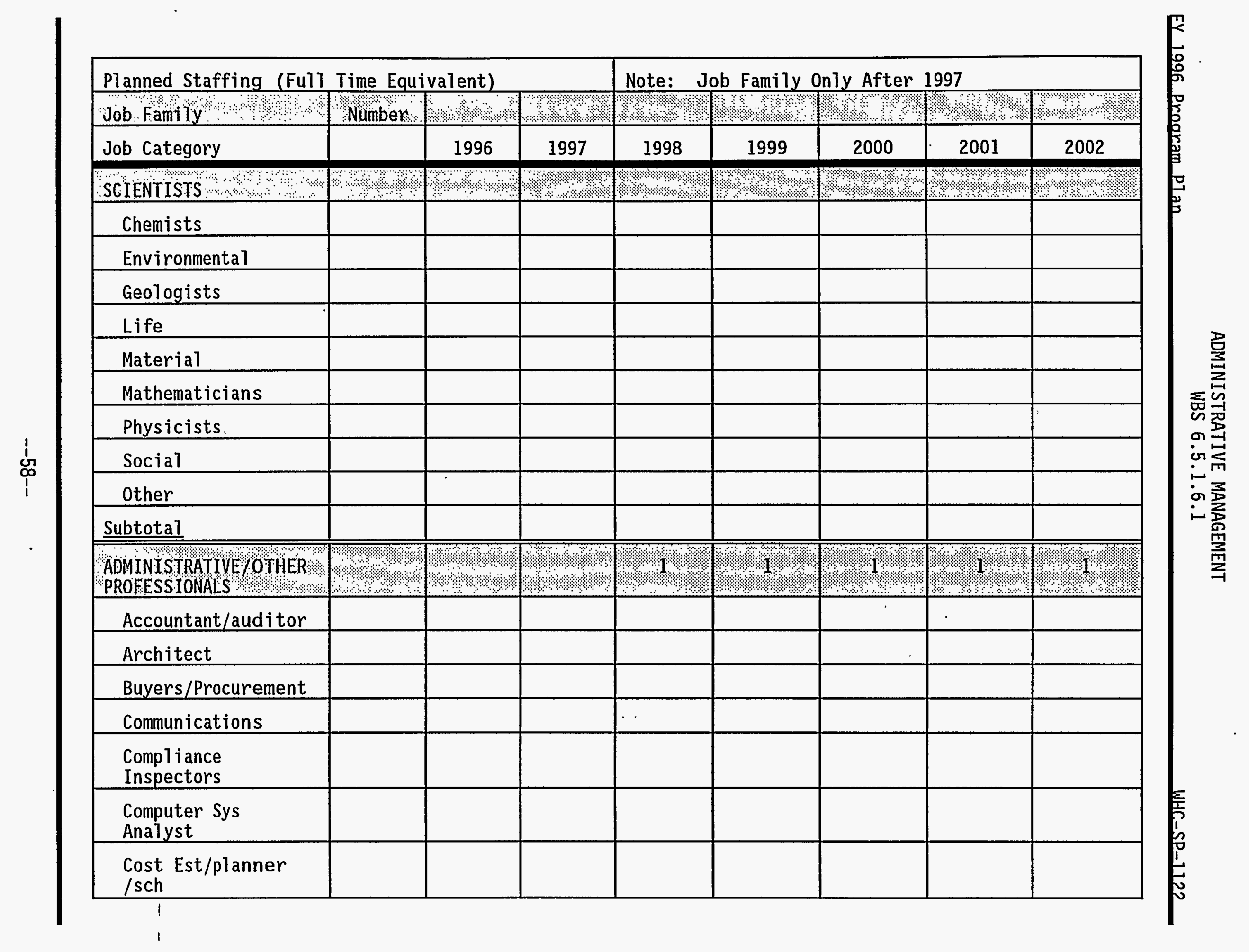




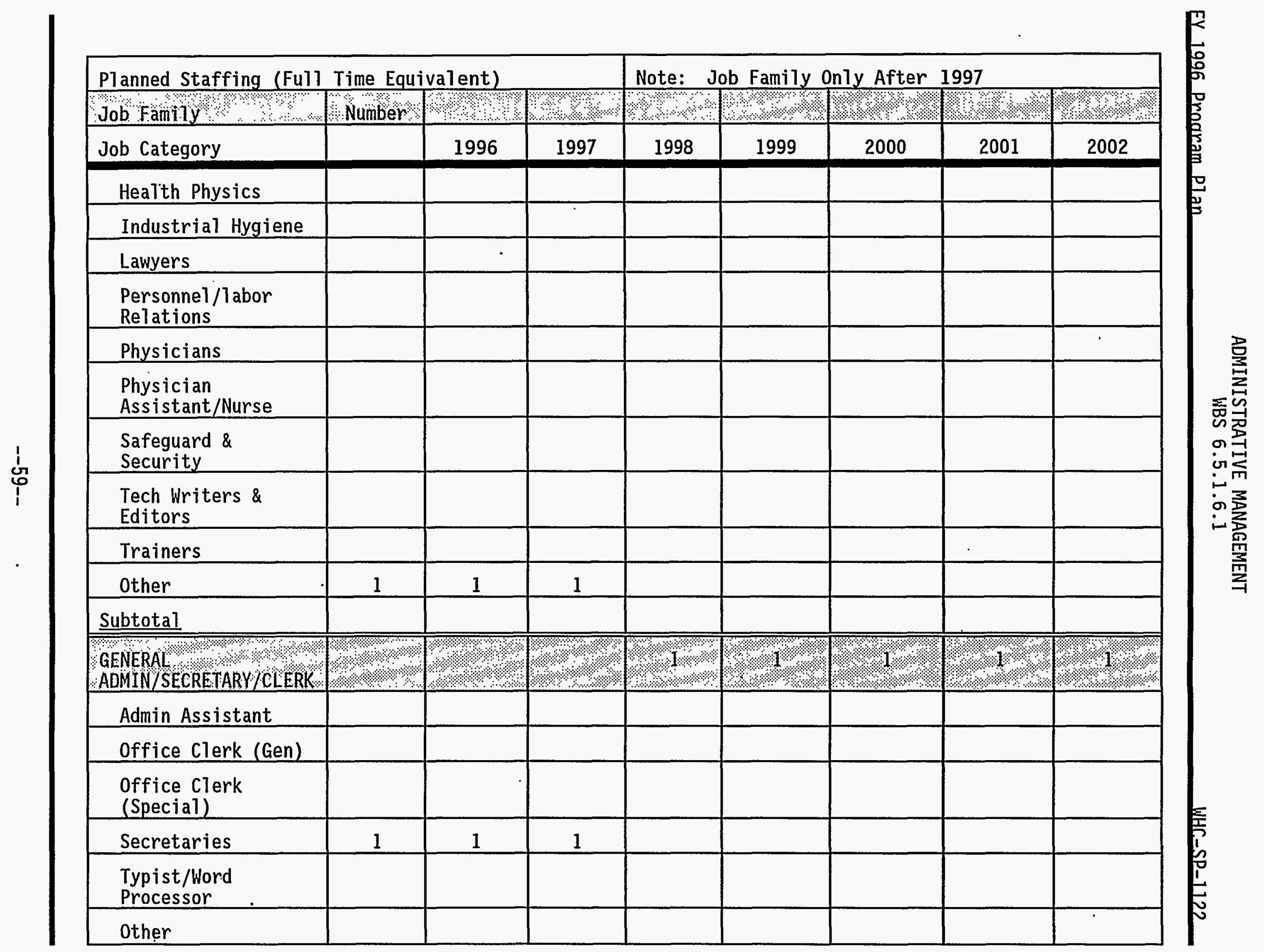




\begin{tabular}{|c|c|c|c|c|c|c|c|c|}
\hline \multicolumn{4}{|c|}{ Planned Staffing (Full Time Equivalent) } & \multicolumn{5}{|c|}{ Note: Job Family Only After 1997} \\
\hline Job Fam & Number: & ४४४ঃ: & \% \% & ८४०० & ४ै। & $1.4 \%$ & ४४९े। & $1 \%$ \\
\hline Job Category & & 1996 & 1997 & 1998 & 1999 & 2000 & 2001 & 2002 \\
\hline Subtotal & & & & & & & & \\
\hline TECHNICIANS & ४४४ & \%४: & \%? ? & \%? & 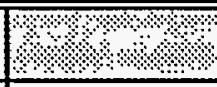 & \%? & 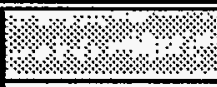 & (1\%?\%? \\
\hline $\begin{array}{l}\text { Computer } \\
\text { Operator/Coder }\end{array}$ & & & & & & & & \\
\hline Drafters & & & & & & & & \\
\hline Engineers/Technicians & & & & & & & & \\
\hline $\begin{array}{l}\text { Environmental } \\
\text { Science } \\
\text { Technicians }\end{array}$ & & & & & & & & \\
\hline $\begin{array}{l}\text { Health Physics } \\
\text { Technicians }\end{array}$ & & & & & & & & \\
\hline $\begin{array}{l}\text { Industrial } \\
\text { Saf/health } \\
\text { Technicians } \\
\end{array}$ & & & & & & & & \\
\hline $\begin{array}{l}\text { Instru/Control } \\
\text { Technicians }\end{array}$ & & & & & & & & \\
\hline Lab. Technicians & & & & & & & & \\
\hline Media Technicians & & & & & & & & \\
\hline $\begin{array}{l}\text { Survey/Map } \\
\text { Technicians }\end{array}$ & & & & & & & & \\
\hline Other & & & & & & & & \\
\hline Subtotal & & & & & & & & \\
\hline CRAFTS ... & अ. & $\%$ & \%ै.े. & $\% \%$ & $\% \% \%$ & $1+\%$ & $\% \div \%$ & $1 \% \%$ \\
\hline Carpenters & & & & & & & & \\
\hline
\end{tabular}




\begin{tabular}{|c|c|c|c|c|c|c|c|c|}
\hline \multicolumn{4}{|c|}{ Planned Staffing (Full Time Equivalent) } & \multicolumn{5}{|c|}{ Note: Job Family Only After 1997} \\
\hline Job Family & Number & \%? & \%०\% & \%? & $4.8 \%$ & 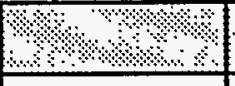 & ४४४४। & एथ \\
\hline Job Category & & 1996 & 1997 & 1998 & 1999 & 2000 & 2001 & 2002 \\
\hline \multicolumn{9}{|l|}{ Electricians } \\
\hline \multicolumn{9}{|l|}{ HVAC } \\
\hline \multicolumn{9}{|l|}{ Machinists } \\
\hline \multicolumn{9}{|l|}{ Masons } \\
\hline \multicolumn{9}{|l|}{ Millwrights } \\
\hline \multicolumn{9}{|l|}{ Painters } \\
\hline \multicolumn{9}{|l|}{ Plmbrs/Pipefitters } \\
\hline \multicolumn{9}{|l|}{$\begin{array}{l}\text { Struct/Metal } \\
\text { Workers }\end{array}$} \\
\hline \multicolumn{9}{|l|}{$\begin{array}{l}\text { Vehicl/Mobile } \\
\text { Equip. } \\
\text { Mechanics }\end{array}$} \\
\hline \multicolumn{9}{|l|}{ Welders } \\
\hline \multicolumn{9}{|l|}{ Other } \\
\hline \multicolumn{9}{|c|}{\begin{tabular}{l|l|l|l|l|l|l|l|} 
Subtotal & & & & & & & \\
\end{tabular}} \\
\hline OPERATORS & $3 \% 2$ & \%०े. & 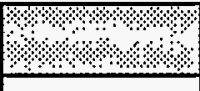 & $\%$ & $1 \% \%$ & \%.+. & $2 \%$ & $\%$ \%.?. \\
\hline \multicolumn{9}{|l|}{ Lt. Vehicle Drivers } \\
\hline \multicolumn{9}{|l|}{$\begin{array}{l}\text { Material Moving } \\
\text { Equip. }\end{array}$} \\
\hline \multicolumn{9}{|l|}{ Nuclear Plant } \\
\hline \multicolumn{9}{|l|}{$\begin{array}{l}\text { Utilities Waste } \\
\text { Proces }\end{array}$} \\
\hline \multicolumn{9}{|l|}{ Other } \\
\hline Subtotal & & & & & & & & \\
\hline
\end{tabular}




\begin{tabular}{|c|c|c|c|c|c|c|c|c|}
\hline \multicolumn{4}{|c|}{ Planned Staffing (Full Time Equivalent) } & \multicolumn{5}{|c|}{ Note: Job Family Only After 1997} \\
\hline Job Famity & Number: & \%?: & \%?:\% & $3 \% \%$ & \%? & 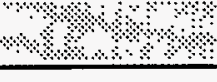 & 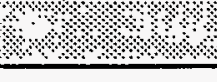 & ४०े \\
\hline Job Category & & 1996 & 1997 & 1998 & 1999 & 2000 & 2001 & 2002 \\
\hline $\begin{array}{l}\text { CABOR \& GENERAL } \\
\text { WORKERS }\end{array}$ & \%०े & अि\%: & 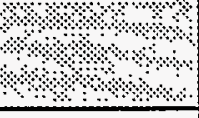 & \%? & \%ै०० & $3^{3}+2$ & 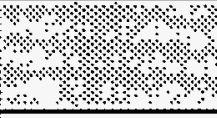 & ?ै. \\
\hline \multicolumn{9}{|l|}{ Firefighters } \\
\hline \multicolumn{9}{|l|}{ Food Service } \\
\hline \multicolumn{9}{|l|}{ Hand/Help Lab Gen } \\
\hline \multicolumn{9}{|l|}{$\begin{array}{l}\text { Hand/Help Lab } \\
\text { Special }\end{array}$} \\
\hline \multicolumn{9}{|l|}{ Janitors/Cleaners } \\
\hline \multicolumn{9}{|l|}{ Laundry Workers } \\
\hline \multicolumn{9}{|l|}{ Security Guards } \\
\hline \multicolumn{9}{|l|}{ other } \\
\hline \multicolumn{9}{|l|}{ Subtotal } \\
\hline TOTAL FTES & & & & & & & & \\
\hline
\end{tabular}

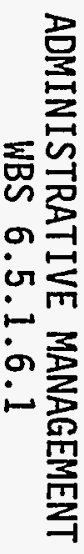




\subsubsection{Finance (WBS 6.5.1.6.2)}

$\underline{\text { Mission }}$

The mission of the Finance Department is to insure prudent management of government funds by the company, in performing contract defined workscope, and represent ICF Kaiser Hanford and it's employees in business related financial matters.

\section{Products/Services Provided}

The Finance Department performs the following services: Architect/Engineering and Construction labor pools payroll processing, Project and Contract Accounting, Contract payments, Operations and Maintenance Financial Support, Cost/Price Analysis, Time Card/TDR processing, and allowable cost management, and company level reporting.

\section{Primary Customers}

Primary Customers of the Finance Department are: ICF Kaiser Hanford, ICF Kaiser Engineers, ICF Kaiser International, U.S. Department of Energy (RL \& HQ), Westinghouse Hanford Company, Boeing Computer Services, Pacific Northwest Laboratory, and Bechtel Hanford Incorporated. 


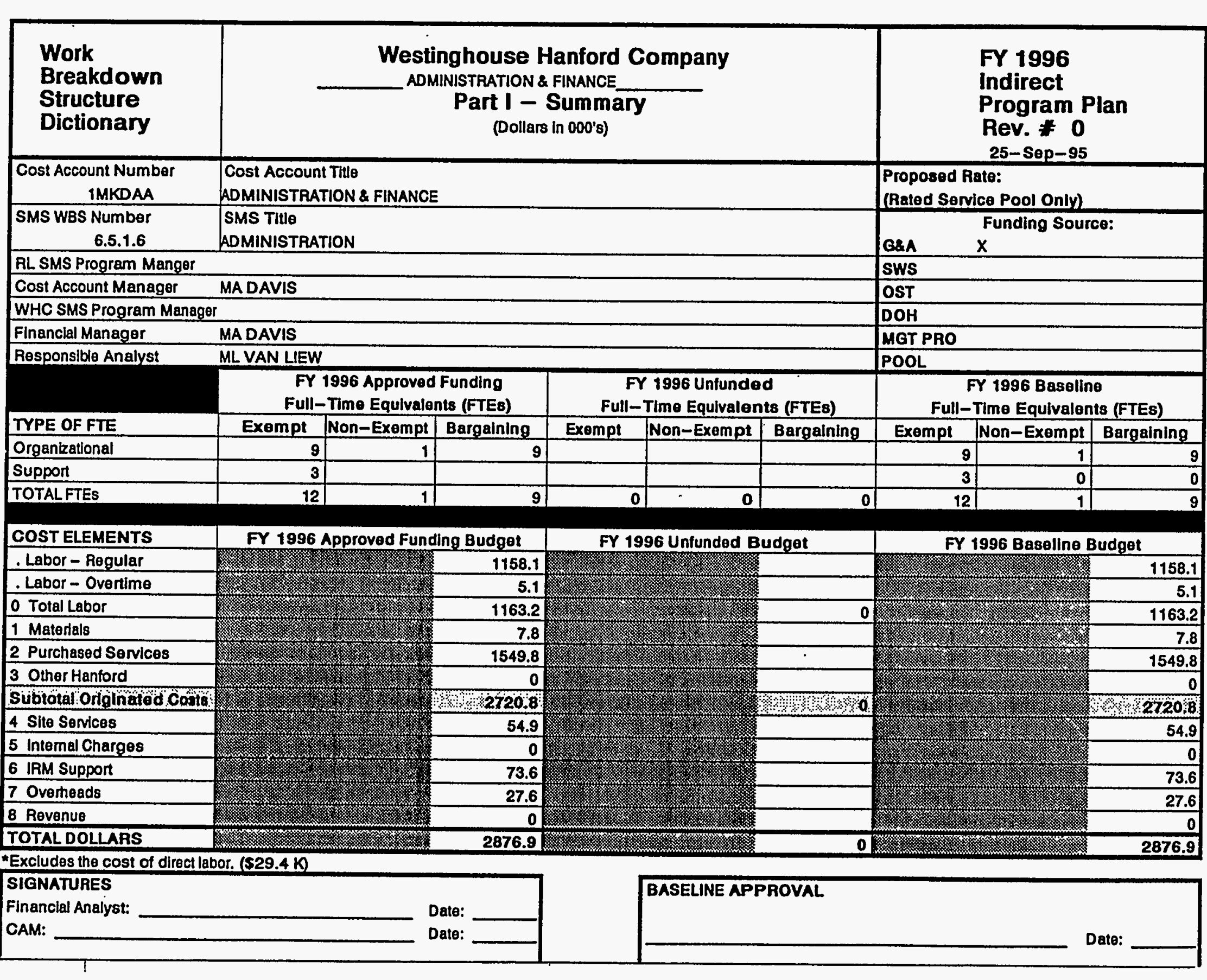




\begin{tabular}{|c|c|c|c|c|}
\hline \multicolumn{5}{|l|}{ BUILDING BLOCKS FOR FY 1996} \\
\hline \multicolumn{5}{|c|}{ FY 1996 Building Blocks (SO00) ADHINISTRATION \& FIMANCE: K.B. ADAHSON } \\
\hline $\begin{array}{l}\text { ORGANIZATIOWAL LORK SCOPE DESCRIPTIOW } \\
\text { (BUDGET.96 - IMAOMA) }\end{array}$ & $\begin{array}{l}\text { Org. FTEs } \\
\text { in } \\
\text { Workseope }\end{array}$ & $\begin{array}{c}\text { Support } \\
\text { rTes in } \\
\text { Horkscope }\end{array}$ & $\begin{array}{c}\text { FY } 1996 \\
\text { Originated } \\
\text { Cost" }\end{array}$ & $\begin{array}{l}\text { JUSTIFICATIOH OF SCOPE/IMPACT } \\
\text { If WOT FUNDED }\end{array}$ \\
\hline 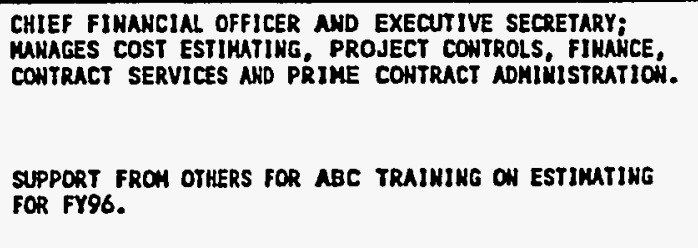 & 2 & 2 & $\$ 1119.4 \mathrm{~K}$ & 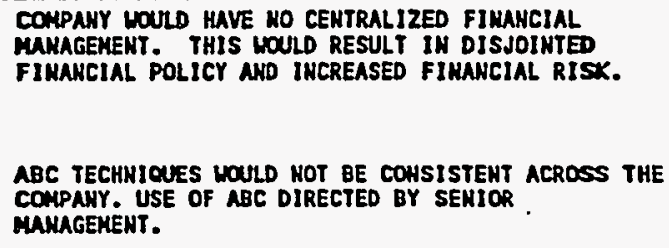 \\
\hline PRIME COHTRACT ADHIHISTRATION & 1 & 0 & $\$ 76.5$ & $\begin{array}{l}\text { PRIME CONTRACT COHPLIANCE COULD NOT BE MONITORED. } \\
\text { PRESENTS FIMANCIAL RISK TO THE COMPANY IF UW- } \\
\text { FUNDED. }\end{array}$ \\
\hline $\begin{array}{l}\text { ARCHITECT/ENGINEERING \& CONSTRUCTIOW LABOR POOLS } \\
\text { PAYROLL PROCESSING }\end{array}$ & 2 & 0 & $\$ 69.2$ & EMPLOYEES HOULD MOT GET PAID. \\
\hline 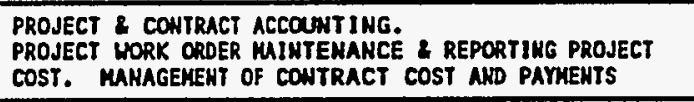 & 5.25 & 0 & 227.7 & $\begin{array}{l}\text { If UMFUHDED, PROJECT MAKAGERS WOULD HAVE GREAT } \\
\text { DIFFICULTY MHAGIKG JOB COST. }\end{array}$ \\
\hline COHTRACT PAYMENTS & 1 & 0 & 34.0 & 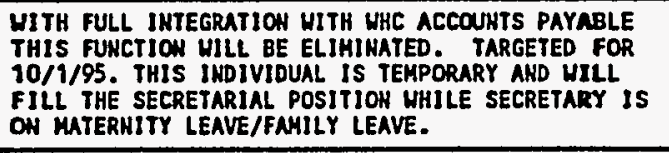 \\
\hline COST/PRICE AHALYSIS. & 1 & 0 & 45.0 & 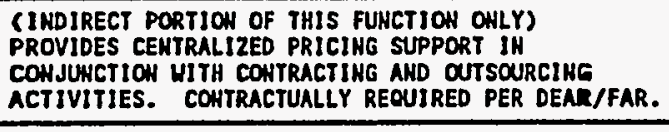 \\
\hline $\begin{array}{l}\text { GEA, A/EC LABOR POOL BLOGETIHG AND RECOVERY } \\
\text { MHAGGEENT }\end{array}$ & 1.5 & 0 & 110.3 & 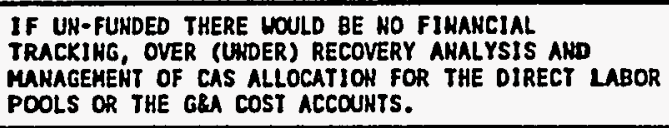 \\
\hline 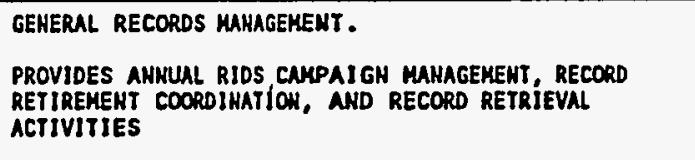 & 0 & 1 & 107.8 & $\begin{array}{l}\text { If UHFUNDED THERE HOULD BE HO CEHTRALIZED RECORDS } \\
\text { RAHAGEMENT OR COORDIHATION AND TRAIHIHG ON } \\
\text { RECORDS RETEHTIOH REQUIREHEHTS. }\end{array}$ \\
\hline
\end{tabular}


BUILDING BLOCKS FOR FY 1996

FY 1996 Building Blocks (S000) ADKIMISTRATIOW \& FIKANCE: K.B. ADMHSOW

\begin{tabular}{|c|c|c|c|c|}
\hline $\begin{array}{l}\text { ORGAHIZATIOHAL WORK SCOPE DESCRIPTIOU } \\
\text { (BLOGET.96 - IMKDMA) }\end{array}$ & $\begin{array}{l}\text { Org. FiEs } \\
\text { in } \\
\text { Workscope }\end{array}$ & $\begin{array}{l}\text { Support } \\
\text { FTEs in } \\
\text { Morkscope }\end{array}$ & $\begin{array}{c}\text { FY } 1996 \\
\text { originated } \\
\text { Cost* }\end{array}$ & $\begin{array}{l}\text { JUSTIFICATION OF SCOPE/IKPACT } \\
\text { IF NOT FUNDED }\end{array}$ \\
\hline PAYKENT OF DCM FINAL AUDIT RATE ADJUSTMEHTS & 0 & 0 & 181.2 & $\begin{array}{l}\text { THIS IS FOR PAYMEKT OF FINAL AFFILIATE DCMA } \\
\text { APPROVED RATES. THESE RATES ARE HOT APPROUED OR } \\
\text { BILLED UWITL A SICHIFICANT PERIOD AFTER THE CLOSE } \\
\text { OF THE ORIGIWAL PROJECT THAT REQUIRED THE } \\
\text { SUPPORT. }\end{array}$ \\
\hline $\begin{array}{c}\text { TIME CARD/TOR PROCESSING (A/EC LABOR POOLS) } \\
\text { C }\end{array}$ & 2 & 0 & 67.0 & $\begin{array}{l}\text { WITH FULL IKPLEMEHTATIOH OF "TIS" THIS FUNCTION } \\
\text { WILL BE REDUCED HITH REMAIHING BUILDING TRNDES } \\
\text { TIMECARD/TDR PROCESSING YO BE ABSORBED INTO THE } \\
\text { PROJECT ACCOWWTING FUHCTION. } \\
\end{array}$ \\
\hline $\begin{array}{l}\text { UNALLOMABLE COST MANAGEHENT AND CONPANY LEVEL } \\
\text { REPORTING }\end{array}$ & 3.25 & 0 & 209.6 & $\begin{array}{l}\text { PAYMENT OF MOH-CONTRACT LIABILITIES-CONTRACTULAL } \\
\text { REQUIREMEMT, IF UN-FUHDED THERE WOULD BE DO } \\
\text { FINANCIAL REPORTING AT A TOTAL COHPANY LEVEL FOR } \\
\text { IHTERHAL USE OR FOR REPORTIHG TO CORPORATE. }\end{array}$ \\
\hline Taxes & & & 1159.1 & \\
\hline $\begin{array}{l}\text { ASSESSED COSTS: These include Cost El mments } 4 \text {. } \\
\text { (Services provided by IHC/ICFKH), } 5 \text { (Company level } \\
\text { services, ie. Dosinetry), } 6 \text { (IRH Services), and } 7 \\
\text { (DOH adders applied to support from others lebor). }\end{array}$ & & & 156.1 & \\
\hline LEVEL 1 TOTALS & 19 & 3 & $\begin{array}{l}\$ 2,545.8 \\
\$ 2,701.9\end{array}$ & $\begin{array}{l}\text { MET ORIGIMATED COST } \\
\text { ASSESSHENTS } \\
\text { TOTAL BUDGET }\end{array}$ \\
\hline
\end{tabular}

* Originated Cost $=$ Cost elements $0,1,2,3$ (DOLLARS IN THOUSANDS) 


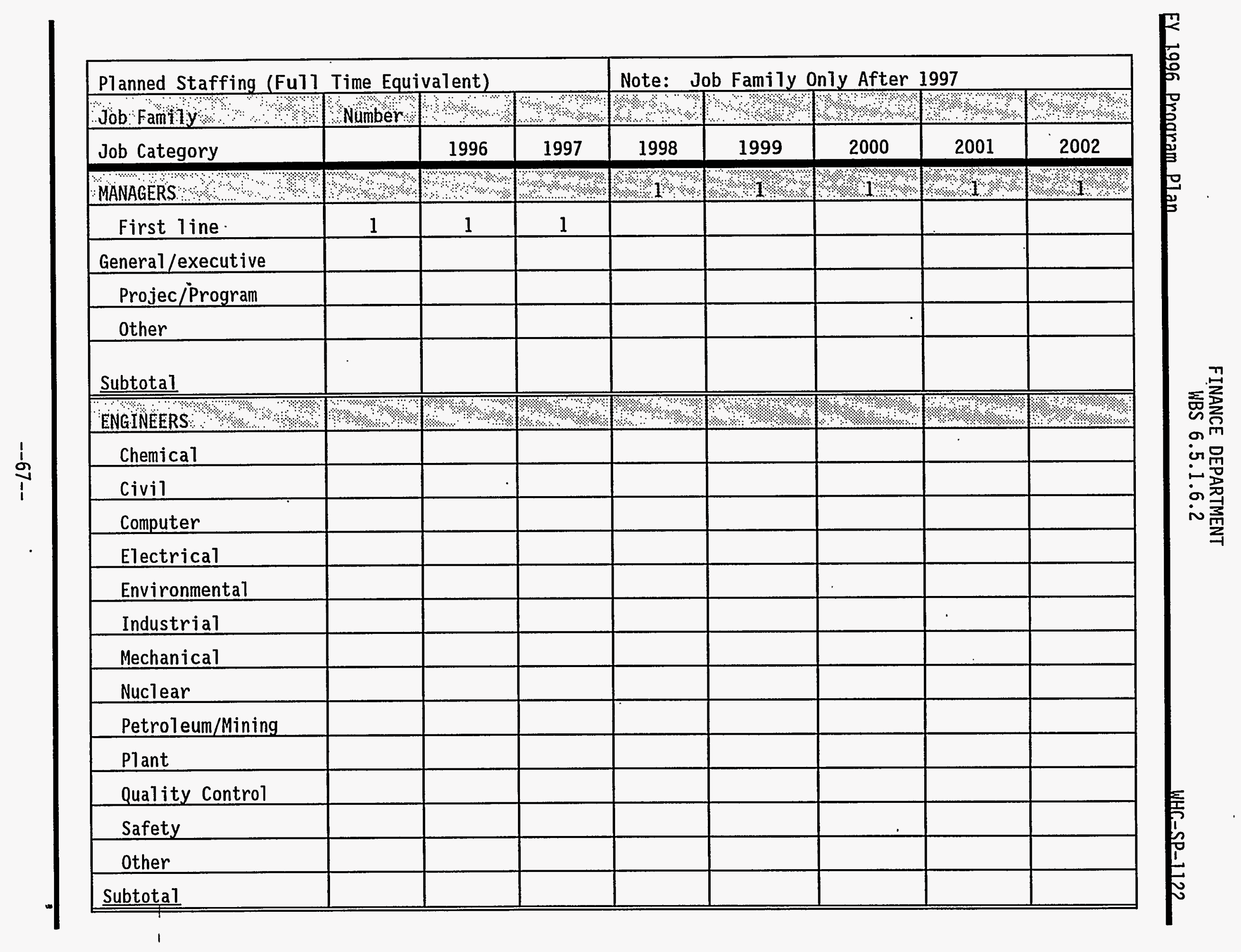




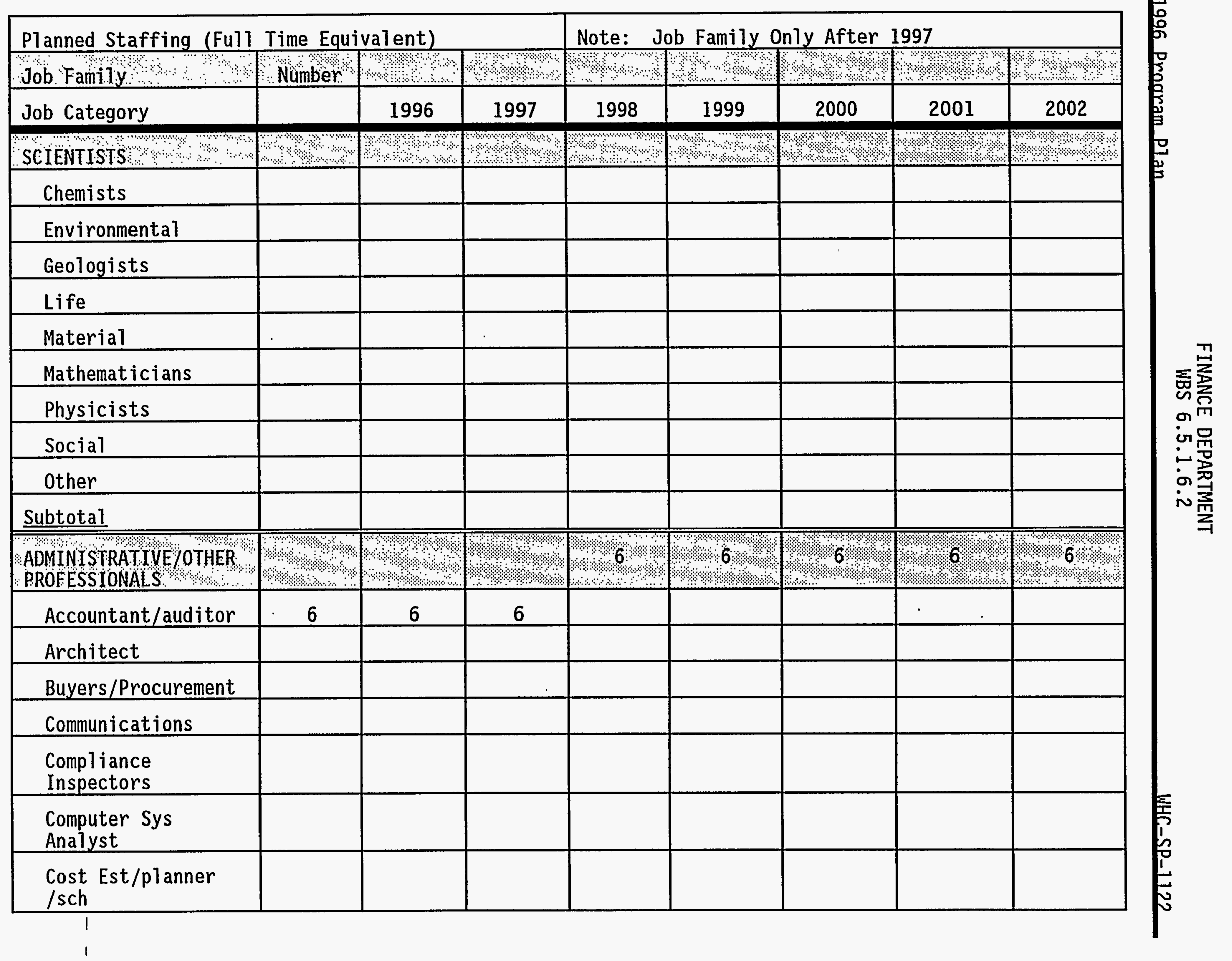




\begin{tabular}{|c|c|c|c|c|c|c|c|c|}
\hline Planned Staffing (Full & Time Equi & valent) & & Note: Jo & ob Family 0 & nily After & & \\
\hline Job Famfly & Number & अि & $4 \%$ & अ००। & $\%$ & 284 & 4. & \%०े \\
\hline Job Category & & 1996 & 1997 & 1998 & 1999 & & 2001 & 2002 \\
\hline Health Physics & & & & & & & & \\
\hline Industrial Hygiene & & & & & & & & \\
\hline Lawyers & & & & & & & & \\
\hline $\begin{array}{l}\text { Personnel/1abor } \\
\text { Relations }\end{array}$ & & & & & & & & \\
\hline Physicians & & & & & & & & \\
\hline $\begin{array}{l}\text { Physician } \\
\text { Assistant/Nurse }\end{array}$ & & & & & & & & \\
\hline $\begin{array}{l}\text { Safeguard \& } \\
\text { Security }\end{array}$ & & & & & & & & \\
\hline $\begin{array}{l}\text { Tech Writers \& } \\
\text { Editors }\end{array}$ & & & & & & & & \\
\hline Trainers & & & & & & & & \\
\hline Other & & & & & & & & \\
\hline Subtotal & & & & & & & & \\
\hline $\begin{array}{l}\text { GENERAL, } \\
\text { ADMIN/SECRETARY/CLERK }\end{array}$ & ४ै। & 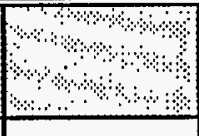 & ४ै। & अें & $3 \%$ & \% 9.9 & $1 . .9 \%$ & $1 \%$ \\
\hline Admin Assistant & & & & & & & & \\
\hline Office Clerk (Gen) & 9 & 9 & 9 & & & & & \\
\hline $\begin{array}{l}\text { Office Clerk } \\
\text { (Special) }\end{array}$ & & & & & & & & \\
\hline Secretaries & & & & & & & & \\
\hline $\begin{array}{l}\text { Typist/Word } \\
\text { Processor }\end{array}$ & & & & & & & & \\
\hline Other & & & & & & & & \\
\hline
\end{tabular}




\begin{tabular}{|c|c|c|c|c|c|c|c|c|}
\hline \multicolumn{4}{|c|}{ Planned Staffing (Ful1 Time Equivalent) } & \multicolumn{5}{|c|}{ Note: Job Family Only After 1997} \\
\hline Job Family,$\cdots$ & Number & 3 & $4 \%$ & \%४ & \%० & \%०४? & \%४ & \%४४? \\
\hline Job Category & & 1996 & 1997 & 1998 & 1999 & 2000 & 2001 & 2002 \\
\hline \multicolumn{9}{|l|}{ Subtotal } \\
\hline TECHNICIANS & +. & \%? & \%? & \%० & \%. & ४ै। & ४ং ২ ২ & 18 \\
\hline \multicolumn{9}{|c|}{\begin{tabular}{l|l|l} 
Computer & & \\
Operator/Coder & & \\
\end{tabular}} \\
\hline \multicolumn{9}{|l|}{ Drafters } \\
\hline \multicolumn{9}{|l|}{ Engineers/Technicians } \\
\hline \multicolumn{9}{|l|}{$\begin{array}{l}\text { Environmental } \\
\text { Science } \\
\text { Technicians }\end{array}$} \\
\hline \multicolumn{9}{|l|}{$\begin{array}{l}\text { Health Physics } \\
\text { Technicians }\end{array}$} \\
\hline \multicolumn{9}{|l|}{$\begin{array}{l}\text { Industrial } \\
\text { Saf/health } \\
\text { Technicians }\end{array}$} \\
\hline \multicolumn{9}{|l|}{$\begin{array}{l}\text { Instru/Control } \\
\text { Technicians }\end{array}$} \\
\hline \multicolumn{9}{|l|}{ Lab. Technicians } \\
\hline \multicolumn{9}{|l|}{ Media Technicians } \\
\hline \multicolumn{9}{|l|}{$\begin{array}{l}\text { Survey/Map } \\
\text { Technicians }\end{array}$} \\
\hline \multicolumn{9}{|l|}{ Other } \\
\hline \multicolumn{9}{|l|}{ Subtotal } \\
\hline CRAFTS $\%$ \% । & $1 \% . \%$ & \%. & $1 \% \%$ & \%० \% & \%. & 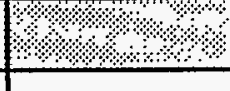 & $1 . \% 2 \%$ & $1 . \% \%$ \\
\hline Carpenters & & & & & & & & \\
\hline
\end{tabular}




\begin{tabular}{|c|c|c|c|c|c|c|c|c|}
\hline \multicolumn{4}{|c|}{ Planned Staffing (Full Time Equivalent) } & \multicolumn{5}{|c|}{ Note: Job Family Only After 1997} \\
\hline Job Family & Number: & 3 & बै। & as & « & अ०े। & अे। & धی \\
\hline Job Category & & 1996 & 1997 & 1998 & 1999 & 2000 & 2001 & 2002 \\
\hline \multicolumn{9}{|l|}{ Electricians } \\
\hline \multicolumn{9}{|l|}{ HVAC } \\
\hline \multicolumn{9}{|l|}{ Machinists } \\
\hline \multicolumn{9}{|l|}{ Masons } \\
\hline \multicolumn{9}{|l|}{ Millwrights } \\
\hline \multicolumn{9}{|l|}{ Painters } \\
\hline \multicolumn{9}{|l|}{ Plmbrs/Pipefitters } \\
\hline \multicolumn{9}{|l|}{$\begin{array}{l}\text { Struct/Metal } \\
\text { Workers }\end{array}$} \\
\hline \multicolumn{9}{|l|}{$\begin{array}{l}\text { Vehicl/Mobile } \\
\text { Equip! } \\
\text { Mechanics }\end{array}$} \\
\hline \multicolumn{9}{|l|}{ Welders } \\
\hline \multicolumn{9}{|l|}{ other } \\
\hline \multicolumn{9}{|l|}{ Subtotal } \\
\hline OPERATORS & $2 \%$ & ४ै। & \%) & \%० : & \%० & ४० \% & \%०० & \%? \\
\hline \multicolumn{9}{|l|}{ Lt. Vehicle Drivers } \\
\hline \multicolumn{9}{|l|}{$\begin{array}{l}\text { Material Moving } \\
\text { Equip. }\end{array}$} \\
\hline \multicolumn{9}{|l|}{ Nuclear Plant } \\
\hline \multicolumn{9}{|l|}{$\begin{array}{l}\text { Utilities Waste } \\
\text { Proces }\end{array}$} \\
\hline \multicolumn{9}{|l|}{ other } \\
\hline Subtotal & & & & & & . & & \\
\hline
\end{tabular}




\begin{tabular}{|c|c|c|c|c|c|c|c|c|}
\hline \multicolumn{4}{|c|}{ Planned Staffing (Full Time Equivalent) } & \multicolumn{5}{|c|}{ Note: Job Family Only After 1997} \\
\hline Job Family & Number. & $2 \ldots$ & ? & & एक४⿻ & ఋ४ & \%. & \% \\
\hline Job Category & & 1996 & 1997 & 1998 & 1999 & 2000 & 2001 & 2002 \\
\hline $\begin{array}{l}\text { LABOR \& GENERAL } \\
\text { WORKERS }\end{array}$ & $\mathrm{s}_{4}$ & $2 \times$ & + & & ४ & ४ै०ै? & +2 & 3 \\
\hline Firefighters & & & & & & & & \\
\hline Food Service & & & & & & & & \\
\hline Hand/Help Lab Gen & & & & & & & & \\
\hline $\begin{array}{l}\text { Hand/Help Lab } \\
\text { Special }\end{array}$ & & & & & & & & \\
\hline Janitors/Cleaners & & & & & & & & \\
\hline Laundry Workers & & & & & & & & \\
\hline Security Guards & & & & & & & & \\
\hline Other & & & & & & & & \\
\hline Subtotal & & & & & & & & \\
\hline TOTAL FTES & & & & & & & & \\
\hline
\end{tabular}




\section{GENERAL AND ADMINISTRATIVE \\ WBS 6.5.1}

WHC-SP-1122

3.1.7 Engineering and Technical Support Services (WBS 6.5.1.7)

3.1.7.1 Environmental Programs (WBS 6.5.1.7.1)

$\underline{\text { Mission }}$

The mission of the Environmental Programs and Integration Department is to formulate and formalize the policies, plans, procedures and documentation that constitutes the ICF KH Environmental Protection Program.

\section{Products/Services Provided}

The Environmental Programs and Integration Department provides the following services:

- Compliance Standards and Systems: Formulate and formalize the policies, plans, reports, management systems, and procedures for ICF KH compliance with environmental protection laws and requirements in areas such as pollution prevention, waste categorization, waste management, liquid effluents, airborne effluents, PCB management, etc.

- Environmental Compliance: Provide point of contact for coordination and resolution of environmental issues and represent ICF KH in discussions with regulators, DOE, WHC, and others.

- Technical Support: Help personnel in line organizations develop expertise. Mentor and assist personnel to gain environmental training, experience and credentials.

- Information and Documentation: Review environmental documents. Maintain a tracking system to document adherence to requirements for NEPA, SEPA, Cultural Resource Review, and Ecological Resources Reviews.

- $\quad$ Risk Management: Assist top management in minimizing liability by identifying risks and implementing risk management strategies.

Primary Customer

The primary customer of the Environmental Programs and Integration Department is ICF Kaiser Hanford. 


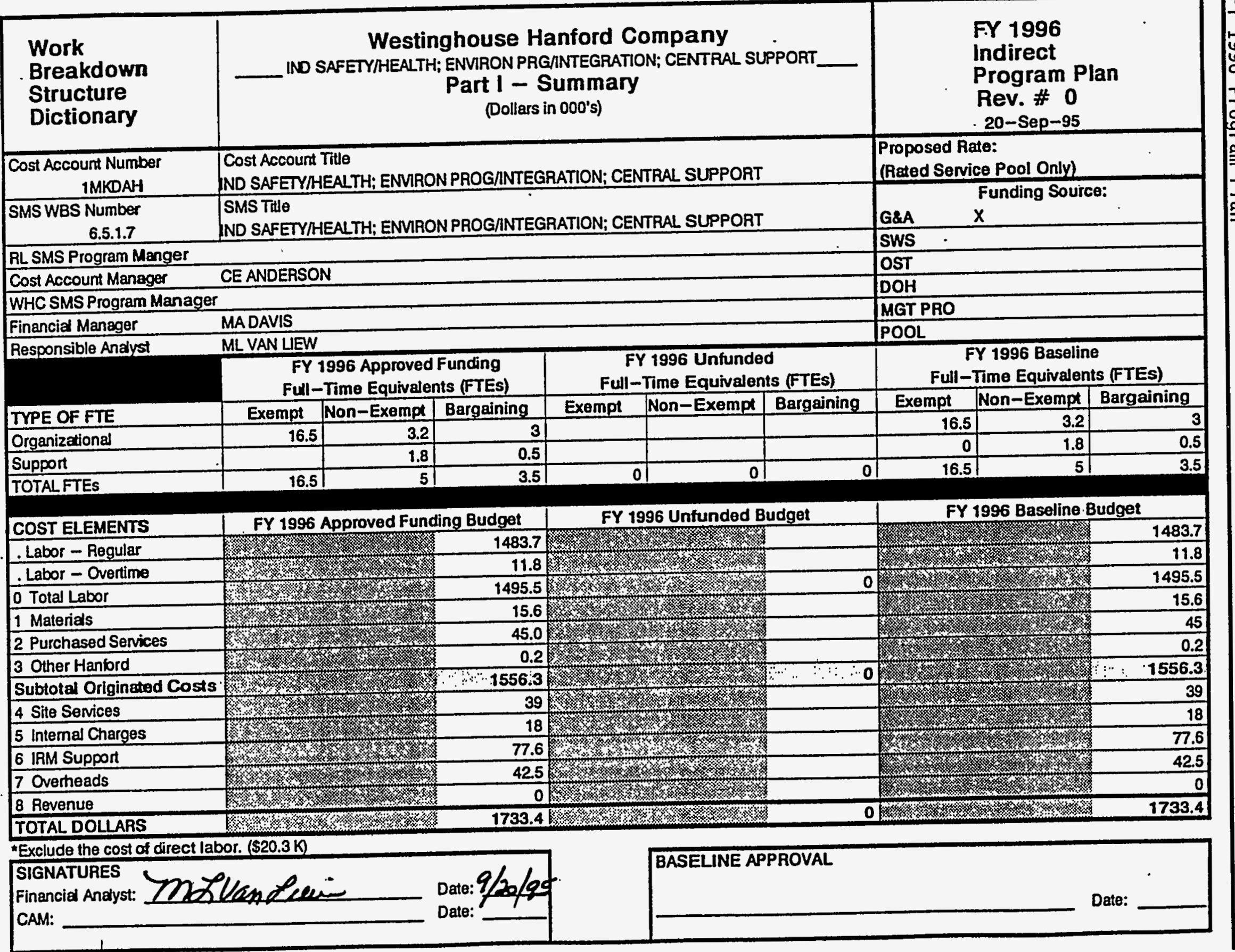


FY 1996 Building Blocks

Environmental Programs and Integration

\begin{tabular}{|c|c|c|c|c|}
\hline $\begin{array}{l}\text { ORGANIZATIOHAL WORK SCOPE DESCRIPTIOH } \\
\text { (budget95.6b - Part of 1MKOAH) }\end{array}$ & $\begin{array}{l}\text { Org. FTEs } \\
\text { in } \\
\text { Workscope }\end{array}$ & $\begin{array}{l}\text { Support } \\
\text { FTEs in } \\
\text { Workscope } \\
\end{array}$ & $\begin{array}{l}\text { Fy } 1996 \\
\text { Originated } \\
\text { Cost }\end{array}$ & JUSTIFICATION OF SCOPE/IMPACT IF NOT FUNDED \\
\hline 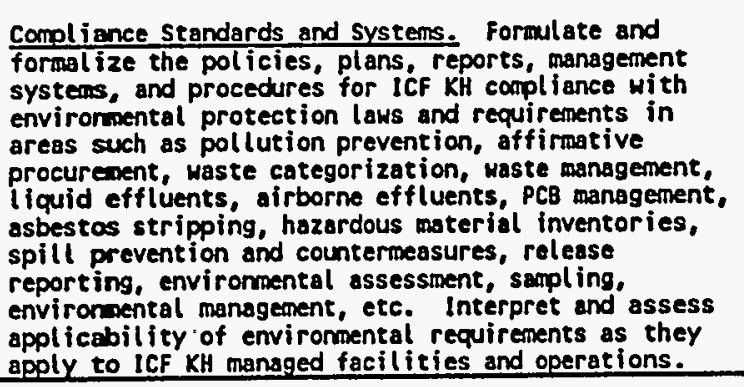 & 3.5 & 0 & 236.9 & $\begin{array}{l}\text { Without an enviromental progran, ICF KH will be } \\
\text { at risk of failure to satisfy compliance } \\
\text { obligations because of requirements may not be } \\
\text { identified and effective compl iance systems } \\
\text { developed to ensure compl iance. The lack of } \\
\text { standards of the development of several standards } \\
\text { within the conpany will cause confusion and will } \\
\text { raise costs due to dupl ication of effort or due to } \\
\text { penalties associated with nonconpl (iance. The } \\
\text { benefits of pollution prevention opportinities } \\
\text { (cost savings, minimization of liability, reduced } \\
\text { human exposure, etc.) will also be missed. }\end{array}$ \\
\hline $\begin{array}{l}\text { Enviromental Compliance officer. Provide an } \\
\text { Enviromental Compliance Officer to serve as a } \\
\text { centrat point of contact for coordination and } \\
\text { resolution of environmental issues and to represent } \\
\text { ICF XH in discussions with regulators, DOE, WHC, and } \\
\text { others. }\end{array}$ & 0.5 & 0 & 36.8 & $\begin{array}{l}\text { An Envirormental Compl iance officer (ECO) } \\
\text { represents the company on compl iance issues and } \\
\text { focuses on methods to prevent enviromental } \\
\text { noncompl iances. The ECO can serve as the } \\
\text { "conscience" of the company in identifying and } \\
\text { resolving noncompliances. One of the ECO's } \\
\text { primary responsibilities is to minimize } \\
\text { environgental liabilities of ICF KH and its } \\
\text { management and employees. }\end{array}$ \\
\hline $\begin{array}{l}\text { Technical support. Advise and assist ICF KH } \\
\text { personnel and organizations in achieving and } \\
\text { maintaining environmental compl iance. Help personnel } \\
\text { in line organizations develop expertise by mentoring } \\
\text { and assisting persomel in gaining the training, } \\
\text { experience, and credentials necessary to ensure } \\
\text { compl iance. Provide technical direction and } \\
\text { functional base for enviromental coordinators and } \\
\text { professionals. }\end{array}$ & 1.0 & 0 & 73.5 & $\begin{array}{l}\text { In a company with diverse enviromental } \\
\text { obligations, it is effective to hove a central } \\
\text { organization with the necessary knowledge, skills, } \\
\text { and experience to provide technical support to } \\
\text { line organizations. This approach reduces } \\
\text { confusion and is cost-effective. It also ensures } \\
\text { consistency throughout the company which reduces } \\
\text { liability and makes it easier for personnel } \\
\text { transferring between organizations. }\end{array}$ \\
\hline $\begin{array}{l}\text { Information and Documentation. Respond to requests } \\
\text { for information. Review environmental documents. } \\
\text { Maintain a tracking system document acherence to } \\
\text { requi rements for NEPA, SEPA, Cul tural Resource } \\
\text { Reviews, and Ecological Resources Revieks. Assist in } \\
\text { gathering and providing information needed for } \\
\text { permi ts or environtental documentation. Inform top } \\
\text { mansgeant about the compl iance status of ICF KH. }\end{array}$ & 1.0 & 0 & 73.5 & $\begin{array}{l}\text { Numerous requests are received by ICF KH asking } \\
\text { for information about compl lance status, for } \\
\text { responses to surveys, for reviews of new } \\
\text { enviromental requirements, etc. A central } \\
\text { organization is useful in providing or collecting } \\
\text { information to ensure a comprehensive, consistent } \\
\text { and accurate response. other documentation is } \\
\text { needed to verify company compliance or to provide } \\
\text { input to mandated reports. }\end{array}$ \\
\hline
\end{tabular}




\begin{tabular}{|c|c|c|c|c|}
\hline $\begin{array}{l}\text { Risk Maragement, Assist top management in minimizing } \\
\text { Tisbitity by ident ifying risks and Implementing risk } \\
\text { management strategies. Identify potential for ICF KH } \\
\text { to adversely affect the environment or the publ ic, } \\
\text { damage corporate image, or otherwise be at risk due } \\
\text { actions related to enviromental protection. Take } \\
\text { appropriate measures to assist in the management of } \\
\text { this risk by identifying controls to minimize risk } \\
\text { and ensuring that comansurate benefits could result } \\
\text { from appropriate risk taking. (Note: this part of } \\
\text { the work scope is closely tied to all the others } \\
\text { because it is the other work scope functions that } \\
\text { allow this risk to be appropriately managed.) }\end{array}$ & 0.5 & 0 & 36.8 & $\begin{array}{l}\text { Significant civil and criminal liabilities may } \\
\text { result from violation of environmental laus and } \\
\text { regulations. These liabilities extend to } \\
\text { individuals and companies. An understanding of } \\
\text { the implications of noncompl lance is necessary to } \\
\text { ensure that the greatest complianee efforts are } \\
\text { dedicated to the obligations that have the } \\
\text { greatest likel ihood to be missed and the greatest } \\
\text { penal ty for noncompl lance. }\end{array}$ \\
\hline 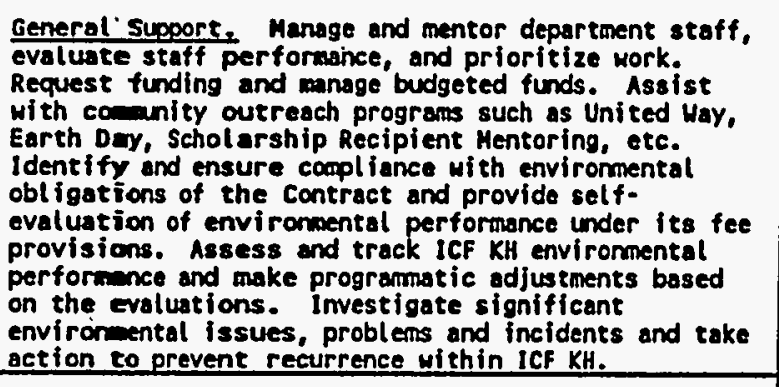 & 0.5 & & 36.8 & $\begin{array}{l}\text { This general support is necessary to ensure that } \\
\text { things are being done right and that the right } \\
\text { things are being done. }\end{array}$ \\
\hline Other cost Elements included: $(1,2,3)$ & & & 23.9 & $\dot{.}$ \\
\hline $\begin{array}{l}\text { Assessed Costs: These include Cost Elements } 4 \\
\text { (Services provided by UHC/ICFKH), } 5 \text { (Company level } \\
\text { services, ie: Dos imetry), } 6 \text { (IRH Services), and } 7 \\
\text { (DOH adders appl ied to Sugport from Others (abor). }\end{array}$ & & & 13.7 & - \\
\hline LEVEL I TOTALS & 7.0 & 0 & $\begin{array}{l}518.2 \\
\frac{13.7}{531.9}\end{array}$ & $\begin{array}{l}\text { Net Originated Cost } \\
\text { Assessed Cost } \\
\text { Total Budget (less direct labor of } \$ 20.3 \mathrm{~K} \text { ) }\end{array}$ \\
\hline
\end{tabular}




\begin{tabular}{|c|c|c|c|c|c|c|c|c|}
\hline \multicolumn{4}{|c|}{ Planned Staffing (Full Time Equivalent) } & \multicolumn{5}{|c|}{ Note: Job Family Only After 1997} \\
\hline Job Family $\%$ & Number: & अ४े & अ४। & अ, & अ४े। & ४४। & $14 \%$ & - \\
\hline Job Category & & 1996 & 1997 & $1998^{\circ}$ & 1999 & 2000 & 2001 & 2002 \\
\hline MANAGERS & अ४४ & ४ै। & ४४७ & ४ю & ४४ & ४ & ४ै। & थ। \\
\hline First line & 1 & 1 & 1 & & & & & \\
\hline \multicolumn{9}{|l|}{ General/executive } \\
\hline \multicolumn{9}{|l|}{ Projec/Program } \\
\hline \multicolumn{9}{|l|}{ Other } \\
\hline \multicolumn{9}{|l|}{ Subtotal } \\
\hline ENGINEERS & \%थ & ४० & ४ै। & 24 & $2 \%$ & $22_{2}$ & 2 & 2 \\
\hline \multicolumn{9}{|l|}{ Chemical } \\
\hline \multicolumn{9}{|l|}{ Civil } \\
\hline \multicolumn{9}{|l|}{ Computer } \\
\hline \multicolumn{9}{|l|}{ Electrical } \\
\hline Environmental & 2 & 2 & 2 & & & & & \\
\hline \multicolumn{9}{|l|}{ Industrial } \\
\hline \multicolumn{9}{|l|}{ Mechanical } \\
\hline \multicolumn{9}{|l|}{ Nuclear } \\
\hline \multicolumn{9}{|l|}{ Petroleum/Mining } \\
\hline \multicolumn{9}{|l|}{ Plant } \\
\hline \multicolumn{9}{|l|}{ Quality Control } \\
\hline \multicolumn{9}{|l|}{ Safety } \\
\hline \multicolumn{9}{|l|}{ Other } \\
\hline Subtotal & & & & & & & & \\
\hline
\end{tabular}




\begin{tabular}{|c|c|c|c|c|c|c|c|c|}
\hline \multicolumn{4}{|c|}{ Planned Staffing (Full Time Equivalent) } & \multicolumn{5}{|c|}{ Note: Job Family Only After 1997} \\
\hline Job Family & Number: & 3 & as & बे & अ. & अ? & अ: & נ। \\
\hline Job Category & & 1996 & 1997 & 1998 & 1999 & 2000 & 2001 & 2002 \\
\hline SCIENTISTS & मे. & \% & 3 & 3 & अ 3 & ?3: & 3 & 3 \\
\hline Chemists & & & & & & & & \\
\hline Environmental & 3 & 3 & 3 & & & & & \\
\hline Geologists & & & & & & & & \\
\hline Life & & & & & & & & \\
\hline Material & & & & & & & & \\
\hline Mathematicians & & & & & & & & \\
\hline Physicists & & & & & & & & \\
\hline Social & & & & & & & & \\
\hline Other & & & & & & & & \\
\hline Subtotal & & & & & & & & \\
\hline $\begin{array}{l}\text { ADMINISTRATIVE } / O T H E R \\
\text { PROFESSTONALS }\end{array}$ & अै०े & के & थै। & 1\%? & अ०ः & अै। & सा & 1 \\
\hline Accountant/auditor & & & & & & & . & \\
\hline Architect & & & & & & & & \\
\hline Buyers/Procurement & & & & & & & & \\
\hline Communications & & & & & & & & \\
\hline $\begin{array}{l}\text { Compliance } \\
\text { Inspectors }\end{array}$ & & & & . & & & & \\
\hline $\begin{array}{l}\text { Computer Sys } \\
\text { Analyst }\end{array}$ & & & & & & & & \\
\hline $\begin{array}{l}\text { Cost Est/p1 anner } \\
\text { /sch }\end{array}$ & & & & & & & & \\
\hline
\end{tabular}




\begin{tabular}{|c|c|c|c|c|c|c|c|c|}
\hline \multicolumn{4}{|c|}{ Planned Staffing (Full Time Equivalent) } & \multicolumn{5}{|c|}{ Note: Job Family Only After 1997} \\
\hline Job Famity & Number: & अి & ४४। & \& & की? & ४४। & 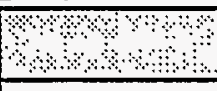 & बथ \\
\hline Job Category & & 1996 & 1997 & 1998 & 1999 & 2000 & 2001 & 2002 \\
\hline \multicolumn{9}{|l|}{ Health Physics } \\
\hline \multicolumn{9}{|l|}{ Industrial Hygiene } \\
\hline \multicolumn{9}{|l|}{ Lawyers } \\
\hline \multicolumn{9}{|l|}{$\begin{array}{l}\text { Personnel/1 abor } \\
\text { Relations }\end{array}$} \\
\hline \multicolumn{9}{|l|}{ Physicians } \\
\hline \multicolumn{9}{|l|}{$\begin{array}{l}\text { Physician } \\
\text { Assistant/Nurse }\end{array}$} \\
\hline $\begin{array}{l}\text { Safeguard \& } \\
\text { Security }\end{array}$ & 1 & 1 & 1 & & & & & \\
\hline \multicolumn{9}{|l|}{$\begin{array}{l}\text { Tech Writers \& } \\
\text { Editors }\end{array}$} \\
\hline \multicolumn{9}{|l|}{ Trainers } \\
\hline \multicolumn{9}{|l|}{ Other } \\
\hline \multicolumn{9}{|l|}{ Subtotal } \\
\hline $\begin{array}{l}\text { GENERAL } \\
\text { ADMIN/SECRETARY/CLERK }\end{array}$ & \%०ै & अे? & अथे & मिं & अै\% & अथे? & कि & s.......... \\
\hline \multicolumn{9}{|c|}{\begin{tabular}{|l|l|l|l|l|l|l|l|l|} 
Admin Assistant & & & & & & & & \\
\end{tabular}} \\
\hline \multicolumn{9}{|l|}{ Office Clerk (Gen) } \\
\hline \multicolumn{9}{|l|}{$\begin{array}{l}\text { Office Clerk } \\
\text { (Special) }\end{array}$} \\
\hline \multicolumn{9}{|l|}{ Secretaries } \\
\hline \multicolumn{9}{|l|}{$\begin{array}{l}\text { Typist/Word } \\
\text { Processor }\end{array}$} \\
\hline Other & & & & & & & & \\
\hline
\end{tabular}




\begin{tabular}{|c|c|c|c|c|c|c|c|c|}
\hline \multicolumn{4}{|c|}{ Planned Staffing (Full Time Equivalent) } & \multicolumn{5}{|c|}{ Note: Job Family Only After 1997} \\
\hline Job Family & Number: & $\therefore$ & + & $\mathrm{s}$ & WOOQ & अ & अ० & 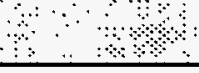 \\
\hline Job Category & & 1996 & 1997 & 1998 & 1999 & 2000 & 2001 & 2002 \\
\hline Subtotal & & & & & & & . & \\
\hline TECHNICIANS & में & 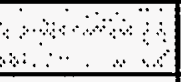 & ॥ै। & ख्य & eे & + & +s & \\
\hline $\begin{array}{l}\text { Computer } \\
\text { Operator/Coder }\end{array}$ & & & & & & & & \\
\hline Drafters & & & & & & & & \\
\hline Engineers/Technicians & & & & & & & & \\
\hline $\begin{array}{l}\text { Environmental } \\
\text { Science } \\
\text { Technicians }\end{array}$ & & & & & & & & - \\
\hline $\begin{array}{l}\text { Health Physics } \\
\text { Technicians } \\
\end{array}$ & & & & & & & & \\
\hline $\begin{array}{l}\text { Industrial } \\
\text { Saf/health } \\
\text { Technicians }\end{array}$ & & & & & & & & \\
\hline $\begin{array}{l}\text { Instru/Control } \\
\text { Technicians }\end{array}$ & & & & & & & & \\
\hline Lab. Technicians & & & & & & & & \\
\hline Media Technicians & & & & & & & & \\
\hline $\begin{array}{l}\text { Survey/Map } \\
\text { Technicians }\end{array}$ & & & & & & & & \\
\hline Other & & & & & & & & \\
\hline Subtotal & & & & & & & & \\
\hline 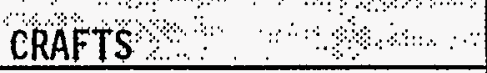 & ২े\% & \% & +े & $\%$ & । : & मे & बै & : \\
\hline Carpenters & & & & & & & & \\
\hline
\end{tabular}




\begin{tabular}{|c|c|c|c|c|c|c|c|c|}
\hline \multicolumn{4}{|c|}{ Planned Staffing (Full Time Equivalent) } & \multicolumn{5}{|c|}{ Note: Job Family Only After 1997} \\
\hline 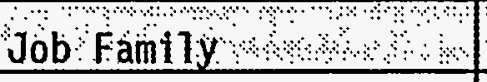 & Number: & अ० & \%०० & 4 & ৪ি & ४»ঃ & ४४। & अ \\
\hline Job Category & & 1996 & 1997 & 1998 & 1999 & 2000 & 2001 & 2002 \\
\hline Electricians & & & & & & . & & \\
\hline HVAC & & & & & & & & \\
\hline Machinists & & & & & & & & \\
\hline Masons & & & & & & & & \\
\hline Millwrights & & & & & & & & \\
\hline Painters & & & & & & & & \\
\hline Pimbrs/Pipefitters & & & & & & & & \\
\hline $\begin{array}{l}\text { Struct/Metal } \\
\text { Workers }\end{array}$ & & & & & & & & \\
\hline $\begin{array}{l}\text { Vehicl/Mobile } \\
\text { Equip. } \\
\text { Mechanics }\end{array}$ & & & & & & & & \\
\hline Welders & & & & & & & & \\
\hline other & & & & & & & & \\
\hline Subtotal & & & & & & & & \\
\hline OPERATORS & \%ै। & $2+\cdots$ & अै\% & कि & थे & अ? & \%ल & 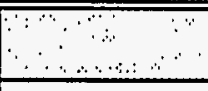 \\
\hline Lt. Vehicle Drivers & & & & & & & & \\
\hline $\begin{array}{l}\text { Material Moving } \\
\text { Equip. }\end{array}$ & & & & & & & & \\
\hline Nuiclear Plant & & & & & & & & \\
\hline $\begin{array}{l}\text { Utilities Waste } \\
\text { Proces }\end{array}$ & & & & & & & & \\
\hline Other & & & & & & & & \\
\hline Subtotal & & & & & & & & \\
\hline
\end{tabular}




\subsubsection{Industrial Safety and Health Services (WBS 6.5.1.7.2)}

\section{$\underline{\text { Mission }}$}

The ICF Kaiser Hanford Company (ICF KH) Industrial Safety and Health Services Department.(IS/H) interprets standards, develops procedures, establishes requirements, and provides company wide program direction, coordination, guidance and technical assistance to ensure the continued safety and health of employees.

\section{Products/Services Provided}

The Industrial Safety and Health Services Department provide the following services:

- Safety and Health: Provide a viable ICF KH Safety Program based on interpretation and applications of mandated requirements, orders, codes, standards and regulations. Implementation through ICF KH ESH Manual, WHC Manuals, and Field Safety Teams.

- Accident/Incident Records: Maintain OSHA (29 CFR 1904) statistics in accordance with DOE 5484.1, and performs tracking, trending, analysis, and evaluation of safety and health related "Case Management" data.

- Industrial Hygiene/Occ. Medical Records: Provide policies, programs, and procedural requirement as they relate. to Industrial Health (IH). Provide technical direction to $\mathrm{IH}$ field personnel to ensure interpretation and application consistency. Maintain interface with HEHF for employee medical records.

- Industrial Safety: Provide policies, programs and procedures as they relate to Industrial Safety (IS). Provide technical direction to field personnel to ensure interpretation and application consistency.

- Voluntary Protection Program (VPP): Mentor and facilitate the ICF KH Voluntary Protection Program and coordinate the Company Safety Improvement Program (SIP) and individual Division SIP's. Interface and bench mark with WHC VPP and other external VPP companies.

\section{Primary Customers}

The primary customer of the Industrial Safety and Health Services Department are: ICF Kaiser Hanford, Westinghouse Hanford Company, U.S. Department of Energy (RL). 
Craig Anderson Level I

Howard Budweg IS\&H Manager

\begin{tabular}{|c|c|c|c|c|}
\hline \multicolumn{5}{|l|}{ BUILDING BLOCKS FOR FY 1996} \\
\hline \multicolumn{5}{|l|}{ FY 1996 Building Blocks (\$000) } \\
\hline $\begin{array}{l}\text { ORGANIZATIOKAL HORK SCOPE DESCRIPTION } \\
\text { (bbbudweg - Part of IMKDAH) }\end{array}$ & $\begin{array}{l}\text { Org. FTEs } \\
\text { in } \\
\text { Horkscope }\end{array}$ & $\begin{array}{l}\text { Support } \\
\text { FTEs in } \\
\text { Horkscope }\end{array}$ & $\begin{array}{l}\text { FY } 1996 \\
\text { Originated } \\
\text { Cost* }\end{array}$ & $\begin{array}{c}\text { JUSTIFICATION OF SCOPE/IMPACT } \\
\text { IF HOT FUHDED }\end{array}$ \\
\hline $\begin{array}{l}\text { Safety and Heal th Services Dept.: } \\
\text { Progran/Policy and Achinistration }\end{array}$ & 3 & & 183.3 & $\begin{array}{l}\text { Responsible for viable ICFKH Safety Program based } \\
\text { on interpretation and appl ications of mandated } \\
\text { requirements, Orders, codes, Standards, and } \\
\text { Regulations. Program implementation through ICFKH } \\
\text { ESH Manual; intC Manuals, and Field Safety Teans. }\end{array}$ \\
\hline $\begin{array}{l}\text { Accident/Incident Records Group: } \\
\text { OSHA 200/Case Mgat/Safety Action Trending Systen }\end{array}$ & 2 & . & 73.3 & $\begin{array}{l}\text { Maintains OSHA (29CFR 1904) statistics in } \\
\text { accordance with DOE 5484.1, and performs } \\
\text { tracking, trending, analysis, and evaluation of } \\
\text { safety and heal th related "Case Management" data. } \\
\text { Mointain HEHF interface for case management and } \\
\text { interface with WHC Labor and Industries POC and } \\
\text { WHC Safety Awareness Dept. }\end{array}$ \\
\hline $\begin{array}{l}\text { Industrial Hygiene/Occupational Medical Records: } \\
\text { Programs/Procedures/Tech support/Rad. }\end{array}$ & 2 & & 147.0 & $\begin{array}{l}\text { Recommend, develop, and maintain policies, } \\
\text { prograns, and procectural requireants os they } \\
\text { relate to IH. Provide technical direction to it } \\
\text { field personel to ensure interpretation and } \\
\text { opplication consistency. Maintain Occ. Med } \\
\text { interface with HEHF for employee medical records. } \\
\text {. }\end{array}$ \\
\hline $\begin{array}{l}\text { Industrial Safety: } \\
\text { Prograns, Procedures/ Tech support } \\
\text { Support from Others }\end{array}$ & 2 & .5 & $\begin{array}{r}147.0 \\
47.0\end{array}$ & $\begin{array}{l}\text { Recormend, develop, and maintain policies, } \\
\text { prograns, and procedural requireaents as they } \\
\text { relate to Is. Provide technical direction to is } \\
\text { field personmel to ensure interpretation and } \\
\text { application consistency. }\end{array}$ \\
\hline $\begin{array}{l}\text { Voluntary Protection Program: } \\
\text { Facilitation company wide }\end{array}$ & 1 & & 73.5 & $\begin{array}{l}\text { Mentor and facillitate ICFKH Voluntary Protection } \\
\text { Program and coordinate Company Safety Improvement } \\
\text { PLan (SIP) and individual Division SIPs. } \\
\text { Interface and bench mark with WHC VPP and other } \\
\text { external VPP companies. }\end{array}$ \\
\hline Other cost Elements included: $(1,2,3)$ & & & 29.7 & \\
\hline
\end{tabular}


Craig Anderson Level I

Howard Budweg IS\&H Manager

\begin{tabular}{|c|c|c|c|c|}
\hline \multicolumn{5}{|l|}{ BUILDIKG BLOCKS FOR FY 1996} \\
\hline \multicolumn{5}{|l|}{ FY 1996 suilding Blocks (SO00) } \\
\hline $\begin{array}{l}\text { CRGANIZATIONAL WORK SCOPE DESCRIPTIOU } \\
\text { (bbbudweg - Part of IMKDAH) }\end{array}$ & $\begin{array}{l}\text { Org. FTEs } \\
\text { in } \\
\text { Workscope }\end{array}$ & $\begin{array}{l}\text { Support } \\
\text { FTEs in } \\
\text { Workscope }\end{array}$ & $\begin{array}{c}\text { FY } 1996 \\
\text { Originated } \\
\text { Cost* }\end{array}$ & $\begin{array}{l}\text { JUSTIFICATION OF SCOPE/IHPACT } \\
\text { If NOT FUNDED }\end{array}$ \\
\hline $\begin{array}{l}\text { Assessed Costs: These include Cost El ments } 4 \\
\text { (Services provided by wHC/ICFXH), } 5 \text { (company level } \\
\text { services, ie: Dosinetry), } 6 \text { (IRH Services), and } 7 \\
\text { (DOH adders applied to Support from Others labor). }\end{array}$ & & & 135.8 & \\
\hline LEVEL I TOTALS & 10 & .5 & $\begin{array}{l}680.8 \\
\frac{123.9}{804.7}\end{array}$ & $\begin{array}{l}\text { Net Originated Cost } \\
\text { Assessed Cost } \\
\text { Total Budget }\end{array}$ \\
\hline
\end{tabular}

* Originated cost $=$ Cost elements $0,1.2,3$ 


\begin{tabular}{|c|c|c|c|c|c|c|c|c|}
\hline \multicolumn{9}{|c|}{ Note: Job Family Only After 1997} \\
\hline Job Family & Nümber & 20 & $1 \% 3$ & ४े। & अ०४। & ४ै & ४ैै। & + \\
\hline Job Category & & 1996 & 1997 & 1998 & 1999 & 2000 & $200 \mathrm{i}^{\circ}$ & 2002 \\
\hline MANAGERS & +1? & m & ४ै। & 13 & $1 \%$ & अ $1 \%$ & अै। 1 । & 1 \\
\hline First line & 1 & 1 & 1 & & & & & \\
\hline \multicolumn{9}{|l|}{ General/executive } \\
\hline \multicolumn{9}{|l|}{ Projec/Program } \\
\hline \multicolumn{9}{|l|}{ other } \\
\hline \multicolumn{9}{|l|}{ Subtotal } \\
\hline ENGINEERS $\%$ \% & \%०। & \% & $2 \%$ & & $3 \%$ \% & $8.4 .4 \%$ & \%.।. & 4 \\
\hline \multicolumn{9}{|l|}{ Chemical } \\
\hline \multicolumn{9}{|l|}{ Civil } \\
\hline \multicolumn{9}{|l|}{ Computer } \\
\hline \multicolumn{9}{|l|}{ Electrical } \\
\hline \multicolumn{9}{|l|}{ Environmental } \\
\hline \multicolumn{9}{|l|}{ Industrial } \\
\hline \multicolumn{9}{|l|}{ Mechanical } \\
\hline \multicolumn{9}{|l|}{ Nuclear } \\
\hline \multicolumn{9}{|l|}{ Petroleum/Mining } \\
\hline \multicolumn{9}{|l|}{ Plant } \\
\hline \multicolumn{9}{|l|}{ Quality Control } \\
\hline Safety & 4 & 4 & 4 & & & & & \\
\hline \multicolumn{9}{|l|}{ Other } \\
\hline Subtotal & & & & & & & & \\
\hline
\end{tabular}




\begin{tabular}{|c|c|c|c|c|c|c|c|c|}
\hline \multicolumn{9}{|c|}{ Planned Staffing (Full Time Equivalent) } \\
\hline Job family y & Number: & 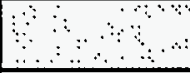 & ब & sथ & ब्थ & अ४े & $3+\%$ & \% \\
\hline Job Category & & 1996 & 1997 & 1998 & 1999 & 2000 & 2001 & 2002 \\
\hline SCIENTISTS & ४ै? & \% & बि & $2 \%$ & ४ै। & 2.2 & अ 2 & $2+3$ \\
\hline \multicolumn{9}{|l|}{ Chemists } \\
\hline Environmental & 2 & 2 & 2 & & & & & \\
\hline \multicolumn{9}{|l|}{ Geologists } \\
\hline \multicolumn{9}{|l|}{ Life } \\
\hline \multicolumn{9}{|l|}{ Material } \\
\hline \multicolumn{9}{|l|}{ Mathematicians } \\
\hline \multicolumn{9}{|l|}{ Physicists } \\
\hline \multicolumn{9}{|l|}{ Social } \\
\hline \multicolumn{9}{|l|}{ Other } \\
\hline \multicolumn{9}{|l|}{ Subtotal } \\
\hline $\begin{array}{l}\text { ADMINISTRATIVE/OTHER } \\
\text { PROFESSIONALS }\end{array}$ & अै। & 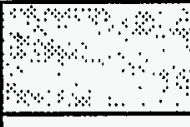 & ४ै। & अै। & ४ै: & अै। & मिन & as \\
\hline \multicolumn{9}{|c|}{\begin{tabular}{|l|l|l|l|l|l|l|} 
Accountant/auditor & & & & & & \\
\end{tabular}} \\
\hline \multicolumn{9}{|l|}{ Architect } \\
\hline \multicolumn{9}{|l|}{ Buyers/Procurement } \\
\hline \multicolumn{9}{|l|}{ Communications } \\
\hline \multicolumn{9}{|l|}{$\begin{array}{l}\text { Compliance } \\
\text { Inspectors }\end{array}$} \\
\hline \multicolumn{9}{|l|}{$\begin{array}{l}\text { Computer Sys } \\
\text { Analyst }\end{array}$} \\
\hline $\begin{array}{l}\text { Cost Est/planner } \\
\text { /sch }\end{array}$ & & & & & & & & \\
\hline
\end{tabular}




\begin{tabular}{|c|c|c|c|c|c|c|c|c|}
\hline \multicolumn{4}{|c|}{ Planned Staffing (Ful1 Time Equivalent) } & \multicolumn{5}{|c|}{ Note: Job Family Only After 1997} \\
\hline Job Family & Numbers & 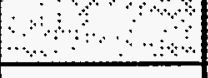 & + & \& & - & अन & में & $\therefore<$ \\
\hline Job Category & & 1996 & 1997 & 1998 & 1999 & 2000 & 2001 & 2002 \\
\hline Health Physics & & & & & & & & \\
\hline Industrial Hygiene & & . & & & & & & \\
\hline Lawyers & & & & & & & & \\
\hline $\begin{array}{l}\text { Personnel/labor } \\
\text { Relations }\end{array}$ & & & & & & & & \\
\hline Physicians & & & & & & & & \\
\hline $\begin{array}{l}\text { Physician } \\
\text { Assistant/Nurse }\end{array}$ & & & & & & & & \\
\hline $\begin{array}{l}\text { Safeguard \& } \\
\text { Security }\end{array}$ & & & & & & & & · \\
\hline $\begin{array}{l}\text { Tech Writers \& } \\
\text { Editors }\end{array}$ & & & & & & & & \\
\hline Trainers & & & & & & & & \\
\hline Other & & & & & & & & \\
\hline Subtotal & & & . & & & & & \\
\hline $\begin{array}{l}\text { GENERAL } \\
\text { ADMIN/SECRETARY/CLERK }\end{array}$ & क्ष & a & में & 33 & 3 & 3 & 3 & 3 \\
\hline Admin Assistant & & & & & & & & \\
\hline Office Clerk (Gen) & 2 & 2 & 2 & & & & & \\
\hline $\begin{array}{l}\text { Office Clerk } \\
\text { (Special) }\end{array}$ & & & & & & . & & \\
\hline Secretaries & 1 & 1 & 1 & & & & & \\
\hline $\begin{array}{l}\text { Typist/Word } \\
\text { Processor }\end{array}$ & & & & & & & & \\
\hline Other & & & & & & & & \\
\hline
\end{tabular}




\begin{tabular}{|c|c|c|c|c|c|c|c|c|}
\hline \multicolumn{4}{|c|}{ Planned Staffing (Full Time Equivalent) } & \multicolumn{5}{|c|}{ Note: Job Family Only After 1997} \\
\hline Job family & Numbers & मझ & ४ै। & \% & आ० & ४४४ & अ & «ি। \\
\hline Job Category & & 1996 & 1997 & 1998 & 1999 & 2000 & 2001 & 2002 \\
\hline Subtotal & & & & & & & & \\
\hline TECHNIGIANS & अ० & अथे & ४ै। & अ१ & अै। & अ०।: & अे।? & 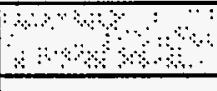 \\
\hline $\begin{array}{l}\text { Computer } \\
\text { Operator/Coder }\end{array}$ & & & & & & & & \\
\hline Drafters & & & & & & & & \\
\hline Engineers/Technicians & & & & & & & & \\
\hline $\begin{array}{l}\text { Environmental } \\
\text { Science } \\
\text { Technicians }\end{array}$ & & & & & & & & \\
\hline $\begin{array}{l}\text { Health Physics } \\
\text { Technicians }\end{array}$ & & & & & & & & \\
\hline $\begin{array}{l}\text { Industrial } \\
\text { Saf/health } \\
\text { Technicians }\end{array}$ & & & & & $\ddots$ & & & \\
\hline $\begin{array}{l}\text { Instru/Control } \\
\text { Technicians }\end{array}$ & & & & & & & & \\
\hline Lab. Technicians & & & & - & & & & \\
\hline Media Technicians & & & & & . & & & \\
\hline $\begin{array}{l}\text { Survey/Map } \\
\text { Technicians } \\
\end{array}$ & & . & & & & & & \\
\hline Other & & & & & & & & \\
\hline Subtotal & & & & & & & & \\
\hline CRAFTS & १०० & ४\% & $2 \%$ & œ४: & ४४० & ४े. & अ, : & \\
\hline Carpenters & & & & & & & & \\
\hline
\end{tabular}

言 


\begin{tabular}{|c|c|c|c|c|c|c|c|c|}
\hline \multicolumn{4}{|c|}{ Planned Staffing (Full Time Equivalent) } & \multicolumn{5}{|c|}{ Note: Job Family Only After 1997} \\
\hline Job familiy & Number: & $4+$ & 4 & 80.8 & अ४ & ए४⿻ & \%०० & \%० \\
\hline Job Category & & 1996 & 1997 & 1998 & 1999 & 2000 & 2001 & 2002 \\
\hline Electricians & & & & & & & & \\
\hline HVAC & & & & & & & & \\
\hline Machinists & & & & & & & & \\
\hline Masons & & & & & & & & \\
\hline Millwrights & & & & & & & & \\
\hline Painters & & & & & & & & \\
\hline Pimbrs/Pipefitters & & & & & & & & \\
\hline $\begin{array}{l}\text { Struct/Metal } \\
\text { Workers }\end{array}$ & & & & & & & & \\
\hline $\begin{array}{l}\text { Vehicl/Mobile } \\
\text { Equip. } \\
\text { Mechanics }\end{array}$ & & & & & & & & \\
\hline Welders & & & & & & & & \\
\hline other & & & & & & & - & \\
\hline Subtotal & & & & & & & & \\
\hline OPERATORS & \%. & 4 & 20 & 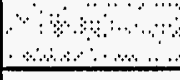 & men & पि? & Wan. & \\
\hline Lt. Vehicle Drivers & & & & & & & & \\
\hline $\begin{array}{l}\text { Material Moving } \\
\text { Equip. }\end{array}$ & & & & & & & & \\
\hline Nuclear $\mathrm{Plant}$ & & & & & & & & \\
\hline $\begin{array}{l}\text { Utilities Waste } \\
\text { Proces }\end{array}$ & & & & & & & & \\
\hline other & & & & & & & & \\
\hline Subtotal & & & & & & & & \\
\hline
\end{tabular}




\begin{tabular}{|c|c|c|c|c|c|c|c|c|}
\hline \multicolumn{4}{|c|}{ Planned Staffing (Full Time Equivalent) } & \multicolumn{5}{|c|}{ Note: Job Family Only After 1997} \\
\hline Job Famfly & Number: & $u_{2}$ & is & क्ष & ए। & \%े & ४ै। & 3 \\
\hline Job Category & & 1996 & 1997 & 1998 & 1999 & 2000 & 2001 & 2002 \\
\hline $\begin{array}{l}\text { ABOR } \& \text { GENERAL } \\
\text { WORKERS }\end{array}$ & +3 & +2. & अेंद & & 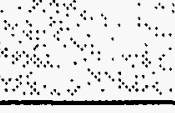 & ४ै। & +s: & \\
\hline Firefighters & & & & & & & & \\
\hline Food Service & & & & & & & & \\
\hline Hand/Help Lab Gen & & & & & & & & \\
\hline $\begin{array}{l}\text { Hand/Help Lab } \\
\text { Special }\end{array}$ & & & & & & & & \\
\hline Janitors/Cleaners & & & & & & & & \\
\hline Laundry Workers & & & & & & & & \\
\hline Security Guards & & & & & & & & \\
\hline Other & & & & & & & & \\
\hline Subtotal & & & & & & & & \\
\hline TOTAL FTES & & & & & & & & \\
\hline
\end{tabular}




\subsubsection{Procedures and Training (WBS 6.5.1.7.3)}

\section{$\underline{\text { Mission }}$}

The mission of the Central Support Services organization which includes procedures and training is to provide the technical and administrative services needed by ICF KH personnel.

\section{Products/Services Provided}

The Central Support Services Organization provides the following services:

- Procedures: For company procedures, maintain program, edit procedures, distribute procedures for review and approval, maintain controlled distribution, train points of contact and conduct self-assessments.

- Training: Maintain company wide training program, point of contact with WHC and HAMMER training, maintain training tracking system (EMPORTS), chair company training council.

\section{Primary Customer}

The primary customers of the Central Support Services organization are: ICF Kaiser Hanford, and Westinghouse Hanford Company. 
PROCEDURES/TRAINING PROGRAM

\begin{tabular}{|c|c|c|c|c|}
\hline $\begin{array}{l}\text { ORGANIZATIOHAL NORK SCOPE DESCRIPTION } \\
\text { (budal - Part of MMKDAH) }\end{array}$ & $\begin{array}{l}\text { ORG. FTES IN } \\
\text { HORKSCOPE }\end{array}$ & $\begin{array}{l}\text { SUPPORT FTES } \\
\text { IN HORKSCOPE }\end{array}$ & $\begin{array}{l}\text { FY } 1996 \\
\text { ORIGINATED } \\
\text { COST* }\end{array}$ & $\begin{array}{l}\text { JUSTIFICATION OF SCOPE/IHPACT IF NOT } \\
\text { FUNDED }\end{array}$ \\
\hline $\begin{array}{l}\text { Procedures (MKD03 - CC } 57750 \text { ) For } \\
\text { company procedures, instructions and } \\
\text { controlled forms: Maintain program; } \\
\text { edit procedures; distribute procedures } \\
\text { for review and approval; maintain } \\
\text { controlled distribution; train points of } \\
\text { contact, conduct self-assessments. } \\
\text { Provide graphics design services to the } \\
\text { company for Value Engineering Studies, } \\
\text { presentations, procedures, uncontrolled } \\
\text { forms, and company-sensitive documents } \\
\text { (eg org, charts, business plans \& } \\
\text { strategies) } \\
\text { Support from others } \\
\text { other Cost Elements included: }(1,2.3)\end{array}$ & 4 & 0 & \begin{tabular}{|l}
$\$ 214,200$ \\
$\$ 32,100$ \\
$\$ 60,400$ \\
$\$ 3,400$ \\
\end{tabular} & $\begin{array}{l}\text { Will not comply with 10CFR830.120, } \\
\text { DOE } 5700.6 C \text { and ICF KH Quality } \\
\text { Management Plan. Will lose cost } \\
\text { efficiencies due to standard } \\
\text { operating procedures. } \\
\text { Needed for company wide support for } \\
\text { Risk: graphics-reiated tasks. Work } \\
\text { is required and would need to be } \\
\text { funded from another source. }\end{array}$ \\
\hline $\begin{array}{l}\text { Training Program (MKOET - CC 57700) } \\
\text { Maintain company wide training program, } \\
\text { point of contact with WHC and HAMHER } \\
\text { training, maintain training tracking } \\
\text { system (EMPORTS), chair company training } \\
\text { council. } \\
\text { Other Cost Elements included: }(1,2.3)\end{array}$ & 0.7 & & $\begin{array}{l}\$ 43,500 \\
\$ 3.800 \\
\end{array}$ & $\begin{array}{l}\text { Will not comply with 10CFR830.120, } \\
\text { DOE 5700.6C and ICF KH Quality } \\
\text { Management Plan. Will lose cost } \\
\text { efficiencies due to standard } \\
\text { operating procedures. }\end{array}$ \\
\hline $\begin{array}{l}\text { Assessed Costs: These include Cost } \\
\text { Elements } 4 \text { (Services provided by } \\
\text { WHC/ICFKH), } 5 \text { (Company level services, } \\
\text { ie: Dosimetry), } 6 \text { (IRM Services), and } 7 \\
\text { (DOH adders applied to Support from } \\
\text { Others labor). }\end{array}$ & & & 539,400 & . \\
\hline LEVEL 'I'TOTALS & 5.7 & 1.8 & $\begin{array}{l}\$ 357,400 \\
\$ 39,400 \\
\$ 396.800 \\
\end{array}$ & $\begin{array}{l}\text { Net Originated Cost } \\
\text { Assessed Cost } \\
\text { Total Budget }\end{array}$ \\
\hline
\end{tabular}




\begin{tabular}{|c|c|c|c|c|c|c|c|c|}
\hline \multicolumn{4}{|c|}{ Planned Staffing (Full Time Equivalent) } & \multicolumn{5}{|c|}{ Note: Job Family Only After 1997} \\
\hline Job famtiy $\mathrm{s}$ & Number & 3 & अै। & अ०? & ४०० & कीजि: & ४४य & कि। \\
\hline Job Category & & 1996 & 1997 & 1998 & 1999 & 2000 & 2001 & 2002 \\
\hline MANAGERS & \%०\% & ४ & & थ, ? & थै। & ४थ & \%०, & 2 \\
\hline \multicolumn{9}{|l|}{ First line } \\
\hline \multicolumn{9}{|l|}{ General/executive } \\
\hline \multicolumn{9}{|l|}{ Projec/Program } \\
\hline \multicolumn{9}{|l|}{ Other } \\
\hline \multicolumn{9}{|l|}{ Subtotal } \\
\hline ENGINEERS & \%? & खे & सेखि & एक⿻ & थि & \%ण, & किल & \\
\hline \multicolumn{9}{|l|}{ Chemical } \\
\hline \multicolumn{9}{|l|}{ Civil } \\
\hline \multicolumn{9}{|l|}{ Computer } \\
\hline \multicolumn{9}{|l|}{ Electrical } \\
\hline \multicolumn{9}{|l|}{ Environmental } \\
\hline \multicolumn{9}{|l|}{ Industrial } \\
\hline \multicolumn{9}{|l|}{ Mechanical } \\
\hline \multicolumn{9}{|l|}{ Nuclear } \\
\hline \multicolumn{9}{|l|}{ Petroleum/Mining } \\
\hline \multicolumn{9}{|l|}{ Plant } \\
\hline \multicolumn{9}{|l|}{ Quality Control } \\
\hline \multicolumn{9}{|l|}{ Safety } \\
\hline \multicolumn{9}{|l|}{ other } \\
\hline Subtotal & & & & & & & & \\
\hline
\end{tabular}




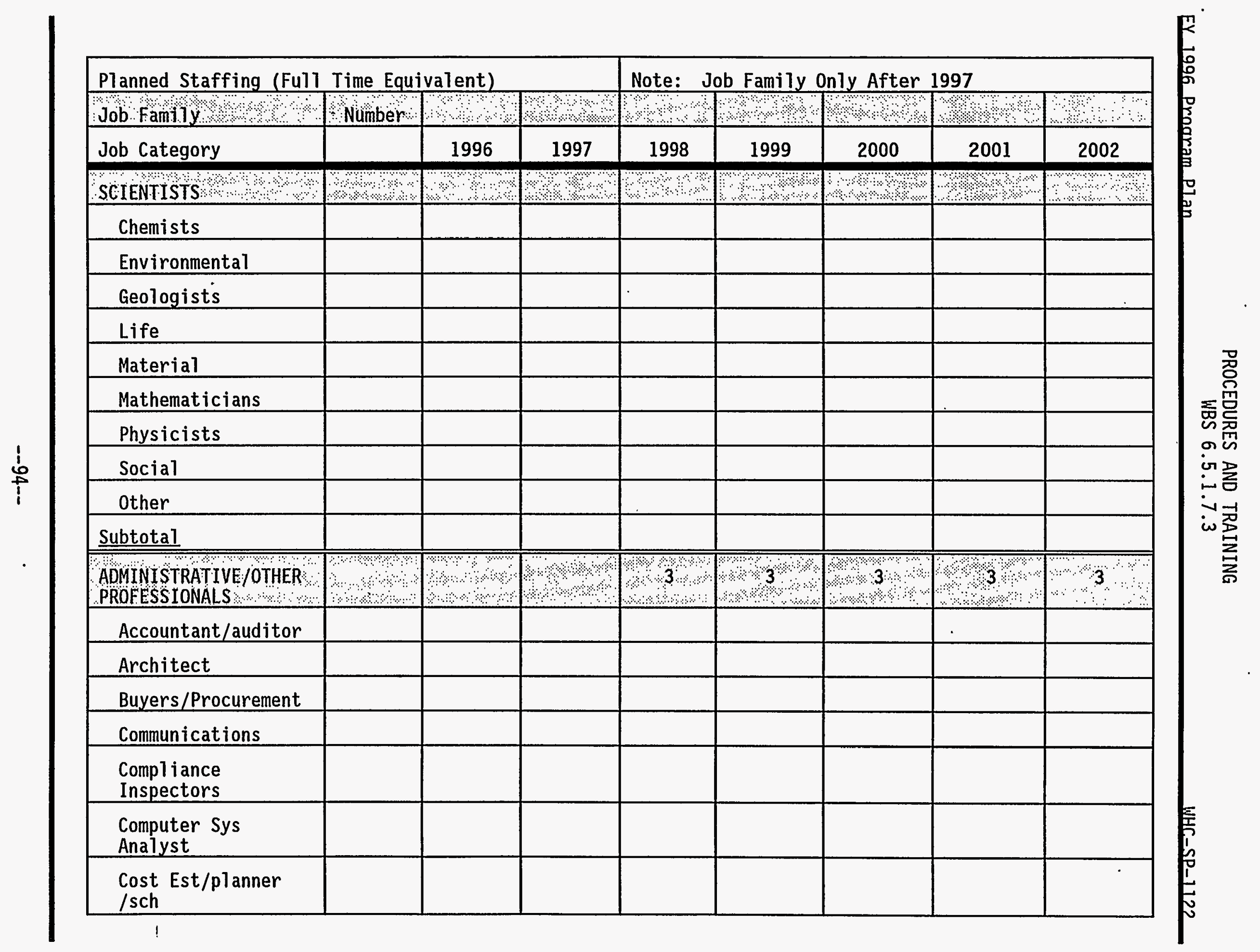




\begin{tabular}{|c|c|c|c|c|c|c|c|c|}
\hline \multicolumn{9}{|c|}{ Planned Staffing (Full Time Equivalent) } \\
\hline Job Famity $\mathrm{s}$ & Number & : : & \%० & 8 & अें & ४४। & $2+\cdots$ & 2 \\
\hline Job Category & & 1996 & 1997 & 1998 & 1999 & 2000 & 2001 & 2002 \\
\hline \multicolumn{9}{|l|}{ Health Physics } \\
\hline \multicolumn{9}{|l|}{ Industrial Hygiene } \\
\hline \multicolumn{9}{|l|}{ Lawyers } \\
\hline \multicolumn{9}{|l|}{$\begin{array}{l}\text { Personnel/labor } \\
\text { Relations }\end{array}$} \\
\hline \multicolumn{9}{|l|}{ Physicians } \\
\hline \multicolumn{9}{|l|}{$\begin{array}{l}\text { Physician } \\
\text { Assistant/Nurse }\end{array}$} \\
\hline \multicolumn{9}{|l|}{$\begin{array}{l}\text { Safeguard \& } \\
\text { Security }\end{array}$} \\
\hline $\begin{array}{l}\text { Tech Writers \& } \\
\text { Editors }\end{array}$ & 1 & 1 & 1 & & & & & \\
\hline Trainers & 1 & 1 & 1 & & & & & \\
\hline Other & 1 & 1 & 1 & & & & & \\
\hline \multicolumn{9}{|l|}{ Subtotal } \\
\hline $\begin{array}{l}\text { GENERAL } \\
\text { ADMIN/SECRETARY /CLERK }\end{array}$ & भै०े? & $\%$ & अथे & अ? & (3. & \% & 3 & 3 \\
\hline \multicolumn{9}{|l|}{ Admin Assistant } \\
\hline Office Clerk (Gen) & 3 & 3 & 3 & & & & & \\
\hline \multicolumn{9}{|l|}{$\begin{array}{l}\text { Office Clerk } \\
\text { (Special) }\end{array}$} \\
\hline \multicolumn{9}{|l|}{ Secretaries } \\
\hline \multicolumn{9}{|l|}{$\begin{array}{l}\text { Typist/Word } \\
\text { Processor }\end{array}$} \\
\hline Other & & & & & & & & \\
\hline
\end{tabular}




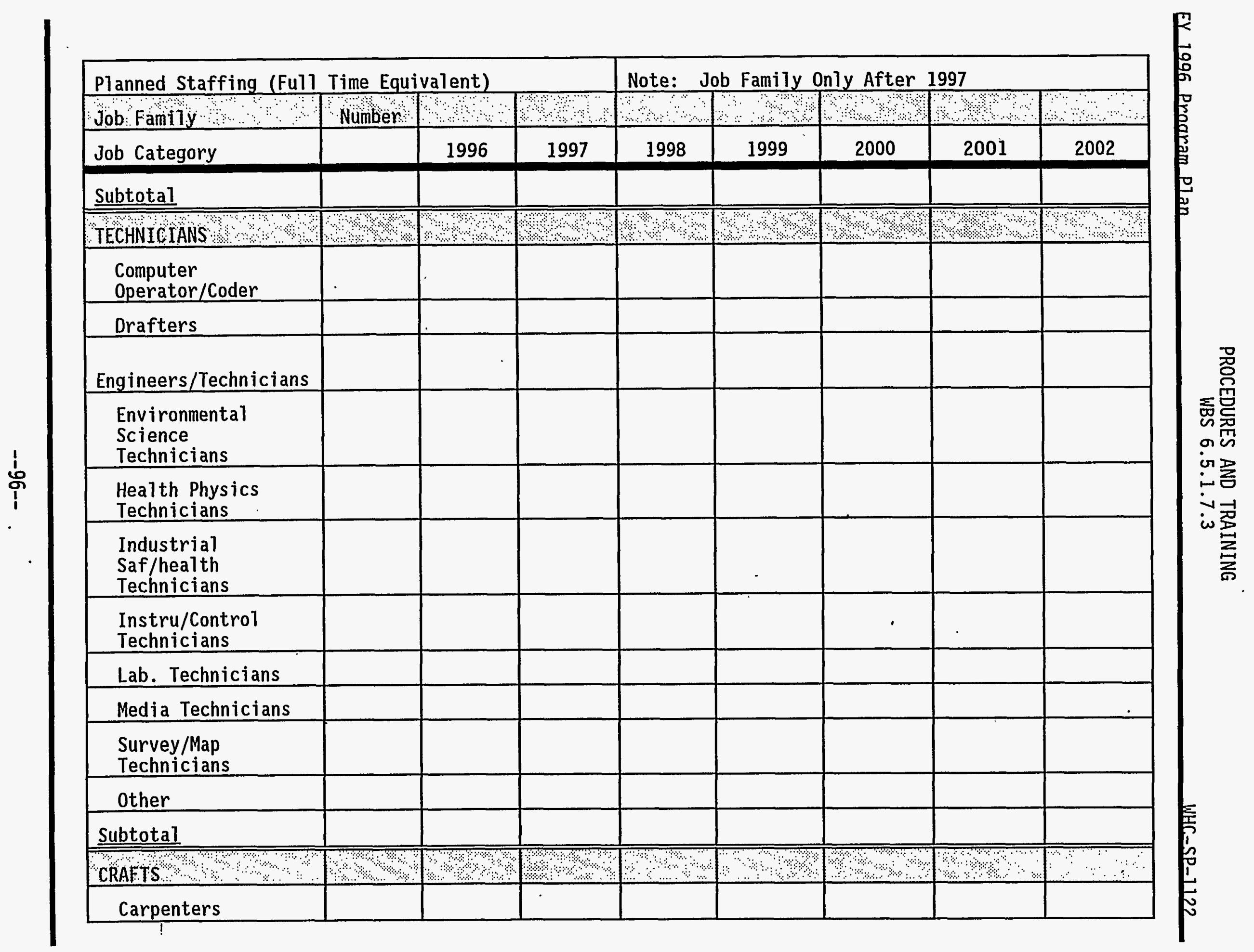




\begin{tabular}{|c|c|c|c|c|c|c|c|c|}
\hline \multicolumn{4}{|c|}{ Planned Staffing (Full Time Equivalent) } & \multicolumn{5}{|c|}{ Note: Job Family Only After 1997} \\
\hline Job family $\quad$ ४४ & Number: & +. & MQ\% & 3 & थै & घho. & $+\%$ & mo \\
\hline Job Category & & 1996 & 1997. & 1998 & 1999 & 2000 & 2001 & 2002 \\
\hline \multicolumn{9}{|l|}{ Electricians } \\
\hline \multicolumn{9}{|l|}{ HVAC } \\
\hline \multicolumn{9}{|l|}{ Machinists } \\
\hline \multicolumn{9}{|l|}{ Masons } \\
\hline \multicolumn{9}{|l|}{ Millwrights } \\
\hline \multicolumn{9}{|l|}{ Painters } \\
\hline \multicolumn{9}{|l|}{ P1mbrs/Pipefitters } \\
\hline \multicolumn{9}{|l|}{$\begin{array}{l}\text { Struct/Metal } \\
\text { Workers }\end{array}$} \\
\hline \multicolumn{9}{|l|}{$\begin{array}{l}\text { Vehicl/Mobile } \\
\text { Equip. } \\
\text { Mechanics }\end{array}$} \\
\hline \multicolumn{9}{|l|}{ Welders } \\
\hline \multicolumn{9}{|l|}{ other } \\
\hline \multicolumn{9}{|l|}{ Subtotal } \\
\hline \multicolumn{9}{|c|}{ 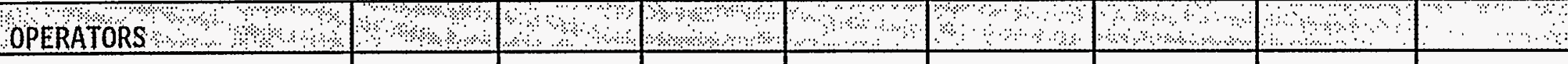 } \\
\hline \multicolumn{9}{|l|}{ Lt. Vehicle Drivers } \\
\hline \multicolumn{9}{|l|}{$\begin{array}{l}\text { Material Moving } \\
\text { Equip. }\end{array}$} \\
\hline \multicolumn{9}{|l|}{ Nuclear Plant } \\
\hline \multicolumn{9}{|l|}{$\begin{array}{l}\text { Utilities Waste } \\
\text { Proces }\end{array}$} \\
\hline \multicolumn{9}{|l|}{\begin{tabular}{|l|} 
other \\
\end{tabular}} \\
\hline Subtotal & & & & & & & & \\
\hline
\end{tabular}




\begin{tabular}{|c|c|c|c|c|c|c|c|c|}
\hline \multicolumn{4}{|c|}{ Planned Staffing (Full Time Equivalent) } & \multicolumn{5}{|c|}{ Note: Job Family Only After 1997} \\
\hline Job Family & Number & 2 & एँ? & 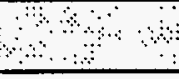 & +4) & $10 \times$ & थै। & and \\
\hline Job Category & & 1996 & 1997 & 1998 & 1999 & 2000 & 2001 & 2002 \\
\hline $\begin{array}{l}\text { CABOR \& GENERAL } \\
\text { WORKERS }\end{array}$ & ?+4 & Qै। & \%? & r. & अै? & $2+$ & Yै? & \\
\hline Firefighters & & & & & & & & \\
\hline Food Service & & & & & & & & \\
\hline Hand/Help Lab Gen & & & & & & & & \\
\hline $\begin{array}{l}\text { Hand/Help Lab } \\
\text { Special }\end{array}$ & & & & & & & & \\
\hline Janitors/Cleaners & & & & & & & & \\
\hline Laundry Workers & & & & & & & & \\
\hline Security Guards & & & & & & & & \\
\hline Other & & & & & & & & \\
\hline Subtotal & & & & & & & & \\
\hline TOTAL FTES & & & & & & & & \\
\hline
\end{tabular}




\subsubsection{Environmental, Safety, Health and Quality Performance Assessment (WBS 6.5.1.8)}

\section{$\underline{\text { Mission }}$}

The mission of ESH\&Q PA organization is to provide to the executive management and general manager's office documented determination of managerial effectiveness in establishing and implementing effective program plans that conform to DOE and WHC requirements in an efficient, timely and cost conscious manner.

\section{Products/Services Provided}

The ESH\&Q PA organization provide the following services:

- Management Assessment: Co-ordinate, conduct planned, periodic, and integrated Management Assessment, in accordance with the Quality Management Program. Perform assessment/investigations as required by senior management.

- Compliance Assessment: Perform internal audits and surveillance for Environmental, Quality, and Safety and Health Programs as identified on the audit and surveillance schedule; administer the Risk Evaluation system.

- Corrective Action Management/Analysis: Maintain the interface with the Hanford Action Tracking System (HATS) database; maintain and generate the monthly Corrective Action Status Tracking and Trending Report.

\section{Primary Customers}

The primary customers of ESH\&Q PA are: ICF Kaiser Hanford, Westinghouse Hanford Company, and U.S. Department of Energy (RL). 


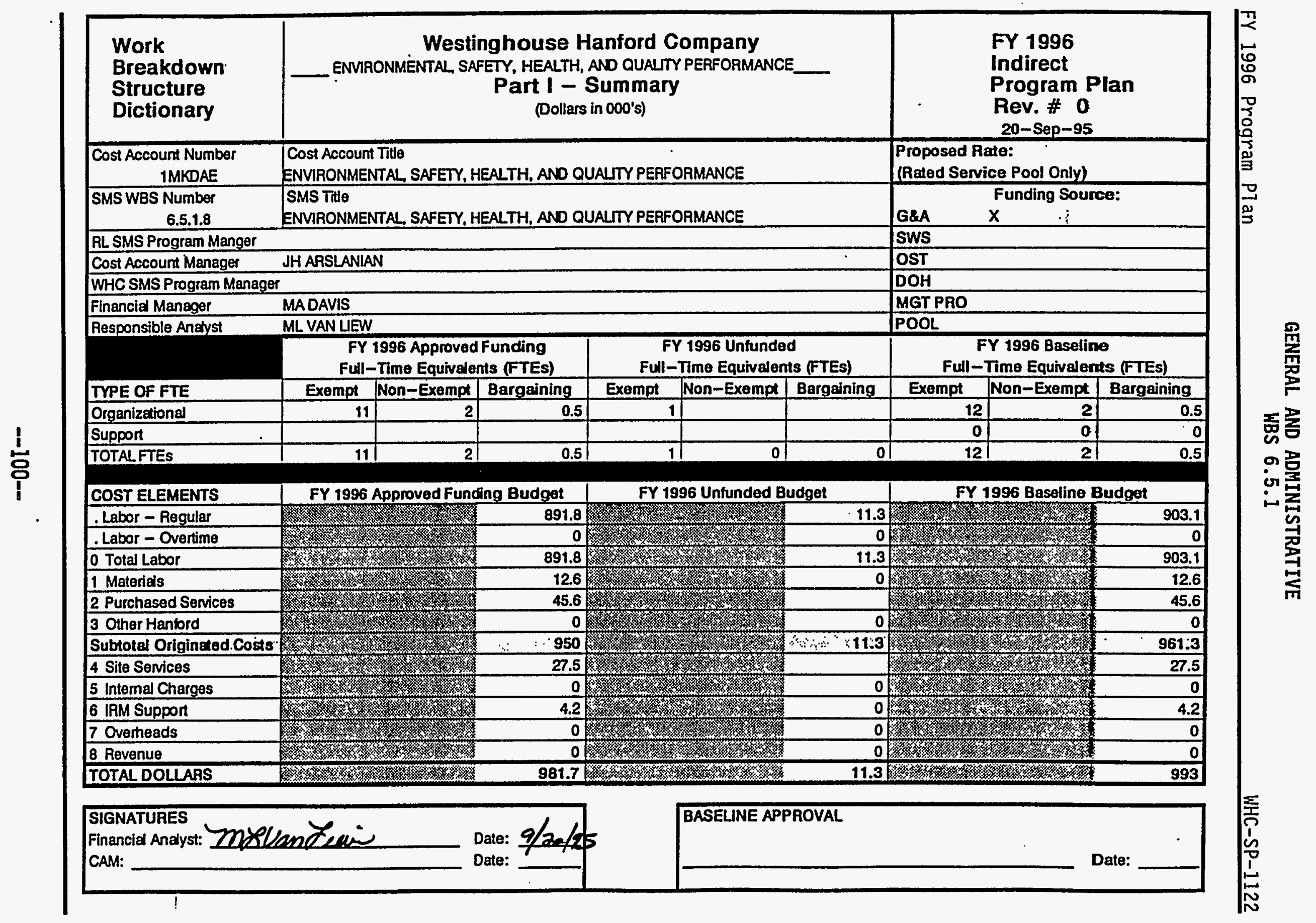




\section{ESH\&Q PERFORMANCE ASSESSMENT DIVISION \\ ORGANIZATION CODE \#50050 \\ FY 1996 BUILDING BLOCKS}

\section{REVISED TO INCLUDE UNFUNDED WORK ON PAGE 3}

\begin{tabular}{|c|c|c|c|c|}
\hline $\begin{array}{l}\text { ORGAHIZATIONAL MORK SCOPE DESCRIPTIOH } \\
\text { (bldgblk.rV1 - 1MKDAE) }\end{array}$ & $\begin{array}{l}\text { Org. FTEs } \\
\text { in } \\
\text { Workscope }\end{array}$ & $\begin{array}{l}\text { Support } \\
\text { FTEs in } \\
\text { Horkscope }\end{array}$ & $\begin{array}{c}\text { FY } 1996 \\
\text { Originated Cost }\end{array}$ & JUSTIFICATION OF SCOPE/IMPACT IF NOT FUNDED \\
\hline $\begin{array}{l}\text { Managenent Assessment Activities include: } \\
\text { conduct planned, periodic, and integrated } \\
\text { Management Assessments in accordance with } \\
\text { the Qual ity Management Program; generate. } \\
\text { reports; recommend Best Management Practices } \\
\text { to improve the quality of the program } \\
\text { assessed; provide object ive, timely, and } \\
\text { reliable information on performance, } \\
\text { including significant achievements related } \\
\text { to the scope of the assessment; follow-up on } \\
\text { the effectiveness and promptness of actions } \\
\text { taken by the responsible managers in } \\
\text { resolving assessment concerns; and respond } \\
\text { to requests for special assessments from the } \\
\text { General Manager/Deputy General Manager. }\end{array}$ & 3 & & $\$ 180,600$ & $\begin{array}{l}10 \text { CFR } 830.112 \text { "Unreviewed Safety Questions" } \\
10 \text { CFR } 830.120 \text { "Quality Assurance" } \\
10 \text { CFR } 830.320 \text { "Technical Safety Requirements" } \\
10 \text { CFR } 830.330 \text { "Training and Certification" } \\
\text { DOE Order } 5700.6 C \\
\text { ICF KH Quality Management Program } \\
\text { ASME NQA-1 Muclear Quality Assurance Program } \\
\text { ICF KH Procedure "Management Assess- } \\
\text { ment/Appraisal Program" } \\
\text { IMPACT - Violation of above requirements if } \\
\text { WOrksCope Mas not performed/funded including } \\
\text { Price Anderson Ammencment Act (PAAA) } \\
\text { implications. }\end{array}$ \\
\hline
\end{tabular}




\begin{tabular}{|c|c|c|c|c|}
\hline $\begin{array}{l}\text { ORGAHI2ATIOHAL WORK SCOPE DESCRIPTION } \\
\text { (bldgblk. rVI - IMKDAE) }\end{array}$ & $\begin{array}{l}\text { Org. FTEs } \\
\text { in } \\
\text { Horkscope }\end{array}$ & $\begin{array}{l}\text { Support } \\
\text { FTES in } \\
\text { Workscope }\end{array}$ & $\begin{array}{l}\text { FY } 1996 \\
\text { Originated Cost }\end{array}$ & JUSTIFICATION CF SCOPE/IMPACT IF NOT FUNDED \\
\hline $\begin{array}{l}\text { Compl iance Assessment Activities } \\
\text { (audit/surveillance activities) include: } \\
\text { pertorm internal audits and surveillances } \\
\text { for Environmental, Quality, and Safety and } \\
\text { Heal th Programs as identified on the Audit } \\
\text { and Surveillance schedule; administer the } \\
\text { Risk Evaluation system; monitor activities } \\
\text { for significant adverse conditions to } \\
\text { determine the need for issuance of } \\
\text { Corrective Action Requests; and conduct } \\
\text { follow-ups on corrective action comnitments } \\
\text { for internal cARs, audits, and surveillances } \\
\text { for closure. }\end{array}$ & 9 & & $\$ 661,600$ & $\begin{array}{l}10 \text { CFR } 830.112 \text { "UnrevieHed Safety Questions" } \\
10 \text { CFR } 830.120 \text { "Quality Assurance" } \\
10 \text { CFR } 830.320 \text { "Technical Safety Requirements" } \\
10 \text { CFR } 830.330 \text { "Training and Certification" } \\
\text { DOE Order } 5700.6 C \\
\text { ICF KH Quality Management Program } \\
\text { ASME NOA-1 Muclear Quality Assurance Program } \\
\text { IMPACT - Violation of above requirements if } \\
\text { POrkscope was not performed/funded including } \\
\text { implice Anderson Ammenctionst Act (PAAA) }\end{array}$ \\
\hline $\begin{array}{l}\text { Corrective Action Management/Analysis } \\
\text { Activities include: maintain the interface } \\
\text { with the Hanford Action Tracking System } \\
\text { (HATS) database; maintain and generate the } \\
\text { monthly Corrective Action Status Tracking } \\
\text { Report as a means for tracking of corrective } \\
\text { actlon cormi tments which includes } \\
\text { maintenance of the HATS database; maintain } \\
\text { and generate the monthly Trend Analysis } \\
\text { Report and revise the report to include key } \\
\text { performance indicators as they are } \\
\text { ldentified. }\end{array}$ & 1.5 & 0 & $\$ 49,600$ & $\begin{array}{l}10 \text { CFR } 830.120 \text { "Quality Assurance" } \\
\text { DOE Order } 5700.6 C \\
\text { ICF KH Qual ity Management Program } \\
\text { IHPACT - Violation of above requirements if } \\
\text { Norkscope HBS not performed/funded including } \\
\text { Price Anderson Armenchent ACt (PAAA) } \\
\text { impl ications. }\end{array}$ \\
\hline Other Cost Elements included: $(1,2,3)$. & & & $\$ 58,200$ & \\
\hline $\begin{array}{l}\text { Assessed Costs: These include Cost Elements } \\
4 \text { (services provided by HHC/ICFKH), } 5 \\
\text { (Company level services, ie: Dosimetry), } 6 \\
\text { (IRM Services), and } 7 \text { (DoH adders applied to } \\
\text { support from others (abor). }\end{array}$ & & & $\$ 31,700$ & . \\
\hline
\end{tabular}




\begin{tabular}{|c|c|c|c|c|}
\hline $\begin{array}{l}\text { ORGANIZATIONAL HORK SCOPE DESCRIPTION } \\
\text { (bldgblK.rVI - 1MKDAE) }\end{array}$ & $\begin{array}{l}\text { Org. FTES } \\
\text { in } \\
\text { Horkscope }\end{array}$ & $\begin{array}{l}\text { Suppoirt } \\
\text { FTES in } \\
\text { Horkscope } \\
\end{array}$ & $\begin{array}{c}\text { FY } 1996 \\
\text { Originated Cost }\end{array}$ & JUSTIFICATION OF SCOPE/IHPACT IF NOT FUNDED \\
\hline LEVEL I TOTALS & $\begin{array}{c}13.5 \\
\vdots\end{array}$ & : & $\begin{array}{l}\$ 950,000 \\
\$ 31,700 \\
\$ \$ 981,700\end{array}$ & $\begin{array}{l}\text { Net Originated Cost/Target: } \$ 950 \mathrm{~K} \\
\text { Assessed Costs } \\
\text { Total Budget } \\
\text { *BUDGET DOLLARS CUT TO } \$ 950,000 \\
\text { FROM A SUBMITTAL OF } \$ 1,144,390 \\
\end{array}$ \\
\hline 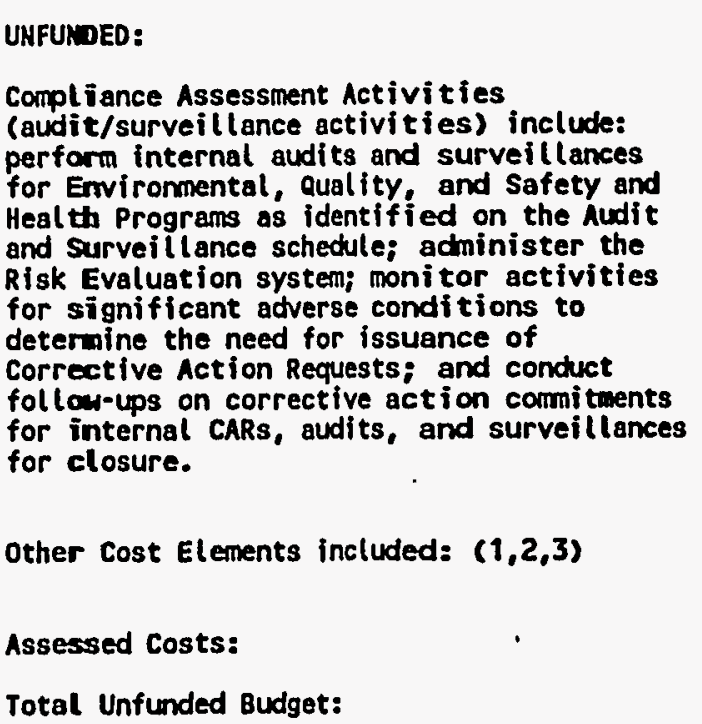 & $1 *$ & $i$ & $\begin{array}{l}\$ 73,500 \\
\vdots \\
\$ 60,000 \\
\frac{\$ 8,900}{\$ 142,400}\end{array}$ & $\begin{array}{l}10 \text { CFR } 830.112 \text { "Unreviewed Safety Questions" } \\
10 \text { CFR } 830.120 \text { "Quality Assurance" } \\
10 \text { CFR } 830.320 \text { "Technical Safety Requirements" } \\
\text { 10 CFR } 830.330 \text { "Training and Certification" } \\
\text { DOE Order } 5700.6 C \\
\text { ICF KH Quality Management Program } \\
\text { ASME NQA-1 Huclear Qual ity Assurance Program } \\
\text { IMPACT - Violation of above requirements if } \\
\text { Horkscope was not performed/funded including } \\
\text { Price Anderson Ammencment Act (PAMA) } \\
\text { implications. } \\
\text { "1 FTE added in workscope due to loss of } 3 \\
\text { consul tants that cannot be funded during FY } \\
\text { 1996. }\end{array}$ \\
\hline
\end{tabular}




\begin{tabular}{|c|c|c|c|c|c|c|c|c|}
\hline \multicolumn{4}{|c|}{ Planned Staffing (Full Time Equivalent) } & \multirow{2}{*}{\multicolumn{5}{|c|}{ 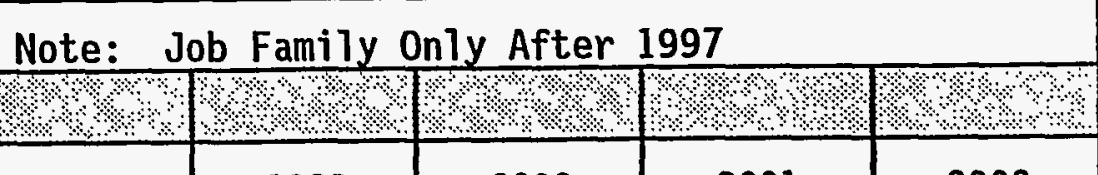 }} \\
\hline Sob family 4 & Number: & 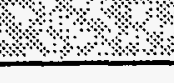 & (४): & & & & & \\
\hline Job Category & & 1996 & 1997 & 1998 & 1999 & 2000 & 2001 & \\
\hline MANAGERS & 2.2 & 4.8 & 4089 & 2.8 & 2.2 .8 & 282.2 & 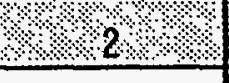 & 102, \\
\hline First line & 1 & 1 & 1 & & & & & \\
\hline General/executive & 1 & 1 & 1 & & & & & \\
\hline Projec/Program & & & & & & & & \\
\hline Other & & & & & & & & \\
\hline Subtotal & & & & & & & & \\
\hline ENGINEERS: & 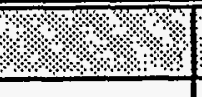 & 1.28 & 18 & $.5 \%$ & $8.8 \%$ & $4.8 \%$ & $8.5 \%$ & 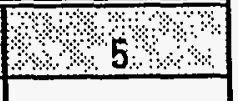 \\
\hline Chemical & & & & & & & & \\
\hline Civil & & & & & & & & \\
\hline Computer & & & & & & & & \\
\hline Electrical & & & & & & & & \\
\hline Environmental & & & & & & & & \\
\hline Industrial & & & & & & & & \\
\hline Mechanical & & & & & & & & \\
\hline Nuclear & & & & & & & & \\
\hline Petroleum/Mining & & & & & & & & \\
\hline Plant & $\underline{2}$ & 2 & $\underline{2}$ & . & & & & \\
\hline Quality Control & & & & & & & & \\
\hline Safety & 3 & 3 & 3 & & & & & \\
\hline Other & & & & & & & & \\
\hline Subtotal & & & & & & & & \\
\hline
\end{tabular}




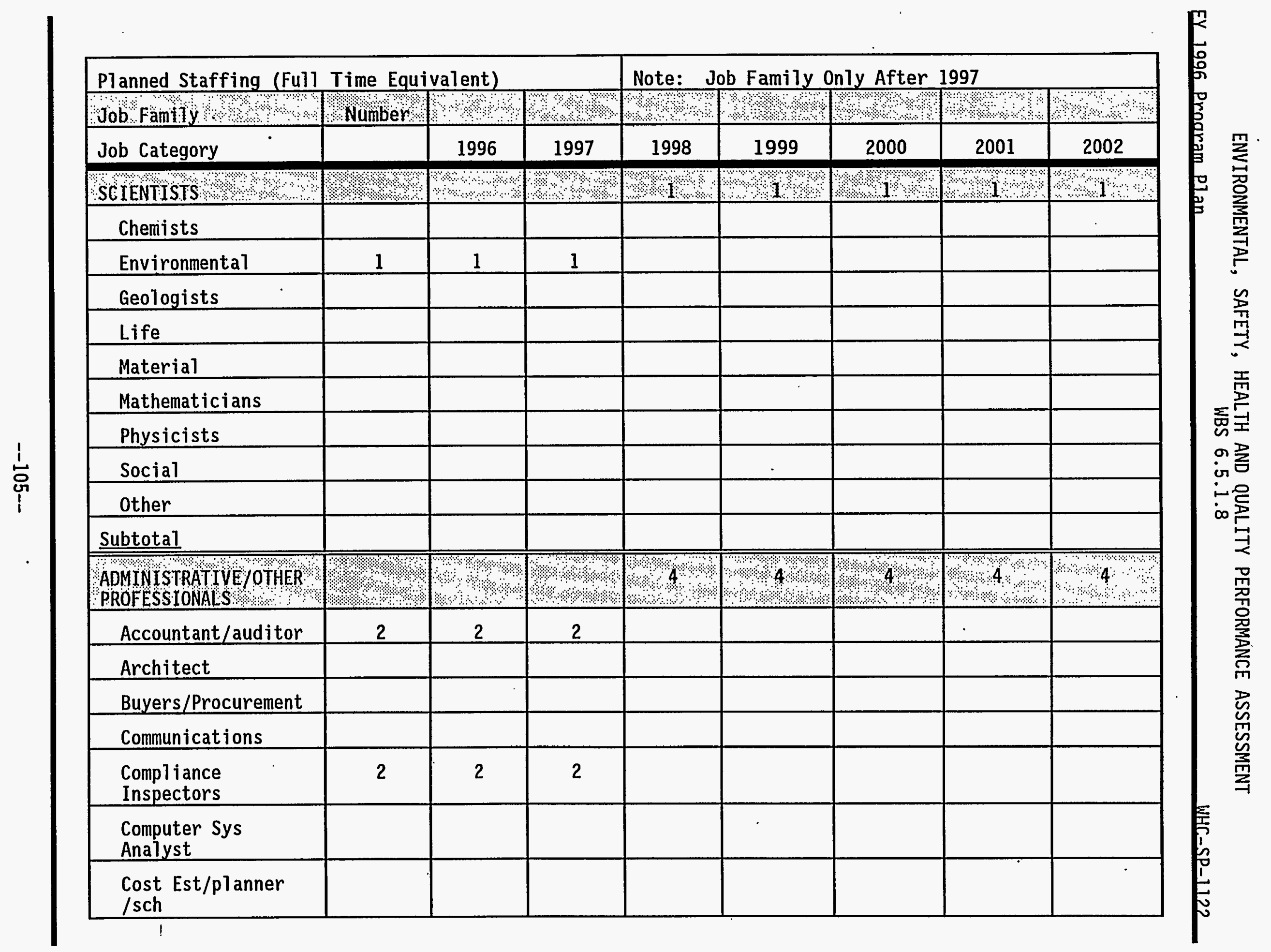




\begin{tabular}{|c|c|c|c|c|c|c|c|c|}
\hline \multicolumn{4}{|c|}{ Pl anned Staffing (Full Time Equivalent) } & \multicolumn{5}{|c|}{ Note: Job Family Only After 1997} \\
\hline Job family & Number: & औरे०? & $1 \%$ & ४४ & अै? & ४४०४४ & \%थ & \\
\hline Job Category & & 1996 & 1997 & 1998 & 1999 & 2000 & 2001 & 2002 \\
\hline \multicolumn{9}{|l|}{ Health Physics } \\
\hline \multicolumn{9}{|l|}{ Industrial Hygiene } \\
\hline \multicolumn{9}{|l|}{ Lawyers } \\
\hline \multicolumn{9}{|l|}{$\begin{array}{l}\text { Personnel/1abor } \\
\text { Relations }\end{array}$} \\
\hline \multicolumn{9}{|l|}{ Physicians } \\
\hline \multicolumn{9}{|l|}{$\begin{array}{l}\text { Physician } \\
\text { Assistant/Nurse }\end{array}$} \\
\hline \multicolumn{9}{|l|}{$\begin{array}{l}\text { Safeguard \& } \\
\text { Security }\end{array}$} \\
\hline \multicolumn{9}{|l|}{$\begin{array}{l}\text { Tech Writers \& } \\
\text { Editors }\end{array}$} \\
\hline \multicolumn{9}{|l|}{ Trainers } \\
\hline \multicolumn{9}{|l|}{ other } \\
\hline \multicolumn{9}{|l|}{ Subtotal } \\
\hline $\begin{array}{l}\text { GENERAL } \\
\text { AOMIN/SECRETARY/CLERR }\end{array}$ & 2.2? & $2^{2}$ & 18 & 1.5 & 2.5 & 14.5 & . 1.5 & 1.5 \\
\hline \multicolumn{9}{|l|}{ Admin Assistant } \\
\hline Office Clerk (Gen) & 0.5 & 0.5 & 0.5 & & & & & \\
\hline \multicolumn{9}{|l|}{$\begin{array}{l}\text { Office Clerk } \\
\text { (Special) }\end{array}$} \\
\hline Secretaries & 1 & 1 & 1 & & & & & \\
\hline \multicolumn{9}{|l|}{$\begin{array}{l}\text { Typist/Word } \\
\text { Processor }\end{array}$} \\
\hline other & & & & & & & & \\
\hline
\end{tabular}




\begin{tabular}{|c|c|c|c|c|c|c|c|c|}
\hline \multicolumn{4}{|c|}{ Planned Staffing (Full Time Equivalent) } & \multicolumn{5}{|c|}{ Note: Job Family Only After 1997} \\
\hline Job Family $y$ & Number: & 4 & अ? & Qै? & 14\% & ॠै। & $3 \%$ & \%० \\
\hline Job Category & & 1996 & 1997 & 1998 & 1999 & 2000 & 2001 & 2002 \\
\hline \multicolumn{9}{|l|}{ Subtotal } \\
\hline TECHNICIANS & -2:2: & $4 \times 8$ & +2 & 11 & अ1\% & 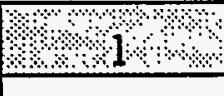 & $8 \times 1 \%$ & 11 \\
\hline \multicolumn{9}{|l|}{$\begin{array}{l}\text { Computer } \\
\text { Operator/Coder }\end{array}$} \\
\hline \multicolumn{9}{|l|}{ Drafters } \\
\hline Engineers/Technicians & 1 & 1 & 1 & & & & & \\
\hline \multicolumn{9}{|l|}{$\begin{array}{l}\text { Environmental } \\
\text { Science } \\
\text { Technicians } \\
\end{array}$} \\
\hline \multicolumn{9}{|l|}{$\begin{array}{l}\text { Health Physics } \\
\text { Technicians }\end{array}$} \\
\hline \multicolumn{9}{|l|}{$\begin{array}{l}\text { Industrial } \\
\text { Saf/health } \\
\text { Technicians }\end{array}$} \\
\hline \multicolumn{9}{|l|}{$\begin{array}{l}\text { Instru/Control } \\
\text { Technicians }\end{array}$} \\
\hline \multicolumn{9}{|l|}{ Lab. Technicians } \\
\hline \multicolumn{9}{|l|}{ Media Technicians } \\
\hline \multicolumn{9}{|l|}{$\begin{array}{l}\text { Survey/Map } \\
\text { Technicians }\end{array}$} \\
\hline \multicolumn{9}{|l|}{ other } \\
\hline \multicolumn{9}{|l|}{ Subtotal } \\
\hline GRAFTS & $2=2$ & $\%$ & $12+2$ & \%? & +\% & 1:. & $8 \times$ & \\
\hline \multicolumn{9}{|l|}{ Carpenters } \\
\hline Electricians & & & & & & & & \\
\hline
\end{tabular}




\begin{tabular}{|c|c|c|c|c|c|c|c|c|}
\hline \multicolumn{9}{|c|}{ Note: Job Family Only After 1997} \\
\hline 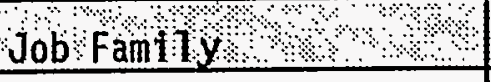 & Number: & मे। & ४ै० & ४े? & ४० & $8 \%$ & \%थ\% & ४४४ \\
\hline Job Category & & 1996 & 1997 & 1998 & 1999 & 2000 & 2001 & 2002 \\
\hline \multicolumn{9}{|l|}{ HVAC } \\
\hline \multicolumn{9}{|l|}{ Machinists } \\
\hline \multicolumn{9}{|l|}{ Masons } \\
\hline \multicolumn{9}{|l|}{ Millwrights } \\
\hline \multicolumn{9}{|l|}{ Painters } \\
\hline \multicolumn{9}{|l|}{ Plmbrs/Pipefitters } \\
\hline \multicolumn{9}{|l|}{$\begin{array}{l}\text { Struct/Metal } \\
\text { Workers }\end{array}$} \\
\hline \multicolumn{9}{|l|}{$\begin{array}{l}\text { Vehicl/Mobile } \\
\text { Equip. } \\
\text { Mechanics }\end{array}$} \\
\hline \multicolumn{9}{|l|}{ Welders } \\
\hline \multicolumn{9}{|l|}{ Other } \\
\hline \multicolumn{9}{|l|}{ Subtotal } \\
\hline OPERATORS $\%$ & \% \% & $\%$ & 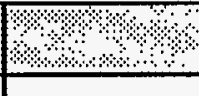 & १०० & अ०००: & \%०\%? & अ०० & \%े \\
\hline \multicolumn{9}{|l|}{ Lt. Vehicle Drivers } \\
\hline \multicolumn{9}{|l|}{$\begin{array}{l}\text { Material Moving } \\
\text { Equip. }\end{array}$} \\
\hline \multicolumn{9}{|l|}{ Nuclear Plant } \\
\hline \multicolumn{9}{|l|}{$\begin{array}{l}\text { Utilities'Waste } \\
\text { Proces }\end{array}$} \\
\hline \multicolumn{9}{|l|}{ Other } \\
\hline Subtotal & & & $=$ & & & & & \\
\hline
\end{tabular}




\begin{tabular}{|c|c|c|c|c|c|c|c|c|}
\hline \multicolumn{4}{|c|}{ Planned Staffing (Full Time Equivalent) } & \multicolumn{5}{|c|}{ Note: Job Family Only After 1997} \\
\hline Job Famtiy & Number: & men & +: & 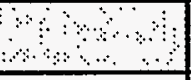 & +4 & ४ै४४४। & ४म, & का" \\
\hline Job Category & & 1996 & 1997 & 1998 & 1999 & 2000 & 2001 & 2002 \\
\hline $\begin{array}{l}\text { ABOR \& GENERAL } \\
\text { WORKERS }\end{array}$ & थे? & $8+$ & 2 & \%४ & अ? & 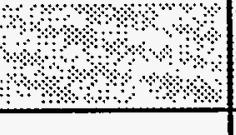 & ?े। & थै। \\
\hline \multicolumn{9}{|l|}{ Firefighters } \\
\hline \multicolumn{9}{|l|}{ Food Service } \\
\hline \multicolumn{9}{|l|}{ Hand/Help Lab Gen } \\
\hline \multicolumn{9}{|l|}{$\begin{array}{l}\text { Hand/Help Lab } \\
\text { Special }\end{array}$} \\
\hline \multicolumn{9}{|l|}{ Janitors/Cleaners } \\
\hline \multicolumn{9}{|l|}{ Laundry Workers } \\
\hline \multicolumn{9}{|l|}{ Security Guards } \\
\hline \multicolumn{9}{|l|}{ other } \\
\hline \multicolumn{9}{|l|}{ Subtotal } \\
\hline TOTAL FTES & & & & & & & & \\
\hline
\end{tabular}




\subsubsection{Labor Relations (WBS 6.5.1.9)}

Mission.

The mission of the Labor Relations Department is to provide a full range of labor relations services and represent the company in collective bargaining relationships with HAMTC, OPEIU, the Building and Construction Trades, QC/NDT, and the Escorts.

\section{Product/Services Provided}

The Labor Relations organization performs the following:

- HAMTC Labor Relations: Administer and interpret agreement, resolve disputes and

grievances, advise management, and administer policies.

- Building Trades Labor Relations: Administer and interpret agreement, resolve disputes and grievances, advise management, and administer policies.

- Clerical Support: Process hires and terminations, records maintenance, medical scheduling, etc.

- Labor Relations Management: Negotiate five (5) different collective bargaining agreements, resolve disputes and grievances, establish policy, and advise company management.

- $\quad$ Represents the company in arbitrations and NLRB procedures.

\section{Primary Customers}

The primary customers of Labor Relations are: ICF Kaiser Hanford, Labor Unions and U.S. Department of Energy (RL). 


\begin{tabular}{|c|c|c|c|c|c|c|c|c|}
\hline $\begin{array}{l}\text { Work } \\
\text { Breakdown } \\
\text { Structure } \\
\text { Dictionary }\end{array}$ & \multicolumn{5}{|c|}{$\begin{array}{c}\text { Westinghouse Hanford Company } \\
\text { LABOR RELATIONS } \\
\text { Part 1 - Summary } \\
\text { (Dollars in 000's) }\end{array}$} & \multicolumn{3}{|c|}{$\begin{array}{l}\text { FY } 1996 \\
\text { Indirect } \\
\text { Program Plan } \\
\text { Rev. \# } 0 \\
\text { 24-Aug-95 }\end{array}$} \\
\hline $\begin{array}{c}\text { Cost Account Number } \\
\text { 1MKDAL }\end{array}$ & \multicolumn{5}{|l|}{$\begin{array}{l}\text { Cost Account Title } \\
\text { LABOR RELATIONS }\end{array}$} & \multicolumn{3}{|c|}{$\begin{array}{l}\text { Proposed Rate: } \\
\text { (Rated Service Pool Only) }\end{array}$} \\
\hline $\begin{array}{r}\text { SMS WBS Number } \\
6.5 .1 .9 \\
\end{array}$ & \multicolumn{5}{|l|}{$\begin{array}{l}\text { SMS THIle } \\
\text { LABOR RELATIONS }\end{array}$} & \multicolumn{3}{|c|}{$\begin{array}{ll} & \text { Funding Source: } \\
\text { G\&A } & x^{-} \\
\end{array}$} \\
\hline RL SMS Program Manger & & & & & & \multicolumn{3}{|l|}{ Sws } \\
\hline Cost Account Manager & \multicolumn{5}{|l|}{ JA CARTER } & \multicolumn{3}{|l|}{ OST } \\
\hline WHC SMS Program Manager & & & & & & \multicolumn{3}{|l|}{$\mathrm{DOH}$} \\
\hline Financial Manager & \multicolumn{5}{|l|}{ MADAVIS } & \multicolumn{3}{|l|}{ MGT PRO } \\
\hline Responsible'Analyst & \multicolumn{5}{|l|}{ ML VAN LIEW } & \multicolumn{3}{|l|}{ POOL } \\
\hline & \multicolumn{2}{|c|}{$\begin{array}{l}\text { FY } 1996 \text { Approved Funding } \\
\text { Full-Time Equivalents (FTEs) }\end{array}$} & \multicolumn{3}{|c|}{$\begin{array}{l}\text { FY } 1996 \text { Unfunded } \\
\text { Full-Time Equivalents (FTEs) }\end{array}$} & \multicolumn{3}{|c|}{$\begin{array}{c}\text { FY } 1996 \text { Baseline } \\
\text { Full-Time Equivalents (FTEs) }\end{array}$} \\
\hline TYPE OF FTE & Exempt Non-Exempt & Bargaining & Exempt & Non-Exempt & Bargaining & Exempt & Non-Exempt & Bargaining \\
\hline Organizational & \begin{tabular}{|l|l|}
4 & 1 \\
\end{tabular} & \begin{tabular}{r|}
2 \\
\end{tabular} & & & & 4 & 1 & 2 \\
\hline Support & & 0.5 & & & & 0 & 0 & 0.5 \\
\hline TOTALFTES & 11 & 2.5 & 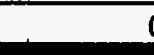 & 0 & & 4 & 1 & 2.5 \\
\hline COST ELEMENTS & FY 1996 Approved Fundi & ling Budget & FY 1 & 996 Unfunded Bc & udget & FY 1 & 1996 Baseline B & Sudget \\
\hline . Labor - Regular & 2 & 418.8 & 2 & 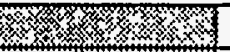 & & 13kn & 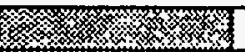 & 418.8 \\
\hline . Labor - Overtime & 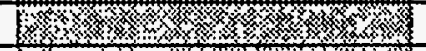 & 0 & 世. & r & & 10. & \% & 0 \\
\hline 0 Tolal Labor & S & 418.8 & 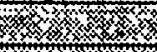 & (x) & & 0 & 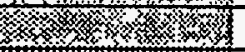 & 418.8 \\
\hline 1 Materials & 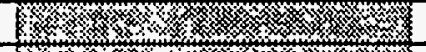 & 8.1 & z & 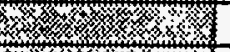 & & 7 & 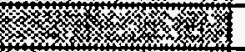 & 8.1 \\
\hline 2 Purchased Services & 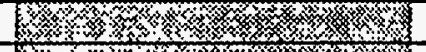 & 20.5 & Kro & 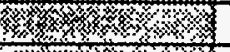 & & N. & 等 & 20.5 \\
\hline 3 Other Hantord & 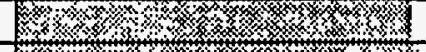 & & $x<x<$ & 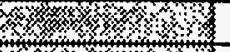 & & 18. & 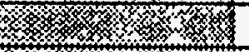 & 0 \\
\hline Subtotal Originated Costs & 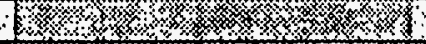 & 447.4 & 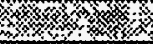 & $3 \times 3 \times 1 \times 1 \%>1$ & & $0 \%$ & 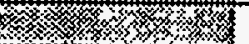 & 447.4 \\
\hline 4 Site Services & 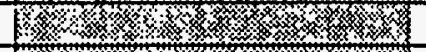 & 17.3 & W & $x$ x & & 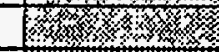 & 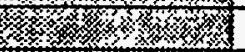 & 17.3 \\
\hline 5 Internal Charges & 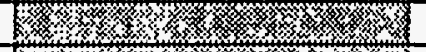 & & 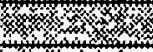 & 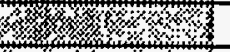 & & 隹 & 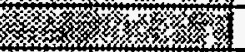 & 0 \\
\hline 6 IRM Support & 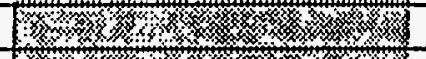 & 24 & $x$ & - & & $x_{2}$ & 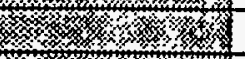 & 2.4 \\
\hline 7 Overheads & 16> & & 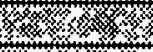 & 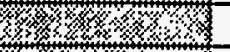 & & $y_{0}$ & 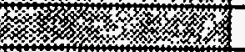 & 18 \\
\hline 8 Revenue & 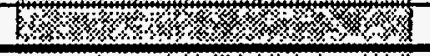 & & $3 x$ & $8626<080$ & & 28 & 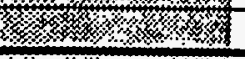 & 0 \\
\hline TOTAL DOLLARS & 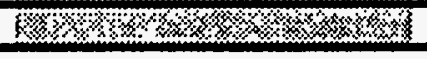 & 485.11 & $28 \times 8$ & $2 \times 8 \times 8$ & & 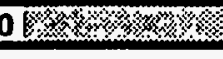 & 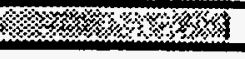 & 485.1 \\
\hline $\begin{array}{l}\text { SIGNATURES } \\
\text { Financial Analyst: Zuese } \\
\text { CAM: }\end{array}$ & Lvante & $\begin{array}{l}\text { Date: } 8 / 24 / 95 \\
\text { Date: }\end{array}$ & & BASELINE APP & PROVAL & & & )ate: \\
\hline
\end{tabular}




\begin{tabular}{|c|c|c|c|c|}
\hline BUILDING BLOCKS FOR FY 1996 & & & & \\
\hline \multicolumn{5}{|l|}{ FY 1996 Building Blocks ( 5000$)$} \\
\hline $\begin{array}{l}\text { ORGAMIZATIONAL WORK SCOPE DESCRIPTIOH } \\
\text { (FORH.MAD - IMKDAL) }\end{array}$ & $\begin{array}{l}\text { Org. FTEs } \\
\text { in } \\
\text { Horkscope }\end{array}$ & $\begin{array}{l}\text { Support } \\
\text { FTEs in } \\
\text { Uorkscope }\end{array}$ & $\begin{array}{l}\text { Fy } 1996 \\
\text { Originated } \\
\text { Cost* }\end{array}$ & $\begin{array}{l}\text { JUSTIFICATION OF SCOPE/IMPACT } \\
\text { IF NOT FUNDED }\end{array}$ \\
\hline $\begin{array}{l}\text { HAMTC Labor Relations } \\
\text { Administer Agreement } \\
\text { Interpret Agreement } \\
\text { Revolve Disputes } \\
\text { Resolve Grievances } \\
\text { Advise Hanagement } \\
\text { Administer Policies }\end{array}$ & $\begin{array}{l}2 \mathrm{EX} \\
.5 \mathrm{NEX}\end{array}$ & . & $\begin{array}{r}147.0 \\
16.8 \\
163.8\end{array}$ & $\begin{array}{l}\text { Service approximately } 800 \text { HAMTC employees } \\
\text { Expansion of grievances; loss of grievances } \\
\text { may/will incur: monetary damages; labor } \\
\text { disruptions; work stoppages; violations of } \\
\text { agreement will occur }\end{array}$ \\
\hline $\begin{array}{l}\text { Building Trades Labor Relations } \\
\text { Administer Agreement } \\
\text { Interpret Agreement } \\
\text { Resolve Disputes } \\
\text { Resolve Grievances } \\
\text { Advise Management } \\
\text { Administer Policies }\end{array}$ & q EX & & 73.5 & $\begin{array}{l}\text { Service approximately } 470 \text { Building Trades Craft } \\
\text { Expansion of grievances; loss of grievances } \\
\text { may/will incur: monetary damages; labor } \\
\text { disruptions; work stoppages; violations of } \\
\text { agreenent will occur }\end{array}$ \\
\hline $\begin{array}{l}\text { Clerical Support } \\
\text { Process Hires } \\
\text { Process Terminations } \\
\text { Records Maintenance } \\
\text { Medical Schedul ing } \\
\text { Compile Reports } \\
\text { Clerical Support } \\
\text { Support Subcontractors } \\
\end{array}$ & $2 \mathrm{Cl}$ & $\begin{array}{l}.5 \text { Craft- } \\
\text { Teanster }\end{array}$ & $\begin{array}{l}64.1 \\
27.0 \\
91.1\end{array}$ & $\begin{array}{l}\text { No ability to hire or terminate craft } \\
\text { Discontinue physicals } \\
\text { Violate labor agreement on manhauls } \\
\text {. }\end{array}$ \\
\hline $\begin{array}{l}\text { Labor Rel ations Management and Support } \\
\text { Negotiate Agreenents (5) } \\
\text { Resolve Grievances } \\
\text { Resolve Disputes } \\
\text { Establ ish Policy } \\
\text { Advise Managenent }\end{array}$ & $\begin{array}{l}1 \text { EX } \\
.5 \text { NEX }\end{array}$ & & $\begin{array}{l}73.5 \\
16.9 \\
90.4\end{array}$ & $\begin{array}{l}5 \text { collective bargaining agreements lapse. Wo } \\
\text { central company labor policy. Agreements are } \\
\text { violated. Increased arbitrations. Increase in } \\
\text { labor disputes. }\end{array}$ \\
\hline Other cost Elements included: $(1,2,3)$ & & & 28.6 & \\
\hline
\end{tabular}


LABOR RELATIONS

\begin{tabular}{|c|c|c|c|c|}
\hline BUILDING BLOCKS FOR FY 1996 & & & & \\
\hline FY 1996 Building Blocks (5000) & & & & \\
\hline $\begin{array}{l}\text { ORGAHIZATIONAL HORK SCOPE DESCRIPTION } \\
\text { (FORH.MAD - IMKDAL) }\end{array}$ & $\begin{array}{l}\text { Org. FTEs } \\
\text { in } \\
\text { Horkscope }\end{array}$ & $\begin{array}{l}\text { Support } \\
\text { FTEs in } \\
\text { Workscope }\end{array}$ & $\begin{array}{c}\text { FY } 1996 \\
\text { Originated } \\
\text { Cost* }\end{array}$ & $\begin{array}{l}\text { JUSTIFICATION OF SCOPE/IMPACT } \\
\text { IF NOT FUNDED }\end{array}$ \\
\hline $\begin{array}{l}\text { Assessed Costs: These include Cost Elements } 4 \\
\text { (Services provided by WHC/ICFKH), } 5 \text { (Company level } \\
\text { services, ie: Dosimetry), } 6 \text { (IRH Services), and } 7 \\
\text { (DOH adders appl ied to support from Others labor). }\end{array}$ & & & 37.7 & \\
\hline LEVEL 9 TOTALS & 7 & .5 & $\begin{array}{l}447.4 \\
37.7 \\
485.1\end{array}$ & $\begin{array}{l}\text { Net Originated Cost } \\
\text { Assessed Cost } \\
\text { Total Budget }\end{array}$ \\
\hline
\end{tabular}

$\stackrel{\omega}{\omega}$ Originated Cost $=$ Cost elements $0,1,2,3$ 


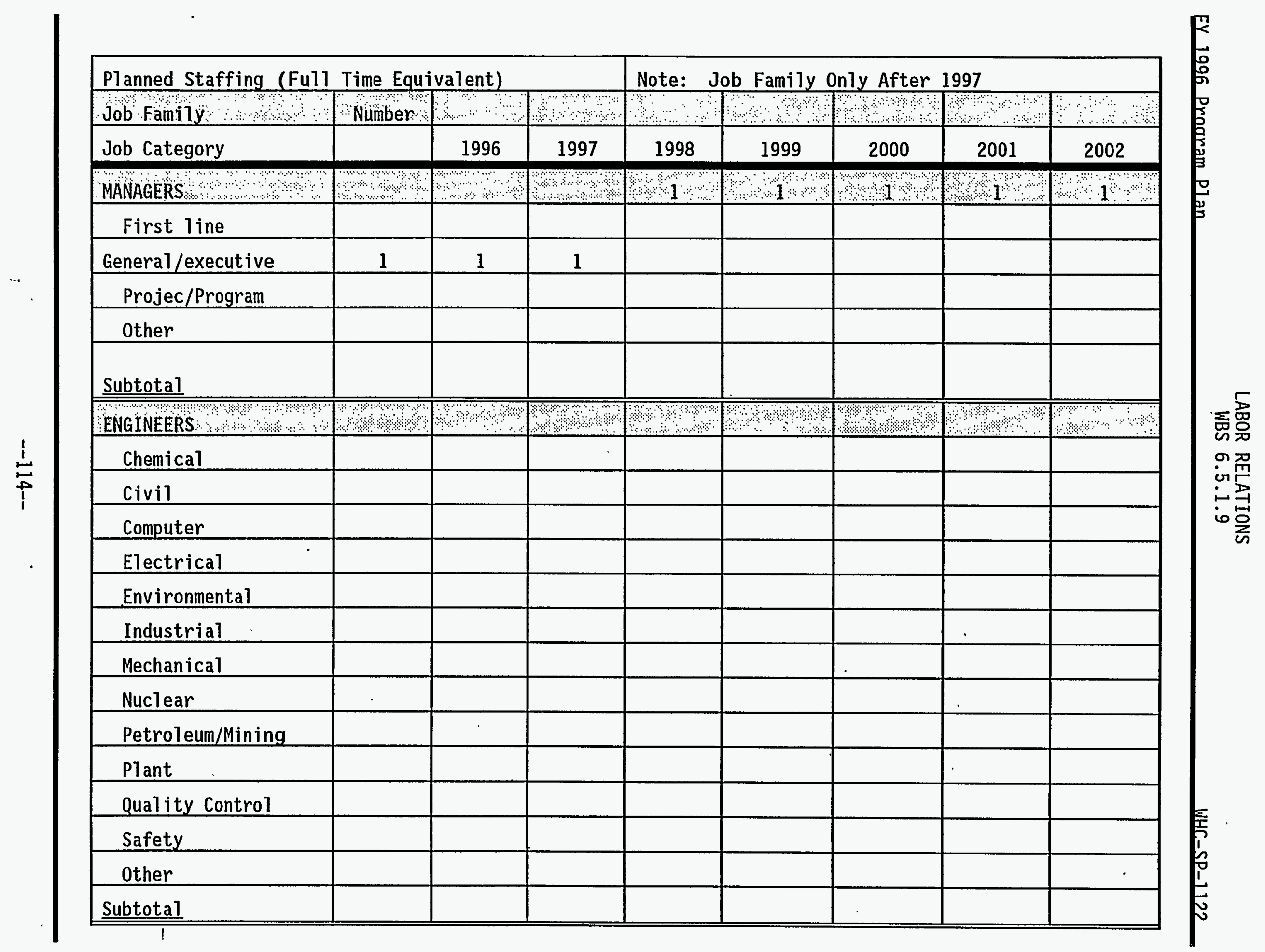




\begin{tabular}{|c|c|c|c|c|c|c|c|c|}
\hline \multicolumn{4}{|c|}{ Planned Staffing (Full Time Equivalent) } & \multicolumn{5}{|c|}{ Note: Job Family Only After 1997} \\
\hline Job Family & Numbers & 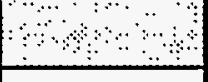 & अ. & sis & अ & «॥ & ४० & 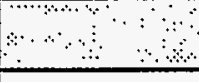 \\
\hline Job Category & & 1996 & 1997 & 1998 & 1999 & 2000 & 2001 & 2002 \\
\hline \multirow{2}{*}{\multicolumn{9}{|c|}{ 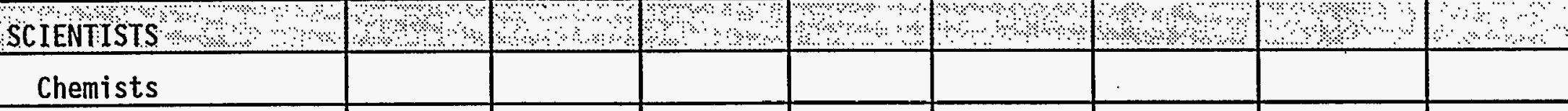 }} \\
\hline & & & & & & & & \\
\hline \multicolumn{9}{|l|}{ Environmental } \\
\hline \multicolumn{9}{|l|}{ Geologists } \\
\hline \multicolumn{9}{|l|}{ Life } \\
\hline \multicolumn{9}{|l|}{ Material } \\
\hline \multicolumn{9}{|l|}{ Mathematicians } \\
\hline \multicolumn{9}{|l|}{ Physicists } \\
\hline \multicolumn{9}{|l|}{ Social } \\
\hline \multicolumn{9}{|l|}{ Other } \\
\hline \multicolumn{9}{|l|}{ Subtotal } \\
\hline $\begin{array}{l}\text { ADMINISTRATIVE } 70 T H E R \\
\text { PROFESSIONALS }\end{array}$ & \%ैं क्ष & अेक & अ०० & 3 & 3 & ४ै। & 3 & $\begin{array}{r}3 \\
3 \\
\end{array}$ \\
\hline \multicolumn{9}{|l|}{ Accountant/auditor } \\
\hline \multicolumn{9}{|l|}{ Architect } \\
\hline \multicolumn{9}{|l|}{ Buyers/Procurement } \\
\hline \multicolumn{9}{|l|}{ Communications } \\
\hline \multicolumn{9}{|l|}{$\begin{array}{l}\text { Compliance } \\
\text { Inspectors }\end{array}$} \\
\hline \multicolumn{9}{|l|}{$\begin{array}{l}\text { Computer Sys } \\
\text { Analyst }\end{array}$} \\
\hline $\begin{array}{l}\text { Cost Est/pianner } \\
\text { /sch }\end{array}$ & & & & & & & & \\
\hline
\end{tabular}




\begin{tabular}{|c|c|c|c|c|c|c|c|c|}
\hline \multicolumn{4}{|c|}{ Planned Staffing (Full Time Equivalent) } & \multicolumn{5}{|c|}{ Note: Job Family Only After 1997} \\
\hline Job Family & Number: & & $\therefore$ & $\because \cdots$ & $8,2 \%$ & अ० & вे & $\cdots$ \\
\hline Job Category & & 1996 & 1997 & 1998 & 1999 & 2000 & 2001 & 2002 \\
\hline \multicolumn{9}{|l|}{ Health Physics } \\
\hline \multicolumn{9}{|l|}{ Industrial Hygiene } \\
\hline \multicolumn{9}{|l|}{ Lawyers } \\
\hline $\begin{array}{l}\text { Personnel/1 abor } \\
\text { Relations }\end{array}$ & 3 & 3 & 3 & & . & & & \\
\hline \multicolumn{9}{|l|}{ Physicians } \\
\hline \multicolumn{9}{|l|}{$\begin{array}{l}\text { Physician } \\
\text { Assistant/Nurse }\end{array}$} \\
\hline \multicolumn{9}{|l|}{$\begin{array}{l}\text { Safeguard \& } \\
\text { Security }\end{array}$} \\
\hline \multicolumn{9}{|l|}{$\begin{array}{l}\text { Tech Writers \& } \\
\text { Editors }\end{array}$} \\
\hline \multicolumn{9}{|l|}{ Trainers } \\
\hline \multicolumn{9}{|l|}{ other } \\
\hline \multicolumn{9}{|l|}{ Subtotal } \\
\hline $\begin{array}{l}\text { GENERAL } \\
\text { ADMIN/SECRETARY/CLERK }\end{array}$ & म & & 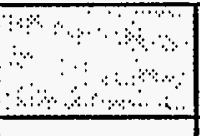 & 3 & $\begin{array}{r}3 \\
3\end{array}$ & 3 & 3 & 3 \\
\hline \multicolumn{9}{|l|}{ Admin Assistant } \\
\hline Office Clerk (Gen) & 2 & 2 & 2 & & & & & \\
\hline \multicolumn{9}{|l|}{$\begin{array}{l}\text { Office Clerk } \\
\text { (Special) }\end{array}$} \\
\hline Secretaries & 1 & 1 & 1 & & & & & \\
\hline \multicolumn{9}{|l|}{$\begin{array}{l}\text { Typist/Word } \\
\text { Processor }\end{array}$} \\
\hline other & & & & & & & & \\
\hline
\end{tabular}




\begin{tabular}{|c|c|c|c|c|c|c|c|c|}
\hline \multicolumn{4}{|c|}{ Planned Staffing (Full Time Equivalent) } & \multicolumn{5}{|c|}{ Note: Job Family Only After 1997} \\
\hline Job Fam 11 y & Number & ४० & ४३\%: & 4 & ४।.? & ४४ڤ & ४। & +s \\
\hline Job Category & & 1996 & 1997 & 1998 & 1999 & 2000 & 2001 & 2002 \\
\hline \multicolumn{9}{|c|}{ Fon; } \\
\hline IECHNICIANS 2 & ४ै। & अ० & अ०\%? & में। & ৷৷ঃ४ & अै। & अे। & \&.s \\
\hline \multicolumn{9}{|l|}{$\begin{array}{l}\text { Computer } \\
\text { Operator/Coder }\end{array}$} \\
\hline \multicolumn{9}{|l|}{ Drafters } \\
\hline \multicolumn{9}{|l|}{ Engineers/Technicians } \\
\hline \multicolumn{9}{|l|}{$\begin{array}{l}\text { Environmental } \\
\text { Science } \\
\text { Technicians } \\
\end{array}$} \\
\hline \multicolumn{9}{|l|}{$\begin{array}{l}\text { Health Physics } \\
\text { Technicians }\end{array}$} \\
\hline \multicolumn{9}{|l|}{$\begin{array}{l}\text { Industrial } \\
\text { Saf/health } \\
\text { Technicians }\end{array}$} \\
\hline \multicolumn{9}{|l|}{$\begin{array}{l}\text { Instru/Control } \\
\text { Technicians }\end{array}$} \\
\hline \multicolumn{9}{|l|}{ Lab. Technicians } \\
\hline \multicolumn{9}{|l|}{ Media Technicians } \\
\hline \multicolumn{9}{|l|}{$\begin{array}{l}\text { Survey/Map } \\
\text { Technicians }\end{array}$} \\
\hline \multicolumn{9}{|l|}{ Other } \\
\hline \multicolumn{9}{|l|}{ Subtotal } \\
\hline CRAFTS $\%$ ' & अ४। & अे। & १०\% & अ & ४ও ২ & ०० & 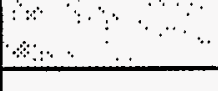 & \\
\hline Carpenters & & & & & & & & \\
\hline
\end{tabular}




\begin{tabular}{|c|c|c|c|c|c|c|c|c|}
\hline \multicolumn{4}{|c|}{ Planned Staffing (Full Time Equivalent) } & \multicolumn{5}{|c|}{ Note: Job Family Only After 1997} \\
\hline Job Family & Number: & a & अं। & 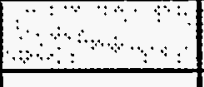 & अे। & ४ै। & «० & अल \\
\hline Job Category & & 1996 & 1997 & 1998 & 1999 & 2000 & 2001 & 2002 \\
\hline \multicolumn{9}{|l|}{ Electricians } \\
\hline \multicolumn{9}{|l|}{ HVAC } \\
\hline \multicolumn{9}{|l|}{ Machinists } \\
\hline \multicolumn{9}{|l|}{ Masons } \\
\hline \multicolumn{9}{|l|}{ Millwrights } \\
\hline \multicolumn{9}{|l|}{ Painters } \\
\hline \multicolumn{9}{|l|}{ Plmbrs/Pipefitters } \\
\hline \multicolumn{9}{|l|}{$\begin{array}{l}\text { Struct/Metal } \\
\text { Workers }\end{array}$} \\
\hline \multicolumn{9}{|l|}{$\begin{array}{l}\text { Vehicl/Mobile } \\
\text { Equip. } \\
\text { Mechanics } \\
\end{array}$} \\
\hline \multicolumn{9}{|l|}{ Welders } \\
\hline \multicolumn{9}{|l|}{ Other } \\
\hline \multicolumn{9}{|l|}{ Subtotal } \\
\hline OPERATORS & \%० & 8 & अ०० & थल & क\% & נe & $8 \cdots$ & $\therefore$ \\
\hline \multicolumn{9}{|l|}{ Lt. Vehicle Drivers } \\
\hline \multicolumn{9}{|l|}{$\begin{array}{l}\text { Material Moving } \\
\text { Equip. }\end{array}$} \\
\hline \multicolumn{9}{|l|}{ Nuclear Plant } \\
\hline \multicolumn{9}{|l|}{$\begin{array}{l}\text { Utilities Waste } \\
\text { Proces }\end{array}$} \\
\hline \multicolumn{9}{|l|}{ other } \\
\hline Subtotal & & & & & & & & \\
\hline
\end{tabular}




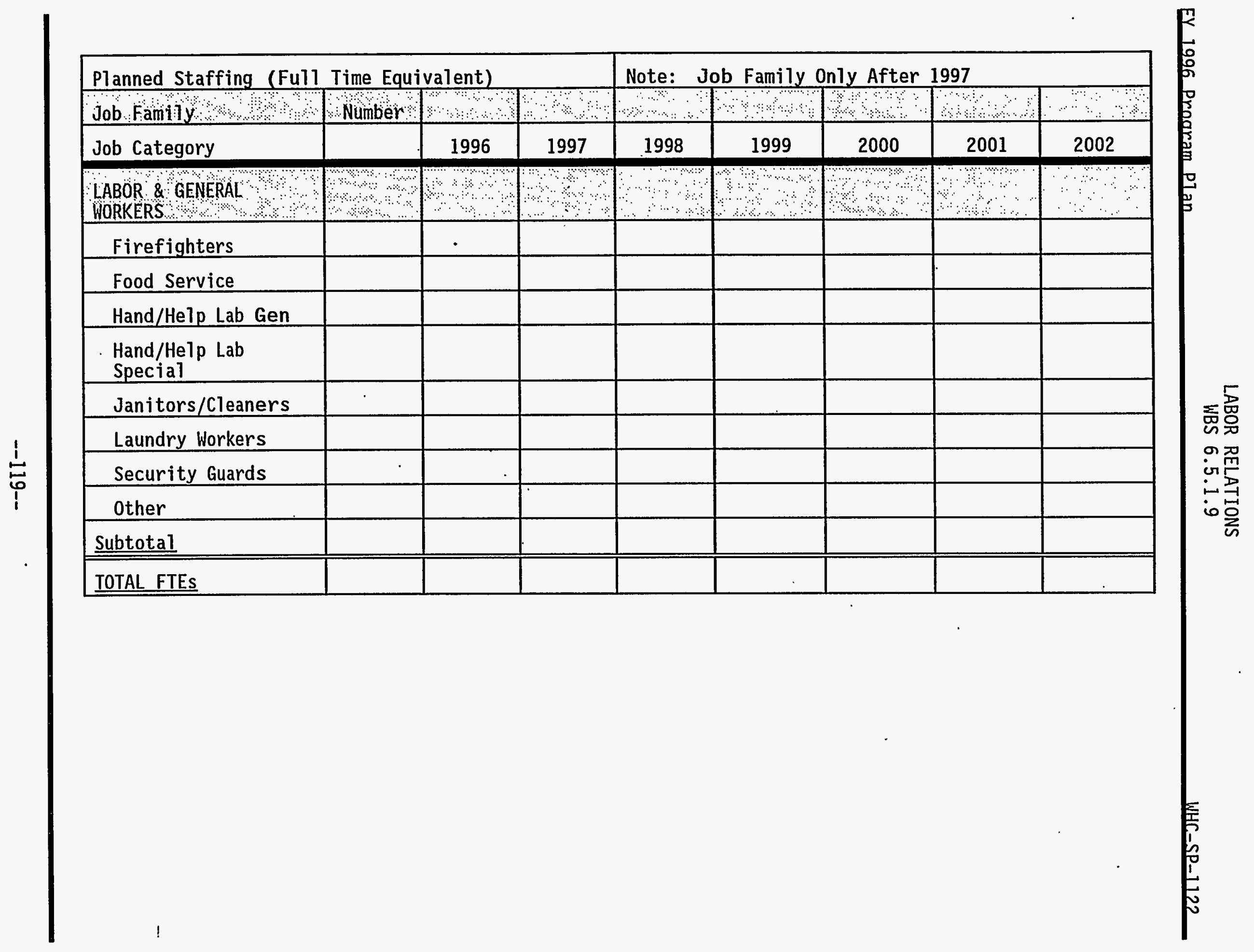




\subsection{SCHEDULE BASELINE}

4.1 Product Deliverables/Milestone List . 


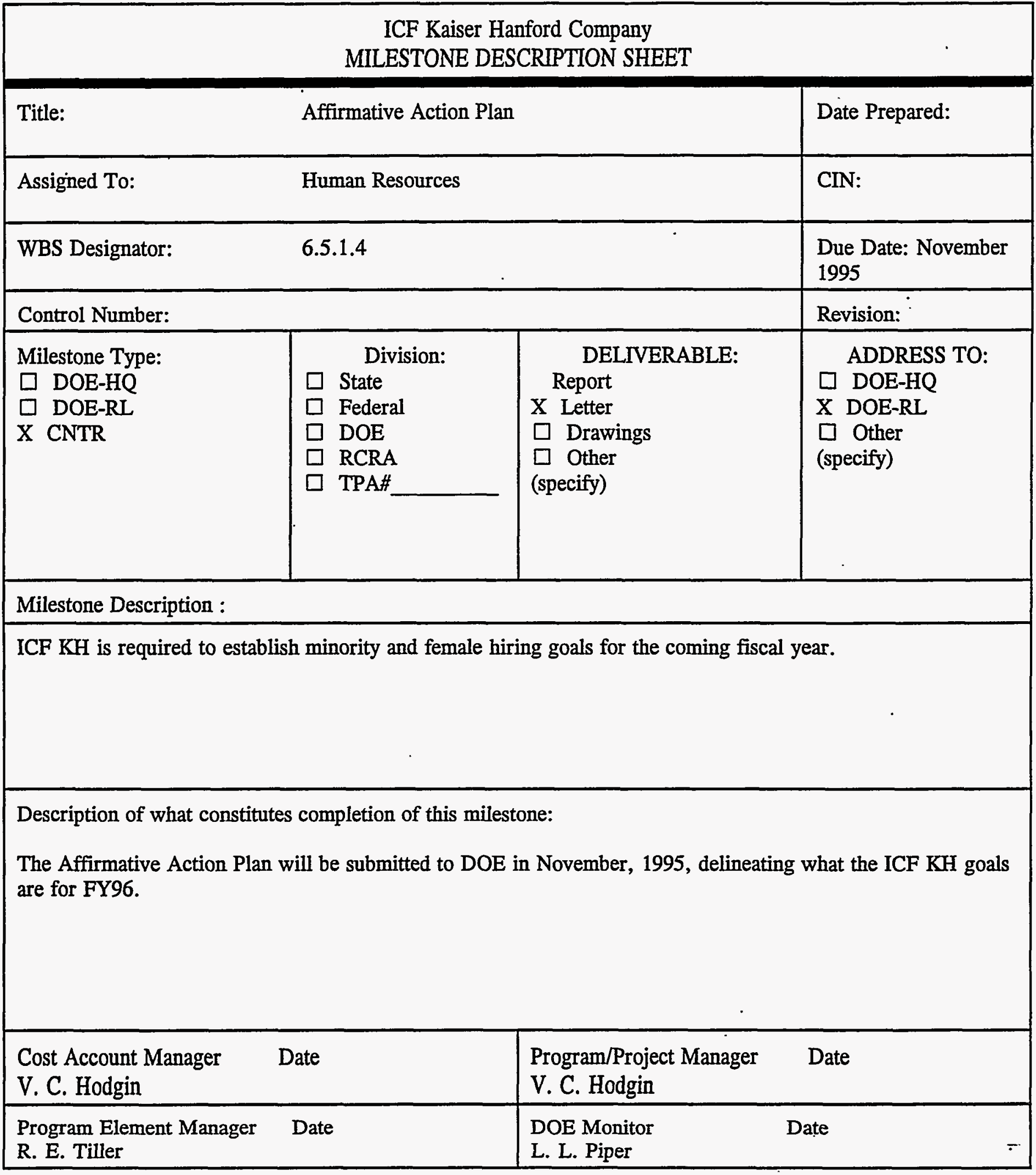




\begin{tabular}{|c|c|c|c|}
\hline \multicolumn{4}{|c|}{$\begin{array}{l}\text { ICF Kaiser Hanford Company } \\
\text { MILESTONE DESCRIPTION SHEET }\end{array}$} \\
\hline Title: & \multicolumn{2}{|c|}{ Performance based incentives (PBIs) } & $\begin{array}{l}\text { Date Prepared: } \\
\text { Aug } 15,1995\end{array}$ \\
\hline Assigned To: & \multicolumn{2}{|c|}{ Organizational Development } & CIN: \\
\hline WBS Designator: & \multicolumn{2}{|l|}{6.5 .1 .5} & Due Date: $9 / 15 / 96$ \\
\hline \multicolumn{3}{|l|}{ Control Number: } & Revision: \\
\hline $\begin{array}{l}\text { Milestone Type: } \\
\square \text { DOE-HQ } \\
\square \text { DOE-RL } \\
\text { X CNTR }\end{array}$ & \begin{tabular}{ll} 
& \multicolumn{1}{c}{ Division: } \\
$\square$ & State \\
$\square$ & Federal \\
$\square$ & DOE \\
$\square$ & RCRA \\
$\square$ & TPA\# \\
\end{tabular} & $\begin{array}{l}\text { DELIVERABLE: } \\
\text { X Report } \\
\square \text { Letter } \\
\square \text { Drawings } \\
\square \text { Other } \\
\text { (specify) }\end{array}$ & $\begin{array}{l}\text { ADDRESS TO: } \\
\square \text { DOE-HQ } \\
\square \text { DOE-RL } \\
\text { X Other } \\
\text { (specify) } \\
\text { ICF KH Corporate }\end{array}$ \\
\hline \multicolumn{4}{|l|}{ Milestone Description : } \\
\hline \multicolumn{4}{|c|}{$\begin{array}{l}\text { Performance based incentives delineating the performance objectives that are incentivized for ICF KH are due } \\
\text { every year prior to the start of the fiscal year. }\end{array}$} \\
\hline \multicolumn{4}{|c|}{$\begin{array}{l}\text { Description of what constitutes completion of this milestone: } \\
\text { Delivery of the PBI's to WHC. }\end{array}$} \\
\hline $\begin{array}{l}\text { Cost Account Manager } \\
\text { W. A. Kitchen }\end{array}$ & Date & $\begin{array}{l}\text { Program/Project Manager } \\
\text { S. W. Bork }\end{array}$ & Date \\
\hline $\begin{array}{l}\text { Program Element Manager } \\
\text { N/A }\end{array}$ & Date & $\begin{array}{l}\text { DOE Monitor } \\
\text { L. L. Piper }\end{array}$ & ate \\
\hline
\end{tabular}




\begin{tabular}{|c|c|c|c|}
\hline \multicolumn{4}{|c|}{$\begin{array}{l}\text { ICF Kaiser Hanford Company } \\
\text { MILESTONE DESCRIPTION SHEET }\end{array}$} \\
\hline Title: & \multicolumn{2}{|c|}{ Performance Evaluation Plan (PEP) } & $\begin{array}{l}\text { Date Prepared: } \\
\text { Aug 08, } 1995\end{array}$ \\
\hline Assigned To: & \multicolumn{2}{|c|}{ Organizational Development } & CIN: \\
\hline WBS Designator: & \multicolumn{2}{|l|}{6.5 .1 .5} & Due Date: $9 / 15 / 96$ \\
\hline \multicolumn{3}{|l|}{ Control Number: } & Revision: \\
\hline $\begin{array}{l}\text { Milestone Type: } \\
\square \text { DOE-HQ } \\
\square \text { DOE-RL } \\
\text { X CNTR }\end{array}$ & \begin{tabular}{ll}
\multicolumn{1}{c}{ Division: } \\
$\square$ State \\
$\square$ Federal \\
$\square$ DOE \\
$\square$ RCRA \\
$\square$ TPA\#
\end{tabular} & $\begin{array}{l}\text { DELIVERABLE: } \\
\square \text { Report } \\
\square \text { Letter } \\
\square \text { Drawings } \\
\text { X Other } \quad \text { (specify) } \\
\text { PEP }\end{array}$ & $\begin{array}{l}\text { ADDRESS TO: } \\
\square \text { DOE-HQ } \\
\square \text { DOE-RL } \\
\text { X Other } \\
\text { (specify) } \\
\text { WHC }\end{array}$ \\
\hline \multicolumn{4}{|l|}{ Milestone Description : } \\
\hline \multicolumn{4}{|c|}{$\begin{array}{l}\text { Performance Evaluation Plans delineating the performance goals, objectives, and criteria for ICF } \mathrm{KH} \text { are due } \\
\text { every six (6) months prior to the start of the award fee period. }\end{array}$} \\
\hline \multicolumn{4}{|c|}{$\begin{array}{l}\text { Description of what constitutes completion of this milestone: } \\
\text { Delivery of the ICF KH PEP to WHC. }\end{array}$} \\
\hline $\begin{array}{l}\text { Cost Account Manager } \\
\text { W. A. Kitchen }\end{array}$ & Date & $\begin{array}{l}\text { Program/Project Manager } \\
\text { S. W. Bork }\end{array}$ & Date \\
\hline $\begin{array}{l}\text { Program Element Manage } \\
\text { N/A }\end{array}$ & Date & $\begin{array}{l}\text { DOE Monitor } \\
\text { L. L. Piper }\end{array}$ & ate \\
\hline
\end{tabular}




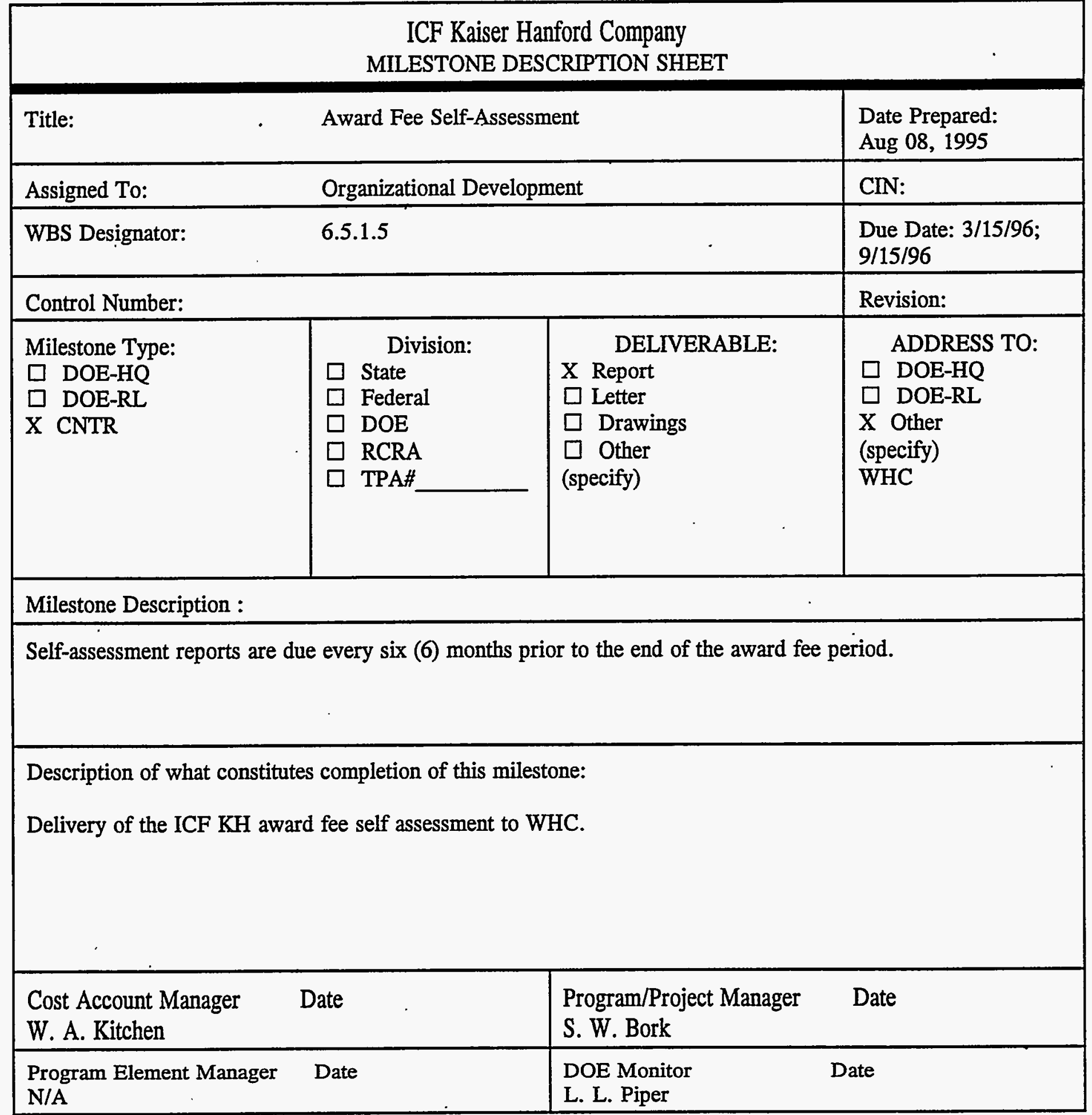


5.0 COST BASELINE SUMMARY

5.1 Cost Baseline

5.2 Staffing Profile 

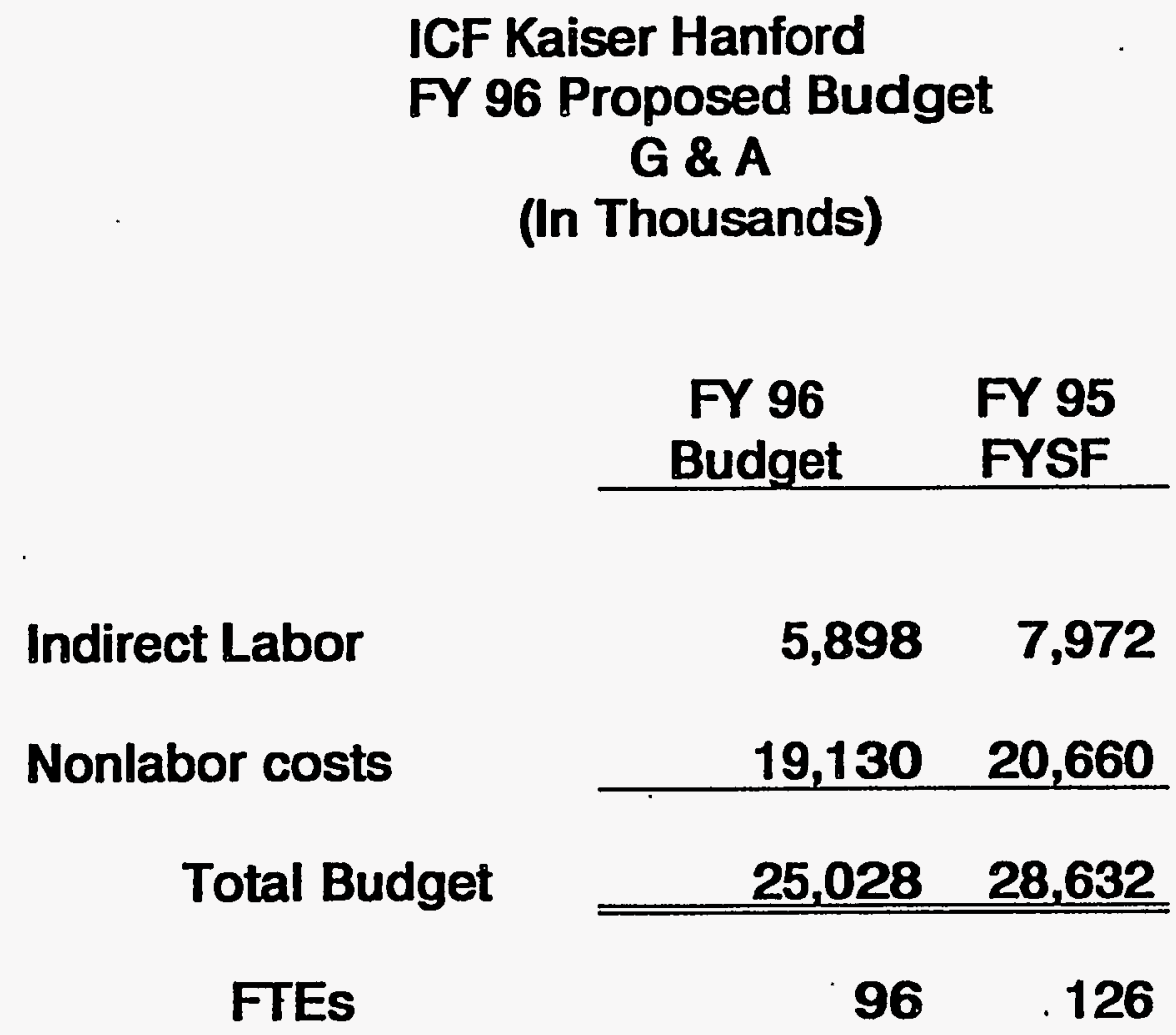
'FY 1000 BLOCET PREPARUTION ORGANIZATION: rofkH GM

CE DESCAPTION

ONOW Misc UABOR

$\infty$ NON EXEMPT-DOULAR

01 EXEMPT - DOLIARS - RM

02 NON EEEMPTO ON SUPport

03

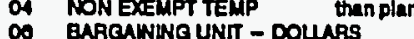

D - HEADS

BARo UNT OT

TOTAL UBOA DOLLARS TOTAL REQ HEADS

10 MATERLLSIEOUIPMENT

13 TOOLS E BAFETY

14 OFFICE SUPPLEES

15 BHOP AND LAB

10 ADP EOUP - H/W

TOTAL MTERU

$2 F$ ADP EQUP LEASE

20 AOP EOUP MINT

2H ADP BN - LSE LMN

2L ON BITE DEV TANG

2N EDUCATONULREIM

2R RELOCATION

2T TPUVELLLMNG

2X PAOF R TECH 8OC TRV

$2 Y$ LOCAL MTOS L CONF

21 P.O. CONTPUCTS

20 MISC MEMEERSHPS

20 MISC MEMTH ERTCS

TOTAL PURCH BAVCS

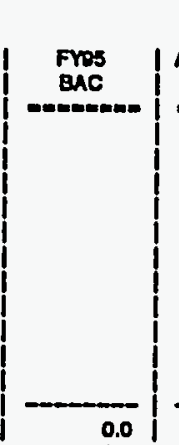

OROS

AOAMSON OLSEN

IMKOAB

KTTCHEN ARSANUN

IMOAC TMROAE

Cor25/2s =-mon-

$\begin{array}{rr}23.0 & 23.0 \\ 1.0 & 1.0\end{array}$

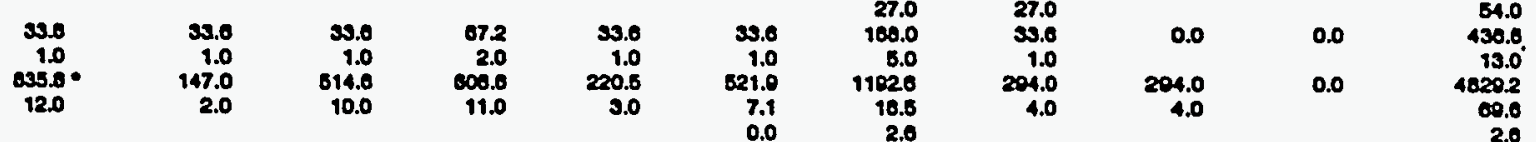

$30.0 \quad 672$

MOVE HODGIN ANDERSON CARTER TILER ASSESSMENTS

coras

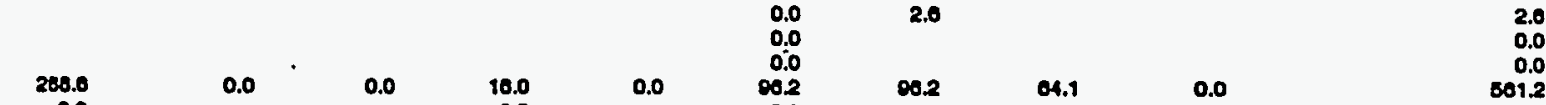

8.1

180.0
3.0

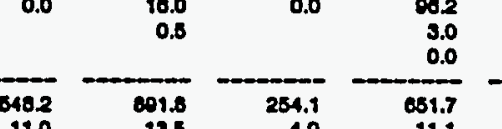

00.2
3.0
0.2
1405.0

\begin{tabular}{r}
2.0 \\
\hline 410.0 \\
7.0 \\
0.0
\end{tabular}

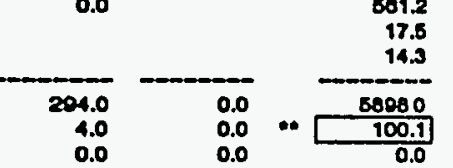

悬
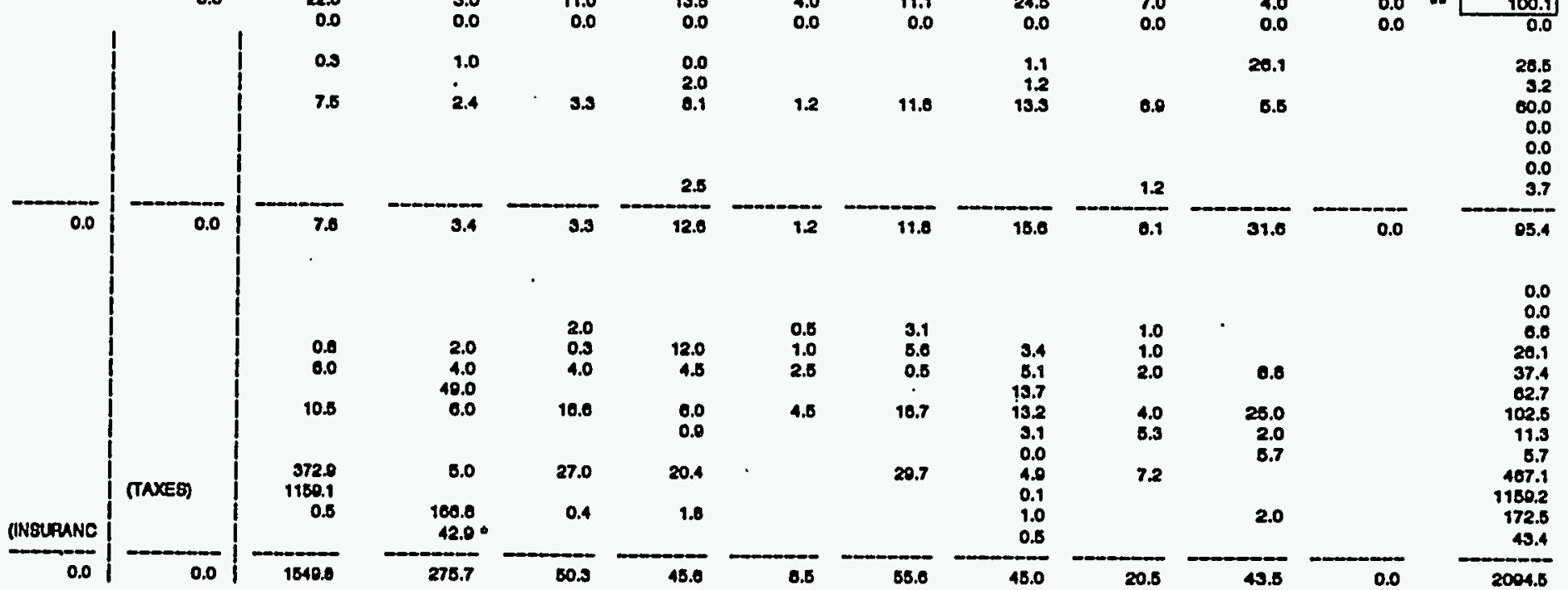

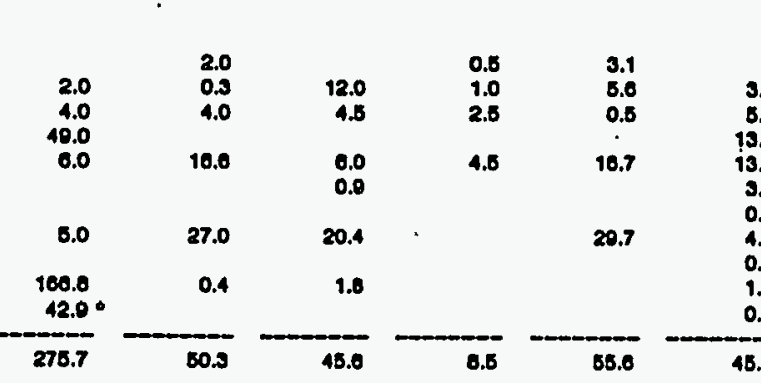

$\begin{array}{ll} & \\ 3.4 & 1.0 \\ 5.1 & 1.0 \\ 3.7 & 2.0 \\ 3.2 & 4.0 \\ 3.1 & 5.3 \\ 0.0 & \\ 4.0 & 7 \\ 0.1 & \\ 1.0 & \end{array}$

$\begin{array}{lr}1.0 & \\ 1.0 & \\ 2.0 & \\ 4.0 & 25.0 \\ 8.3 & 2.0 \\ 7.2 & 6.7 \\ & \\ & 2.0\end{array}$

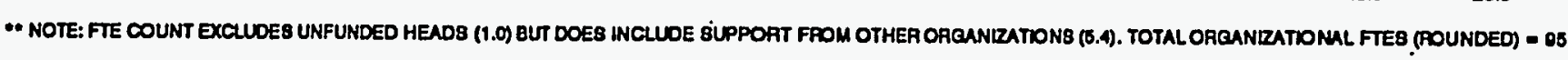

$-19.5-\frac{0.0}{0.0}$

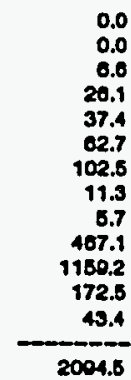


FY 1000 BLOGET PREPARATON OACANIZATION: KCFIOH CM

EE DEscapton

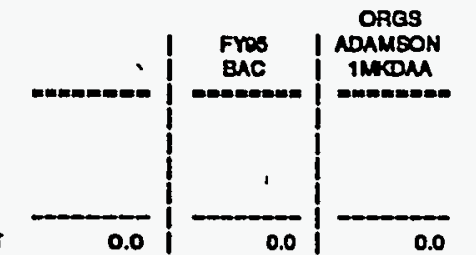

30 PNLOTHER

3M HEHF

TOTAL OTHER CONT NET ORIGINATEO COST

1C MATL PAOC RATE RMPFO

D gTANDARDS LAB

IJ BUSOPERATONSALOC

CQUNEAND RHBGHE

LAUNDAY

HUMMER TRUININo

LOCKSMITHS

$4 P$ EQUIPMENT MOVES

IV ROADSIGROUNDS

4W OT LUNCHES

41/40 MANDATORY TRANINO TOTAL BITE BANCS

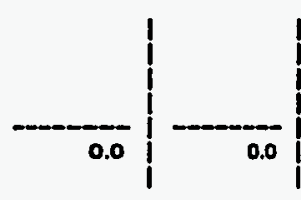

BD DOSIMETaY
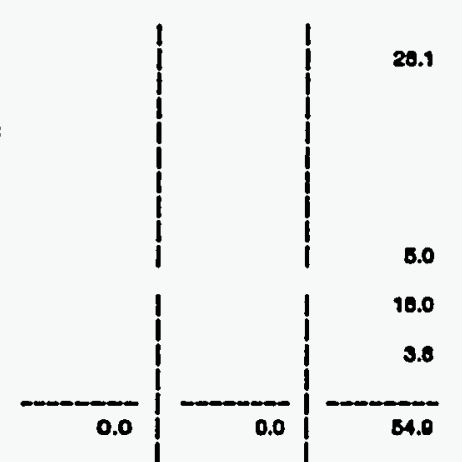

20.1

GF2 MANMGEMENT PAORATON AZ WABTE HANDLNO TOTAL. INTERMLL WHC

a CFCO - BM

OJ EUCASSESSMEN

MDPE MAINT

NETWOAKACCESS

OT PLANT TELEPHONE

MISC RM COST8

TOTAL RM aFNCS

$7 C \mathrm{ORO} . \mathrm{OH}$

TOTALORO.OH

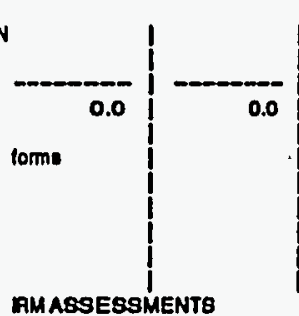

OLSEN

KTTCHEN ARSUANUN LOVE

IMKOAB TMOAC TMOAE IMNOAF

HODGIN ANDERSON CARTER THLER ASSESSMENTS

IMKOAG TMOAH TMEOAL IMKOAS IMKOAJ

corasos

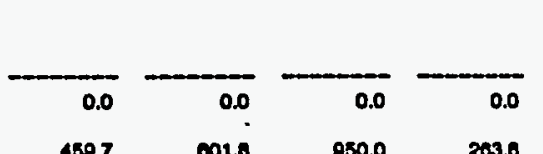

$\begin{array}{r}0.0 \\ -\quad 0.0 \\ \hline\end{array}$

0.2

48

4.0

0.2

0.2

0.2

23.

3.1

2.9

4.7

0.0
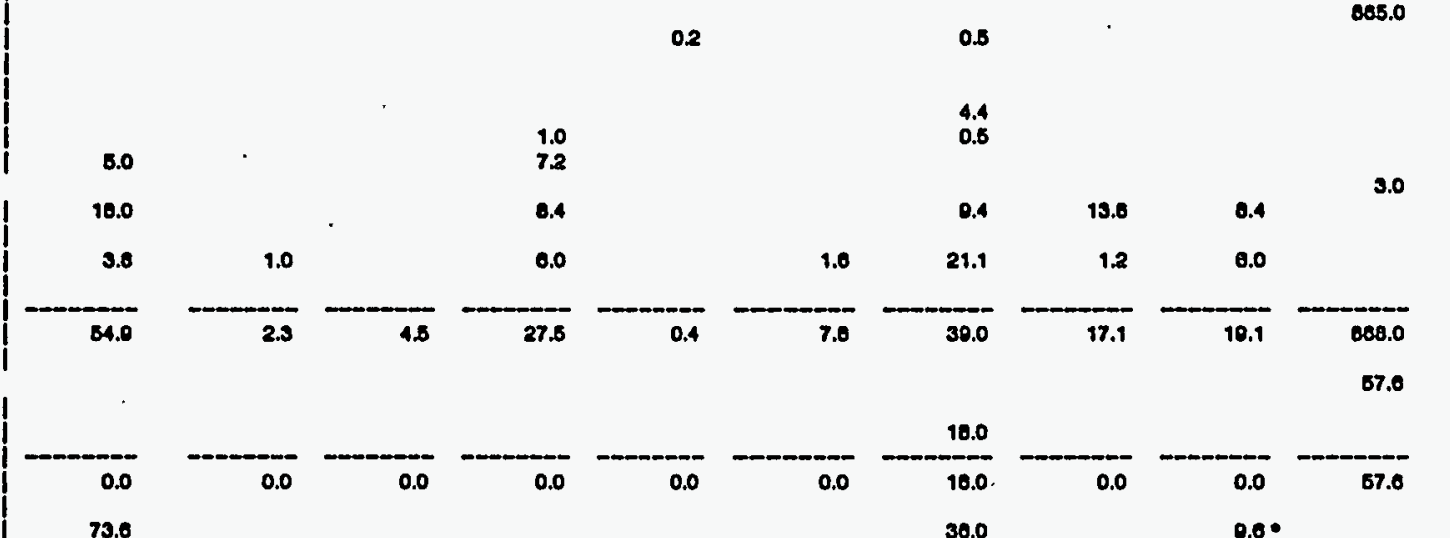

0.0

$0.0 *$$$
\text { - }
$$

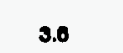

30
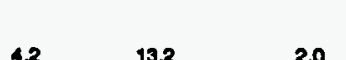

3.6

3.0

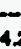

$42-13.2$
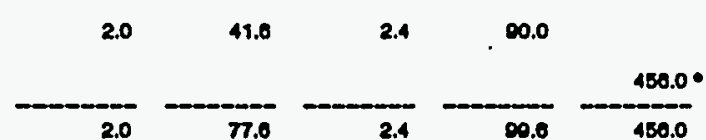
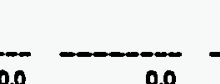

\begin{tabular}{rrr}
42.8 & $18.0 \quad 15000.0$ \\
\hline-42.0 & 10.0 & 18000.0
\end{tabular}

465.0 $\quad 000.5 \quad 061.7 \quad 277.4$

$\begin{array}{r}728.0 \\ -2.0 .0 \\ \hline\end{array}$

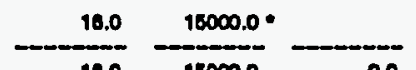

TOTAL

$$
\text { PAREL }
$$

veriance 


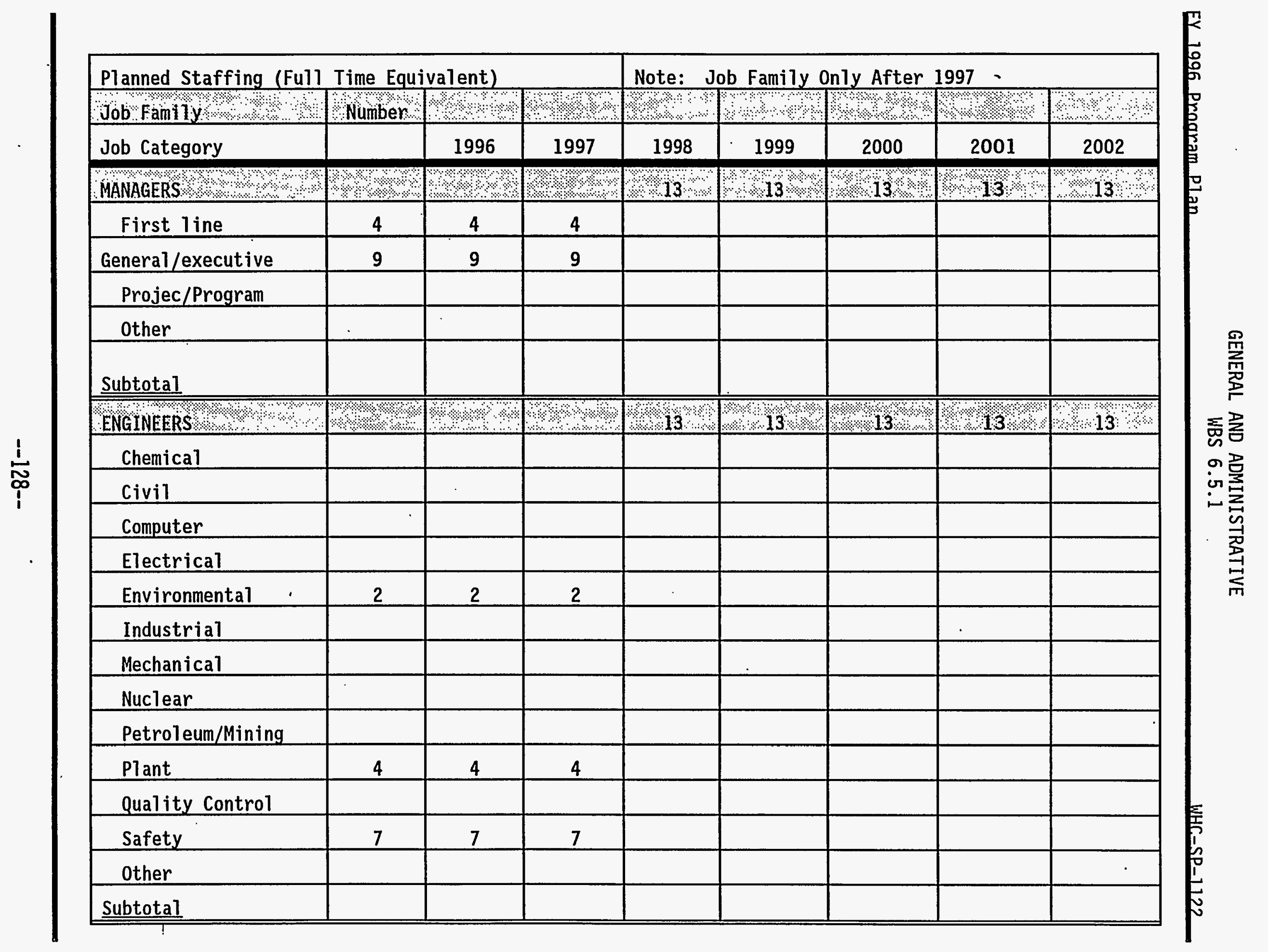




\begin{tabular}{|c|c|c|c|c|c|c|c|c|}
\hline \multicolumn{4}{|c|}{ Planned Staffing (Full Time Equivalent) } & \multicolumn{5}{|c|}{ Note: Job Family Only After 1997} \\
\hline Job Famfly y & Number: & 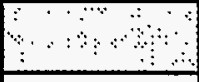 & \% & sis: & अं। & \%ै। & \%०: & s. \\
\hline Job Category & & 1996 & 1997 & 1998 & 1999 & 2000 & 2001 & 2002 \\
\hline SCIENTISTS & ४ै? & 3 & अे & 6 & अ6! & 6, & 8,6 & अ 6. \\
\hline Chemists & & & & & & & & \\
\hline Environmental & 6 & 6 & 6 & & & & & \\
\hline Geologists & & & & & & & & \\
\hline Life & & & & & & & & \\
\hline Material & & & & & & & & \\
\hline Mathematicians & & & & . & & & & \\
\hline Physicists & & & & & & & & \\
\hline Social & & & & & & & & \\
\hline other & & & & & & & & \\
\hline Subtotal & & & & & & & & \\
\hline $\begin{array}{l}\text { ADMINISTRATIVE OTTHER } \\
\text { PROEESSIONALS }\end{array}$ & \%? & \%ै। & \%ै\% & 32 & अै 32 & +32\%? & 32 & 32 \\
\hline Accountant/auditor & 10 & 10 & 10 & & & & & \\
\hline Architect & & & & & & & & \\
\hline Buyers/Procurement & & & & & & & & \\
\hline Communications & 3 & 3 & 3 & & & & & \\
\hline $\begin{array}{l}\text { Compl } 1 \text { iance } \\
\text { Inspectors }\end{array}$ & 2 & 2 & 2 & & & & & \\
\hline $\begin{array}{l}\text { Computer Sys } \\
\text { Analyst }\end{array}$ & & . & & & & & & \\
\hline $\begin{array}{l}\text { Cost Est/planner } \\
\text { /sch }\end{array}$ & & & & & & & & \\
\hline
\end{tabular}




\begin{tabular}{|c|c|c|c|c|c|c|c|c|}
\hline \multicolumn{4}{|c|}{ Planned Staffing (Full Time Equivalent) } & \multicolumn{5}{|c|}{ Note: Job Family Only After 1997} \\
\hline Job Family & Number: & we & ? & एक। & ४४ & ४ै० & ॐैे & थ. \\
\hline Job Category & & 1996 & 1997 & 1998 & 1999 & 2000 & 2001 & 2002 \\
\hline \multicolumn{9}{|l|}{ Health Physics } \\
\hline \multicolumn{9}{|l|}{ Industrial Hygiene } \\
\hline \multicolumn{9}{|l|}{ Lawyers } \\
\hline $\begin{array}{l}\text { Personnel/1abor } \\
\text { Relations }\end{array}$ & 9 & 9 & 9 & & & & & \\
\hline \multicolumn{9}{|l|}{ Physicians } \\
\hline \multicolumn{9}{|l|}{$\begin{array}{l}\text { Physician } \\
\text { Assistant/Nurse }\end{array}$} \\
\hline $\begin{array}{l}\text { Safeguard \& } \\
\text { Security }\end{array}$ & 1 & 1 & 1 & & & & & \\
\hline $\begin{array}{l}\text { Tech Writers \& } \\
\text { Editors }\end{array}$ & 1 & 1 & 1 & & & & & \\
\hline Trainers & .3 & 3 & 3 & & & & & \\
\hline other & 3 & 3 & 3 & & & & & \\
\hline \multicolumn{9}{|l|}{ Subtotal } \\
\hline $\begin{array}{l}\text { GENERAL } \\
\text { ADMIN/SECRETARY/CLERK }\end{array}$ & \%? & \%ै? & $1 \% ?$ & 31.5 & 31.5 & 31.5 & 31,5 & 31,5 \\
\hline Admin Assistant & 3 & 3 & 3 & & & & & \\
\hline Office Clerk (Gen) & 20.5 & 20.5 & 20.5 & & & & & \\
\hline \multicolumn{9}{|l|}{$\begin{array}{l}\text { Office Clerk } \\
\text { (Special) }\end{array}$} \\
\hline Secretaries & 8 & 8 & 8 & & & & & \\
\hline \multicolumn{9}{|l|}{$\begin{array}{l}\text { Typist/Word } \\
\text { Processor }\end{array}$} \\
\hline other & & & & & & & & \\
\hline
\end{tabular}




\begin{tabular}{|c|c|c|c|c|c|c|c|c|}
\hline \multicolumn{4}{|c|}{ Planned Staffing (Full Time Equivalent) } & \multicolumn{5}{|c|}{ Note: Job Family Only After 1997} \\
\hline Job Fam ly & Number & म, & ४४४४ & अ०। & ২॰ ४ै। & $3 .+\%$ & ४ै। ४) & ২২৪ \\
\hline Job Category & & 1996 & 1997 & 1998 & 1999 & 2000 & 2001 & 2002 \\
\hline \multicolumn{9}{|l|}{ Subtotal } \\
\hline IECHNICIANS & 4 & अ००: & ২ঙ.ি & अ.। & अ 1 ?ै। & २ै 1 ? & २ै। 1 अे & में \\
\hline \multicolumn{9}{|l|}{$\begin{array}{l}\text { Computer } \\
\text { Operator/Coder }\end{array}$} \\
\hline \multicolumn{9}{|l|}{ Drafters } \\
\hline Engineers/Technicians & 1 & 1 & 1 & & & & & \\
\hline \multicolumn{9}{|l|}{$\begin{array}{l}\text { Environmental } \\
\text { Science } \\
\text { Technicians }\end{array}$} \\
\hline \multicolumn{9}{|l|}{$\begin{array}{l}\text { Health Physics } \\
\text { Technicians }\end{array}$} \\
\hline \multicolumn{9}{|l|}{$\begin{array}{l}\text { Industrial } \\
\text { Saf/health } \\
\text { Technicians }\end{array}$} \\
\hline \multicolumn{9}{|l|}{$\begin{array}{l}\text { Instru/Control } \\
\text { Technicians }\end{array}$} \\
\hline \multicolumn{9}{|l|}{ Lab. Technicians } \\
\hline \multicolumn{9}{|l|}{ Media Technicians } \\
\hline \multicolumn{9}{|l|}{$\begin{array}{l}\text { Survey/Map } \\
\text { Technicians }\end{array}$} \\
\hline \multicolumn{9}{|l|}{ Other } \\
\hline \multicolumn{9}{|l|}{ Subtotal } \\
\hline GRAFIS & मे०े। & अेै। & ४ै। & \%े। & ४ै। & ২ै। & अ. & s: \\
\hline \multicolumn{9}{|l|}{ Carpenters } \\
\hline Electricians & & & & & & & & \\
\hline
\end{tabular}




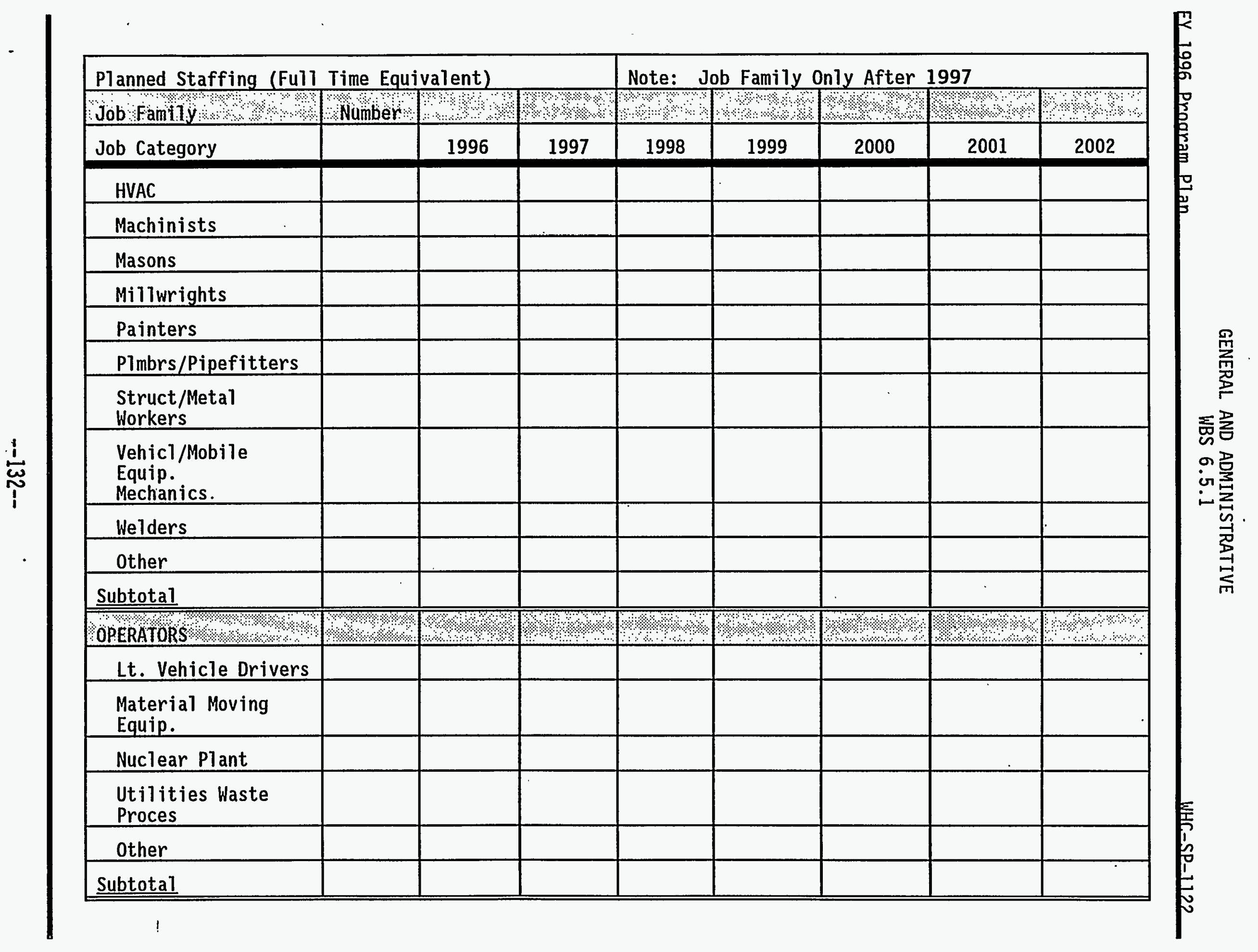




\begin{tabular}{|c|c|c|c|c|c|c|c|c|}
\hline \multicolumn{4}{|c|}{ Planned Staffing (Full Time Equivalent) } & \multicolumn{5}{|c|}{ Note: Job Family Only After 1997} \\
\hline Job famfly & Number: & ४४ & ঔ৬। & $\%:$ & ৪४४ & अथ & अ. & « \\
\hline Job Category & & 1996 & 1997 & .1998 & 1999 & 2000 & 2001 & 2002 \\
\hline BABOR \& GENERAL & अै. & +ै। & ४ै। & अै। & ?ै: & ২ै. & अ & अे \\
\hline Firefighters & & & & & & & & \\
\hline Food Service & & & & & & & & \\
\hline Hand/Help Lab Gen & & & & & & & & \\
\hline $\begin{array}{l}\text { Hand/Help Lab } \\
\text { Special }\end{array}$ & & & & & & & & \\
\hline Janitors/Cleaners & & & & & & & & \\
\hline Laundry Workers & & & & & & & & \\
\hline Security Guards & & & & & & & & \\
\hline Other & & & & & & & & \\
\hline Subtotal & & & & & & & & \\
\hline TOTAL FTES & & & & & & & & \\
\hline
\end{tabular}




\section{INDEX}

\subsection{CONSTRUCTION MANAGEMENT POOL}

2.0 CONTRACT SERVICES OVERVIEW (WBS 6.5.2)

$2.1 \quad$ Mission

2.2 Assumptions

2.3 Constraints

2.4 Goals and Objectives

2.5 Products/Services

2.6 Primary Customers

\subsection{TECHNICAL REQUIREMENT BASELINE}

\subsection{Work Breakdown Structure}

4.0 SCHEDULE BASELINE
4.1 Product Deliverables/Milestone List

5.0. COST BASELINE SUMMARY

5.1 Cost Baseline

5.2 Planned Staffing Profile 


\subsection{CONSTRUCTION MANAGEMENT POOL}

The primary function of the Construction Management Pool is the placement and administration of Fixed Price Construction contracts, A/E Service Agreements, and miscellaneous specialty contracts.

\subsection{CONSTRUCTION SERVICES (6.5.2)}

\subsection{Mission}

Contract Services: The mission of the ICF KH Contract Services Department is to manage all ICF $\mathrm{KH}$ subcontracted work by performing constructability reviews, planned work activities, and in the preparation and administration of the bid package.

Note: To accomplish this mission, the Contract Services personnel have been assigned to facilitate requests and requirements of project/program organizations.

\subsection{Assumptions}

The following are key assumptions used to develop goals and strategic (building blocks): Reduction of cost is paramount to success and competitive posturing. Outsourcing of work will be conducted on a cost/performance advantage basis, funding and staffing levels will be adequate, re-engineering initiatives will be fully supported, award fee and incentive fee criteria will be established, cost savings programs will be supported, work will comply with current regulations and guidance, and the Voluntary Protection Program will continue.

\subsection{Constraints}

Items that may impede achievements of goals and strategies are as follows: privatization and outsourcing of services, funding support to ICF KH must be adequate for the scope required by DOE, contradictory DOE missions, scheduling/cost software programs changed, Prioritization of work due to budget constraints, and continued company reorganizations.

\section{$2.4 \quad$ Goals and Objectives}

The goals and objectives of the Contract Services department are:

- $\quad$ Continue to develop strong customer relationships inside and outside ICF KH, by being more proactive in our approaches to customer needs and requirements.

- Support process improvements initiatives by providing meaningful measurements to ICF KH personnel that stimulate concern and encourage proactive solutions.

- Based upon reality of reduced staff levels, put emphasis on identifying alternatives manner. Review staff mix to align staff skills/strengths.

- Continue to enhance employee skills and abilities through cross-training and appropriate classes.

- Identify alternative methods of performing work activities. 
- $\quad$ Support the Re-engineering efforts.

- Conduct all work in a safe and cost-efficient manner.

- Continue to bring subcontracting practice in line with commercial based contracting.

2.5 Product and Services

The Contract Services Department provides the following: Procurement Services which includes plan acquisition, preparation of bid lists, preparation of bid packages, solicitation and evaluation of bids, analyzation of cost/price, contract award, and administration of procurement changes; Administers sub-contracts to ensure all contractural requirements are met; Negotiate contract modification; Approve contractor progress payment; and close out contracts.

\subsection{Primary Customers}

The primary customers of the Contract Services Department are: ICF Kaiser Hanford, Westinghouse Hanford Company, Pacific Northwest Laboratory, Outside contractors, and the U.S. Department of Energy (RL). 


\subsection{TECHNICAL REQUIREMENT BASELINE}

\subsection{Work Breakdown Structure}




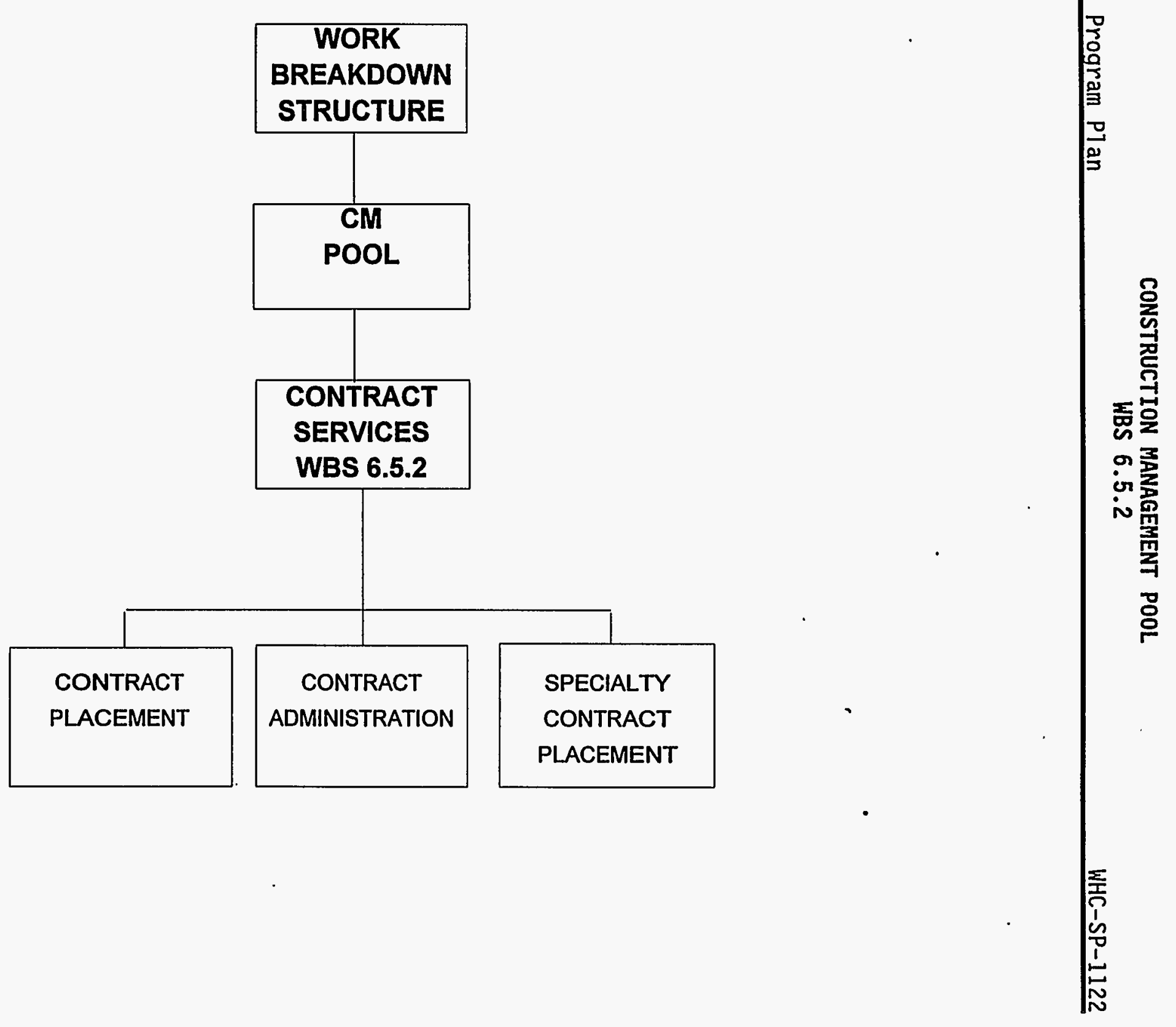


ONSTRUCTION MANAGEMENT POOL (6.5.2)

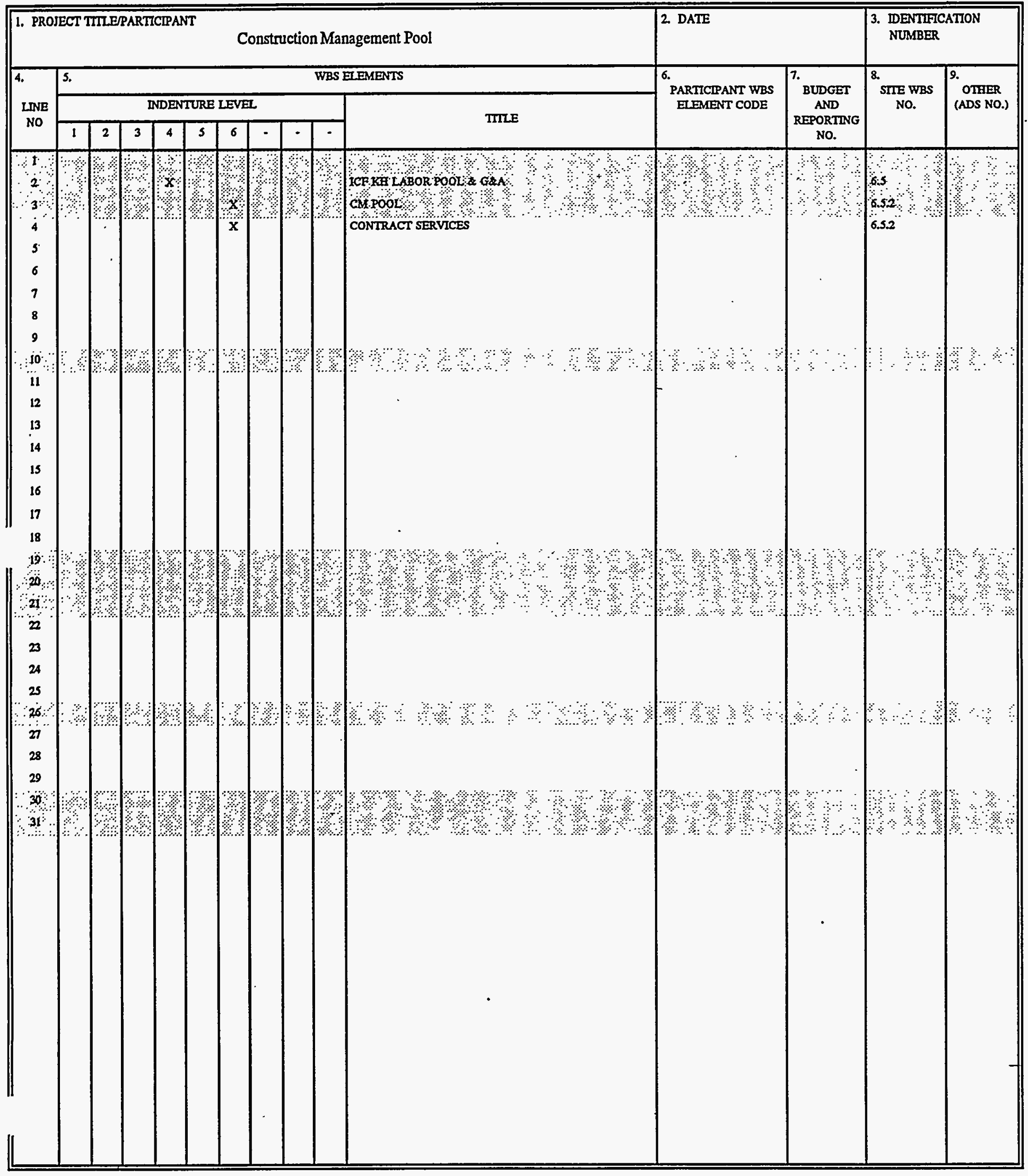




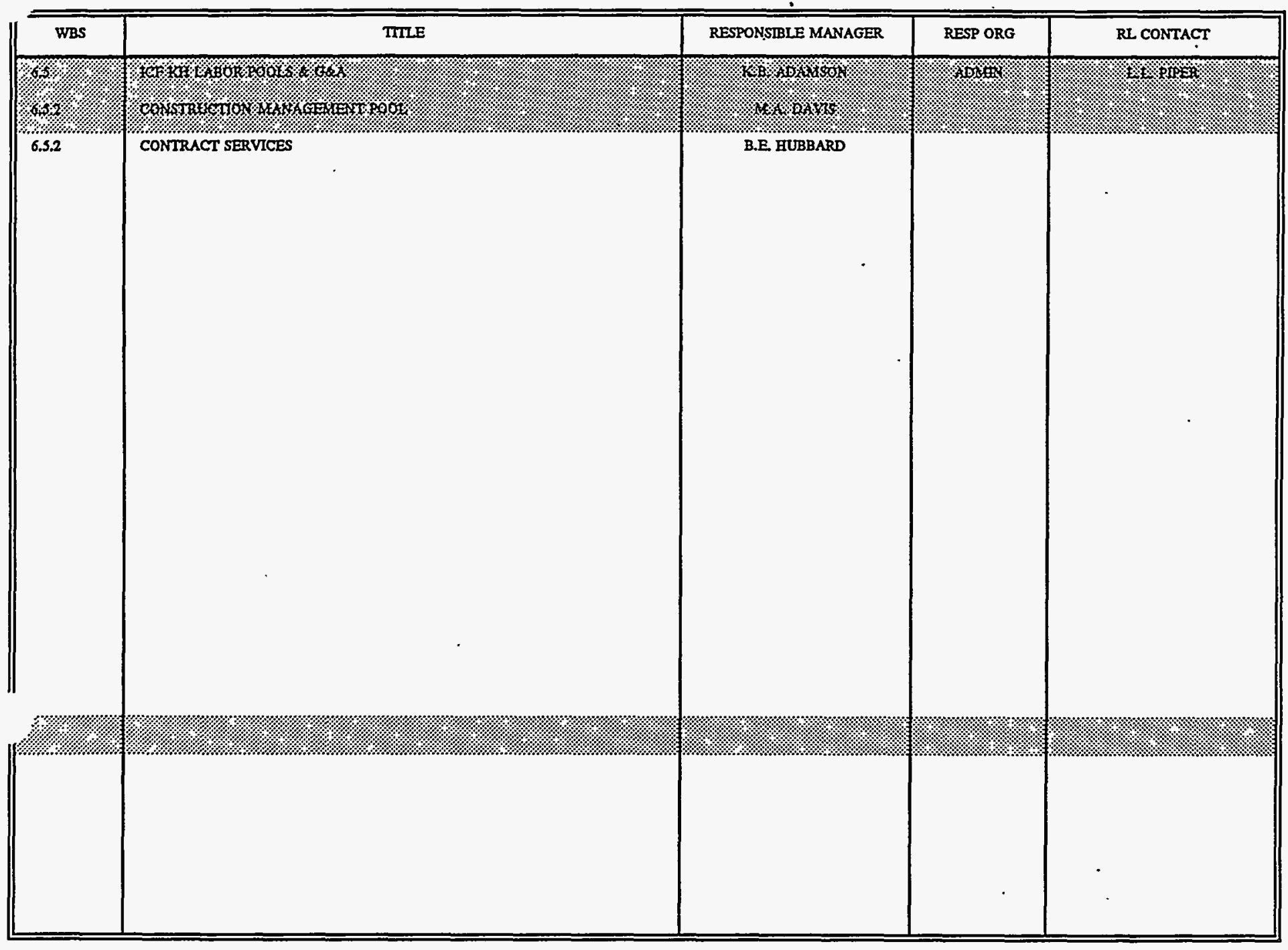


Table 3-1. FY 1996 SSPP CONSTRUCTION MANAGEMENT POOL WBS Dictionary (6.5.2)

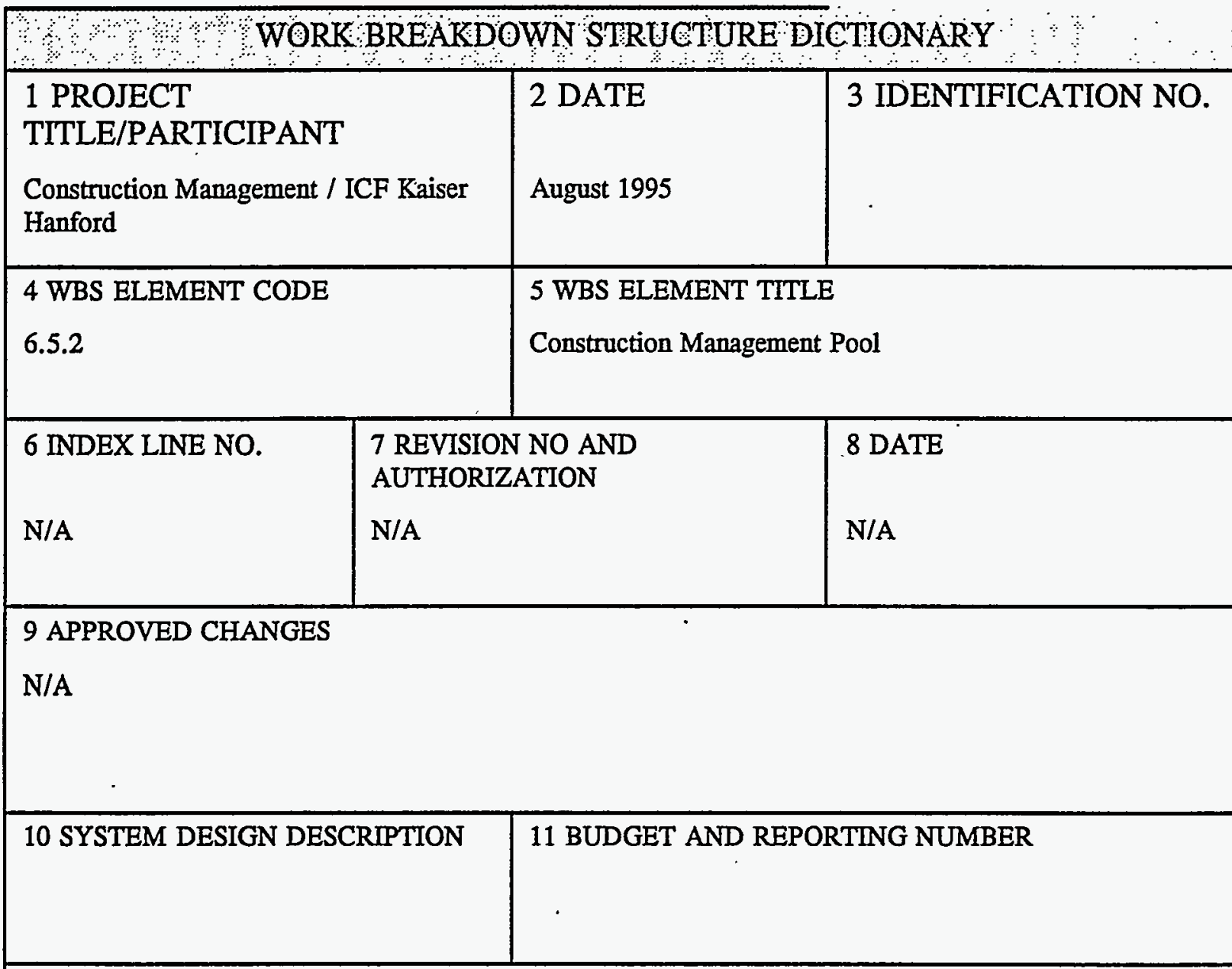

12 ELEMENT TASK DESCRIPTION

The objective of this effort is to manage and execute the scope associated with the Construction Management Pool. It encompasses the Contract Service Department which manages all of the ICF KH Sub-Contracted work.

\section{A. COST CONTENT}

Cost content includes the following cost types: Labor, material, equipment, engineering services, and sub contractors.

\section{B. TECHNICAL CONTENT/WORK STATEMENT}

Complete agreed upon scope identified in the approved SSPP in accordance with applicable regulations, requirements, and DOE Orders. 


\section{CONSTRUCTION MANAGEMENT POOL}

WBS 6.5 .2

FY 1996 Program PTan

WHC-SP-1122

$\because 0$ SCHEDULE BASELINE

4.1 Product Deliverables/Milestone List 


\begin{tabular}{|c|c|c|c|}
\hline \multicolumn{4}{|c|}{$\begin{array}{l}\text { ICF Kaiser Hanford Company } \\
\text { MILESTONE DESCRIPTION SHEET }\end{array}$} \\
\hline Title: & \multicolumn{2}{|c|}{ Socioeconomic Goals Report } & $\begin{array}{l}\text { Date Prepared: } \\
\text { Aug, } 1995\end{array}$ \\
\hline Assigned To: & \multicolumn{2}{|c|}{ Contract Services } & CIN: \\
\hline WBS Designator: & \multicolumn{2}{|l|}{6.5 .2} & $\begin{array}{l}\text { Due Date: } 4 / 25 \text {, } \\
10 / 25\end{array}$ \\
\hline \multicolumn{3}{|l|}{ Control Number: } & Revision: \\
\hline $\begin{array}{l}\text { Mitestone Type: } \\
\square \text { DOE-HQ } \\
\text { X DOE-RL } \\
\square \text { CNTR }\end{array}$ & $\begin{array}{ll} & \text { Division: } \\
\square & \text { State } \\
\square & \text { Federal } \\
\square & \text { DOE } \\
\square & \text { RCRA } \\
\square & \text { TPA\# }\end{array}$ & $\begin{array}{ll} & \text { DELIVERABLE: } \\
\square & \text { Report } \\
\square & \text { Letter } \\
\square & \text { Drawings } \\
\square \text { Other } \\
\text { (specify) }\end{array}$ & 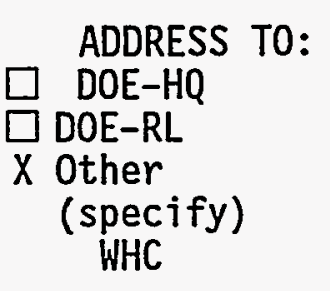 \\
\hline \multicolumn{4}{|l|}{ ilestone Description : } \\
\hline \multicolumn{4}{|c|}{$\begin{array}{l}\text { Assist in preparation of periodic reports to WHC on ICF KH's performance`in } \\
\text { contracting with small disadvantaged, women-owned and labor-surplus area business } \\
\text { concerns. }\end{array}$} \\
\hline \multicolumn{4}{|c|}{$\begin{array}{l}\text { Description of what constitutes completion of this milestone: } \\
\text { Submission of SF } 294 \text { semi-annually. }\end{array}$} \\
\hline $\begin{array}{l}\text { Cost Account Manager } \\
\text { B. E. Hubbard }\end{array}$ & Date & $\begin{array}{l}\text { Program/Project Manager } \\
\text { K. D. Adamson }\end{array}$ & Date \\
\hline $\begin{array}{l}\text { Program Element Manager } \\
\text { K. B. Adamson }\end{array}$ & Date & $\begin{array}{l}\text { DOE Monitor } \\
\text { L. L. Piper }\end{array}$ & Date \\
\hline
\end{tabular}




\section{CONSTRUCTION MANAGEMENT POOL}

HBS 6.5.2

FY 1996 Program Plan

WHC-SP-1122

5.0 COST BASELINE SUMMARY

5.1 Cost Baseline

5.2 Planned Staffing Profile 
ICF Kaiser Hanford

CM Pool

ProposedFY 96 Budget

(In Thousands)

\begin{tabular}{|c|c|c|c|}
\hline & $\begin{array}{c}\text { FY96 } \\
\text { Budget }\end{array}$ & $\begin{array}{l}\text { FY } 95 \\
\text { FYSF }\end{array}$ & $\begin{array}{c}\text { ORIGINAL } \\
\text { FY95 } \\
\text { BUDGET }\end{array}$ \\
\hline Total Labor & 1,215 & 1,735 & 1,484 \\
\hline Nonlaborcosts & 994 * & 1,194 & 1,173 \\
\hline Total Costs & 2,209 & 2,930 & 2,657 \\
\hline FTEs & 30 & 37 & 47 \\
\hline Rate & $2.83 \%$ & $2.92 \%$ & $2.79 \%$ \\
\hline VOLUMEIN MILLIONS & 78 & 100 & 102 \\
\hline
\end{tabular}

* non-laborcosts are not reduced in Fy 96 in relationshipto the reductionsin labor due to the movement of the Hazardous Waste Assessment from within SSPP 6.5 to 6.8, which raised the cost of mangementin the pool by the department overheadadder, and other less significantchanges due to the re-assignment/consolidation. 


\begin{tabular}{|c|c|c|c|c|c|c|c|c|c|c|}
\hline $\begin{array}{l}\text { Work } \\
\text { Breakdown } \\
\text { Structure } \\
\text { Dictionary }\end{array}$ & \multicolumn{6}{|c|}{$\begin{array}{c}\text { Westinghouse Hanford Company } \\
\text { CONTRACT SERVICES } \\
\text { Part I - Summary } \\
\text { (Dollars in 000's) }\end{array}$} & \multicolumn{3}{|c|}{$\begin{array}{l}\text { FY } 1996 \\
\text { Indirect } \\
\text { Program Plain } \\
\text { Rev. \# } 0 \\
20-\text { Sep-95 }\end{array}$} & \\
\hline $\begin{array}{l}\text { Cost Account Number } \\
1 \text { MK3A5 }\end{array}$ & \multicolumn{6}{|c|}{$\begin{array}{l}\text { Cost ACcount Title } \\
\text { CONTRACT SERVICES }\end{array}$} & \multicolumn{3}{|c|}{$\begin{array}{l}\text { Proposed Rate: } \\
\text { (Rated Service Pool Only) }\end{array}$} & \\
\hline $\begin{array}{r}\text { SMS WBS Number } \\
6.5 .2 \\
\end{array}$ & \multicolumn{6}{|c|}{$\begin{array}{l}\text { SMS TItle } \\
\text { CONTAACT SERVICES }\end{array}$} & \multicolumn{3}{|c|}{ Funding Source: } & \\
\hline \multicolumn{7}{|l|}{ RL SMS Program Manger } & \multicolumn{3}{|l|}{ SWs } & \\
\hline Cost Account Manager & \multicolumn{6}{|l|}{ BE HUBBARD } & \multicolumn{3}{|l|}{ OST } & \\
\hline \multicolumn{7}{|c|}{ WHC SMS Program Manager } & & \\
\hline Financial Manager & \multicolumn{6}{|l|}{ MA DAVIS } & \multicolumn{3}{|l|}{ MGT PRO } & \\
\hline Responsible Analyst & \multicolumn{6}{|l|}{ ML VAN LIEW } & \multicolumn{3}{|l|}{ POOL } & \\
\hline & \multicolumn{3}{|c|}{$\begin{array}{c}\text { FY } 1996 \text { Approved Funding } \\
\text { Full-Time Equivalents (FTEs) }\end{array}$} & \multicolumn{3}{|c|}{$\begin{array}{l}\text { FY } 1996 \text { Untunded } \\
\text { Full-Time Equivalents (FTEs) }\end{array}$} & \multicolumn{3}{|c|}{$\begin{array}{c}\text { FY } 1996 \text { Baseline } \\
\text { Full-Time Equivalents (FTEs) } \\
\end{array}$} & \\
\hline TYPE OF FTE & Exempt & Non-Exempt & Bargaining & Exempt & Non-Exempt & Bargaining & Exempt & Non-Exempt & Bargaining & \\
\hline Organizational & 24.5 & 1 & 4 & & & & 24.5 & 1 & 4 & \\
\hline Suppon & 5 & 0.7 & 2.8 & & & & 5 & 0.7 & 2.8 & \\
\hline TOTALFTES & 29.5 & 1.7 & 6.8 & & 0 & 0 & 29.5 & 1.7 & 6.8 & \\
\hline COST ELEMENTS & \multicolumn{3}{|c|}{ FY 1996 Approved Funding Budget } & \multicolumn{3}{|c|}{ FY 1996 Unfunded Budget } & \multicolumn{3}{|c|}{ FY 1996 Baseline Budget } & \\
\hline Labor - Regular & \multicolumn{2}{|c|}{ 1 } & 1214.9 & \multicolumn{3}{|c|}{ 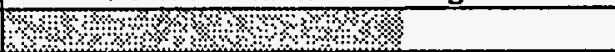 } & \multicolumn{2}{|c|}{ 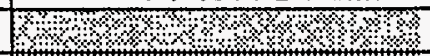 } & 1214.9 & \\
\hline Labor - Overtime & \multicolumn{2}{|c|}{$\% \% ४ \% \% \%$} & 0 & \multicolumn{3}{|c|}{$6 \% \times 1 \% \% \% \%$} & \multicolumn{2}{|c|}{$8140 \% \% 48 \% 3 \% 1 \%$} & & \\
\hline O Total Labor & ४४४४४३ & ४४४४+। & 1214.9 & 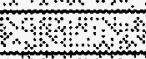 & ৫০। & 0 & ४४। & ४४४४४४७? & 1214.9 & \\
\hline 1 Materials & $1 \% \% \%$ & \$खि? & 69.6 & +1? & ४४०1, & & 1324 & \$४४४४४ & 69.6 & \\
\hline 2 Purchased Services & ८ ४. & 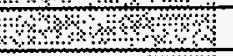 & 154.9 & ४००,० & \$ै०४४ & & ४४०४४ & ४४४४४४४४ & 154.9 & \\
\hline 3 Other Hantord & 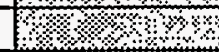 & \%४४४४४। & 0 & 3,6 & 6! ४०। & & $16 \%$ & $4 \%, 4 \%$ & 0 & \\
\hline Sublotal Originated Costs & $18 \%$ & 40,14 & 1439.4 & $14 \%$ & $10.4 \%$ & 0 & $1 \% 4 \%$ & $4.4 \% \%$ & 1439.4 & \\
\hline 4 Site Senices & \% (1. & $6,3,4 \% 4 \%$ & 172 & ४४९ & ४४४,४ ४ & & $848 \%$ & 1./1\% ४ & 172 & \\
\hline 5 Internal Charges & $\%$ & $1+\%$ & 127 & $\%$ & ४४४४४४ & & 14,8 & $10 \% 8 \%$ & 127 & \\
\hline 6 IRM Support & \% \% \% & $3,3 \%$ & 149.8 & ।ै। & ४४४४। & & 184 & $4 \%, 2 \%$ & 149.8 & \\
\hline 7 Overheads & $3 \%, 2,2$ & $\%(\%, ४)$ & 321 & 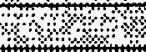 & $3 \%,+18 \%$ & & 8) & $4.8 \% 1 \% 14$ & 321 & \\
\hline 8 Revenue & 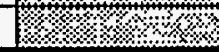 & $30<\%<\%$ & 0 & 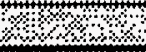 & 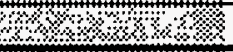 & & $3<\% \%$ & $14 \div 6 \%$ & & \\
\hline TOTAL DOLLARS & W & К\% ะ & 2209.2 & $8 \% 8 \%$ & ४४ $+2 \%$ & & $\sqrt{8 \% 2 \% 8 \% ~}$ & 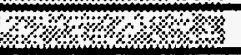 & 2209.2 & \\
\hline Budget EXCLUDES the ce & of direct labor a & and job shoppers. & s. $(\$ 1422.7 \mathrm{~K}) \mathrm{He}$ & adcount & & & & & & \\
\hline $\begin{array}{l}\text { SIGNATURES } \\
\text { Financial Analyst: MoK } \\
\text { CAM: }\end{array}$ & comotas & if & Date: 962965 & & BASELINE APF & PROVAL & & & Date: & \\
\hline
\end{tabular}




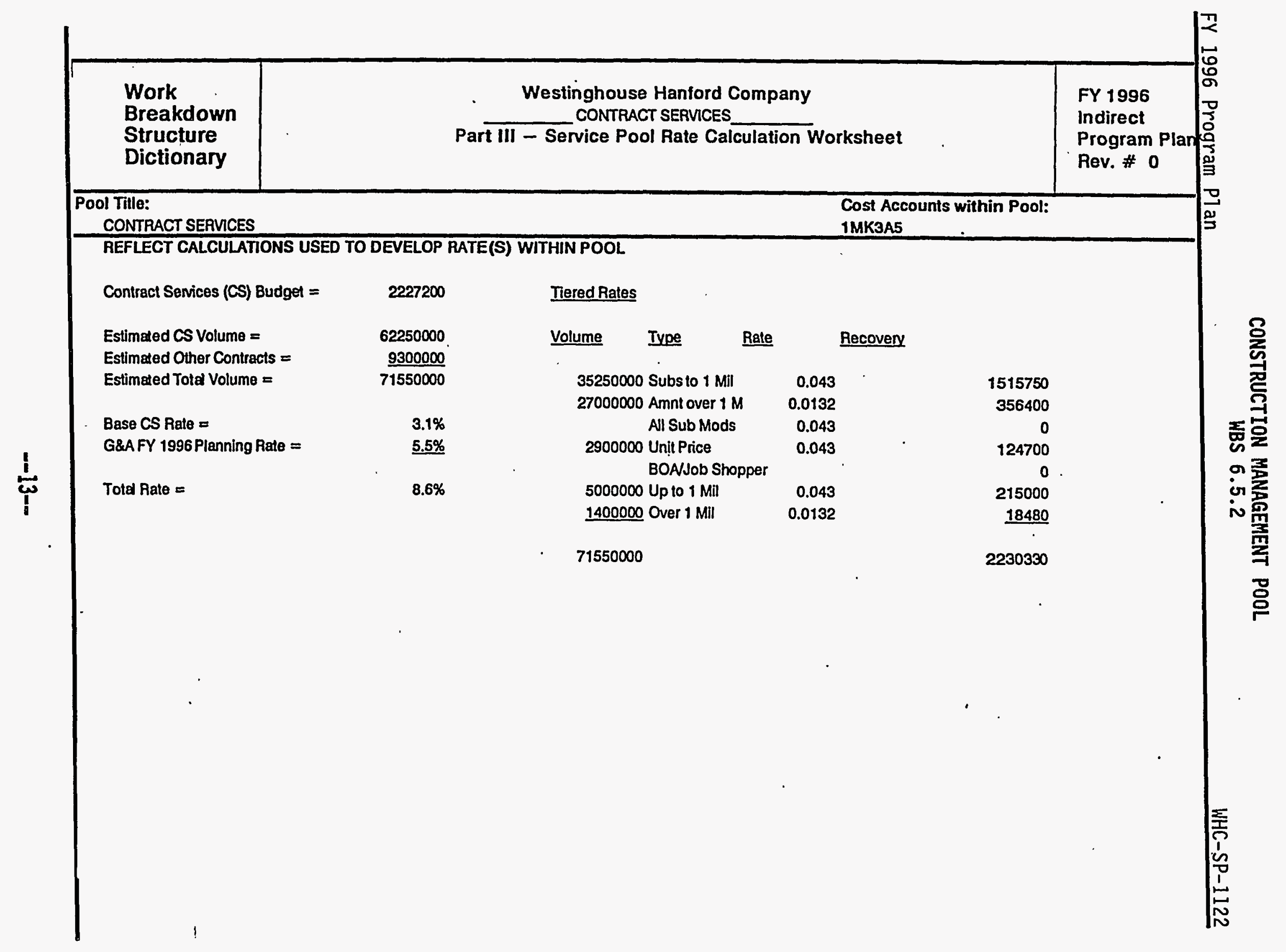




\begin{tabular}{|c|c|c|c|c|c|c|c|c|c|}
\hline \multicolumn{4}{|c|}{ Planned Staffing (Full Time Equivalent) } & \multicolumn{5}{|c|}{ Note: Job Family Only After 1997} & \\
\hline Job Fam $1 \mathrm{y}$ - & Number: & m. & \%थ & अß & मे. & ॠै। & ४ै। & \& & \\
\hline Job Category & & 1996 & 1997 & 1998 & 1999 & 2000 & 2001 & 2002 & \\
\hline MANAGERS & २०० & खे & +ै? & अ1: & +1? & अे। & अे\% & +1 & \\
\hline First line & 1 & 1 & 1 & & & & & & \\
\hline \multicolumn{9}{|l|}{ General/executive } & \\
\hline \multicolumn{9}{|l|}{ Projec/Program } & \\
\hline \multicolumn{9}{|l|}{ other } & \\
\hline \multicolumn{9}{|l|}{ Subtotal } & \\
\hline ENGINEERS $\%$ \%े? & (2) $\%$ & 2 & ঔ২২े & 3. & $\% 3 \%$ & ?. 3. & +3? & 3 & \\
\hline \multicolumn{9}{|c|}{\begin{tabular}{|l|l|l|l|l|l|} 
Chemical & & & & & \\
\end{tabular}} & \\
\hline Civil & 3 & 3 & 3 & & & & & & \\
\hline \multicolumn{9}{|l|}{ Computer } & \\
\hline \multicolumn{9}{|l|}{ Electrical } & \\
\hline \multicolumn{9}{|l|}{ Environmental } & \\
\hline \multicolumn{9}{|l|}{ Industrial } & \\
\hline \multicolumn{9}{|l|}{ Mechanical } & \\
\hline \multicolumn{9}{|l|}{ Nuclear } & \\
\hline \multicolumn{9}{|l|}{ Petroleum/Mining } & \\
\hline \multicolumn{9}{|l|}{ Plant } & \\
\hline \multicolumn{9}{|l|}{ Quality Control } & \\
\hline \multicolumn{9}{|l|}{ Safety } & \\
\hline \multicolumn{9}{|l|}{ other } & \\
\hline Subtotal & & & & & & & & & \\
\hline
\end{tabular}




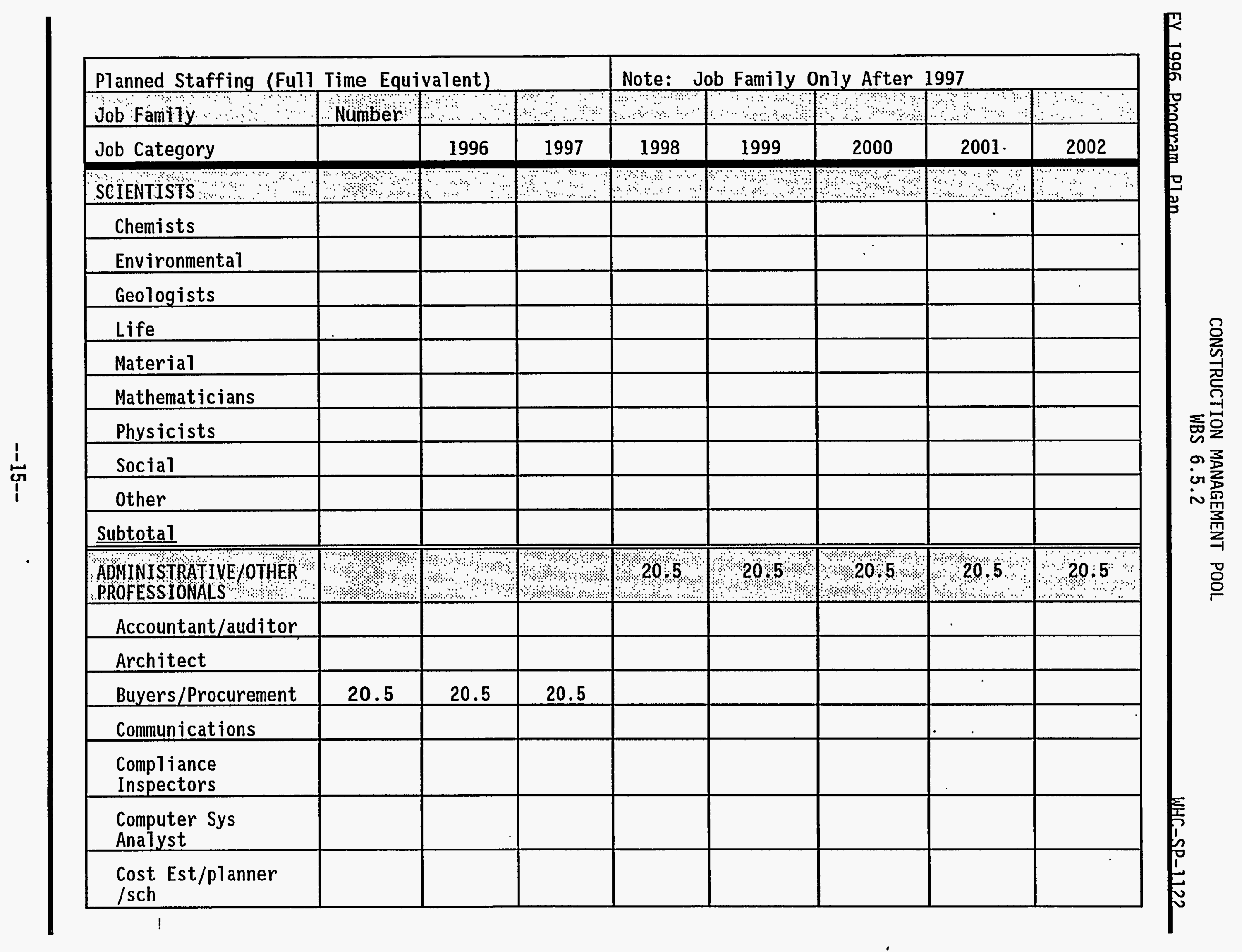




\begin{tabular}{|c|c|c|c|c|c|c|c|c|}
\hline \multicolumn{4}{|c|}{ Planned Staffing (Full Time Equivalent) } & \multicolumn{5}{|c|}{ Note: Job Family Only After 1997} \\
\hline Job: Family & Number: & \$\%. & यद्य & औस. & \%स & ४थי & \%...? & 14\% \\
\hline Job Category & & 1996 & 1997 & 1998 & 1999. & 2000 & 2001 & 2002 \\
\hline \multicolumn{9}{|l|}{ Health Physics } \\
\hline \multicolumn{9}{|l|}{ Industrial' Hygiene } \\
\hline \multicolumn{9}{|l|}{ Lawyers } \\
\hline \multicolumn{9}{|l|}{$\begin{array}{l}\text { Personne } 1 / 1 \text { abor } \\
\text { Relations }\end{array}$} \\
\hline \multicolumn{9}{|l|}{ Physicians } \\
\hline \multicolumn{9}{|l|}{$\begin{array}{l}\text { Physician } \\
\text { Assistant/Nurse }\end{array}$} \\
\hline \multicolumn{9}{|l|}{$\begin{array}{l}\text { Safeguard \& } \\
\text { Security }\end{array}$} \\
\hline \multicolumn{9}{|l|}{$\begin{array}{l}\text { Tech Writers \& } \\
\text { Editors }\end{array}$} \\
\hline \multicolumn{9}{|l|}{ Trainers } \\
\hline \multicolumn{9}{|l|}{ Other } \\
\hline \multicolumn{9}{|l|}{ Subtotal } \\
\hline $\begin{array}{l}\text { GENERALECRETARY:GLERK } \\
\text { AOMIN/SECRE }\end{array}$ & क०. & +4 & $2+$ & 15 & 5 & -5 & 5 & 5 \\
\hline \multicolumn{9}{|l|}{ Admin Assistant } \\
\hline Office Clerk (Gen) & 3 & 3 & 3 & & & & & \\
\hline \multicolumn{9}{|l|}{$\begin{array}{l}\text { Office Clerk } \\
\text { (Special) }\end{array}$} \\
\hline Secretaries & 2 & 2 & 2 & & & & & \\
\hline \multicolumn{9}{|l|}{$\begin{array}{l}\text { Typist/Word } \\
\text { Processor }\end{array}$} \\
\hline Other & & & & & & & & \\
\hline
\end{tabular}




\begin{tabular}{|c|c|c|c|c|c|c|c|c|}
\hline \multirow{2}{*}{\multicolumn{9}{|c|}{\begin{tabular}{l|l} 
Planned Staffing (Ful1 Time Equivalent) & Note: Job Family only After 1997 \\
\end{tabular}}} \\
\hline Job Famuly $* \cdots$ & Nümber: & $2 \%$ & मे: & a & ४ै। & अः & से & \\
\hline Job Category & . & 1996 & 1997 & 1998 & 1999 & 2000 & 2001 & 2002 \\
\hline \multicolumn{9}{|l|}{ Subtotal } \\
\hline TECHNICIANS & अै। & कि & अं। & ans & बिए & अै। & अे, & 8. \\
\hline \multicolumn{9}{|l|}{$\begin{array}{l}\text { Computer } \\
\text { Operator/Coder }\end{array}$} \\
\hline \multicolumn{9}{|l|}{ Drafters } \\
\hline \multicolumn{9}{|l|}{ Engineers/Technicians } \\
\hline \multicolumn{9}{|l|}{$\begin{array}{l}\text { Environmental } \\
\text { Science } \\
\text { Technicians }\end{array}$} \\
\hline \multicolumn{9}{|l|}{$\begin{array}{l}\text { Health Physics } \\
\text { Technicians }\end{array}$} \\
\hline \multicolumn{9}{|l|}{$\begin{array}{l}\text { Industrial } \\
\text { Saf/health } \\
\text { Technicians }\end{array}$} \\
\hline \multicolumn{9}{|l|}{$\begin{array}{l}\text { Instru/Control } \\
\text { Technicians. }\end{array}$} \\
\hline \multicolumn{9}{|l|}{ Lab. Technicians } \\
\hline \multicolumn{9}{|l|}{ Media Technicians } \\
\hline \multicolumn{9}{|l|}{$\begin{array}{l}\text { Survey/Map } \\
\text { Technicians }\end{array}$} \\
\hline \multicolumn{9}{|l|}{ other } \\
\hline \multicolumn{9}{|l|}{ Subtotal } \\
\hline 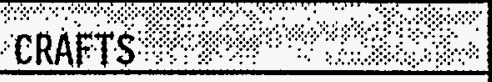 & $\% \%$ & юथ & ४ै\%? & ४०० & अి ४ै & ४ै।. & \&. & \\
\hline Carpenters & & $\cdot$ & & & & & & \\
\hline
\end{tabular}




\begin{tabular}{|c|c|c|c|c|c|c|c|c|}
\hline \multicolumn{4}{|c|}{ Planned Staffing (Full Time Equivalent) } & \multicolumn{5}{|c|}{ Note: Job Family Only After 1997} \\
\hline Job Family : el & Number & अं। & अ? & बि & थे & ڤ४० & ४ै। & 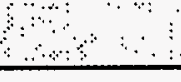 \\
\hline Job Category & & 1996 & 1997 & 1998 & 1999 & 2000 & 2001 & 2002 \\
\hline \multicolumn{9}{|l|}{ Electricians } \\
\hline \multicolumn{9}{|l|}{ HVAC } \\
\hline \multicolumn{9}{|l|}{ Machinists } \\
\hline \multicolumn{9}{|l|}{ Masons } \\
\hline \multicolumn{9}{|l|}{ Millwrights } \\
\hline \multicolumn{9}{|l|}{ Painters } \\
\hline \multicolumn{9}{|l|}{ Plmbrs/Pipefitters } \\
\hline \multicolumn{9}{|l|}{$\begin{array}{l}\text { Struct/Metal } \\
\text { Workers }\end{array}$} \\
\hline \multicolumn{9}{|l|}{$\begin{array}{l}\text { Vehicl/Mobile } \\
\text { Equip. } \\
\text { Mechanics }\end{array}$} \\
\hline \multicolumn{9}{|l|}{ Welders } \\
\hline \multicolumn{9}{|l|}{ Other } \\
\hline \multicolumn{9}{|l|}{ Subtotal } \\
\hline OPERATORS & ४०० & अ» & ঋ४। & अ० & अै। & अै? & ४ै: & में \\
\hline \multicolumn{9}{|l|}{ Lt. Vehicle Drivers } \\
\hline \multicolumn{9}{|l|}{$\begin{array}{l}\text { Material Moving } \\
\text { Equip. }\end{array}$} \\
\hline \multicolumn{9}{|l|}{ Nuclear Plant } \\
\hline \multicolumn{9}{|l|}{$\begin{array}{l}\text { Utilities Waste } \\
\text { Proces }\end{array}$} \\
\hline \multicolumn{9}{|l|}{ Other } \\
\hline Subtotal & & & & & & & & \\
\hline
\end{tabular}




\begin{tabular}{|c|c|c|c|c|c|c|c|c|}
\hline \multicolumn{4}{|c|}{ Planned Staffing (Full Time Equivalent) } & \multicolumn{5}{|c|}{ Note: Job Family Only After 1997} \\
\hline Job Fam $1 \mathrm{y}, \mathrm{a}$ & Number: & 2 & $\$$ & $\begin{array}{r}3+2 \\
\end{array}$ & $\because \quad$ & +? & 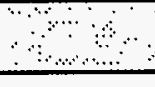 & $\therefore \quad \cdots$ \\
\hline Job Category & & 1996 & 1997 & 1998 & 1999 & 2000 & 2001 & 2002 \\
\hline $\begin{array}{l}\text { CABOR \& GENERAL } \\
\text { WORKERS }\end{array}$ & अै। & \%s & अे। & क्ष & मे & ڤ। & 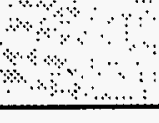 & ras \\
\hline Firefighters & & & & & & & & \\
\hline Food Service & & & & & & & & \\
\hline Hand/Help Lab Gen & & & & & & & & \\
\hline $\begin{array}{l}\text { Hand/Heip Lab } \\
\text { Special }\end{array}$ & & & & & & & & \\
\hline Janitors/Cleaners & & & & & & & & \\
\hline Laundry Workers & & & & & & & & \\
\hline Security Guards & & & & & & & & \\
\hline Other & & & & & & & & \\
\hline Subtotal & & & & & & & & \\
\hline TOTAL FTES & & & & & & & & \\
\hline
\end{tabular}




\section{INDEX}

1.0 CONSTRUCTION FORCES POOL

2.0 CONSTRUCTION FORCES OVERVIEW (WBS 6.5.3)

2.1 Mission

2.2 Assumptions

2.3 Constraints

3.0 TECHNICAL REQUIREMENT BASELINE

3.1 Work Breakdown Structure

3.2 Work Breakdown

3.1.1 Construction Services (WBS 6.5.3.1)

3.1.2 TWRS (WBS 6.5.3.2)

3.1.3 SNF (WBS 6.5.3.3)

3.1.4 PNL Laridlord, Engineering and Construction Services (WBS 6.5.3.4)

3.1.5 Transition Projects/Site Engineering and Construction Services (WBS 6.5.3.5)

4.0 SCHEDULE BASELINE

4.1 Product Deliverables/Milestone List

5.0 COST BASELINE SUMMARY

5.1 Cost Baseline

5.2 Planned Staffing Profile 


\subsection{CONSTRUCTION FORCES POOL}

The Construction Forces (CF) provides crafts training, radiation protection, fabrication shop, and hazardous waste assessment. Construction Forces management assures safety through quality control inspection and industrial hygiene.

\subsection{CONSTRUCTION FORCES OVERVIEW (WBS 6.5.3)}

\subsection{Mission}

Construction Forces: Ensures that all functions are accomplished in support of the construction role.

Environmental Safety and Health (ES\&H): Educate management and employees to protect personnel and the environment.

Note: To accomplish this mission, the Construction Forces personnel have been assigned to support various ICF KH organizations.

\subsection{Assumptions}

The following are key assumptions used to develop goals and strategic (building blocks): Reduction of cost is paramount to success and competitive posturing. Outsourcing of work will be conducted on a cost/performance advantage basis, funding and staffing levels will be adequate, re-engineering initiatives will be fully supported, award fee and incentive fee criteria will be established, cost savings programs will be supported, work will comply with current regulations and guidance, and the Voluntary Protection Program will continue.

\subsection{Constraints}

Items that may impede achievements of goals and strategies are as follows: privatization and outsourcing of services, funding support to ICF KH must be adequate for the scope required by DOE, contradictory DOE missions, scheduling/cost software programs changed, prioritization of work due to budget constraints, and continued company reorganizations. 


\subsection{TECHNICAL REQUIREMENTS BASELINE}

\subsection{Work Breakdown Structure}

3.2 Work Breakdown 


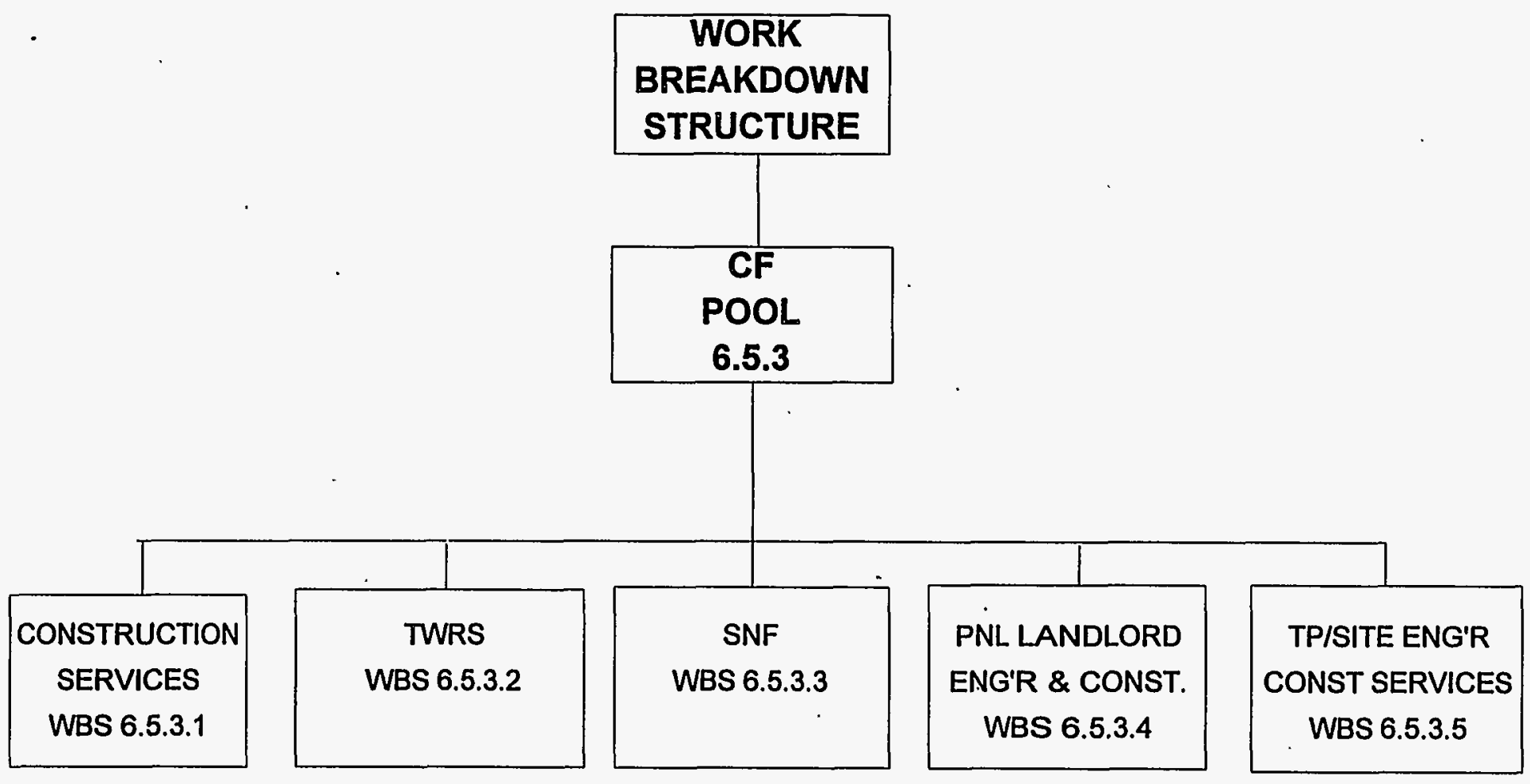




\section{ONSTRUCTION FORCES POOL (6.5.3)}

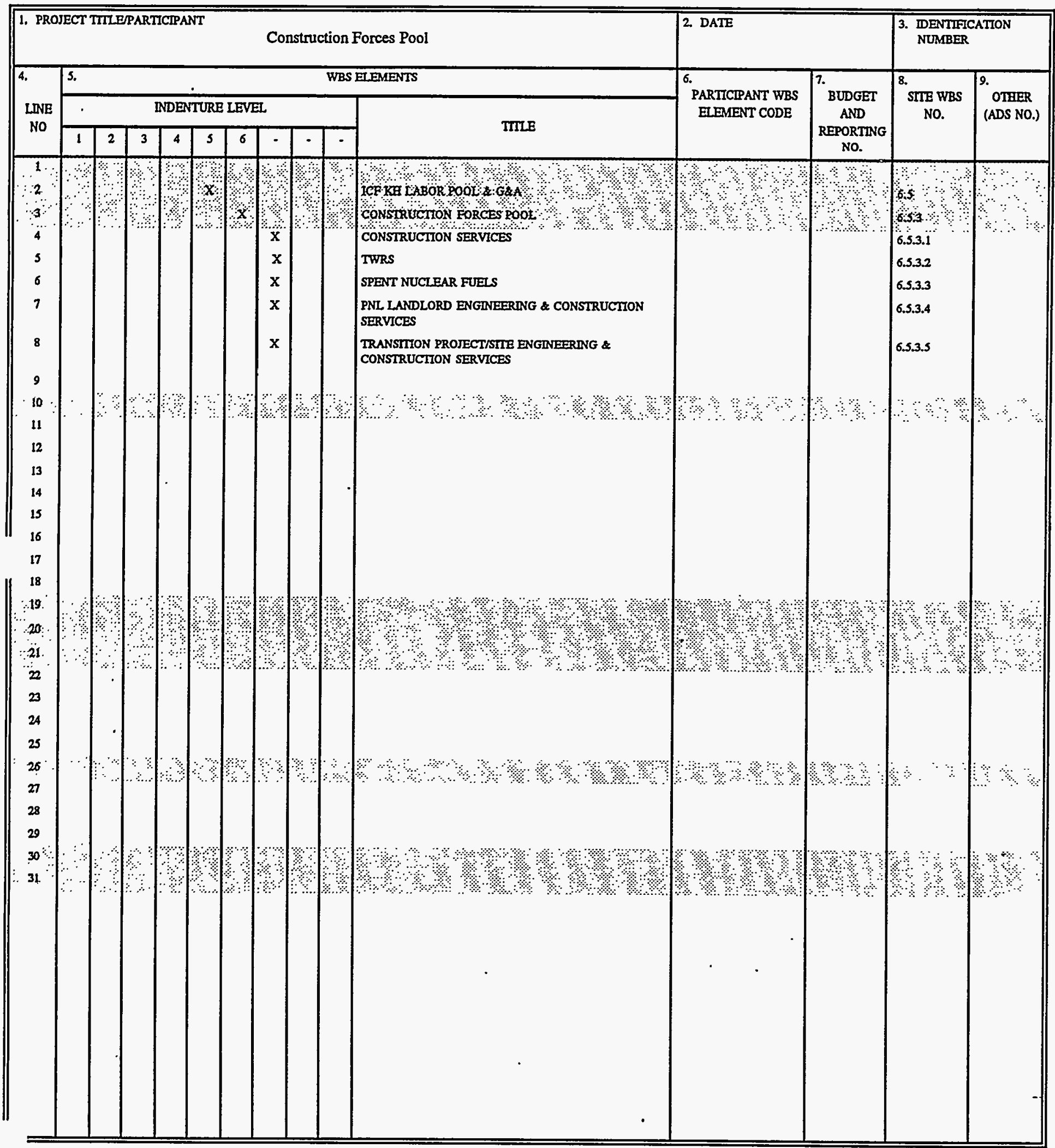


FY 1996 Program P1 an

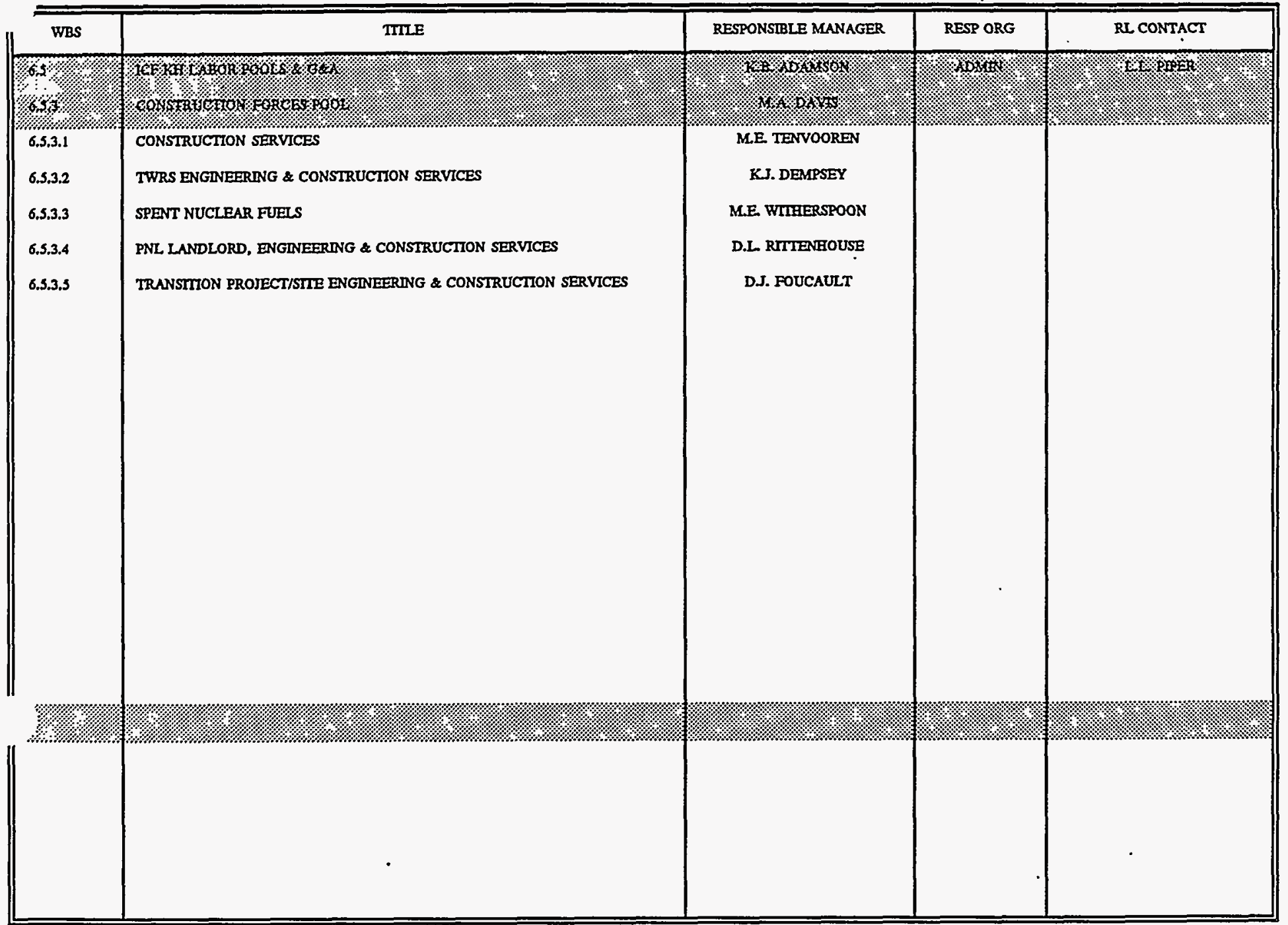


Table 3-1. FY 1996 SSPP CONSTRUCTION FORCES POOL WBS Dictionary (6.5.3)

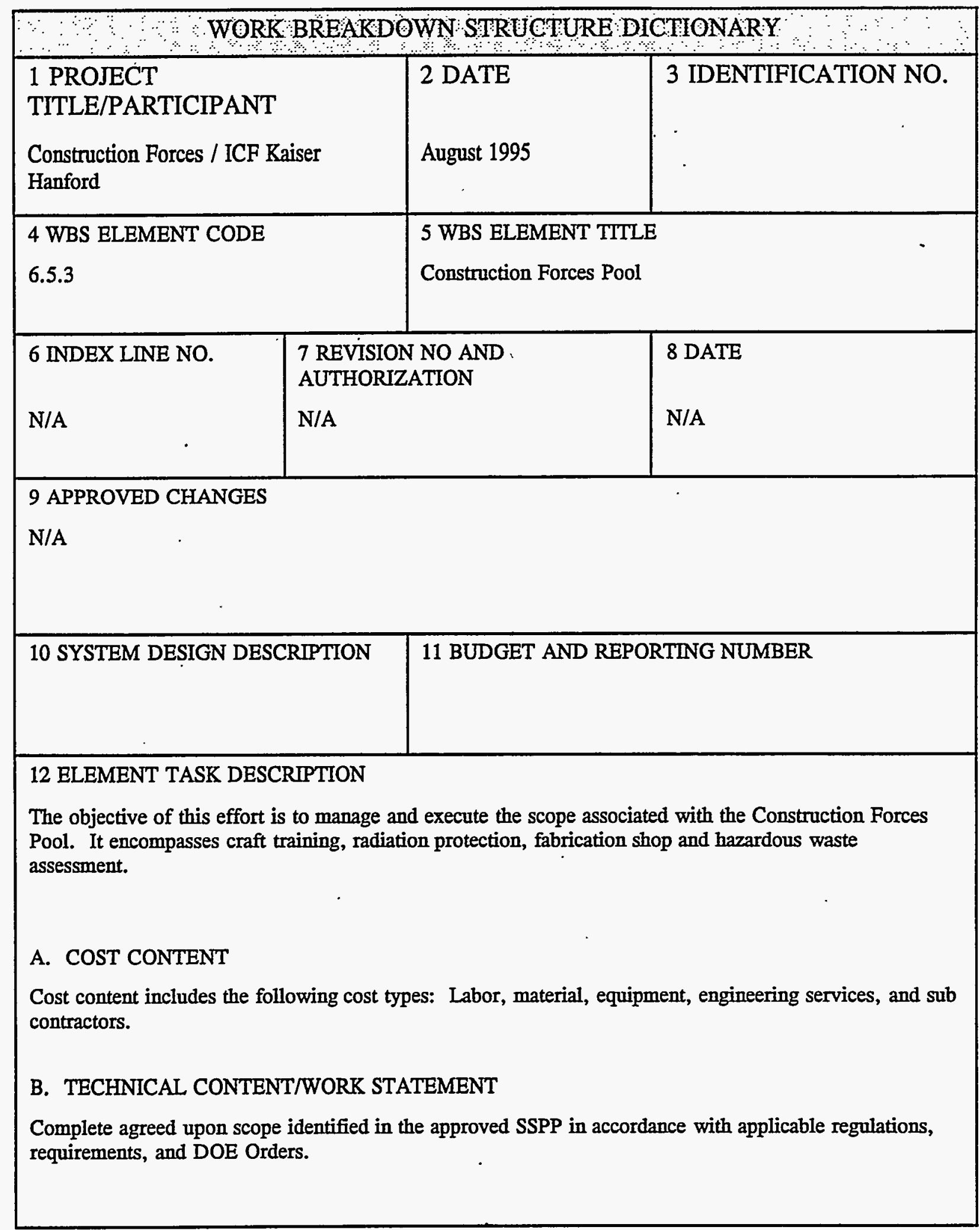




\subsubsection{Construction Services (WBS 6.5.3.1)}

\section{Mission}

The Construction Services Division sets standards and practices related to construction activities to ensure safety and consistency. The standards and practices are utilized by each organization that performs construction activities.

\section{Products/Services Provided}

The Construction Services Division provides the following: construction safety and health programs, processes for corrective action planning in the construction divisions, training programs for construction and evaluate ergonomics for construction related activities. Also includes ASME Code Engineer Services, Quality Control surveillance activities, NEC Interpretive Authority and related procedure development and control.

\section{Primary Customers}

The primary customer of the Construction Services Division are: ICF Kaiser Hanford, Westinghouse Hanford Company, Pacific Northwest Laboratory, and the U.S. Department of Energy (RL). 


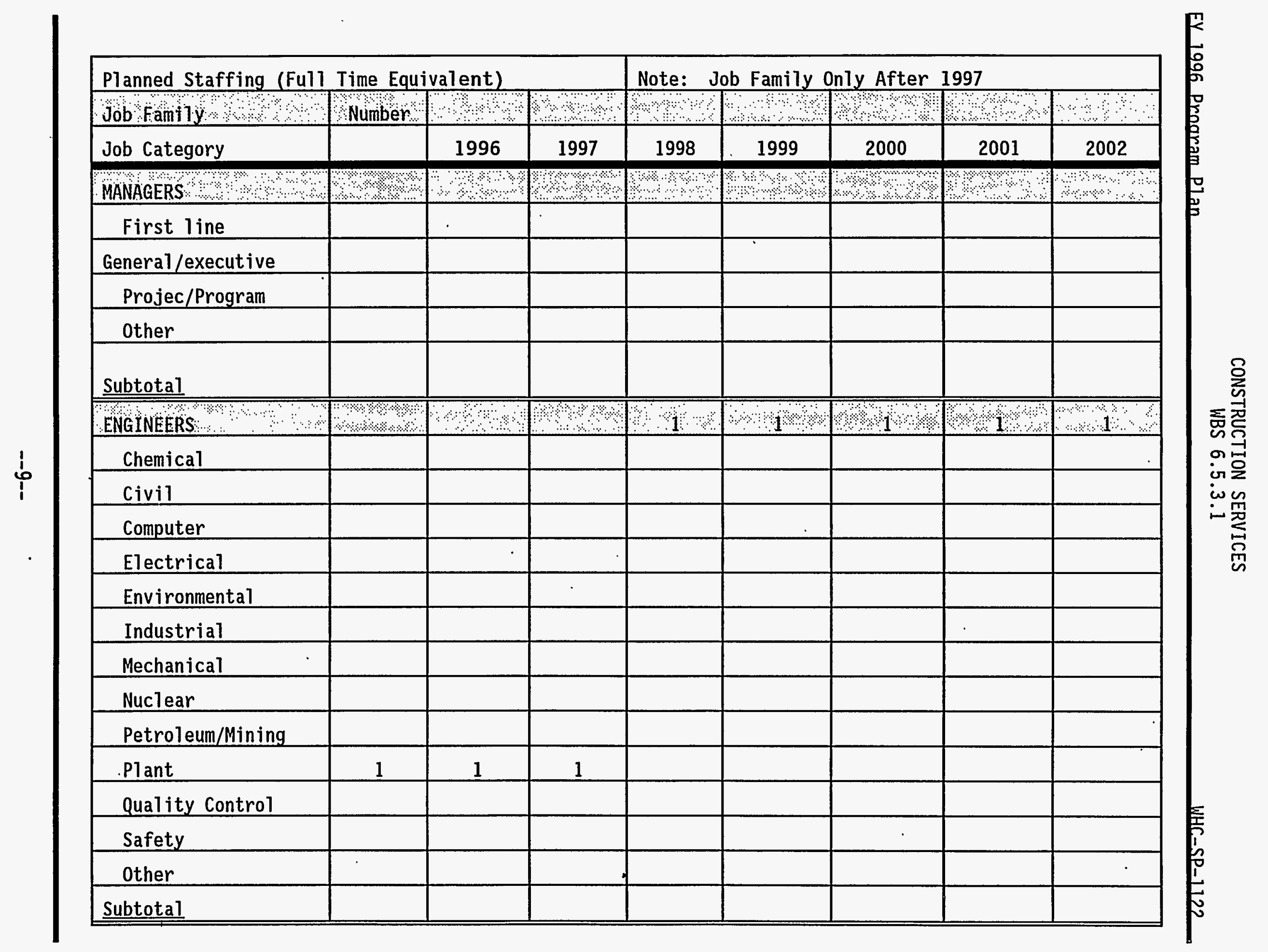




\begin{tabular}{|c|c|c|c|c|c|c|c|c|}
\hline \multicolumn{4}{|c|}{ Planned Staffing (Full Time Equivalent) } & \multicolumn{5}{|c|}{ Note: Job Family Only After 1997} \\
\hline Job Family & Number & ins & अे० & +ి & ४ै। & ४ै। & ঋै। & +४। \\
\hline Job Category & & 1996 & 1997 & 1998 & 1999 & 2000 & 2001 & 2002 \\
\hline SCIENTISTS & ४४ ४ & ४४ : & अ४४ & ২४৮ & ४४९ & 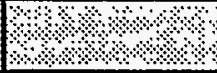 & 1 & ४४ \\
\hline Chemists & & & & & & & & \\
\hline Environmental & & & & & & & & \\
\hline Geologists & & & & & & & & \\
\hline Life & & & & & & & & \\
\hline Material & & & & & & & & \\
\hline Mathematicians & & & & & & & & \\
\hline Physicists & & & & & & & & \\
\hline Social & & & & & & & & \\
\hline Other & & & & & & & & \\
\hline Subtotal & & & & & & & & \\
\hline $\begin{array}{l}\text { ADMINISTRATIVE OTHER } \\
\text { PROEESSTONALS }\end{array}$ & $2 \%$ & \%े। & \%ै। & धै। & अै? & ?ै? & \%? & िै। \\
\hline Accountant/auditor & & & & & & & & \\
\hline Architect & & & & & & & & \\
\hline Buyers/Procurement & & & & & & & & \\
\hline Communications & & & & & & . & & \\
\hline $\begin{array}{l}\text { Comp } 1 \text { iance } \\
\text { Inspectors }\end{array}$ & & & & & & & & \\
\hline $\begin{array}{l}\text { Computer Sys } \\
\text { Analyst }\end{array}$ & & & & & & & & \\
\hline $\begin{array}{l}\text { Cost Est/planner } \\
\text { /sch }\end{array}$ & & & & & & & & \\
\hline
\end{tabular}




\begin{tabular}{|c|c|c|c|c|c|c|c|c|}
\hline \multicolumn{4}{|c|}{ Planned Staffing (Full Time Equivalent) } & \multicolumn{5}{|c|}{ Note: Job Family Only After 1997} \\
\hline Job Family & Number & \%४ि & ४० & अध & ख४ & अ४? & ४० & अ४ \\
\hline Job Category & & 1996 & 1997 & 1998 & 1999 & 2000 & 2001 & 2002 \\
\hline \multicolumn{9}{|l|}{ Health Physics } \\
\hline \multicolumn{9}{|l|}{ Industrial Hygiene } \\
\hline \multicolumn{9}{|l|}{ Lawyers } \\
\hline \multicolumn{9}{|l|}{$\begin{array}{l}\text { Personnel/labor } \\
\text { Relations }\end{array}$} \\
\hline \multicolumn{9}{|l|}{ Physicians } \\
\hline \multicolumn{9}{|l|}{$\begin{array}{l}\text { Physician } \\
\text { Assistant/Nurse }\end{array}$} \\
\hline \multicolumn{9}{|l|}{$\begin{array}{l}\text { Safeguard \& } \\
\text { Security }\end{array}$} \\
\hline \multicolumn{9}{|l|}{$\begin{array}{l}\text { Tech Writers \& } \\
\text { Editors }\end{array}$} \\
\hline \multicolumn{9}{|l|}{ Trainers } \\
\hline \multicolumn{9}{|l|}{ other } \\
\hline \multicolumn{9}{|l|}{ Subtotal } \\
\hline $\begin{array}{l}\text { GENERAL/SER } \\
\text { ADMIN/SECRETARY/GLERK }\end{array}$ & \%? & अ? & $2 \%$ & कि & मिः & ? & +1. & 1 \\
\hline Admin Assistant & & & & & & & & . \\
\hline Office Clerk (Gen) & 1 & 1 & 1 & & & & & \\
\hline \multicolumn{9}{|l|}{$\begin{array}{l}\text { Office Clerk } \\
\text { (Special) }\end{array}$} \\
\hline \multicolumn{9}{|l|}{ Secretaries } \\
\hline \multicolumn{9}{|l|}{$\begin{array}{l}\text { Typist/Word } \\
\text { Processor }\end{array}$} \\
\hline other & & & & & & & & \\
\hline
\end{tabular}




\begin{tabular}{|c|c|c|c|c|c|c|c|c|}
\hline \multicolumn{4}{|c|}{ Planned Staffing (Ful1 Time Equivalent) } & \multicolumn{5}{|c|}{ Note: Job Family Only After 1997} \\
\hline Job Famliy & Number: & +ि & ४ै & ४ & : & $\$$ & «) & बे \\
\hline Job Category & & 1996 & 1997 & 1998 & 1999 & 2000 & 2001 & 2002 \\
\hline Subtotal & & & & & & & & \\
\hline TECHNIC IANS & ४ै। : & अेक & ४०ै & अ9. & २े 9. & अ४. $9 \%$ & अ 9 & 9 \\
\hline $\begin{array}{l}\text { Computer } \\
\text { Operator/Coder }\end{array}$ & & . & & & & & & \\
\hline Drafters & & & & & & & & \\
\hline Engineers/Technicians & 9 & 9 & 9 & & & & & \\
\hline $\begin{array}{l}\text { Environmental } \\
\text { Science } \\
\text { Technicians }\end{array}$ & & & & & & & & \\
\hline $\begin{array}{l}\text { Health Physics } \\
\text { Technicians }\end{array}$ & & & & & & & & \\
\hline $\begin{array}{l}\text { Industrial } \\
\text { Saf/health } \\
\text { Technicians }\end{array}$ & & & & & & & & \\
\hline $\begin{array}{l}\text { Instru/Control } \\
\text { Technicians }\end{array}$ & & & & & & & & \\
\hline Lab. Technicians & & & & & & & & \\
\hline Media Technicians & & & & & & & & \\
\hline $\begin{array}{l}\text { Survey/Map } \\
\text { Technicians }\end{array}$ & & & & & & & & \\
\hline Other & & & & & & & & \\
\hline Subtotal & & & & & & & & \\
\hline GRAETS $\%$ ४ \% & अथे & $\%$ & थै। & ४४ & ४ै\% & ४े & ए. & \\
\hline Carpenters & & & & & & & & \\
\hline Electricians & & & & & & & & \\
\hline
\end{tabular}




\begin{tabular}{|c|c|c|c|c|c|c|c|c|}
\hline \multicolumn{4}{|c|}{ Planned Staffing (Full Time Equivalent) } & \multicolumn{5}{|c|}{ Note: Job Family Only After 1997} \\
\hline Job Family & Number: & अ & «ি: & - य & ॥. & ४ै ४ै। & अ : & बथ \\
\hline Job Category & & 1996 & 1997 & 1998 & 1999 & 2000 & 2001 & 2002 \\
\hline \multicolumn{9}{|l|}{ HVAC } \\
\hline \multicolumn{9}{|l|}{ Machinists } \\
\hline \multicolumn{9}{|l|}{ Masons } \\
\hline \multicolumn{9}{|l|}{ Millwrights } \\
\hline \multicolumn{9}{|l|}{ Painters } \\
\hline \multicolumn{9}{|l|}{ Plmbrs/Pipefitters } \\
\hline \multicolumn{9}{|l|}{$\begin{array}{l}\text { Struct/Metal } \\
\text { Workers }\end{array}$} \\
\hline \multicolumn{9}{|l|}{$\begin{array}{l}\text { Vehici/Mobile } \\
\text { Equip. } \\
\text { Mechanics. }\end{array}$} \\
\hline \multicolumn{9}{|l|}{ Welders } \\
\hline \multicolumn{9}{|l|}{ other } \\
\hline \multicolumn{9}{|l|}{ Subtotal } \\
\hline OPERATORS & ৩২े। & १४४४ & \%े & के: & \%०े। & १४ै। & और & 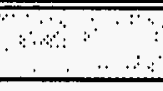 \\
\hline \multicolumn{9}{|l|}{ Lt. Vehicle Drivers } \\
\hline \multicolumn{9}{|l|}{$\begin{array}{l}\text { Material Moving } \\
\text { Equip. }\end{array}$} \\
\hline \multicolumn{9}{|l|}{ Nuclear Plant } \\
\hline \multicolumn{9}{|l|}{$\begin{array}{l}\text { Utilities Waste } \\
\text { Proces }\end{array}$} \\
\hline \multicolumn{9}{|l|}{ Other } \\
\hline Subtotal & & & & & & & & \\
\hline
\end{tabular}




\begin{tabular}{|c|c|c|c|c|c|c|c|c|}
\hline \multicolumn{4}{|c|}{ Planned Staffing (Full Time Equivalent) } & \multicolumn{5}{|c|}{ Note: Job Family Only After 1997} \\
\hline Job Famtly & Number: & wa & \%० & * & \%के & ४ै० & +e & is \\
\hline Job Category & & 1996 & 1997 & 1998 & 1999 & 2000 & 2001 & 2002 \\
\hline $\begin{array}{l}\text { LABOR \& GENERAL } \\
\text { WORKERS }\end{array}$ & + & 4 & अ? & ४ै। & \%ै। & +4. & ४ै। & s: \\
\hline Firefighters & & & & & & & & \\
\hline Food Service & & & & & & & & \\
\hline Hand/Help Lab Gen & & & & & & & & \\
\hline $\begin{array}{l}\text { Hand/Help Lab } \\
\text { Special }\end{array}$ & & & & & & & & \\
\hline Janitors/Cleaners & & & & & & & & \\
\hline Laundry Workers & & & & & & & & \\
\hline Security Guards & & & & & & & & \\
\hline Other & & & & & & & & \\
\hline$\underline{\underline{\underline{\text { Subtotal }}}}$ & & & & & & & & \\
\hline IOTAL FTES & & & & & & & & \\
\hline
\end{tabular}


3.1.2 Tank Waste Remediation System (TWRS) Engineering \& Construction Services (WBS 6.5.3.2)

\section{Mission}

The TWRS Engineering and Construction Services Division, in its capacity as the functional project management entity, has the responsibility for the supervision and support of the Construction Forces and ES\&H activities associated with the Division.

\section{Product/Services Provided}

The TWRS Engineering and Construction Services Division provides the following: Liaison between ICF KH and WHC projects personnel and plant operators for CF construction activities coordination; Construction supervision, engineering and scheduling; support technical expertise and recommendations to assist in the solution of problems encountered prior to or during construction in the field; safety walkdowns and inspections; input to corrective actions resulting for audits, surveillances and accidents; Kaiser Injury Prevention System (KIPS) database; procedures to implement requirements of WHC-CM-7-5 consistent7y throughout ICF KH; input to the ICF KH portion of the Hanford Annual Dangerous Waste Report.

\section{Primary Customer}

The primary customer of the TWRS Engineering and Construction Services Division are: ICF Kaiser Hanford, Westinghouse Hanford Company, and the U.S. Department of Energy (RL). 


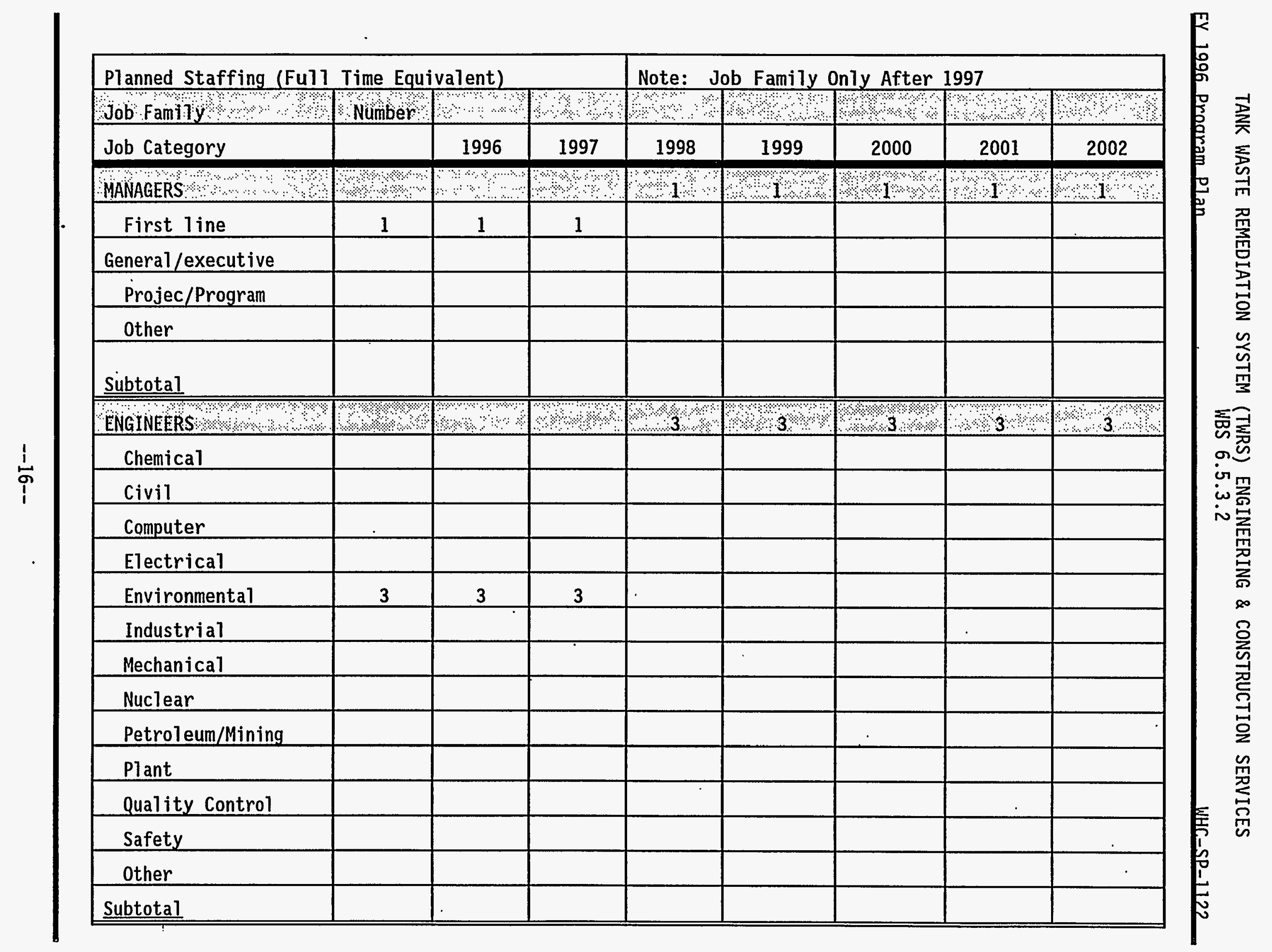




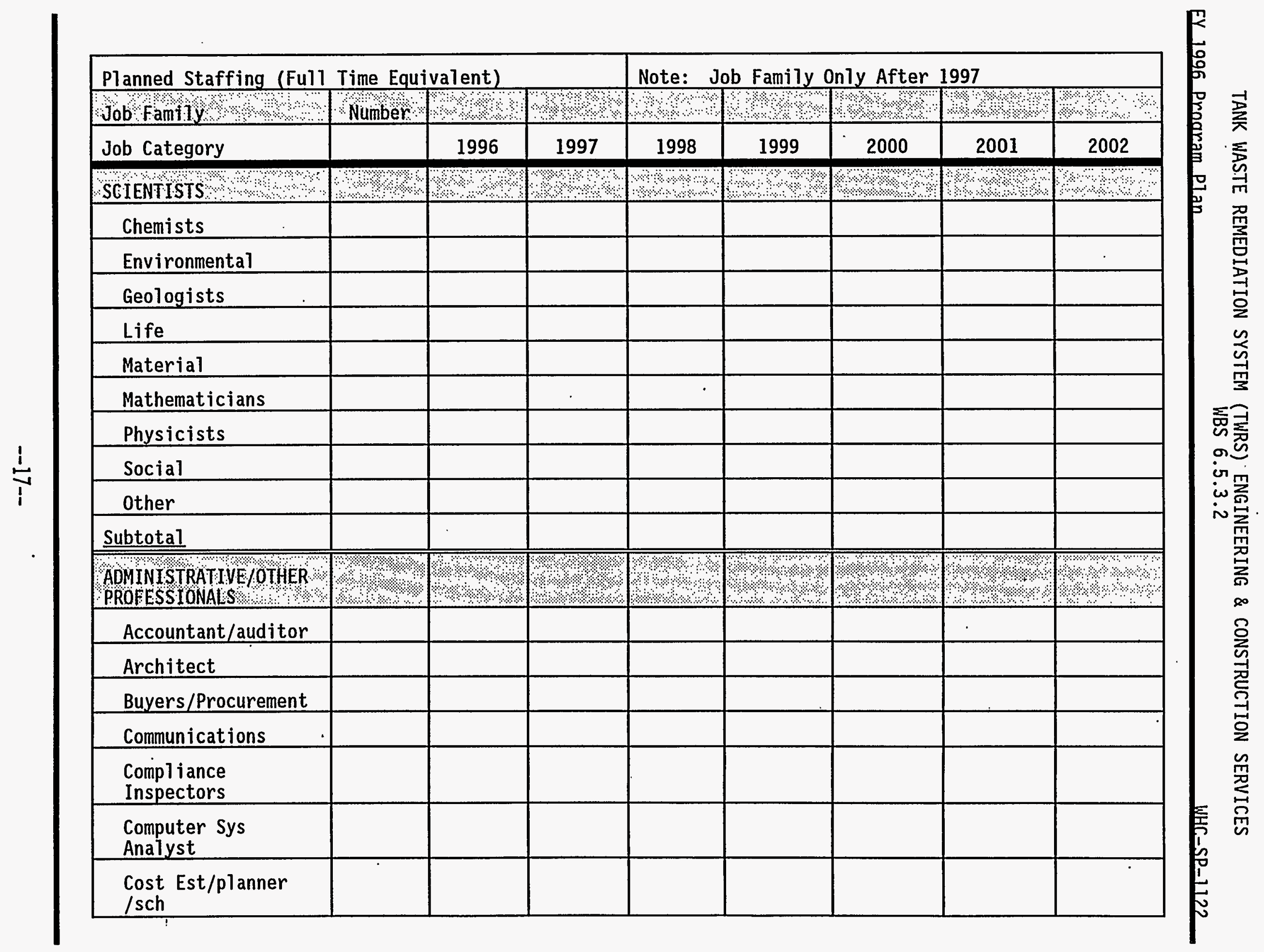




\begin{tabular}{|c|c|c|c|c|c|c|c|c|}
\hline \multicolumn{4}{|c|}{ Planned Staffing (Fu11 Time Equivalent) } & \multicolumn{5}{|c|}{ Note: Job Family Only After 1997} \\
\hline Job Family & Number: & ४ै। & ৪৷ ২ & ४ि। & ४ै. & अै४े? & अ४। & अి \\
\hline Job Category & & 1996 & 1997 & 1998 & 1999 & 2000 & 2001 & 2002 \\
\hline Health Physics & & & & & & & & \\
\hline Industrial Hygiene & & & & & & . & & \\
\hline Lawyers & & & & & & & & \\
\hline $\begin{array}{l}\text { Personnel/labor } \\
\text { Relations }\end{array}$ & . & & & & & & & \\
\hline Physicians & & & & & & & & \\
\hline $\begin{array}{l}\text { Physician } \\
\text { Assistant/Nurse }\end{array}$ & & & & & & & & \\
\hline $\begin{array}{l}\text { Safeguard \& } \\
\text { Security }\end{array}$ & & & & & & & & \\
\hline $\begin{array}{l}\text { Tech Writers \& } \\
\text { Editors }\end{array}$ & & & & & & & & \\
\hline Trainers & & & & & & & & \\
\hline other & & & & & & & & \\
\hline Subtotal & & & & & & & & \\
\hline $\begin{array}{l}\text { GENERAL } \\
\text { ADMIN/SECRETARYLLLERK }\end{array}$ & ४ै।?: & ?ै: & ?े? & \%थे? & $\mathrm{r}_{2}+\%$ & अथ & $\mathrm{H}_{2}{ }_{2}$ & 2 \\
\hline Admin Assistant & & & & & & & & . \\
\hline Office Clerk (Gen) & 2 & 2 & 2 & & & & & \\
\hline $\begin{array}{l}\text { Office Clerk } \\
\text { (Special) }\end{array}$ & & & & & & & & \\
\hline Secretaries & & & & & & & & \\
\hline $\begin{array}{l}\text { Typist/Word } \\
\text { Processor }\end{array}$ & & & & & & & & \\
\hline other & & & & & & & & \\
\hline
\end{tabular}




\begin{tabular}{|c|c|c|c|c|c|c|c|c|}
\hline \multirow{2}{*}{\multicolumn{9}{|c|}{ Planned Staffing (Furl Time Equivalent) }} \\
\hline Job family $९$ ২ & Number & ४४४ : & 18,3 & $1 \% 3$ & ॥े। & $18+4$ & ৷২४ & \\
\hline Job Category & & 1996 & 1997 & 1998 & 1999 & 2000 & 2001 & 2002 \\
\hline \multicolumn{9}{|l|}{ Subtotal } \\
\hline TECHNICIANS & अै। & २े? & ४ै। & अै। & मे & अ \% & ४९। & \% \\
\hline \multicolumn{9}{|l|}{$\begin{array}{l}\text { Computer } \\
\text { Operator/Coder } \\
\end{array}$} \\
\hline \multicolumn{9}{|l|}{ Drafters } \\
\hline \multicolumn{9}{|l|}{ Engineers/Technicians } \\
\hline \multicolumn{9}{|l|}{$\begin{array}{l}\text { Environmentar } \\
\text { Science } \\
\text { Technicians }\end{array}$} \\
\hline \multicolumn{9}{|l|}{$\begin{array}{l}\text { Health Physics } \\
\text { Technicians }\end{array}$} \\
\hline \multicolumn{9}{|l|}{$\begin{array}{l}\text { Industrial } \\
\text { Saf/health } \\
\text { Technicians }\end{array}$} \\
\hline \multicolumn{9}{|l|}{$\begin{array}{l}\text { Instru/Control } \\
\text { Technicians }\end{array}$} \\
\hline \multicolumn{9}{|l|}{ Lab. Technicians } \\
\hline \multicolumn{9}{|l|}{ Media Technicians } \\
\hline \multicolumn{9}{|l|}{$\begin{array}{l}\text { Survey/Map } \\
\text { Technicians }\end{array}$} \\
\hline \multicolumn{9}{|l|}{ other } \\
\hline \multicolumn{9}{|l|}{ Subtotal } \\
\hline ERAFTS $\%$ & १५ $14 \%$ & \% $125 \%$ & $2 \% \%$ & $2 \%$ & अै। & अै?े & ২९ै। & Pै: \\
\hline Carpenters & 8 & 6 & & & & & & \\
\hline
\end{tabular}




\begin{tabular}{|c|c|c|c|c|c|c|c|c|}
\hline \multicolumn{4}{|c|}{ Planned Staffing (Full Time Equivalent) } & \multicolumn{5}{|c|}{ Note: Job Family Only After 1997} \\
\hline Job Family y 3 ? & Number & s? & ? & +ि & ४ै४४९ & अथे & +४। & अ। \\
\hline Job Category & & 1996 & 1997 & 1998 & 1999 & 2000 & 2001 & 2002 \\
\hline Electricians. & 28 & 28 & & & & & & \\
\hline HVAC & & & & & & & & \\
\hline Machinists & & & & & & & & \\
\hline Masons & & & & & & & & \\
\hline Millwrights & 1 & 1 & & & & & & \\
\hline Painters & 1 & 1 & & & & & & \\
\hline Plmbrs/Pipefitters & 40 & 40 & & & & & & \\
\hline $\begin{array}{l}\text { Struct/Metal } \\
\text { Workers }\end{array}$ & & & . & & & & & \\
\hline $\begin{array}{l}\text { Vehicl/Mobile } \\
\text { Equip. } \\
\text { Mechanics }\end{array}$ & & & & & & & & \\
\hline Welders & & & & & & & & \\
\hline Other & 68 & 49 & & & & & & \\
\hline Subtotal & & & & & & & & \\
\hline OPERATORS & $2 \% 8$ & $8 \%$ & अै। & अै? & ४ै। & अै। & अै। & अ. \\
\hline Lt. Vehicle Drivers & 8 & 8 & & & & & & \\
\hline $\begin{array}{l}\text { Material Moving } \\
\text { Equip. }\end{array}$ & & & & & & & & \\
\hline Nuclear Plant & & & & & & & & \\
\hline $\begin{array}{l}\text { Utilities Waste } \\
\text { Proces }\end{array}$ & & & & & & & & \\
\hline Other & & & & & & & & \\
\hline Subtotal & & & & & & & & \\
\hline
\end{tabular}




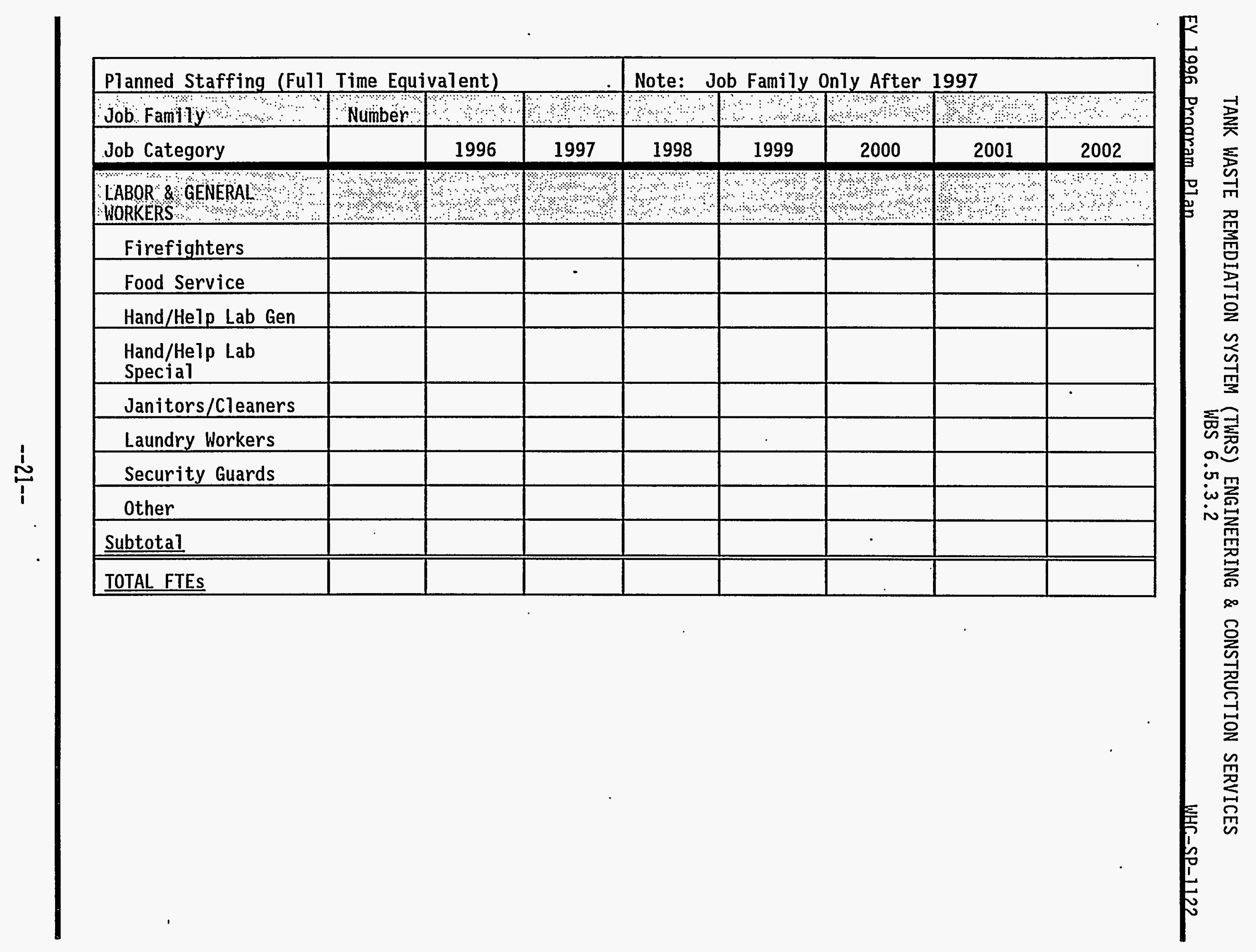




\subsubsection{Spent Nuclear Fuels (SNF) (WBS 6.5.3.3)}

\section{Mission}

The Spent Nuclear Fuels and Path Forward Projects Division, in its capacity as the functional project management entity, has the responsibility for the supervision and support of Construction Forces and ES\&H activities associated with the Division.

\section{Product/Services Provided}

The Spent Nuclear Fuels Division provides the following: Liaison between ICF $\mathrm{KH}$ and WHC projects personnel and plant operators for CF activities; technical expertise to field problems; input to corrective actions resulting from accidents, audit findings, or surveillance; safety walkdowns and inspections; Kaiser Injury Prevention System (KIPS) data base; procedures to implement requirements of WHC-CM-7-5 consistently throughout ICF KH; input to the ICF KH portion of the Hanford Annual Dangerous Waste Report.

\section{Primary Customers}

The primary customers of the Spent Nuclear Fuels Division are: ICF Kaiser Hanford, Westinghouse Hanford Company, and U.S. Department of Energy (RL). 


\begin{tabular}{|c|c|c|c|c|c|c|c|c|}
\hline \multicolumn{4}{|c|}{ Planned Staffing (Full Time Equivalent) } & \multicolumn{5}{|c|}{ Note: Job Family Only After 1997} \\
\hline Job Family & Number: & ২৮? & ४४े\% & \% & अ४ & ४४४४ & ४४४ & १४४ \\
\hline Job Category & & 1996 & 1997 & 1998 & 1999 & 2000 & 2001 & 2002 \\
\hline MANAGERS & १ & ४ै। & ४ै?: & ४ै? & ४ै। & अै० & \%॰ & ४ै० \\
\hline \multicolumn{9}{|l|}{ First line } \\
\hline \multicolumn{9}{|l|}{ General/executive } \\
\hline \multicolumn{9}{|l|}{ Projec/Program } \\
\hline \multicolumn{9}{|l|}{ Other } \\
\hline \multicolumn{9}{|l|}{ Subtotal } \\
\hline ENGINEERS $\% \%$ & ?०.\%? & अे & १ै। & $\% 3 \%$ & 33 & १ै 3 ? & ?. 3 \% & के $3 \ldots$ \\
\hline \multicolumn{9}{|l|}{ Chemical } \\
\hline \multicolumn{9}{|l|}{ Civil } \\
\hline \multicolumn{9}{|l|}{ Computer } \\
\hline \multicolumn{9}{|l|}{ Electrical } \\
\hline \multicolumn{9}{|l|}{ Environmental } \\
\hline \multicolumn{9}{|l|}{ Industrial } \\
\hline \multicolumn{9}{|l|}{ Mechanical } \\
\hline \multicolumn{9}{|l|}{ Nuclear } \\
\hline \multicolumn{9}{|l|}{ Petroleum/Mining } \\
\hline \multicolumn{9}{|l|}{ Plant } \\
\hline \multicolumn{9}{|l|}{ Quality Control } \\
\hline Safety & 3 & 3 & 3. & & & & & \\
\hline \multicolumn{9}{|l|}{ Other } \\
\hline Subtotal & & & & & & & & \\
\hline
\end{tabular}




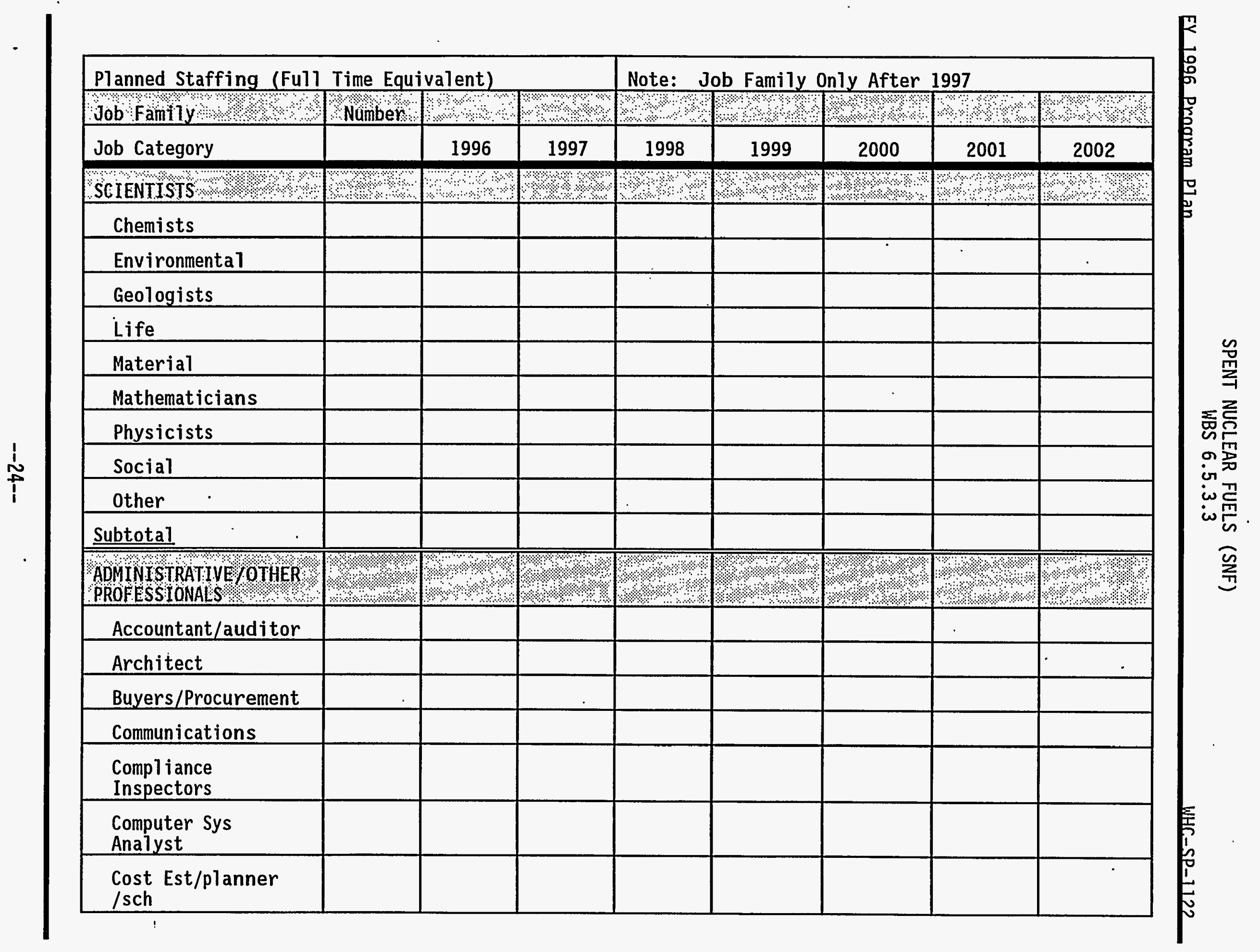




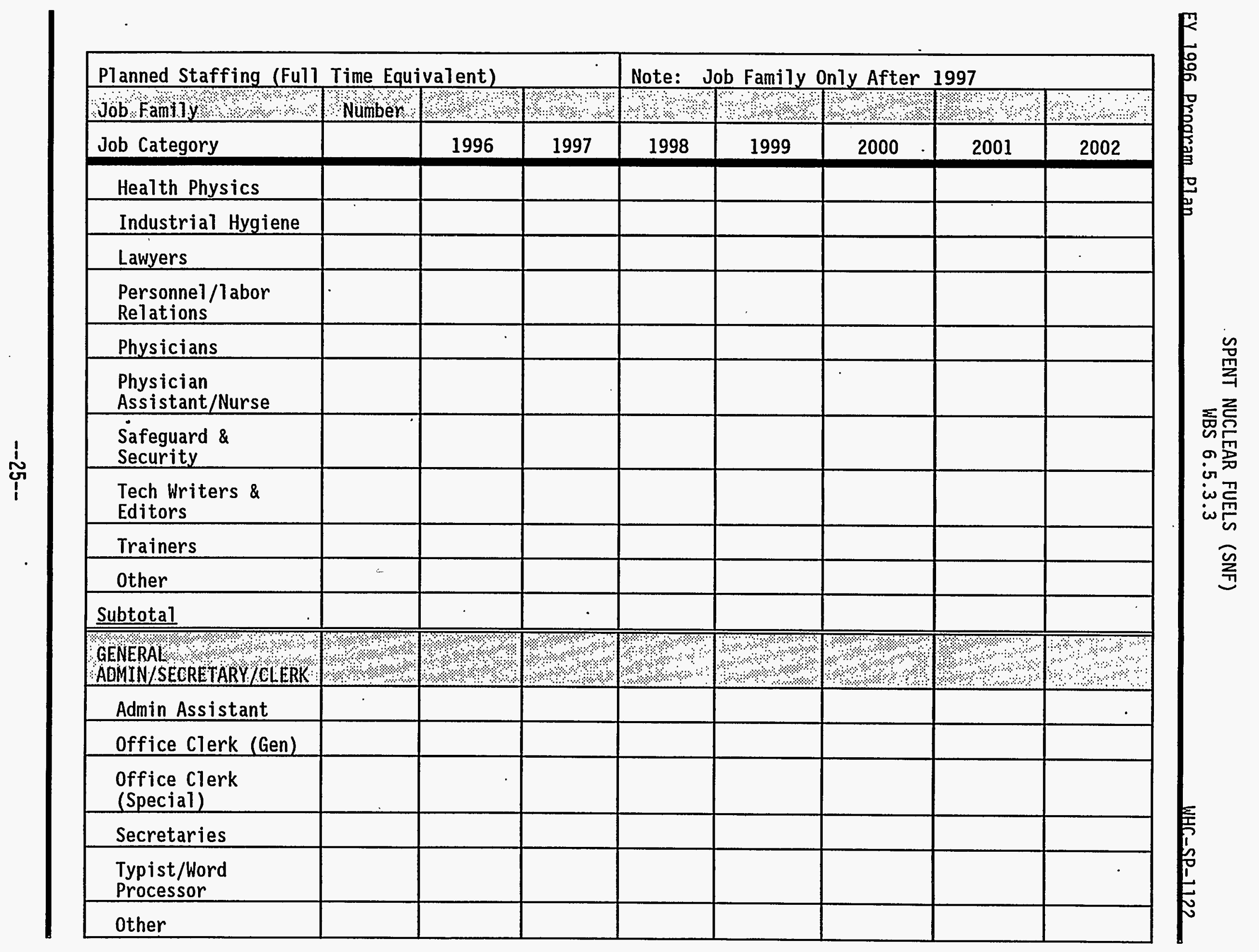




\begin{tabular}{|c|c|c|c|c|c|c|c|c|}
\hline \multicolumn{4}{|c|}{ Planned Staffing (Full Time Equivalent) } & \multicolumn{5}{|c|}{ Note: Job Family Only After 1997} \\
\hline Job Family & Number: & अे? & ४०० & ४ै? & \%॰ & ४४? & ४२२२ & अ४४ \\
\hline Job Category & & 1996 & 1997 & 1998 & 1999 & 2000 & 2001 & 2002 \\
\hline \multicolumn{9}{|l|}{ Subtotal } \\
\hline JECHNICIANS & ४ै०े & अे \%? & ए? ? & ४ै। & ४०४\%? & ४४४४४ & ४ै। & अै। \\
\hline \multicolumn{9}{|l|}{$\begin{array}{l}\text { Computer } \\
\text { Operator/Coder }\end{array}$} \\
\hline \multicolumn{9}{|l|}{ Drafters } \\
\hline \multicolumn{9}{|l|}{ Engineers/Technicians } \\
\hline \multicolumn{9}{|l|}{$\begin{array}{l}\text { Environmental } \\
\text { Science } \\
\text { Technicians }\end{array}$} \\
\hline \multicolumn{9}{|l|}{$\begin{array}{l}\text { Health Physics } \\
\text { Technicians }\end{array}$} \\
\hline \multicolumn{9}{|l|}{$\begin{array}{l}\text { Industrial } \\
\text { Saf/health } \\
\text { Technicians }\end{array}$} \\
\hline \multicolumn{9}{|l|}{$\begin{array}{l}\text { Instru/Control } \\
\text { Technicians }\end{array}$} \\
\hline \multicolumn{9}{|l|}{ Lab. Technicians } \\
\hline \multicolumn{9}{|l|}{ Media Technicians } \\
\hline \multicolumn{9}{|l|}{$\begin{array}{l}\text { Survey/Map } \\
\text { Technicians }\end{array}$} \\
\hline \multicolumn{9}{|l|}{ Other } \\
\hline \multicolumn{9}{|l|}{ Subtotal } \\
\hline CRAFTS $\%$ ২ & $\%, 24 \%$ & $36 \%$ & \% \% \% & अे०े. & ४ै। & अ ४ै। & १४०० & a \\
\hline Carpenters & 2 & 3 & & & & & & \\
\hline
\end{tabular}




\begin{tabular}{|c|c|c|c|c|c|c|c|c|}
\hline \multicolumn{4}{|c|}{ Planned Staffing (Ful1 Time Equivalent) } & \multicolumn{5}{|c|}{ Note: Job Family Only After 1997} \\
\hline Job Famky & Number & ४ै०. & ४४ & ॐ & ४४? & ॠथ०2 & ए. & 14\% \\
\hline Job Category & & 1996 & 1997 & 1998 & 1999 & 2000 & 2001 & 2002 \\
\hline Electricians & 2 & 6 & & & & & & \\
\hline \multicolumn{9}{|l|}{ HVAC } \\
\hline \multicolumn{9}{|l|}{ Machinists } \\
\hline \multicolumn{9}{|l|}{ Masons } \\
\hline \multicolumn{9}{|l|}{ Millwrights } \\
\hline Painters & & 4 & & & & & & \\
\hline Plmbrs/Pipefitters & 4 & 2 & & & & & & \\
\hline $\begin{array}{l}\text { Struct/Metal } \\
\text { Workers }\end{array}$ & 5 & 4 . & & & & & & \\
\hline \multicolumn{9}{|l|}{$\begin{array}{l}\text { Vehicl/Mobile } \\
\text { Equip. } \\
\text { Mechanics }\end{array}$} \\
\hline \multicolumn{9}{|l|}{ Welders } \\
\hline other & 11 & 17 & & & & & & \\
\hline \multicolumn{9}{|l|}{ Subtotal } \\
\hline OPERATORS & 5,0 & \% 4 & \%? & ए? & ॠथ & 20 & एक. & $\%$ \\
\hline Lt. Vehicle Drivers & 5 & 4 & & & & & & \\
\hline \multicolumn{9}{|l|}{$\begin{array}{l}\text { - Material Moving } \\
\text { Equip. }\end{array}$} \\
\hline \multicolumn{9}{|l|}{ Nuclear Plant } \\
\hline \multicolumn{9}{|l|}{$\begin{array}{l}\text { Utilities Waste } \\
\text { Proces }\end{array}$} \\
\hline \multicolumn{9}{|l|}{ Other } \\
\hline Subtotal & & & & & & & & \\
\hline
\end{tabular}




\begin{tabular}{|c|c|c|c|c|c|c|c|c|}
\hline \multicolumn{4}{|c|}{ Planned Staffing (Full Time Equivalent) } & \multicolumn{5}{|c|}{ Note: Job Family Only After 1997} \\
\hline Job Family & Number: & अे। & ॠै & ২২? & खे। & ४४४। & ४४৷ & এ४४ \\
\hline Job Category & & 1996 & 1997 & 1998 & 1999 & 2000 & 2001 & 2002 \\
\hline $\begin{array}{l}\text { GABOR \& GENERAL } \\
\text { WORKERS }\end{array}$ & ४ै? & अ४े? & अै? & $\%$ & 4 & अ? & \%ै? & अै? \\
\hline Firefighters & & & & & & & & \\
\hline Food Service & & & & & & & & \\
\hline Hand/Help Lab Gen & & & & & & & & \\
\hline $\begin{array}{l}\text { Hand/Help Lab } \\
\text { Special }\end{array}$ & & & & & & & & \\
\hline Janitors/Cleaners & & & & & & & & \\
\hline Laundry Workers & & & & & & & & \\
\hline Security Guards & & & & & & & & \\
\hline other & & & & & & & . & \\
\hline Subtotal & & & & & & & & \\
\hline TOTAL FTES & & & & & & & & \\
\hline
\end{tabular}


3.1.4 PNL Landlord, Engineering and Construction Services (WBS 6.5.3.4)

Mission

The PNL Landlord, Engineering and Construction Services Division, in it capacity as the functional project management entity, has the responsibility for the supervision and support of Construction Forces and ES\&H activities associated with the Division.

\section{Product/Services Provided}

The PNL Landlord, Engineering and Construction Forces Division provides the following: Liaison between ICF KH and WHC projects personnel and plant operators for CF activities; technical expertise to field problems; input to corrective actions resulting from accidents, audit findings, or surveillance; safety walkdowns and inspections; Kaiser Injury Prevention System (KIPS) data base; procedures to implement requirements of WHC-CM-7-5 consistently throughout ICF KH; input to the ICF KH portion of the Hanford Annual Dangerous Waste Report.

\section{Primary Customer}

The primary customers of the PNL Landlord, Engineering and Construction Services are: ICF Kaiser Hanford, Westinghouse Hanford Company, Pacific Northwest Laboratory, and U.S. Department of Energy. 


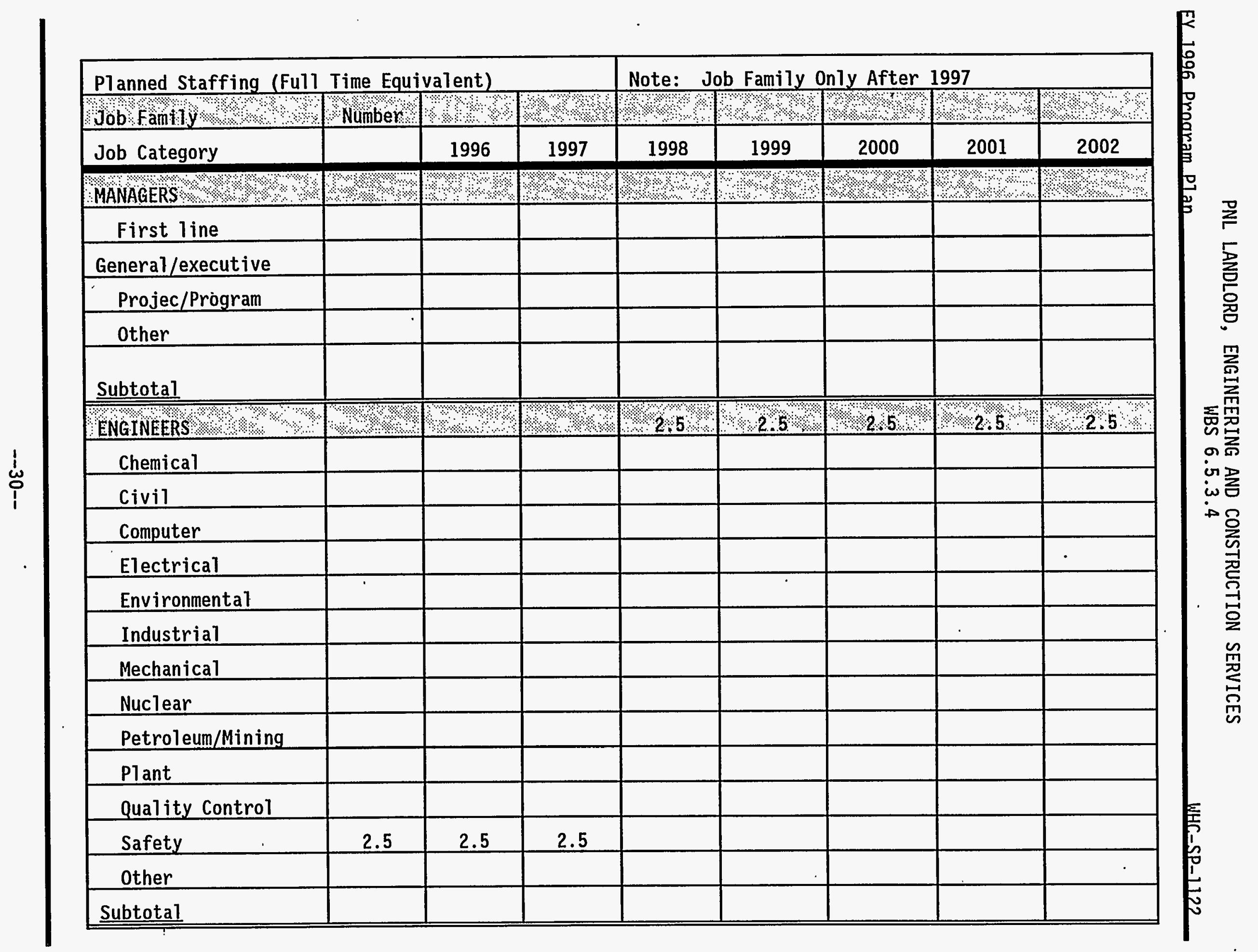




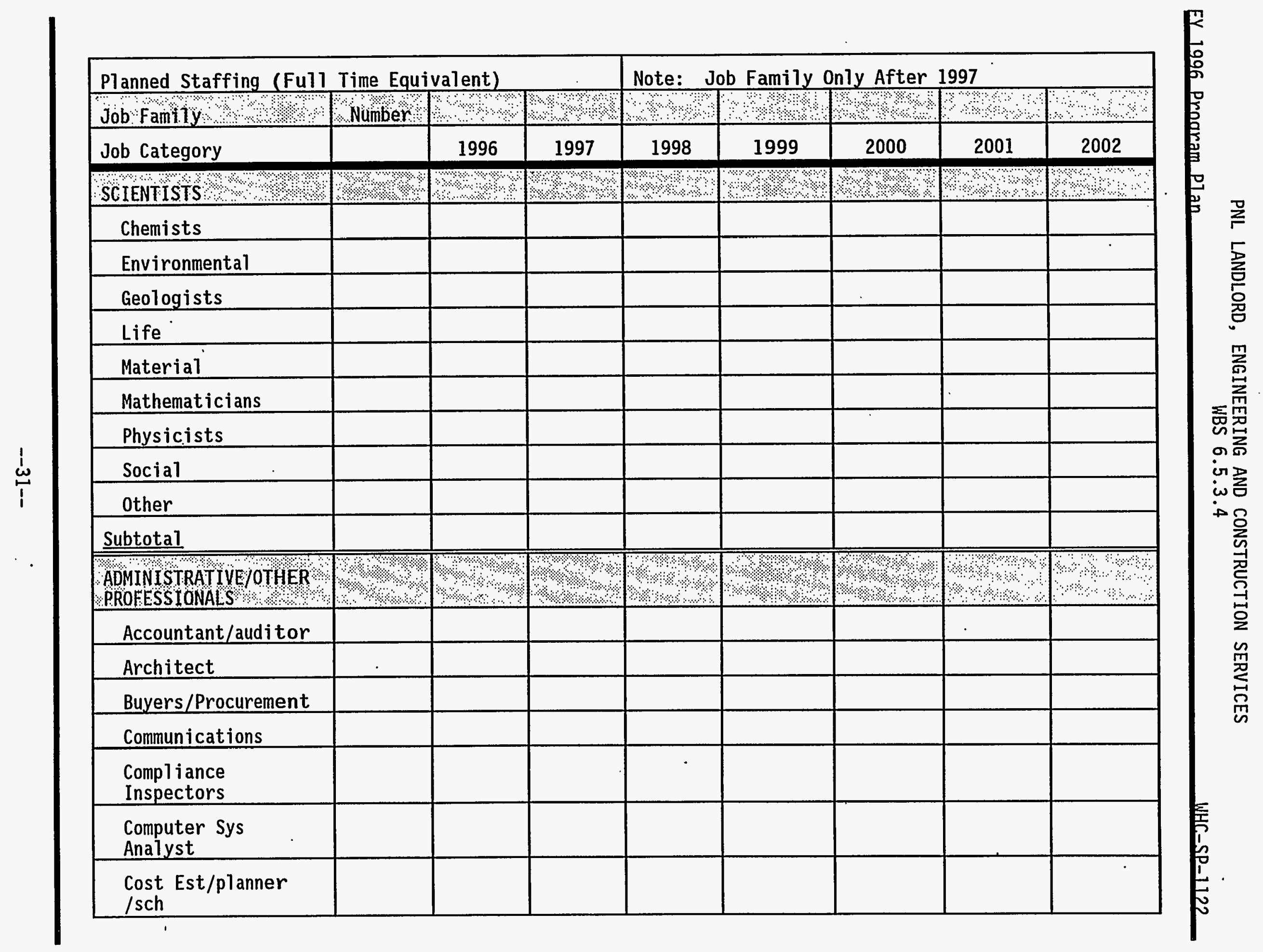




\begin{tabular}{|c|c|c|c|c|c|c|c|c|}
\hline \multicolumn{4}{|c|}{ Planned Staffing (Full Time Equivalent) } & \multicolumn{5}{|c|}{ Note: Job Family Only After 1997} \\
\hline Job Famnily & Number: & יथ०० & 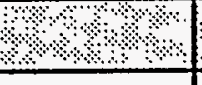 & ॰ & अ४००\% & ४४४४? & ? & अ४४\% \\
\hline Job Category & & 1996 & 1997 & 1998 & 1999 & 2000 & 2001 & 2002 \\
\hline \multicolumn{9}{|l|}{ Health Physics } \\
\hline \multicolumn{9}{|l|}{ Industrial Hygiene } \\
\hline \multicolumn{9}{|l|}{ Lawyers } \\
\hline \multicolumn{9}{|l|}{$\begin{array}{l}\text { Personnel/labor } \\
\text { Relations }\end{array}$} \\
\hline \multicolumn{9}{|l|}{ Physicians } \\
\hline \multicolumn{9}{|l|}{$\begin{array}{l}\text { Physician } \\
\text { Assistant/Nurse }\end{array}$} \\
\hline \multicolumn{9}{|l|}{$\begin{array}{l}\text { Safeguard \& } \\
\text { Security }\end{array}$} \\
\hline \multicolumn{9}{|l|}{$\begin{array}{l}\text { Tech Writers \& } \\
\text { Editors }\end{array}$} \\
\hline \multicolumn{9}{|l|}{ Trainers } \\
\hline \multicolumn{9}{|l|}{ other } \\
\hline \multicolumn{9}{|l|}{ Subtotal } \\
\hline $\begin{array}{l}\text { GeNERAL } \\
\text { ADMIN/SECRETARY/CLERK }\end{array}$ & 2 & 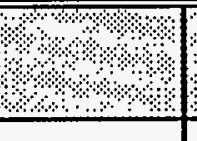 & $2+2$ & r. & भै। & ए४० & $?^{\circ} \div$ & के० \\
\hline \multicolumn{9}{|l|}{ Admin Assistant } \\
\hline \multicolumn{9}{|l|}{ Office Clerk (Gen) } \\
\hline \multicolumn{9}{|l|}{$\begin{array}{l}\text { Office Clerk } \\
\text { (Special) }\end{array}$} \\
\hline \multicolumn{9}{|l|}{ Secretaries } \\
\hline \multicolumn{9}{|l|}{$\begin{array}{l}\text { Typist/Word } \\
\text { Processor }\end{array}$} \\
\hline other & & & & & & & & \\
\hline
\end{tabular}




\begin{tabular}{|c|c|c|c|c|c|c|c|c|}
\hline \multicolumn{4}{|c|}{ Planned Staffing (Full Time Equivalent) } & \multicolumn{5}{|c|}{ Note: Job Family Only After 1997} \\
\hline Job Fami $y$ अ & Nümber & ४४ & अ४। & ४४ & +४ & अै४? & अ & से \\
\hline Job Category & & 1996 & 1997 & 1998 & 1999 & 2000 & 2001 & 2002 \\
\hline Subtotal & & & & & & & & \\
\hline TECHNICIANS & ४ै४े। & के। & ४। & +. & ४। ४ै। & अे? & अ४। & अ \\
\hline $\begin{array}{l}\text { Computer } \\
\text { Operator/Coder }\end{array}$ & & & & & & & & \\
\hline Drafters & & & & & & & & \\
\hline Engineers/Technicians & & & & & & & & \\
\hline $\begin{array}{l}\text { Environmental } \\
\text { Science } \\
\text { Technicians } \\
\end{array}$ & & & & & & & & \\
\hline $\begin{array}{l}\text { Health Physics } \\
\text { Technicians } \\
\end{array}$ & & & & . & & & & \\
\hline $\begin{array}{l}\text { Industrial } \\
\text { Saf/health } \\
\text { Technicians } \\
\end{array}$ & & & & & & & & \\
\hline $\begin{array}{l}\text { Instru/Control } \\
\text { Technicians }\end{array}$ & & & & & & & & \\
\hline Lab. Technicians & & & & & & & & \\
\hline Media Technicians & & & & & & & & \\
\hline $\begin{array}{l}\text { Survey/Map } \\
\text { Technicians }\end{array}$ & & & & & & & & \\
\hline Other & & & & & . & & & \\
\hline Subtotal & & & & & & & & \\
\hline CRATTS $\% ८ ४ \%$ & 18108. & \%. $99 \%$ & & \%०। & ২े? & \%०४४। & १४०े & के \\
\hline Carpenters & 14 & 13 & & & & & & \\
\hline
\end{tabular}




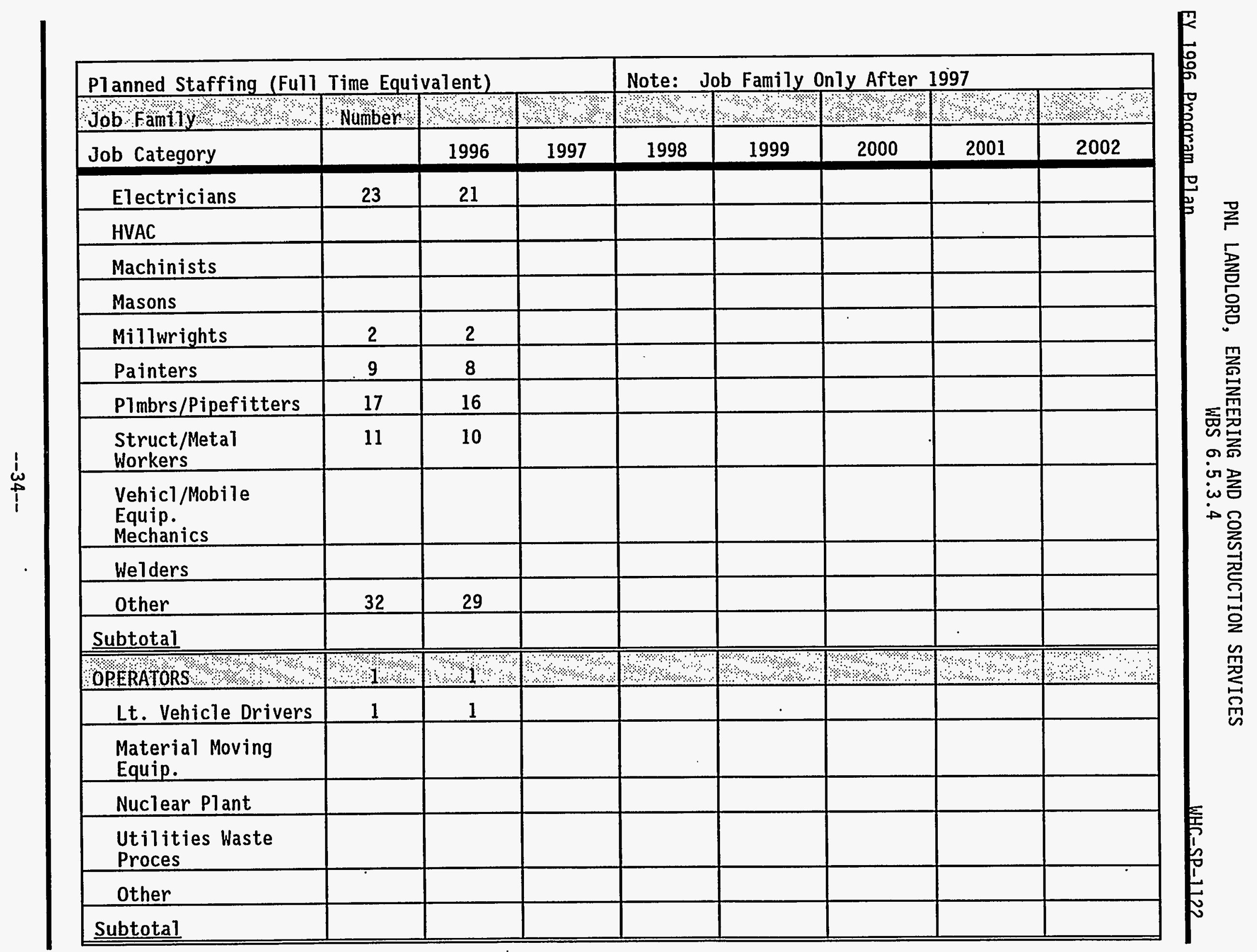




\begin{tabular}{|c|c|c|c|c|c|c|c|c|}
\hline \multicolumn{4}{|c|}{ Planned Staffing (Full Time Equivalent) } & \multicolumn{5}{|c|}{ Note: Job Family Only After 1997} \\
\hline Job Fam11y & Nümber. & 3 & \%० & +: & \%। & ४४ & 3 & 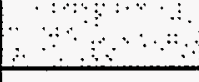 \\
\hline Job Category & & 1996 & 1997 & 1998 & 1999 & 2000 & 2001 & 2002 \\
\hline $\begin{array}{l}\text { LABOR } 8 \text { GENERAL } \\
\text { WORKERS }\end{array}$ & ?४४ & \%ै। & ४ै। & अे & अ४। & $4 \%$ & $14 \%$ & (4) \\
\hline \multicolumn{9}{|l|}{ Firefighters } \\
\hline \multicolumn{9}{|l|}{ Food Service } \\
\hline \multicolumn{9}{|l|}{ Hand/Help Lab Gen } \\
\hline \multicolumn{9}{|l|}{$\begin{array}{l}\text { Hand/Help Lab } \\
\text { Special }\end{array}$} \\
\hline \multicolumn{9}{|l|}{ Janitors/Cleaners } \\
\hline \multicolumn{9}{|l|}{ Laundry Workers } \\
\hline \multicolumn{9}{|l|}{ Security Guards } \\
\hline \multicolumn{9}{|l|}{ other } \\
\hline \multicolumn{9}{|l|}{ Subtotal } \\
\hline TOTAL FTES & & & & & & & & \\
\hline
\end{tabular}


3.1.5 Transition Projects/Site Engineering \& Construction Services (WBS $6.5 .3 .5)$

Mission

The Transition Project/Site Engineering \& Construction Services Division, in its capacity as the functional construction/management entity, has the responsibility for the supervision and support of the Construction Forces and ES\&H activities associated with the Division.

\section{Product/Services Provided}

The Transition Projects/Site Engineering \& Construction Services Division provides the following: Liaison between ICF KH and WHC projects personnel and plant operators for CF activities in support of the WHC Facility Transition. organization; technical expertise to field problems; input to corrective actions resulting from accidents, audit findings, or surveillance; safety walkdowns and inspections; Kaiser Injury Prevention System (KIPS) data base; procedures to implement requirements of WHC-CM-7-5 consistently throughout ICF KH; input to the ICF KH portion of the Hanford Annual Dangerous Waste Report.

\section{Primary Customers}

The primary customers of the Transition Projects/Site Engineering/Construction Services Division are: ICF Kaiser Hanford, Westinghouse Hanford Company, and the U.S. Department of Energy (RL). 


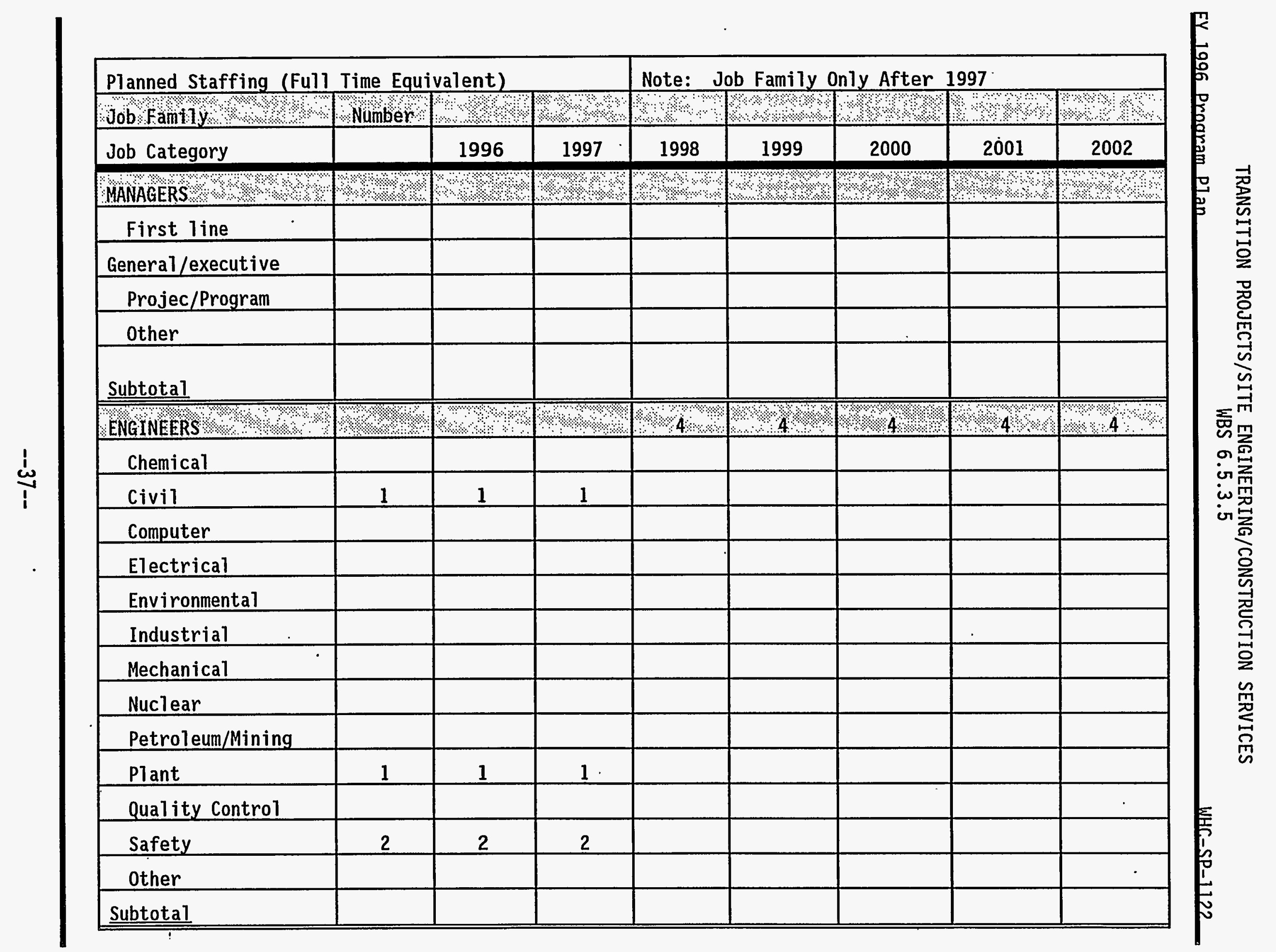




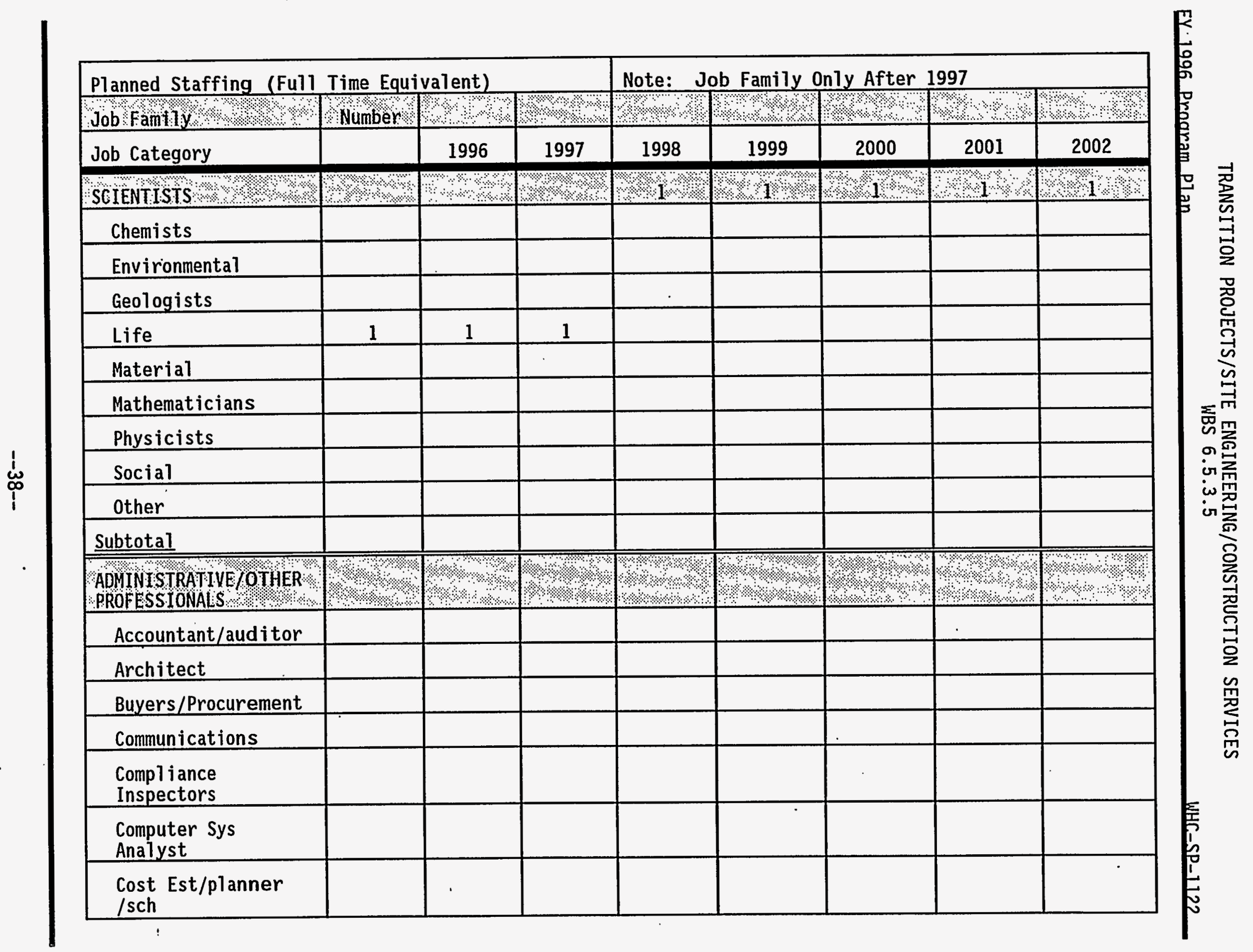




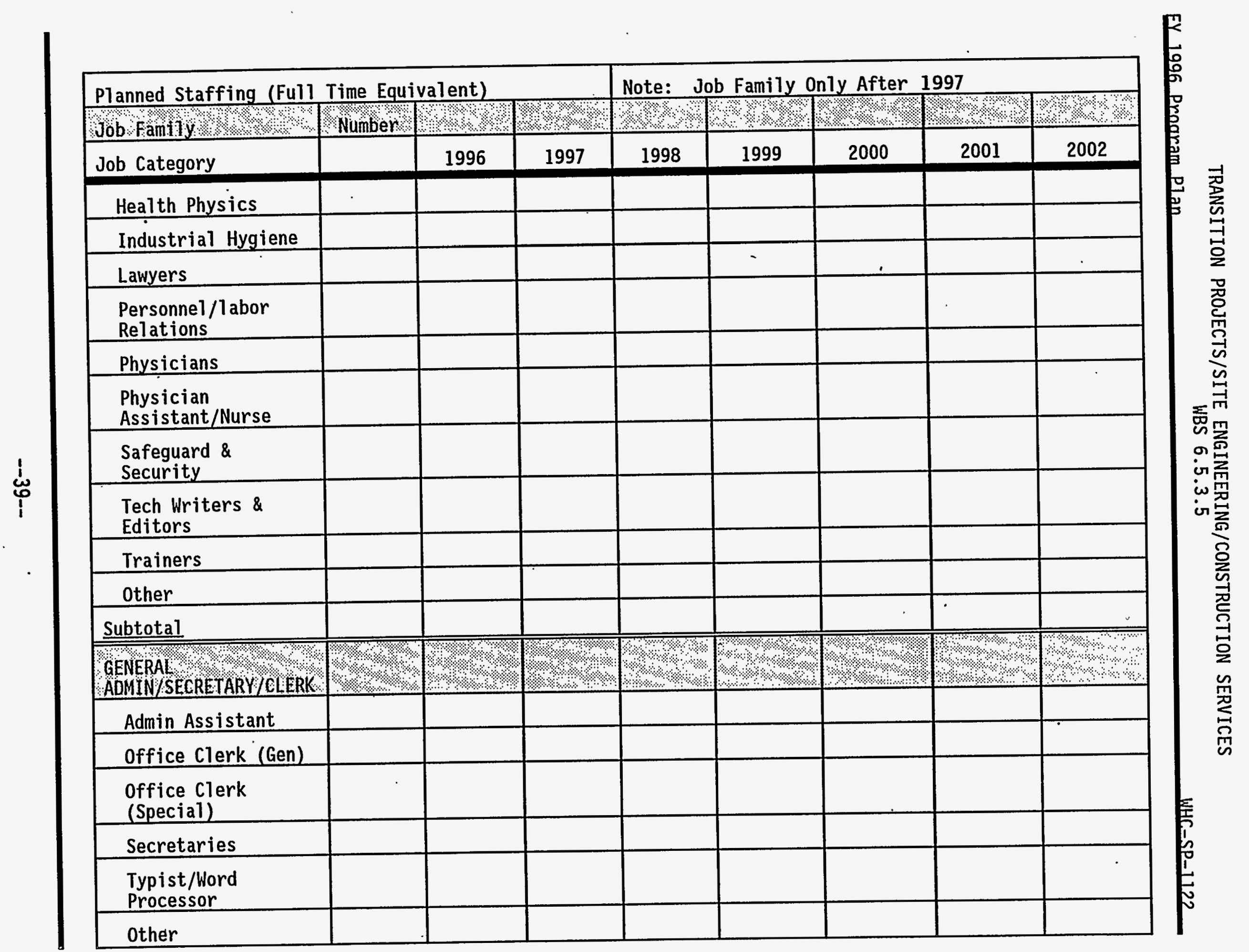




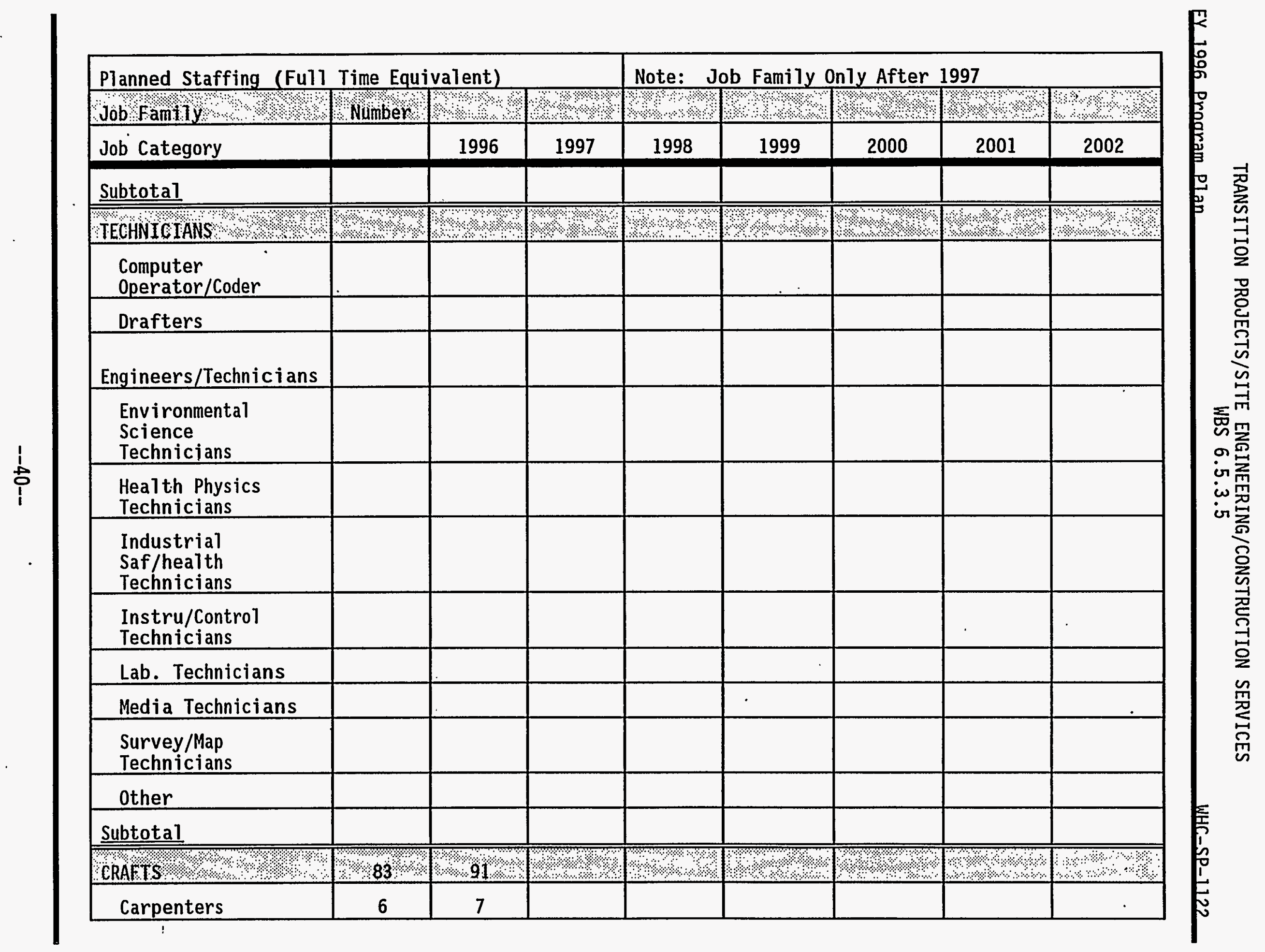




\begin{tabular}{|c|c|c|c|c|c|c|c|c|}
\hline \multicolumn{4}{|c|}{ Planned Staffing (Full Time Equivalent) } & \multicolumn{5}{|c|}{ Note: Job Family Only After 1997} \\
\hline Job Fami1y: ? ? & Number: & अ? & \%ः०० & ४ & ४ै ९) & \% & \%थ & ४৫४४ \\
\hline Job Category & & 1996 & 1997 & 1998 & 1999 & 2000 & 2001 & 2002 \\
\hline Electricians & 20 & 21 & & & & & & \\
\hline \multicolumn{9}{|l|}{ HVAC } \\
\hline \multicolumn{9}{|l|}{ Machinists } \\
\hline Masons & 2 & 2 & & & & & & \\
\hline Millwrights & & & & & & & & . \\
\hline Painters & 7 & 8 . & & & & & & \\
\hline Plmbrs/Pipefitters & 12 & 14 & & & & & & \\
\hline $\begin{array}{l}\text { Struct/Metal } \\
\text { Workers }\end{array}$ & 17 & 19 & & & & & & \\
\hline \multicolumn{9}{|l|}{$\begin{array}{l}\text { Vehicl/Mobile } \\
\text { Equip. } \\
\text { Mechanics }\end{array}$} \\
\hline \multicolumn{9}{|l|}{ Welders } \\
\hline Other & 19 & 20 & & & & & & \\
\hline \multicolumn{9}{|l|}{ Subtotal } \\
\hline OPERATORS & $2 \% 3 \%$ & $\%$ & ২ং. ২০ ৪ & ४०४ & \%े ২. & ४ै ४ै. & 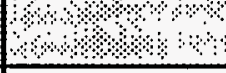 & एल। \\
\hline Lt. Vehicle Drivers & 3 & 4 & & & & & & \\
\hline \multicolumn{9}{|l|}{$\begin{array}{l}\text { Material Moving } \\
\text { Equip. }\end{array}$} \\
\hline \multicolumn{9}{|l|}{ Nuclear Plant } \\
\hline \multicolumn{9}{|l|}{$\begin{array}{l}\text { Utilities Waste } \\
\text { Proces }\end{array}$} \\
\hline \multicolumn{9}{|l|}{ Other } \\
\hline Subtotal & & & & & & & & \\
\hline
\end{tabular}




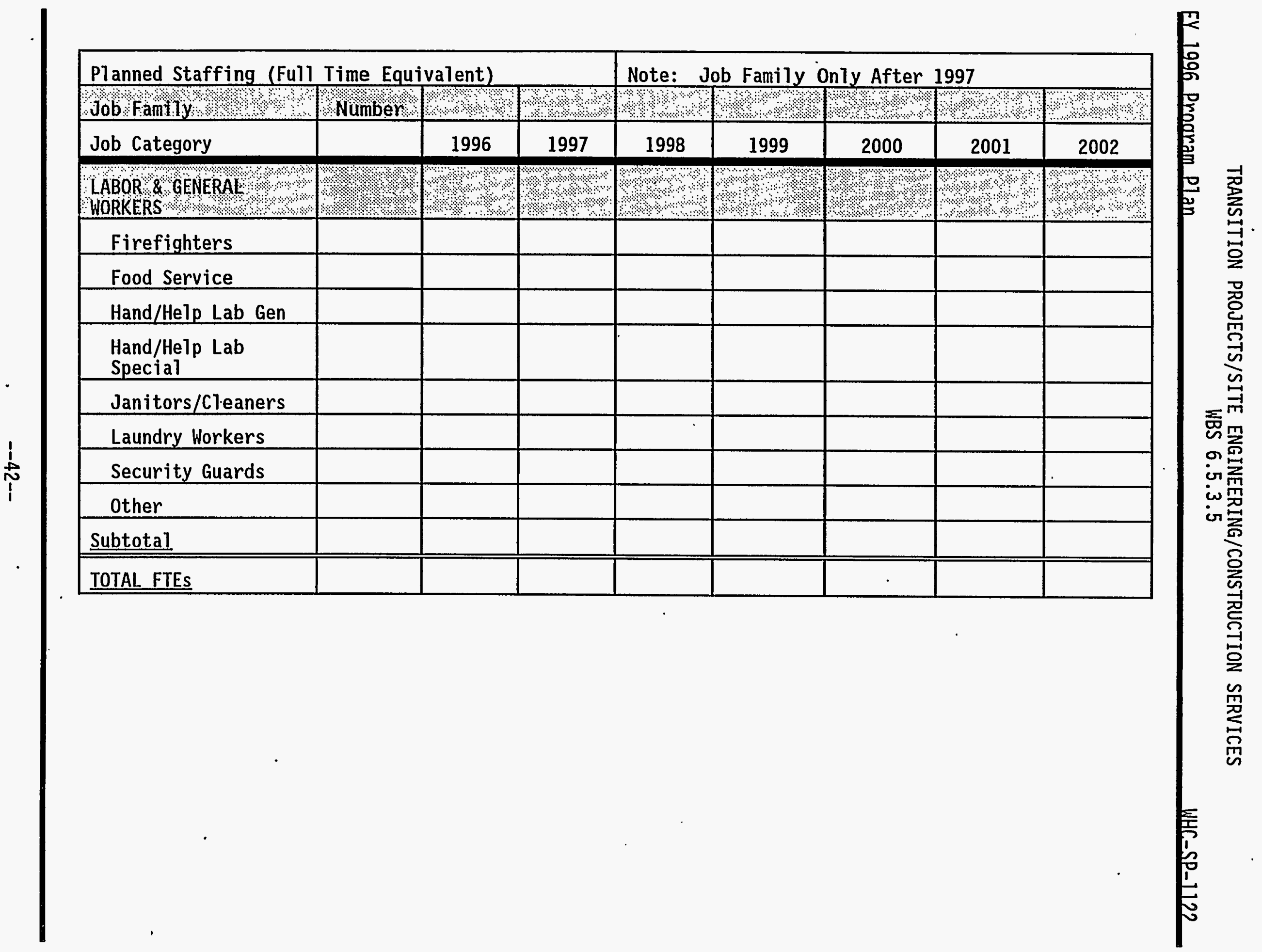




\section{CONSTRUCTION FORCES POOL}

WBS 6.5 .3

FY 1996 Program PTan

WHC-SP-1122

.0 SCHEDULE BASELINE

4.1 Product Deliverables/Milestone List

----NOT APPLICABLE---- 
.0 COST BASELINE SUMMARY

5.1 Cost Baseline

5.2 Planned Staffing Profile 


\section{ICF Kaiser Hanford CF Pool \\ ProposedFY 96 Budget \\ (In Thousands)}

\begin{tabular}{|c|c|c|c|}
\hline & $\begin{array}{c}\text { FY96 } \\
\text { BUDGET }\end{array}$ & $\begin{array}{l}\text { FY } 95 \\
\text { FYSF }\end{array}$ & $\begin{array}{l}\text { ORIGINAL } \\
\text { FY 95 } \\
\text { BUDGET* }\end{array}$ \\
\hline Non-Manual Indirect Labor & 899 & 1,186 & 362 \\
\hline $\begin{array}{l}\text { Craft Direct } \\
\text { Craft Overhead } \\
\text { Total Craft }\end{array}$ & $\begin{array}{r}20,054 \\
1,915 \\
21,969\end{array}$ & $\begin{array}{c}23,424 \\
1,736 \star \\
25,160\end{array}$ & $\begin{array}{r}20,373 \\
2,018 \\
22,391\end{array}$ \\
\hline Total Labor & 22,868 & 26,346 & 22,753 \\
\hline Nonlabor costs & 3,112 & 4,565 & 2,606 \\
\hline Total Budget & 25,980 & 30,911 & 25,359 \\
\hline FTEs & 395.5 & 477 & 440 \\
\hline te & $29.60 \%$ & $31.96 \%$ & $25.20 \%$ \\
\hline
\end{tabular}

* Craft Overheadincludes indirect craft charges to the non-manual pool, which in FY 96 are budgeted in the CF Pool as dictated by the ICF CAS Disclousre Statement.

** The Original Budget for FY 95 was based on an A/EC organizationstructure that is significantly different from the structureafter the $1 / 13 / 95$ re-organization. This resultedin some changes in charging practices. 


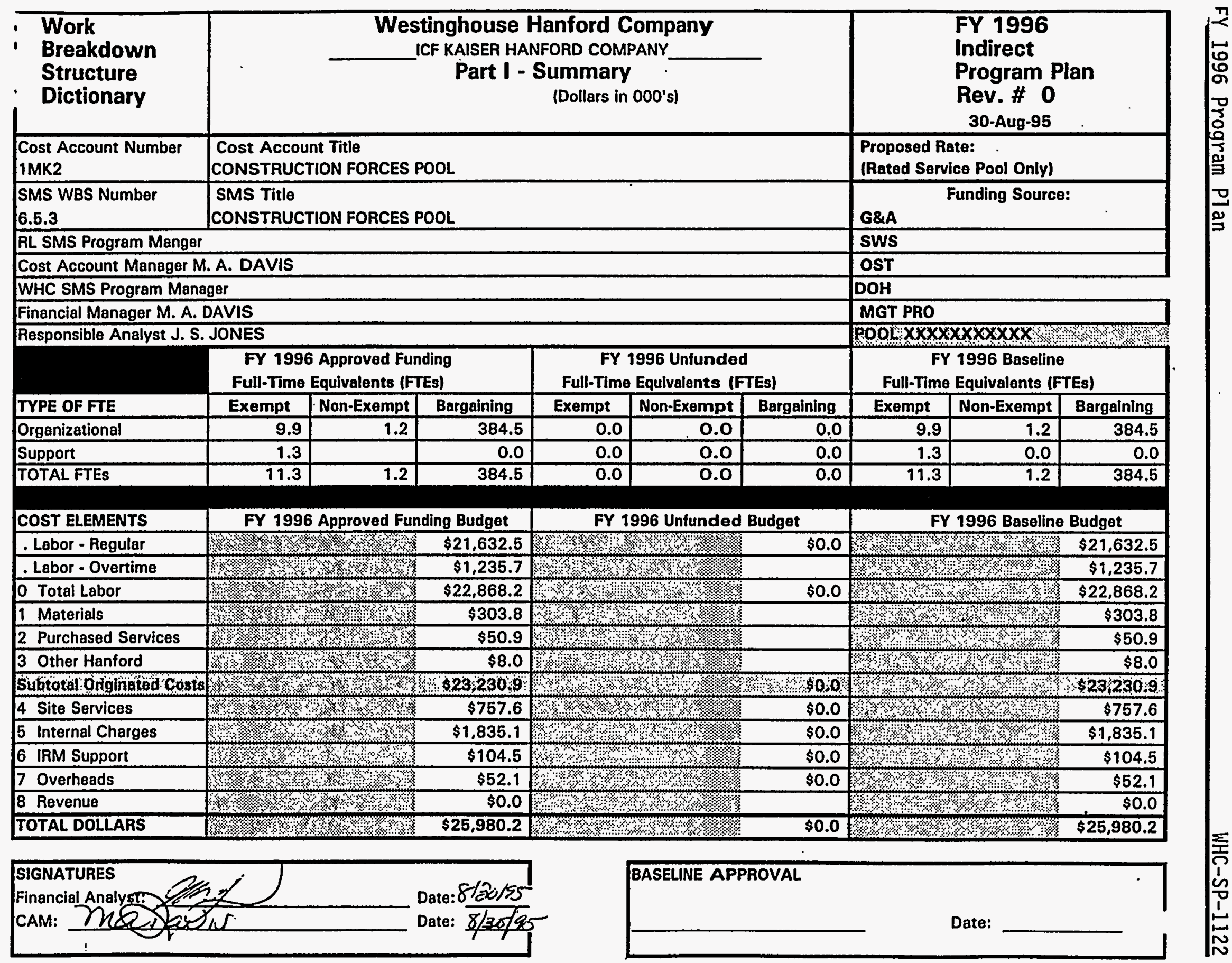




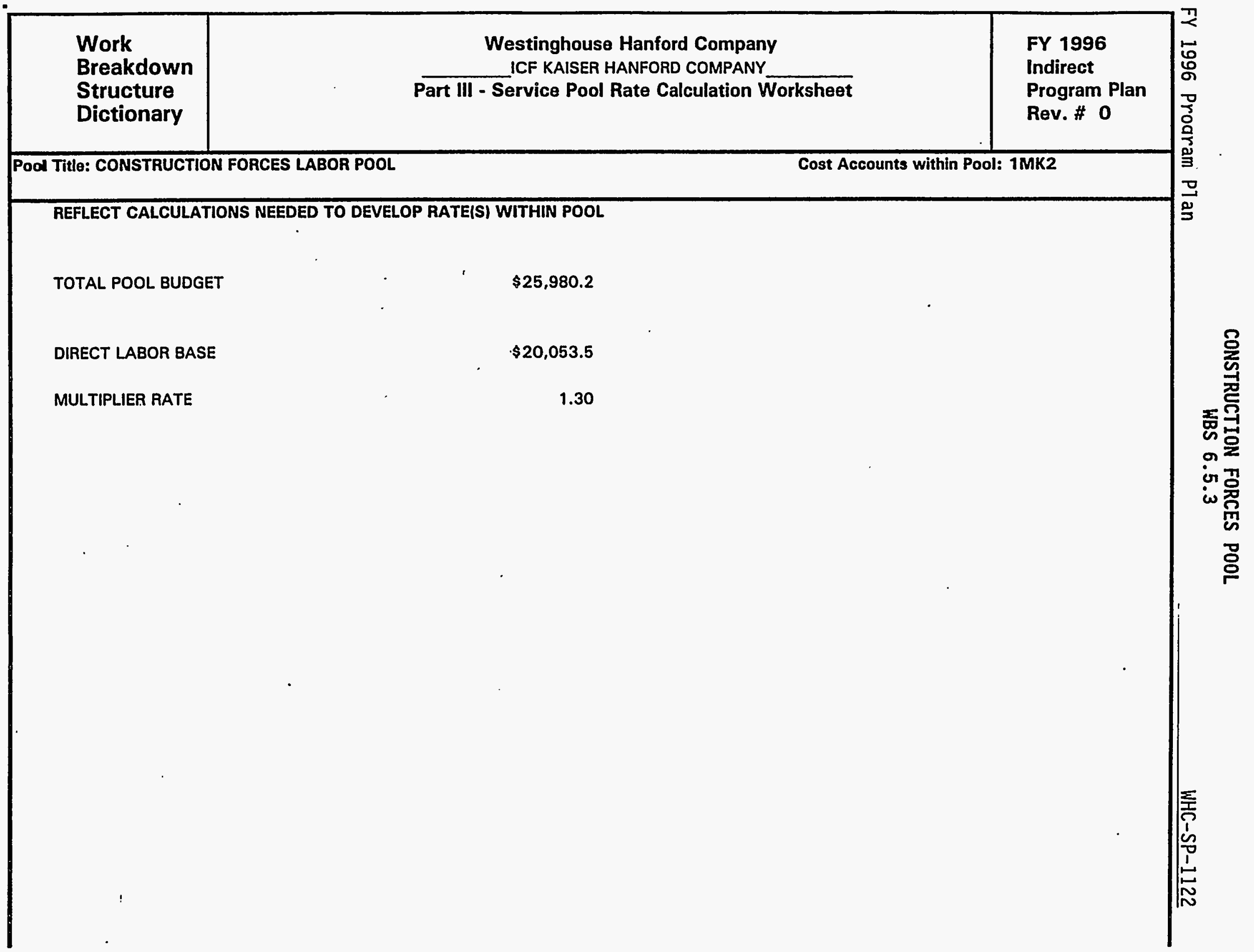




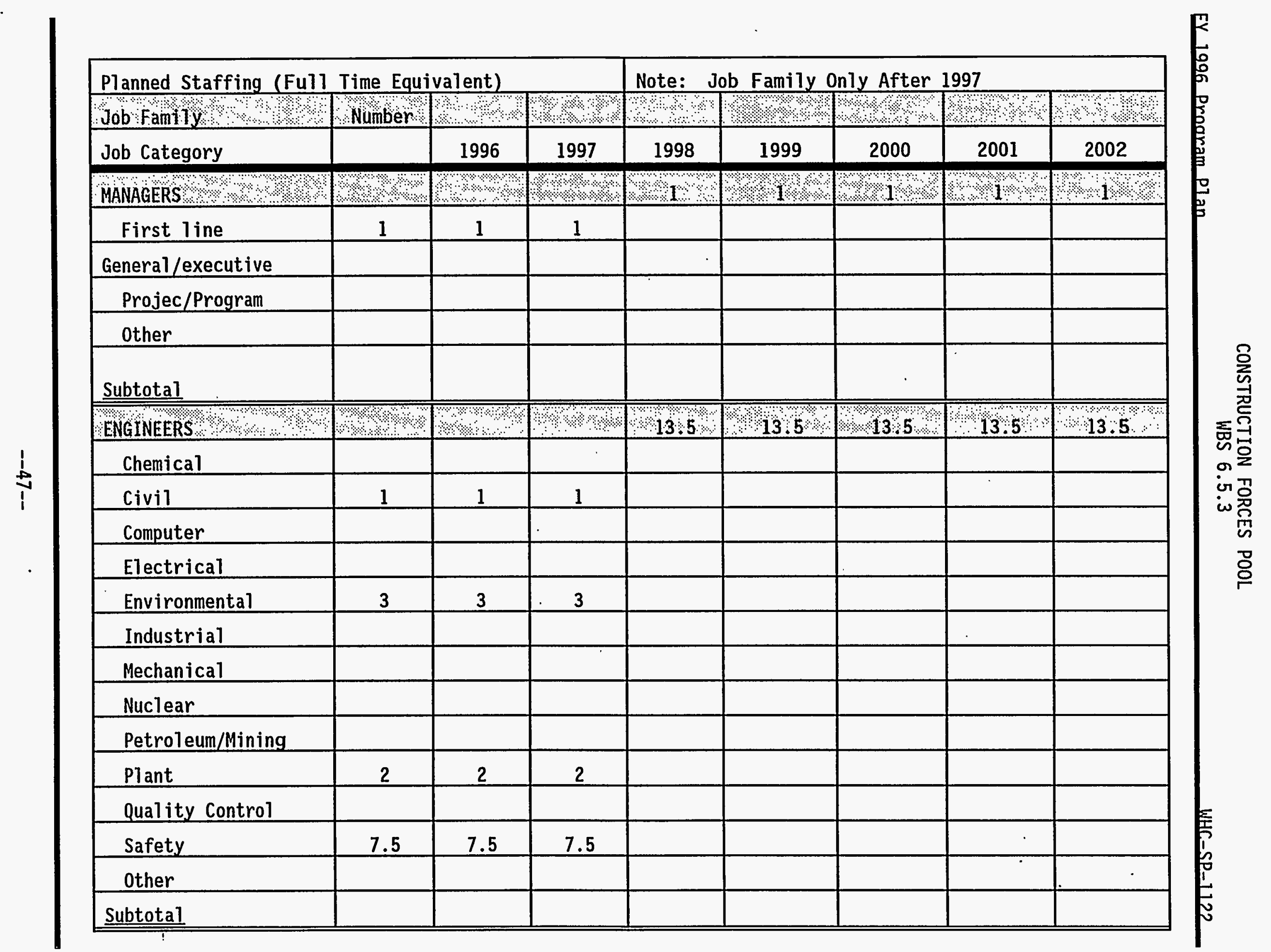




\begin{tabular}{|c|c|c|c|c|c|c|c|c|}
\hline \multicolumn{4}{|c|}{ Planned Staffing (Full Time Equivalent) } & \multicolumn{5}{|c|}{ Note: Job Family Only After 1997} \\
\hline Job Famtly & Number. & १४ & 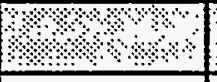 & अे & \%? & ?४े & 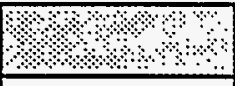 & \%े। \\
\hline Job Category & & 1996 & 1997 & 1998 & 1999 & 2000 & 2001 & 2002 \\
\hline SCIENTISTS & ४४ & ४ै। & $4.8 \div$ & अे। & \%1\% & $8.1 \%$ & $1 \%, 1 \%$ & +1 \\
\hline Chemists & & & & & & & & \\
\hline Environmental & & - & & & & - & & \\
\hline Geologists & & & & & & & & \\
\hline Life & 1 & 1 & 1 & & & & & \\
\hline Material & & & & & & & & \\
\hline Mathematicians & & & & & & & & \\
\hline Physicists & & & & & & & & \\
\hline Social & & & & & & & & \\
\hline Other & - & & & & & & & \\
\hline Subtotal & . & & & & & & & \\
\hline $\begin{array}{l}\text { ADMINISTRATIVE/OTHER } \\
\text { PROFESS IONALS }\end{array}$ & ি২े২े & अै। & १ै०े० & ४ै। बि & मै। & अै। & मे०े? & मिं \\
\hline Accountant/auditor & & & & & & & & \\
\hline Architect & & & & & & & & \\
\hline Buyers/Procurement & & & & & & & & \\
\hline Communications & & & & & & & & \\
\hline $\begin{array}{l}\text { Comp Tiance } \\
\text { Inspectors }\end{array}$ & & & & & & & & \\
\hline $\begin{array}{l}\text { Computer Sys } \\
\text { Analyst }\end{array}$ & & & & & & & & \\
\hline $\begin{array}{l}\text { Cost Est/ planner/ } \\
\text { sch }\end{array}$ & & & & & & & & \\
\hline
\end{tabular}




\begin{tabular}{|c|c|c|c|c|c|c|c|c|}
\hline \multicolumn{4}{|c|}{ Planned Staffing (Full Time Equivalent) } & \multicolumn{5}{|c|}{ Note: Job Family Only After 1997} \\
\hline Job family & Number: & थथम & $2 \%$ & ॠथ & \%०० & 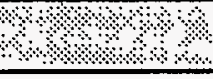 & $2 \times 1 \%$ & $2+2$ \\
\hline Job Category & & 1996 & 1997 & 1998 & 1999 & 2000 & 2001 & 2002 \\
\hline Health Physics & & & & & & & & \\
\hline Industrial Hygiene & & & & & & & & \\
\hline Lawyers & & & & & & & & \\
\hline $\begin{array}{l}\text { Personnel/1 labor } \\
\text { Relations }\end{array}$ & & & & & & & & \\
\hline Physicians & & & & & & & & . \\
\hline $\begin{array}{l}\text { Physician } \\
\text { Assistant/Nurse }\end{array}$ & & & & & & & & \\
\hline $\begin{array}{l}\text { Safeguard \& } \\
\text { Security }\end{array}$ & & & & & & & & \\
\hline $\begin{array}{l}\text { Tech Writers \& } \\
\text { Editors }\end{array}$ & & & & & & & & \\
\hline Trainers & & & & & & & & \\
\hline other & & & & & & & & \\
\hline Subtotal & & & & & & & & \\
\hline $\begin{array}{l}\text { GENERAL } \\
\text { ADMIN/SECRETARY,CLERK }\end{array}$ & 2थ: & 12.8 & 8 & 2.3. & 2 & 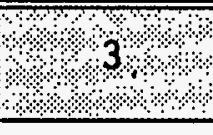 & 3 & 23 \\
\hline Admin Assistant & & & & & & & & \\
\hline Office Clerk (Gen) & 3 & 3 & 3 & & & & & \\
\hline $\begin{array}{l}\text { Office Clerk } \\
\text { (Special) }\end{array}$ & & & & & & & & \\
\hline Secretaries & & & & & & & & \\
\hline $\begin{array}{l}\text { Typist/Word } \\
\text { Processor }\end{array}$ & & & & & & & & \\
\hline other & & & & & & & & \\
\hline
\end{tabular}




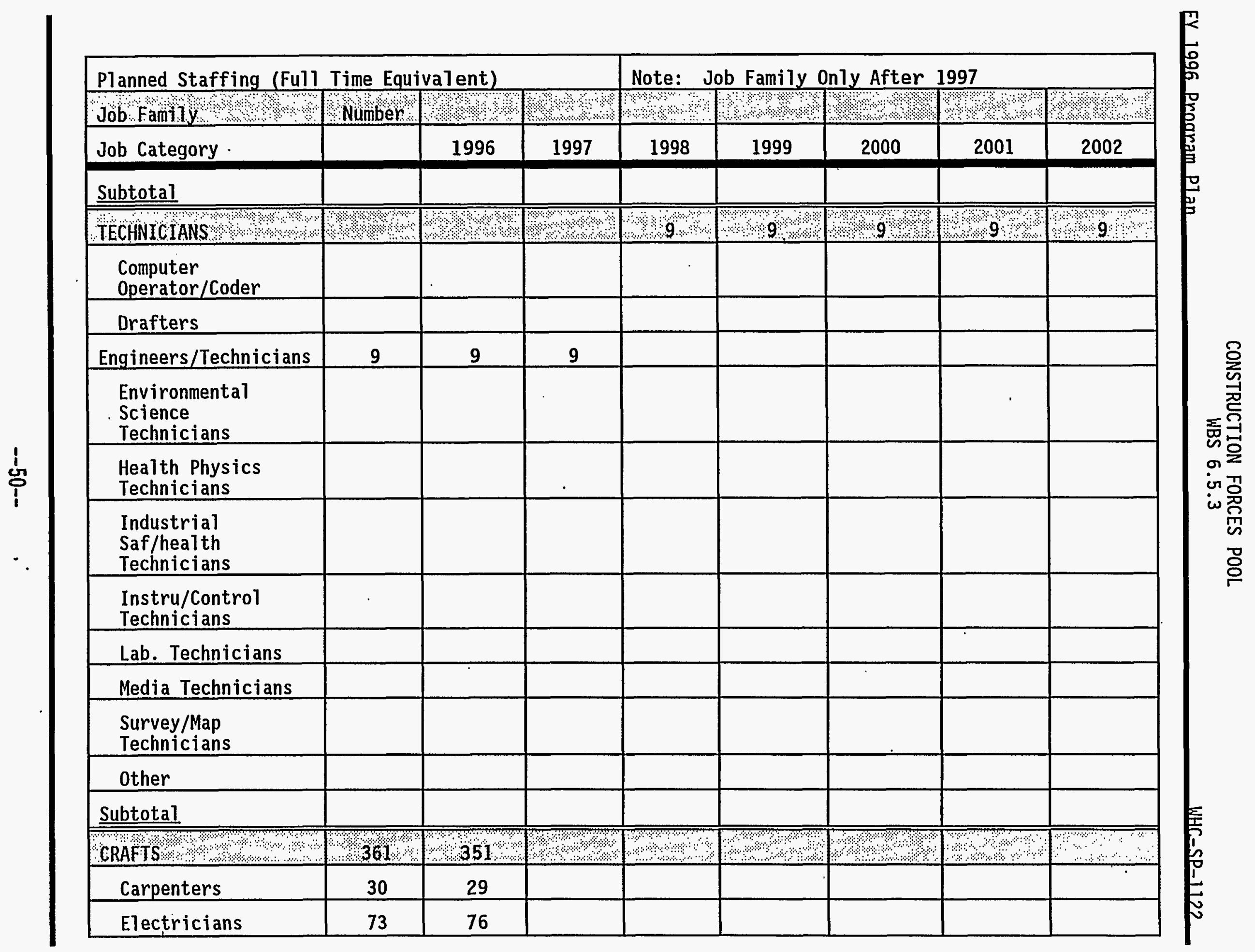




\begin{tabular}{|c|c|c|c|c|c|c|c|c|}
\hline \multicolumn{4}{|c|}{ Planned Staffing (Full Time Equivalent) } & \multicolumn{5}{|c|}{ Note: Job Family Only After 1997} \\
\hline Job Family & Nümber & अै। & ৪४ঃ & अख़ & ४े। & ४४ ४४ & मे। & ফি \\
\hline Job Category & & 1996 & 1997 & 1998 & 1999 & 2000 & 2001 & 2002 \\
\hline \multicolumn{9}{|l|}{ HVAC } \\
\hline \multicolumn{9}{|l|}{ Machinists } \\
\hline Masons & 2 & 2 & & & & & & \\
\hline Millwrights & 3 & 3 & & & & & & \\
\hline Painters & 17 & 21 & & & & & & \\
\hline Plmbrs/Pipefitters & 74 & 72 & & & & & & \\
\hline $\begin{array}{l}\text { Struct/Meta1 } \\
\text { Workers }\end{array}$ & 32 & 33 & & & & & & \\
\hline \multicolumn{9}{|l|}{$\begin{array}{l}\text { Vehicl/Mobile } \\
\text { Equip. } \\
\text { Mechanics }\end{array}$} \\
\hline \multicolumn{9}{|l|}{ Welders } \\
\hline Other & 130 & 115 & & & & & & \\
\hline \multicolumn{9}{|l|}{ Subtotal } \\
\hline OPERATORS & $1 \%$ & $\%: 17 \%$ & ४ै। : & ४ै। & \%ै० & $1 \% \%+\%$ & १ै। & ४ि४४ \\
\hline Lt. Vehicle Drivers & 17 & 17 & & & & & & \\
\hline \multicolumn{9}{|l|}{$\begin{array}{l}\text { Material Moving } \\
\text { Equip. }\end{array}$} \\
\hline \multicolumn{9}{|l|}{ Nuclear Plant } \\
\hline \multicolumn{9}{|l|}{$\begin{array}{l}\text { Utilities Waste } \\
\text { Proces }\end{array}$} \\
\hline \multicolumn{9}{|l|}{ other } \\
\hline Subtotal & & & & & & & & \\
\hline
\end{tabular}




\begin{tabular}{|c|c|c|c|c|c|c|c|c|}
\hline \multicolumn{4}{|c|}{ Planned Staffing (Full Time Equivalent) } & \multicolumn{5}{|c|}{ Note: Job Family Only After 1997} \\
\hline Job Family & Number: & ४४\% & एै? & अथ। & अेष & ४०४०8 & ४े: & ओ। \\
\hline Job Category & & 1996 & 1997 & 1998 & 1999 & 2000 & 2001 & 2002 \\
\hline $\begin{array}{l}\text { ABOR \& GENERAL } \\
\text { WORKERS }\end{array}$ & $8 \%$ & ४ै? & ?ै? & अै? & अै० & ${ }^{2}+2$ & मै०े? & अै। \\
\hline \multicolumn{9}{|l|}{ Firefighters } \\
\hline \multicolumn{9}{|l|}{ Food Service } \\
\hline \multicolumn{9}{|l|}{ Hand/Help Lab Gen } \\
\hline \multicolumn{9}{|l|}{$\begin{array}{l}\text { Hand/Help Lab } \\
\text { Special }\end{array}$} \\
\hline \multicolumn{9}{|l|}{ Janitors/Cleaners } \\
\hline \multicolumn{9}{|l|}{ Laundry Workers } \\
\hline \multicolumn{9}{|l|}{ Security Guards } \\
\hline \multicolumn{9}{|l|}{ other } \\
\hline \multicolumn{9}{|l|}{ Subtotal } \\
\hline TOTAL FTES & & & & & & & & \\
\hline
\end{tabular}




\section{INDEX}

1.0 DIRECT POOL - NON MANUAL

2.0 DIRECT POOL - NON MANUAL OVERVIEW (6.5.4)

2.1 Mission

2.2 Assumptions

2.3 Constraints

3.0 TECHNICAL REQUIREMENT BASELINE

3.1 Work Breakdown Structure

3.2 Work Breakdown

3.1.1 Estimating (6.5.4.1)

3.1.2 Project Planning and Control (6.5.4.2)

3.1.3 Construction Services (6.5.4.3)

3.1.4 Engineering (6.5.4.4)

3.1.5 TWRS (6.5.4.5).

3.1.6 SNF (6.5.4.6)

3.1.7 PNL Landlord, Engineering and Construction Services (6.5.4.7)

3.1.8 Transition Projects/Site Engineering \& Construction Services (6.5.4.8)

4.0 SCHEDULE BASELINE

4.1 Product Deliverables/Milestone List

5.0 COST BASELINE SUMMARY

$5.1 \quad$ Cost Baseline

5.2 Planned Staffing Profile 


\subsection{DIRECT POOL - NON MANUAL}

The purpose of the pool is to accumulate all labor costs that primarily perform engineering, project management, and construction management services.

\subsection{DIRECT POOL - NON MANUAL OVERVIEW (6.5.4)}

\subsection{Mission/Vision}

The mission statement will be included within the individual Work Breakdown Structure narratives.

\subsection{Assumptions}

The following are key assumptions used to develop goals and strategic (building blocks): Reduction of cost is paramount to success and competitive posturing. Outsourcing of work will be conducted on a cost/performance advantage basis, funding and staffing levels will be adequate, re-engineering initiatives will be fully supported, award fee and incentive fee criteria will be established, cost savings programs will be supported, work will comply with current regulations and guidance, and the Voluntary Protection Program will continue.

\subsection{Constraints}

Items that may impede achievements of goals and strategies are as follows: privatization and outsourcing of services, funding support to ICF KH must be adequate for the scope required by DOE, contradictory DOE missions, scheduling/cost software programs changed, Prioritization of work due to budget constraints, and continued company reorganizations. 


\section{DIRECT POOL - NON-MANUAL}

WBS 6.5 .4

FY 1996 Program Plan

3.0 TECHNICAL REQUIREMENTS BASELINE

3.1 Work Breakdown Structure

3.2 Work Breakdown 


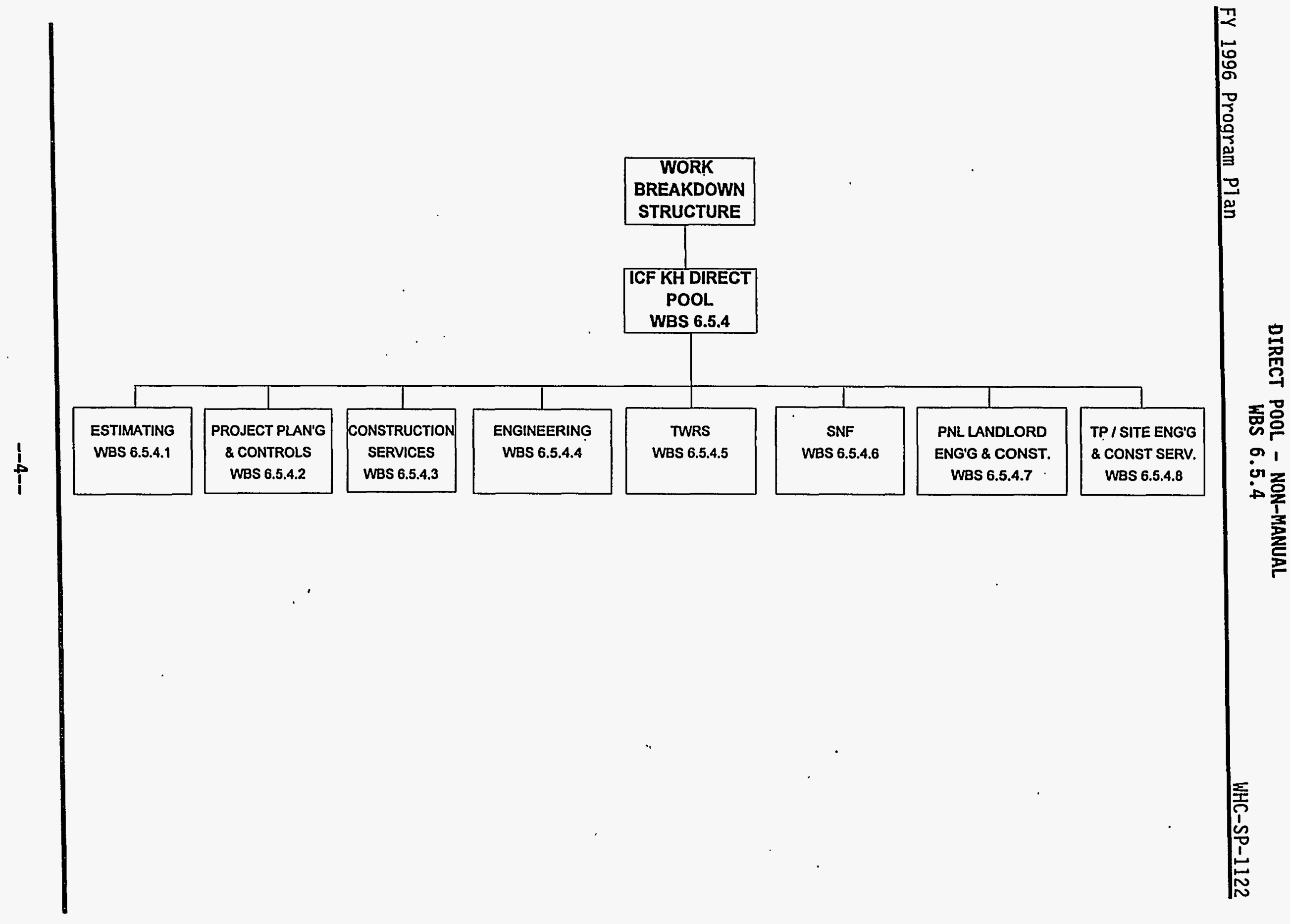


RECT POOL - NON-MANUAL (6.5.4)

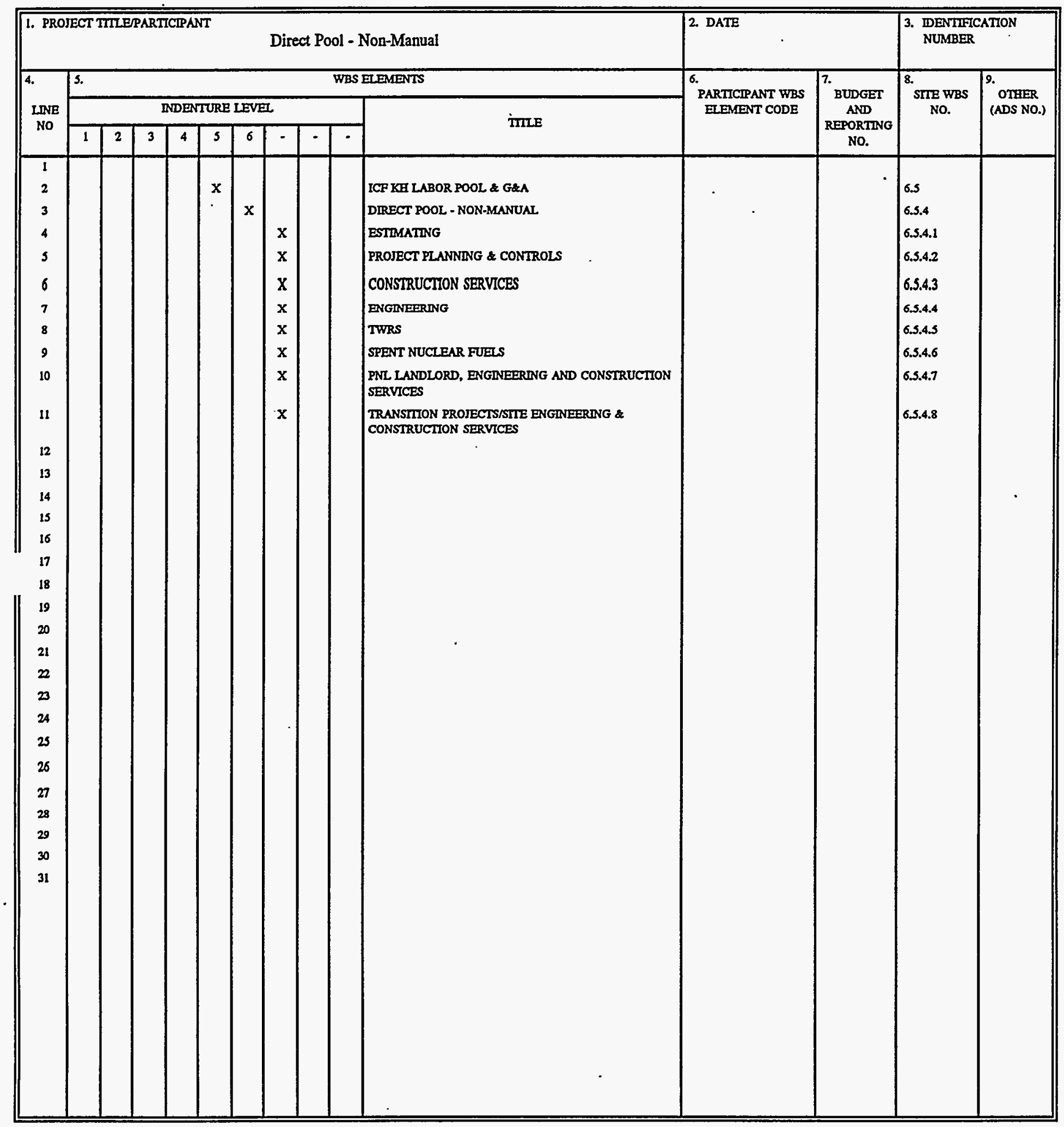




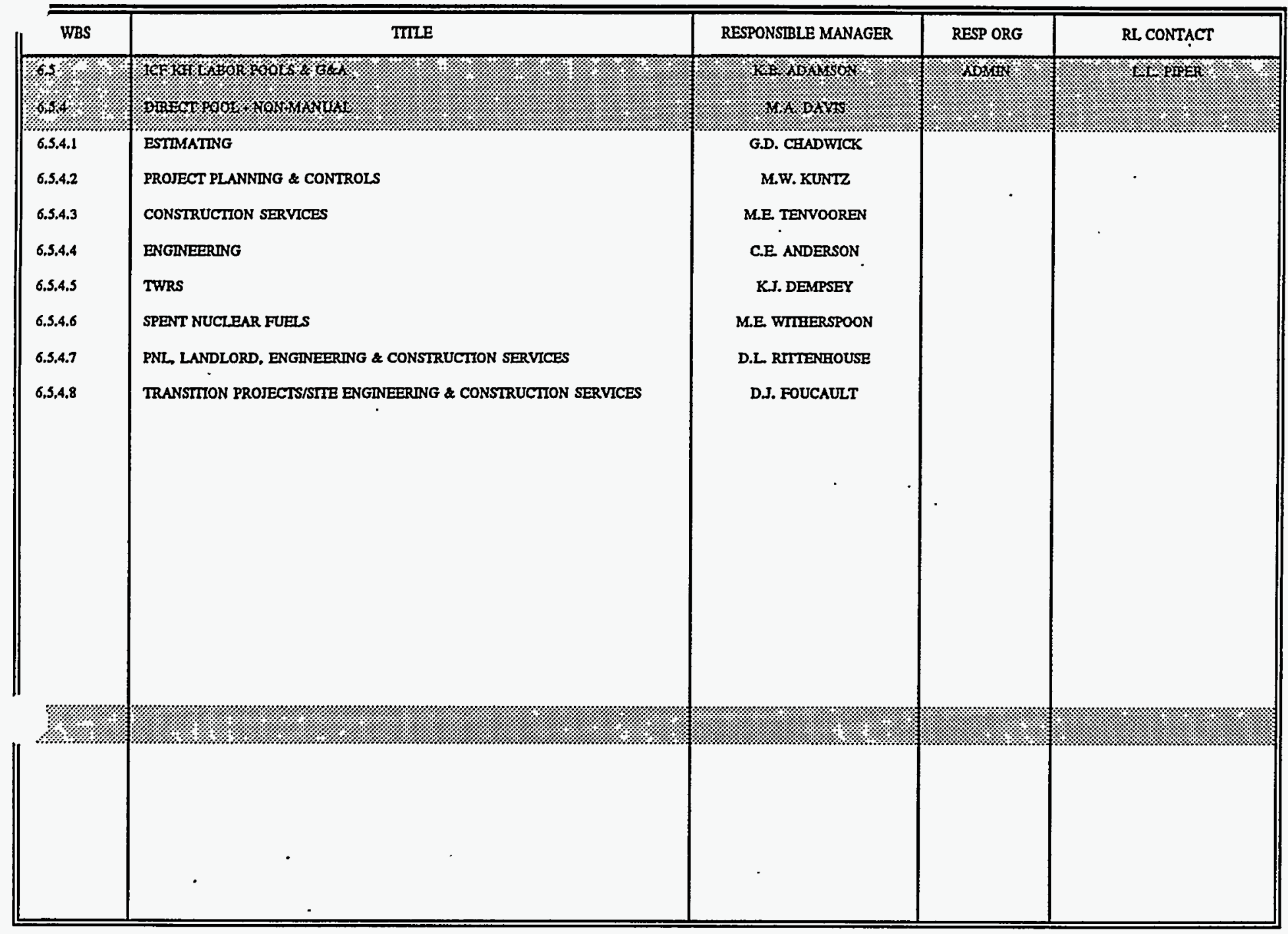


Table 3-1. FY 1996 SSPP DIRECT POOL - NON-MANUAL WBS Dictionary (6.5.4)

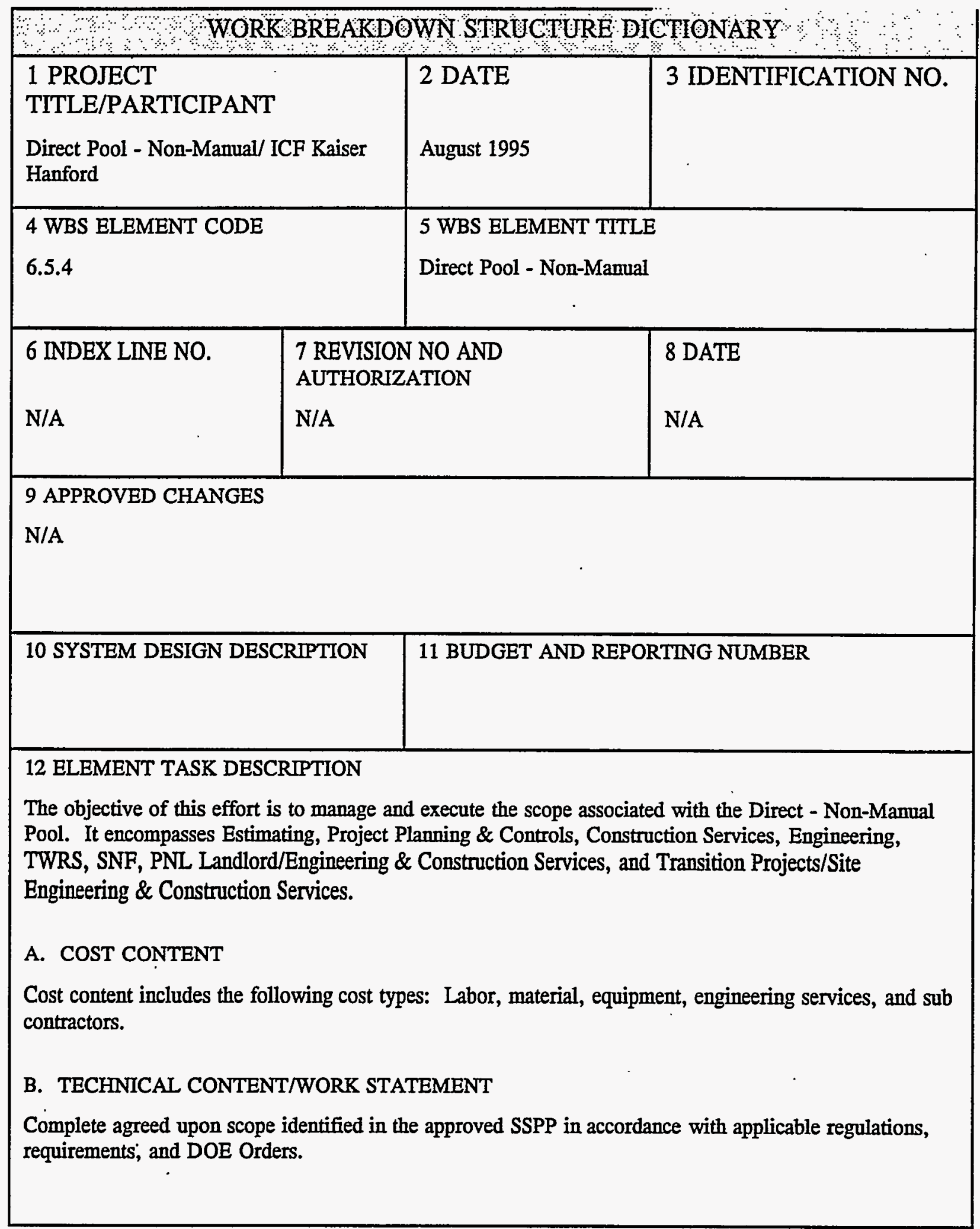




\subsubsection{Estimating (WBS 6.5.4.1)}

\section{Mission}

The ICF Kaiser Hanford Estimating organization services the current capital construction efforts, along with meeting current and future programmatic needs in support of the Hanford Clean up Mission.

\section{Products/Services Provided}

The Estimating organization provides the following:

- Capital Construction Services: Provides the estimating services normal7y associated with project under authorization (i.e. from conceptual design forward). The services include the preparation of conceptual cost estimates, preliminary design estimates, definitive design cost estimates, fair cost estimates, current working cost estimates, change request cost estimates and engineering design and inspection cost estimates. Other services performed are: Peer reviews; VE Studies; and "other" project cost definition.

- Programmatic Cost Services: Provides estimating services normally associated with Programmatic/Operation activities. Sample services provided are: Preparation of Study Estimates (i.e. Order of Magnitude, Engineering Studies, etc.), Activity Based Cost Estimating activities (i.e. Baseline and ReBaseline ADS cost data for the five year plan ADS's, Multi-Year Program Plans, and Fiscal Year Work Plans) and Life Cycle Cost Analysis.

\section{Primary Customers}

The Primary Customers of the Estimating Organization are: ICF Kaiser Hanford, Westinghouse Hanford Company, Pacific Northwest Laboratory, and U. S. Department of Energy (RL). 


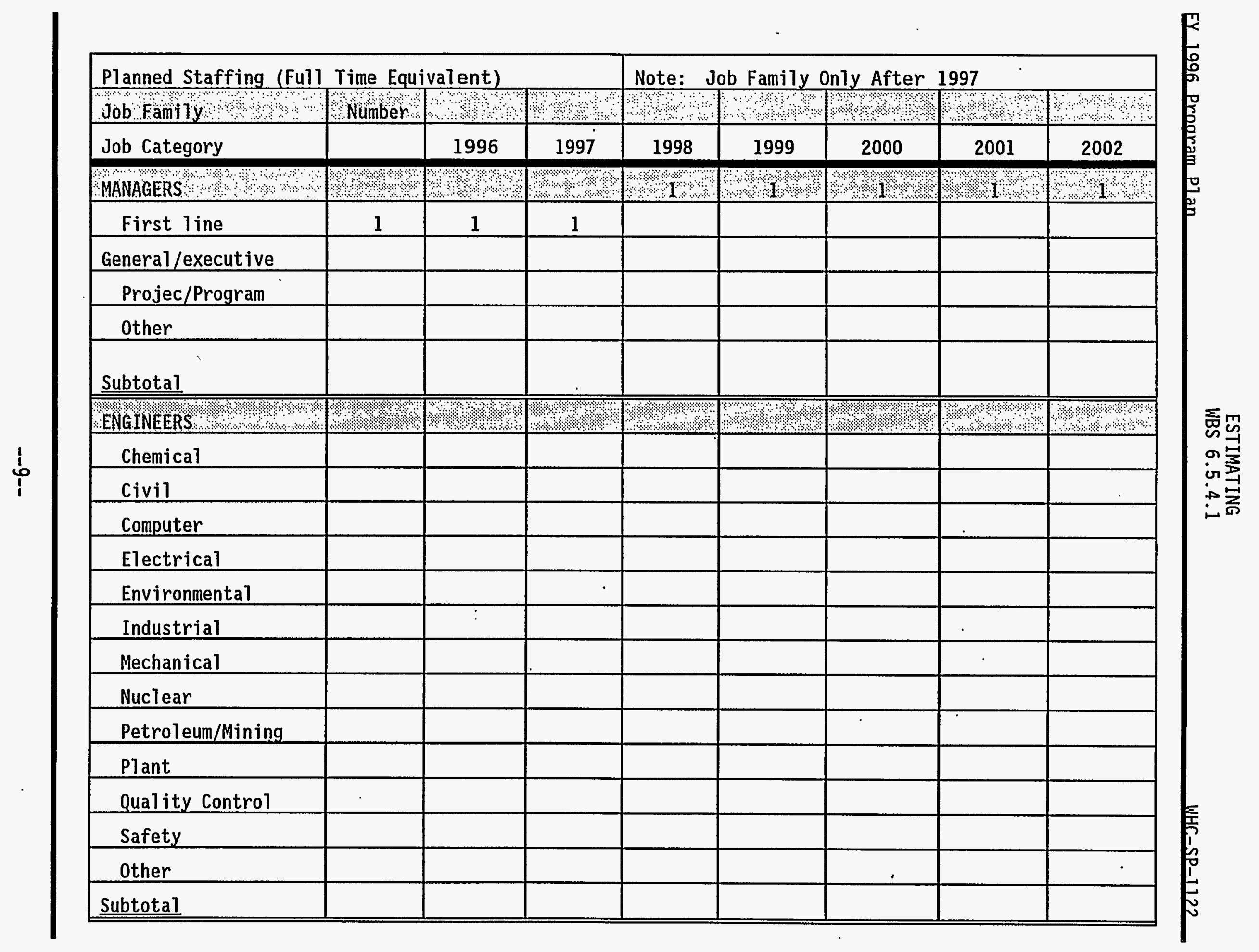




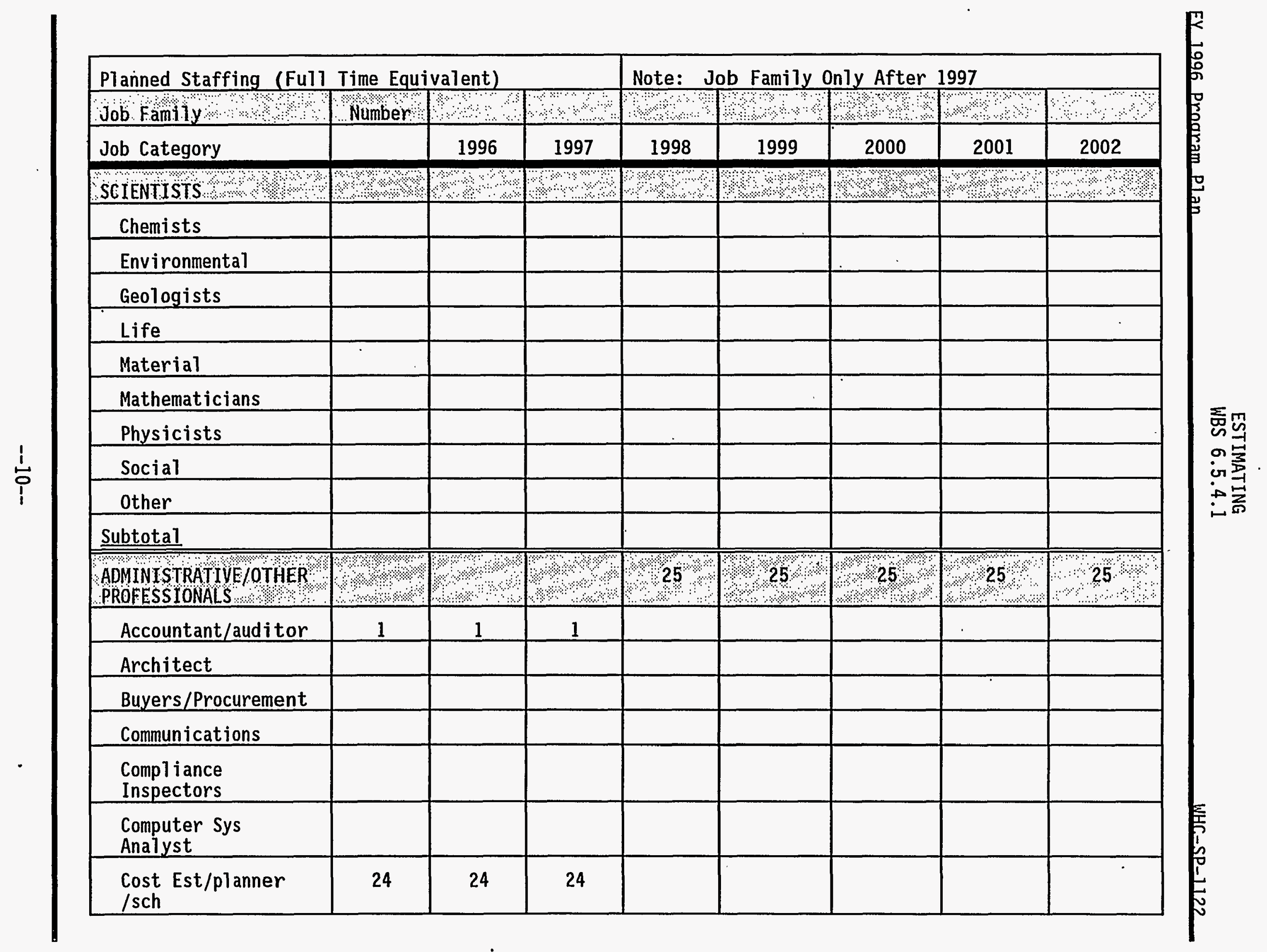




\begin{tabular}{|c|c|c|c|c|c|c|c|c|}
\hline \multicolumn{9}{|c|}{ Planned Staffing (Full Time Equivalent) } \\
\hline Job family & Number: & 3 & अै। & в. & आ०े & ४ै० & अे & 3 \\
\hline Job Category & & 1996 & 1997 & 1998 & 1999 & 2000 & 2001 & 2002 \\
\hline \multicolumn{9}{|l|}{ Health Physics } \\
\hline \multicolumn{9}{|l|}{ Industrial Hygiene } \\
\hline \multicolumn{9}{|l|}{ Lawyers } \\
\hline \multicolumn{9}{|l|}{$\begin{array}{l}\text { Personnel/labor } \\
\text { Relations }\end{array}$} \\
\hline \multicolumn{9}{|l|}{ Physicians } \\
\hline \multicolumn{9}{|l|}{$\begin{array}{l}\text { Physician } \\
\text { Assistant/Nurse }\end{array}$} \\
\hline \multicolumn{9}{|l|}{$\begin{array}{l}\text { Safeguard \& } \\
\text { Security }\end{array}$} \\
\hline \multicolumn{9}{|l|}{$\begin{array}{l}\text { Tech Writers \& } \\
\text { Editors }\end{array}$} \\
\hline \multicolumn{9}{|l|}{ Trainers } \\
\hline \multicolumn{9}{|l|}{ other } \\
\hline \multicolumn{9}{|l|}{ Subtotal } \\
\hline $\begin{array}{l}\text { GENERAL } \\
\text { ADMIN/SECRETARY/GLERK }\end{array}$ & अे? & \%? ? & भै? & \%? & $\mathrm{P}_{2}$ & \% & ? 2 & ${ }_{2}$ \\
\hline \multicolumn{9}{|l|}{ Admin Assistant } \\
\hline Office Clerk (Gen) & 1 & 1 & 1 & & & & & \\
\hline \multicolumn{9}{|l|}{$\begin{array}{l}\text { Office Clerk } \\
\text { (Special) }\end{array}$} \\
\hline Secretaries & 1 & 1 & 1 & & & & & \\
\hline \multicolumn{9}{|l|}{$\begin{array}{l}\text { Typist/Word } \\
\text { Processor }\end{array}$} \\
\hline Other & & & & & & & & \\
\hline
\end{tabular}




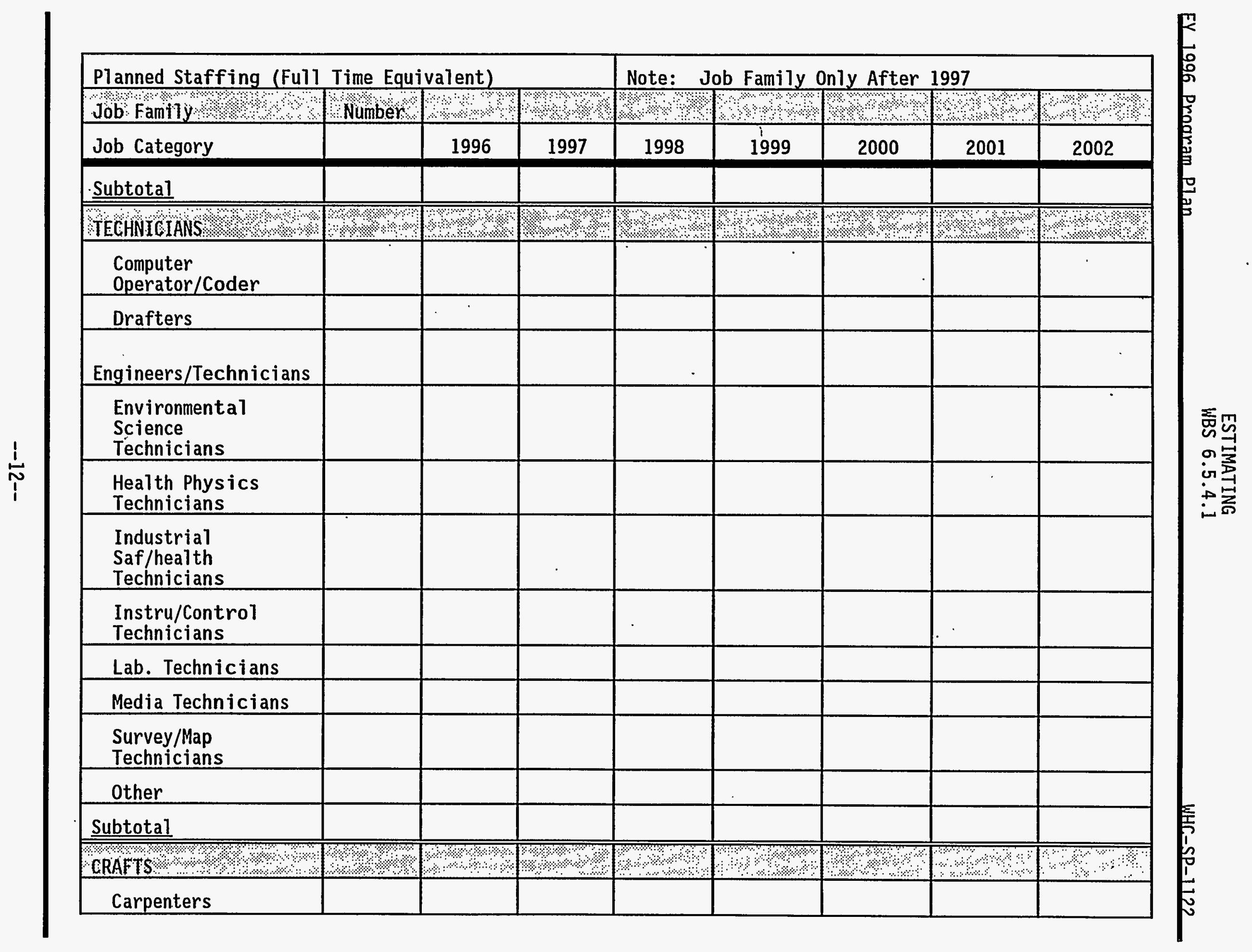




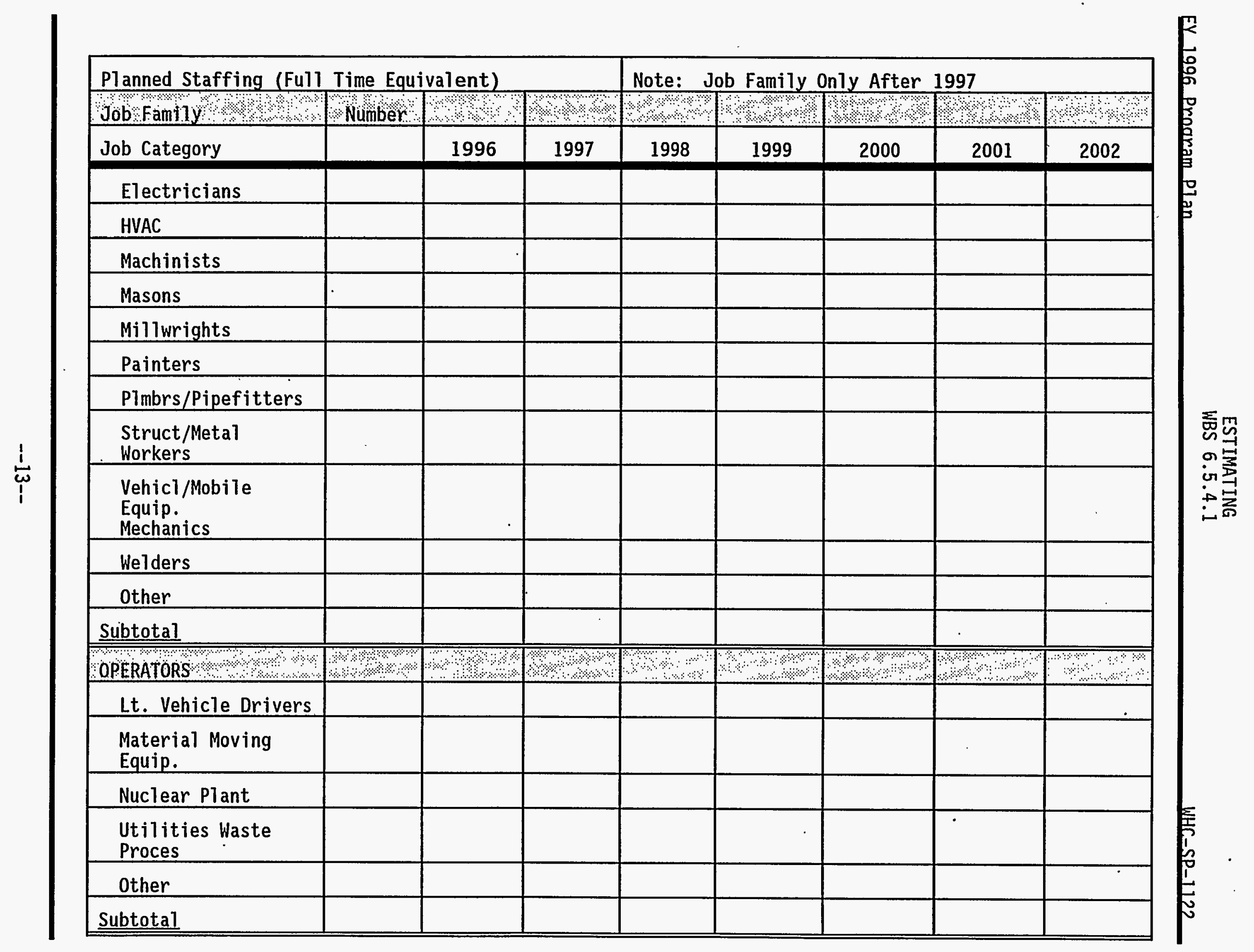




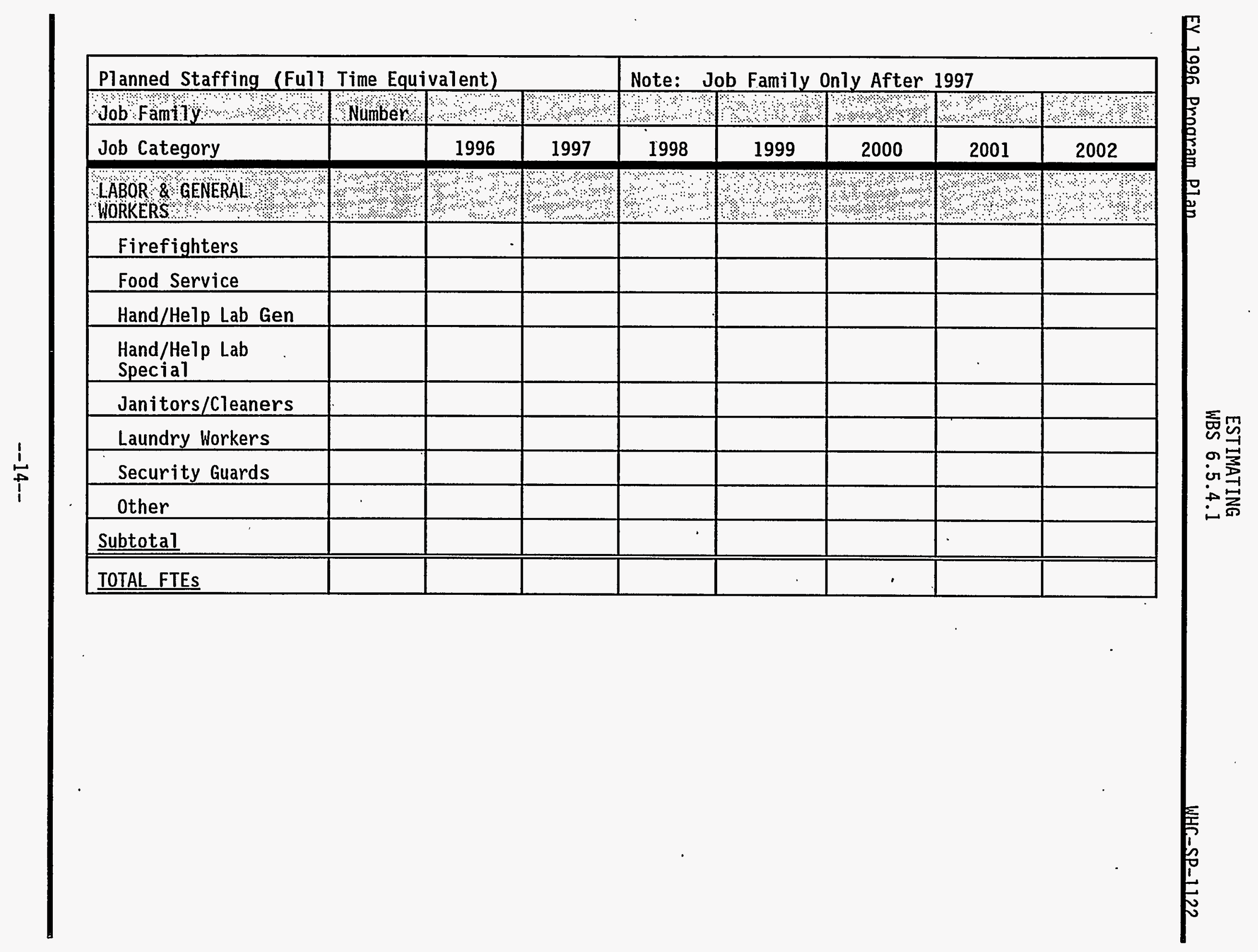


3.1.2 Project P1anning and Controls (WBS 6.5.4.2)

Mission

The mission of the Project Planning and Controls Department is to provide cost, schedule and administrative support to the ICF KH Project Managers.

\section{Products/Services Provided}

The Project.Planning and Controls organization provides the following: Work Vó7ume Forecast, TPA Agreement reporting, Award Fee reporting, Executive Status reporting, Site Support Program PIans, Site Management System reporting, Work Order set-up/maintenance/closeout, Senior Management reviews, Basic Ordering Agreement contract administration, KEMS/KFS upgrades and maintenance, procedure development, Manpower Status reports, Forecast vs. Actual reporting, and Software control.

Primary Customers

The primary customers of the Project Planning and Controls are: ICF Kaiser Hanford, ICF Kaiser Corporate, Westinghouse Hanford Company, Pacific Northwest Laboratory, and U.S. Department of Energy (RL). 


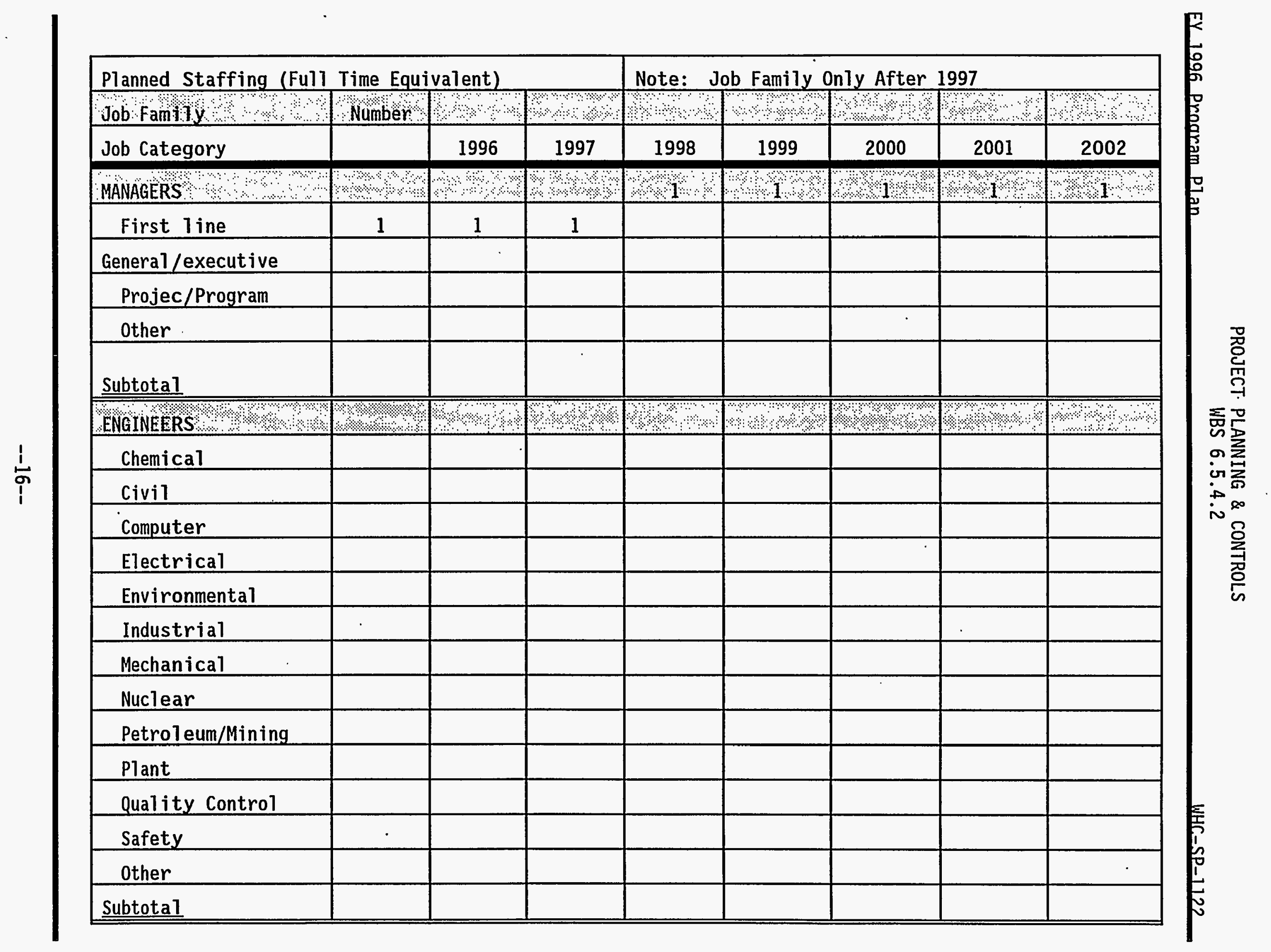




\begin{tabular}{|c|c|c|c|c|c|c|c|c|}
\hline \multicolumn{4}{|c|}{ Planned Staffing (Full Time Equivalent) } & \multicolumn{5}{|c|}{ Note: Job Family Only After 1997} \\
\hline Job Famlly & Number: & अ४। & अ ஃ: & 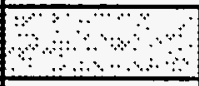 & ए४े & ४४४४? & अथ & $\sqrt{4+4}$ \\
\hline Job Category & & 1996 & 1997 & 1998 & 1999 & 2000 & 2001 & 2002 \\
\hline SCIENTISTS & अ, \% & +ि, & ঝे: & ४ & अे & ४४ & अ० & अ: \\
\hline Chemists & & & & & & & & \\
\hline Environmental & & & & & & & & \\
\hline Geologists & & & & & & & & \\
\hline Life & & & & & & & & \\
\hline Material & & & & & & & & \\
\hline Mathematicians & & & & & & & & \\
\hline Physicists & & & & & & & & \\
\hline Social & & & & & & & & \\
\hline Other & & & & & & & & \\
\hline Subtotal & & & & & & & & \\
\hline $\begin{array}{l}\text { ADMINISTRATIVE/OTHER } \\
\text { PROFESSIONALS }\end{array}$ & \%थ थ & शै? & \%थे \% & ४ै। & ?+1 = & \% $11 \%$ & ?1\%? & $1 \%$ \\
\hline Accountant/auditor & 1 & 1 & 1 & & & & & \\
\hline Architect & & & & & & & & \\
\hline Buyers/Procurement & & & & & & & & \\
\hline Communications & & & & & & & & \\
\hline $\begin{array}{l}\text { Compliance } \\
\text { Inspectors }\end{array}$ & & & & & & & & \\
\hline $\begin{array}{l}\text { Computer Sys } \\
\text { Analyst }\end{array}$ & & & & & & & & \\
\hline $\begin{array}{l}\text { Cost Est/planner } \\
\text { /sch }\end{array}$ & & & & & & & & \\
\hline
\end{tabular}




\begin{tabular}{|c|c|c|c|c|c|c|c|c|}
\hline \multicolumn{9}{|c|}{ Planned Staffing (Fu 11 Time Equivalent) } \\
\hline Job Famf $1 y$ s & Nümber & ४ি & अे। & अ? & ४४४ & $18 \%$ & एै। थ. & अ४। \\
\hline Job Category & & 1996 & 1997 & 1998 & 1999 & 2000 & 2001 & 2002 \\
\hline \multicolumn{9}{|l|}{ Health Physics } \\
\hline \multicolumn{9}{|l|}{ Industrial Hygiene } \\
\hline \multicolumn{9}{|l|}{ Lawyers } \\
\hline \multicolumn{9}{|l|}{$\begin{array}{l}\text { Personnel/labor } \\
\text { Relations }\end{array}$} \\
\hline \multicolumn{9}{|l|}{ Physicians } \\
\hline \multicolumn{9}{|l|}{$\begin{array}{l}\text { Physician } \\
\text { Assistant/Nurse }\end{array}$} \\
\hline \multicolumn{9}{|l|}{$\begin{array}{l}\text { Safeguard \& } \\
\text { Security }\end{array}$} \\
\hline \multicolumn{9}{|l|}{$\begin{array}{l}\text { Tech Writers \& } \\
\text { Editors }\end{array}$} \\
\hline \multicolumn{9}{|l|}{ Trainers } \\
\hline \multicolumn{9}{|l|}{ Other } \\
\hline \multicolumn{9}{|l|}{ Subtota1 } \\
\hline $\begin{array}{l}\text { GENERAL } \\
\text { ADMIN/SECRETARY/CLERK }\end{array}$ & अे? & $\%$ & अे? & 2 & अे & \%? & कि & 2 \\
\hline \multicolumn{9}{|l|}{ Admin Assistant } \\
\hline Office Clerk (Gen) & 2 & 2 & 2 & & & & & \\
\hline \multicolumn{9}{|l|}{$\begin{array}{l}\text { Office Clerk } \\
\text { (Special) }\end{array}$} \\
\hline \multicolumn{9}{|l|}{ Secretaries } \\
\hline \multicolumn{9}{|l|}{$\begin{array}{l}\text { Typist/Word } \\
\text { Processor }\end{array}$} \\
\hline Other & & & & & & & & \\
\hline
\end{tabular}




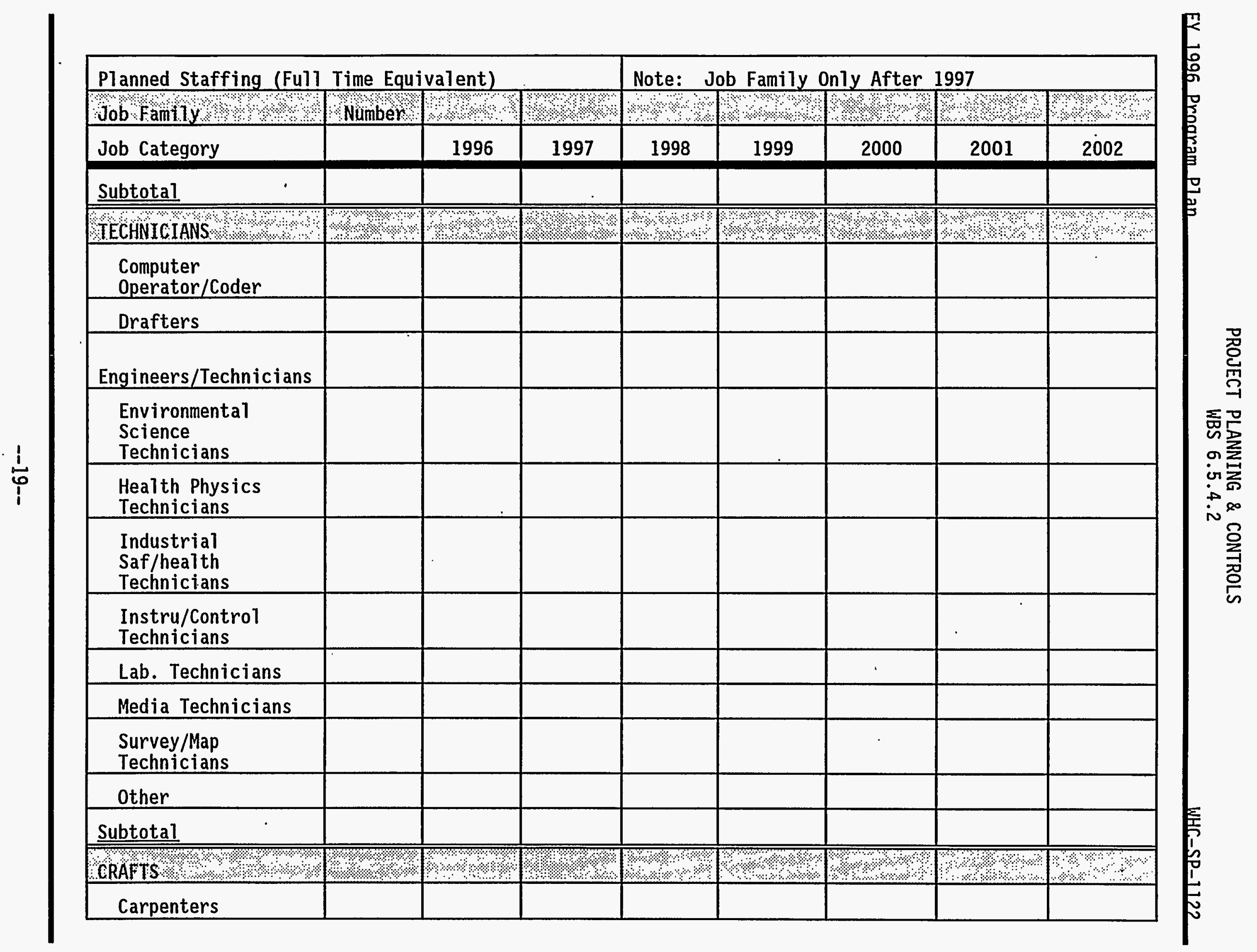




\begin{tabular}{|c|c|c|c|c|c|c|c|c|}
\hline \multicolumn{4}{|c|}{ Planned Staffing (Ful1 Time Equivalent) } & \multicolumn{5}{|c|}{ Note: Job Family Only After 1997} \\
\hline Job Family & Nümber & अल & \%) & Oै? & अ०: & ४৷ & \%) & ।ি: \\
\hline Job Category & & 1996 & 1997 & 1998 & 1999 & 2000 & 2001 & 2002 \\
\hline \multicolumn{9}{|l|}{ Electricians } \\
\hline \multicolumn{9}{|l|}{ HVAC } \\
\hline \multicolumn{9}{|l|}{ Machinists } \\
\hline \multicolumn{9}{|l|}{ Masons } \\
\hline \multicolumn{9}{|l|}{ Millwrights } \\
\hline \multicolumn{9}{|l|}{ Painters } \\
\hline \multicolumn{9}{|l|}{ Plmbrs/Pipefitters } \\
\hline \multicolumn{9}{|l|}{$\begin{array}{l}\text { Struct/Metal } \\
\text { Workers }\end{array}$} \\
\hline \multicolumn{9}{|l|}{$\begin{array}{l}\text { Vehicl/Mobile } \\
\text { Equip. } \\
\text { Mechanics }\end{array}$} \\
\hline \multicolumn{9}{|l|}{ Welders } \\
\hline \multicolumn{9}{|l|}{ Other } \\
\hline \multicolumn{9}{|l|}{ Subtotal } \\
\hline OPERATORS & अ०० & ২০ & ?ै? & अ०० & अै। & ४ ४ & अें & ब \\
\hline \multicolumn{9}{|l|}{ Lt. Vehicle Drivers } \\
\hline \multicolumn{9}{|l|}{$\begin{array}{l}\text { Material Moving } \\
\text { Equip. }\end{array}$} \\
\hline \multicolumn{9}{|l|}{ Nuclear Plant } \\
\hline \multicolumn{9}{|l|}{$\begin{array}{l}\text { Utilities Waste } \\
\text { Proces }\end{array}$} \\
\hline \multicolumn{9}{|l|}{ Other } \\
\hline Subtotal & & & & & & & & \\
\hline
\end{tabular}




\begin{tabular}{|c|c|c|c|c|c|c|c|c|}
\hline \multicolumn{4}{|c|}{ Planned Staffing (Full Time Equivalent) } & \multicolumn{5}{|c|}{ Note: Job Family Only After 1997} \\
\hline Job Family & Number & \& & ४४\% & 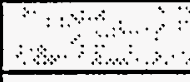 & अ४४ & अथे & 2 & , « \\
\hline Job Category & & 1996 & 1997 & 1998 & 1999 & 2000 & 2001 & 2002 \\
\hline $\begin{array}{l}\angle A B O R \& \text { GENERAL } \\
\text { WORKERS }\end{array}$ & \%ै? & +ै। & \%ै? & ४ैै & अे? & ४ै० & \%ै। & s. \\
\hline Firefighters & & & & & & & & \\
\hline Food Service & & & & & & & & \\
\hline Hand/Help Lab Gen & & & & & & & & \\
\hline $\begin{array}{l}\text { Hand/Help Lab } \\
\text { Special }\end{array}$ & & & & & & & & \\
\hline Janitors/Cleaners & & & & & & & & \\
\hline Laundry Workers & & & & & & & & \\
\hline Security Guards & & & & & & & & \\
\hline Other & & & & & & & & \\
\hline Subtotal & & & & & & & & \\
\hline TOTAL FTES & & & & & & & & \\
\hline
\end{tabular}




\subsubsection{Construction Services (WBS 6.5.4.3)}

\section{Mission}

The Construction Services Division sets standards and processes for construction through implementing procedures to ensure consistency in the application of Company Policy and unified effort in achieving performance expectations.

\section{Products/Services Provided}

The Construction Services division provides the Sub Contractor Industrial Safety, Health and Environmental Programs, Construction Divisions Self Assessement and Corrective Action coordination as well as the coordination of Construction Training requirements identification and completion. Provides for liason between construction trades, management and the public at large. Coordination of self improvement programs including VPP implementation. Also provides for the control distribution of configuration documentation.

\section{Primary Customer}

The primary customers of the Construction Services Division are: ICF Kaiser Hanford, Westinghouse Hanford Company, Pacific Northwest Laboratory, and U.S. Department of Energy (RL). 


\begin{tabular}{|c|c|c|c|c|c|c|c|c|}
\hline \multicolumn{4}{|c|}{ Planned Staffing (Ful1 Time Equivalent) } & \multicolumn{5}{|c|}{ Note: Job Family Only After 1997} \\
\hline Job Famhy $)$ & Number & अ० & \%? & ४४४ & ঋ४४। & \%०० & ४४४: & \%ै। \\
\hline Job Category & & 1996 & 1997 & 1998 & 1999 & 2000 & 2001 & 2002 \\
\hline MANAGERS $+\%$ & ঋ+: & +3 & \%॰ & ४3: & ?ै, 3 & $2 \% 3$ & 43 & $1_{3}$ \\
\hline First line & 2 & 2 & 2 & & & & & \\
\hline General/executive & 1 & 1 & 1 & & & & & \\
\hline \multicolumn{9}{|l|}{ Projec/Program } \\
\hline \multicolumn{9}{|l|}{ Other } \\
\hline \multicolumn{9}{|l|}{ Subtotal } \\
\hline ENGINEERS & \%? & $\%$ & $\%$ & 10 & १० $10 \%$ & 2.10 & 10. & +10 \\
\hline \multicolumn{9}{|l|}{ Chemical } \\
\hline Civil & 2 & 2 & 2 & & . & & & \\
\hline \multicolumn{9}{|l|}{ Computer } \\
\hline \multicolumn{9}{|l|}{ Electrical } \\
\hline \multicolumn{9}{|l|}{ Environmental } \\
\hline \multicolumn{9}{|l|}{ Industrial } \\
\hline Mechanical & & & & $\cdot$ & & & & \\
\hline \multicolumn{9}{|l|}{ Nuclear } \\
\hline \multicolumn{9}{|l|}{ Petroleum/Mining } \\
\hline Plant & 6 & 6 & 6 & & & & & \\
\hline \multicolumn{9}{|l|}{ Quality Control } \\
\hline Safety & 2 & 2 & 2 & & & & & \\
\hline Other & & & & & & & & . \\
\hline Subtotal & & & & & & & & \\
\hline
\end{tabular}




\begin{tabular}{|c|c|c|c|c|c|c|c|c|}
\hline \multicolumn{4}{|c|}{ Planned Staffing (Full Time Equivalent) } & \multicolumn{5}{|c|}{ Note: Job Family Only After 1997} \\
\hline Job family ${ }^{\prime}$ & Number & ४४४ & 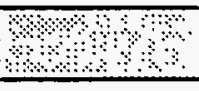 & अ: & ए। & \%थ। & ४ै। & ४ें \\
\hline Job Category & & 1996 & 1997 & 1998 & 1999 & 2000 & 2001 & 2002 \\
\hline \multicolumn{9}{|c|}{ 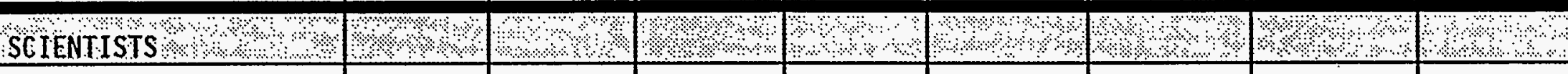 } \\
\hline \multicolumn{9}{|c|}{\begin{tabular}{|l|l|l|l|l|l|l|l|l|} 
Chemists & & & & & & & & \\
\end{tabular}} \\
\hline \multicolumn{9}{|l|}{ Environmental } \\
\hline \multicolumn{9}{|l|}{ Geologists } \\
\hline \multicolumn{9}{|l|}{ Life } \\
\hline \multicolumn{9}{|l|}{ Material } \\
\hline \multicolumn{9}{|l|}{ Mathematicians } \\
\hline \multicolumn{9}{|l|}{ Physicists. } \\
\hline \multicolumn{9}{|l|}{ Social } \\
\hline \multicolumn{9}{|l|}{ other } \\
\hline \multicolumn{9}{|l|}{ Subtotal } \\
\hline $\begin{array}{l}\text { ADMINISTRATIVE } / O T H E R \\
\text { PROFESSTONALS }\end{array}$ & ४ै। & \%ै? & धथ \% & \%े। & अे & ४ै & & 4 \\
\hline Accountant/auditor & 2 & 2 & 2 & & & & & \\
\hline \multicolumn{9}{|l|}{ Architect } \\
\hline \multicolumn{9}{|l|}{ Buyers/Procurement } \\
\hline \multicolumn{9}{|l|}{ Communications } \\
\hline \multicolumn{9}{|l|}{$\begin{array}{l}\text { Compliance } \\
\text { Inspectors }\end{array}$} \\
\hline \multicolumn{9}{|l|}{$\begin{array}{l}\text { Computer Sys } \\
\text { Analyst }\end{array}$} \\
\hline $\begin{array}{l}\text { Cost Est/planner } \\
\text { /sch }\end{array}$ & & & & & & & & \\
\hline
\end{tabular}




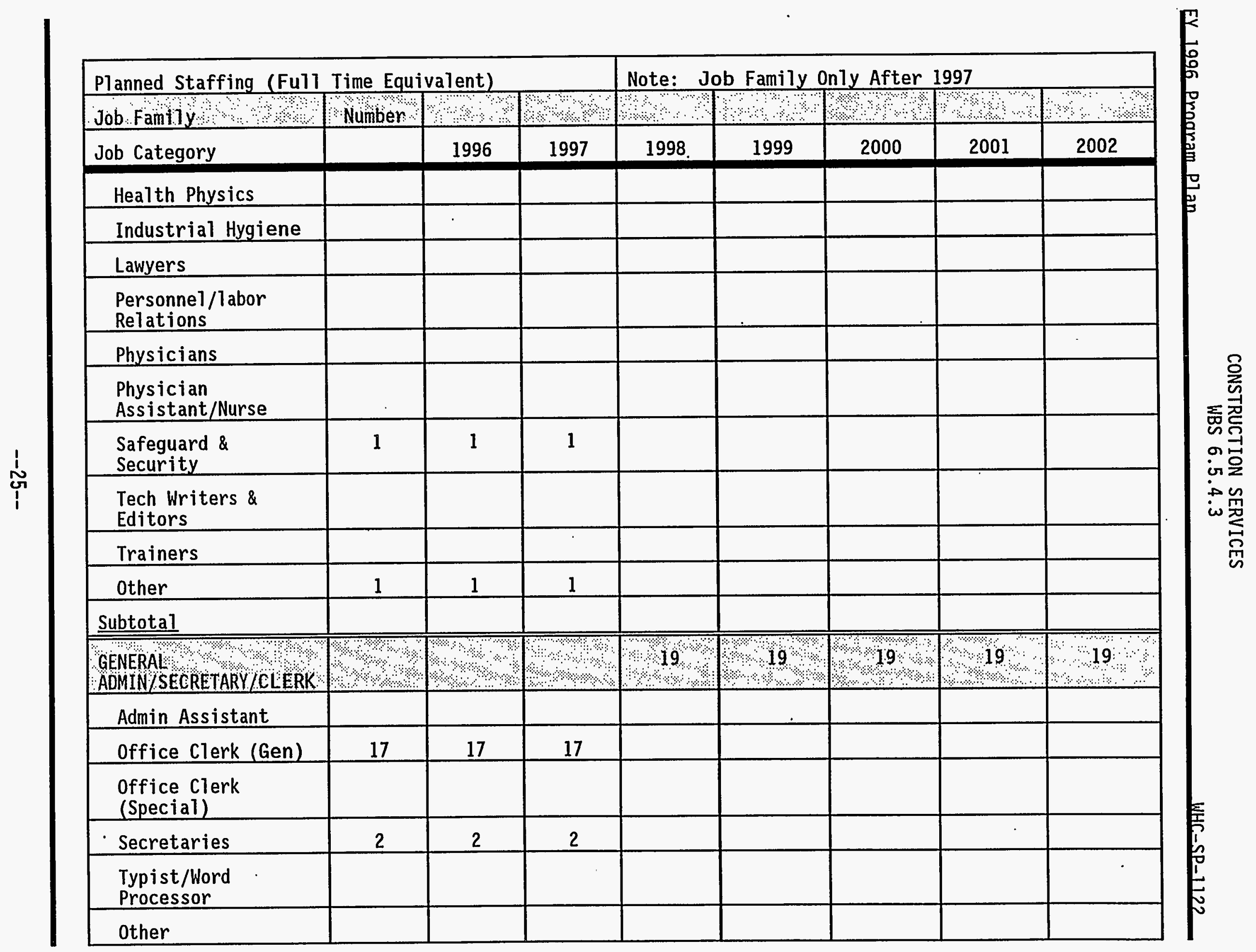




\begin{tabular}{|c|c|c|c|c|c|c|c|c|}
\hline \multicolumn{4}{|c|}{ Planned Staffing (Full Time Equivalent) } & \multicolumn{5}{|c|}{ Note: Job Family Oniy After 1997} \\
\hline Job family & Nümber & ४००: & $120 \%$ & \%थ\% & \%०४ & $10 \%$ & 14\%? & $2 \%$ \\
\hline Job Category & & 1996 & 1997 & 1998 & 1999 & 2000 & 2001 & 2002 \\
\hline \multicolumn{9}{|l|}{ Subtotal } \\
\hline TECHNICIANS & $2 \times 4$ & थथ & $\sqrt[2]{2}$ & \%? & \%? & (- & $4 \%$ & 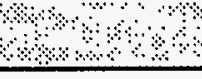 \\
\hline \multicolumn{9}{|c|}{\begin{tabular}{l|l|l|l} 
Computer & & & \\
Operator/Coder & & & \\
\end{tabular}} \\
\hline \multicolumn{9}{|l|}{ Drafters } \\
\hline \multicolumn{9}{|l|}{ Engineers/Technicians } \\
\hline \multicolumn{9}{|l|}{$\begin{array}{l}\text { Environmental } \\
\text { Science } \\
\text { Technicians }\end{array}$} \\
\hline \multicolumn{9}{|l|}{$\begin{array}{l}\text { Health Physics } \\
\text { Technicians }\end{array}$} \\
\hline \multicolumn{9}{|l|}{$\begin{array}{l}\text { Industrial } \\
\text { Saf/health } \\
\text { Technicians } \\
\end{array}$} \\
\hline \multicolumn{9}{|l|}{$\begin{array}{l}\text { Instru/Contro1 } \\
\text { Technicians }\end{array}$} \\
\hline \multicolumn{9}{|l|}{ Lab. Technicians } \\
\hline \multicolumn{9}{|l|}{ Media Technicians } \\
\hline \multicolumn{9}{|l|}{$\begin{array}{l}\text { Survey/Map } \\
\text { Technicians }\end{array}$} \\
\hline \multicolumn{9}{|l|}{ Other } \\
\hline \multicolumn{9}{|l|}{ Subtotal } \\
\hline CRAFTS,$\cdots$, & $4 \div 2$ & \$? & 1.2. & 2.2 & \%० & 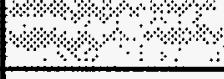 & 2.2. & 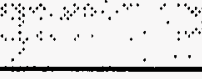 \\
\hline \multicolumn{9}{|l|}{ Carpenters } \\
\hline Electricians & & & & & & & & \\
\hline
\end{tabular}




\begin{tabular}{|c|c|c|c|c|c|c|c|c|}
\hline Planned Staffing (Full & Time Equi & valent) & & Note: J & ob Family & Inly After & 1997 & \\
\hline Job Family & Number. & ४४। & अ०। & \$ै. & ४ै। & ४४। & ४४४ै & \%४। \\
\hline Job Category & & 1996 & 1997 & 1998 & 1999 & 2000 & .2001 & 2002 \\
\hline HVAC & & & & & & & & \\
\hline Machinists & & & & & & & & \\
\hline Masons & & & & & & & & \\
\hline Millwrights & & & & & & & & \\
\hline Painters & & & & & & & & \\
\hline Plmbrs/Pipefitters & & & & & & & & \\
\hline $\begin{array}{l}\text { Struct/Meta1 } \\
\text { Workers }\end{array}$ & & & & & & & & \\
\hline $\begin{array}{l}\text { Vehicl/Mobile } \\
\text { Equip. } \\
\text { Mechanics }\end{array}$ & & & & & & & & \\
\hline Welders & & & & & & & & \\
\hline other & & & & & & & & \\
\hline Subtotal & & & & & & & & \\
\hline OPEERATORS ? & \%०. \% & अ०० & ڤ. \% & ०ै। & ४ै। & $13 \%$ & ४ै২े & मे \\
\hline Lt. Vehicle Drivers & & & & & & & & \\
\hline $\begin{array}{l}\text { Material Moving } \\
\text { Equip. }\end{array}$ & & & & & & & & \\
\hline Nuclear Plant & & & & & & & & \\
\hline $\begin{array}{l}\text { Utilities Waste } \\
\text { Proces }\end{array}$ & & & & & & & & \\
\hline Other & & & & & & & & \\
\hline Subtotal & & & & & & & & \\
\hline
\end{tabular}




\begin{tabular}{|c|c|c|c|c|c|c|c|c|}
\hline \multicolumn{4}{|c|}{ Planned Staffing (Full Time Equivalent) } & \multicolumn{5}{|c|}{ Note: Job Family Only After 1997} \\
\hline Job Famfly & Number: & \%!। & «॥ & \& : & ४४४ & 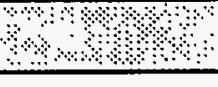 & ४४। & अ \\
\hline Job Category & & 1996 & 1997 & 1998 & 1999 & 2000 & 2001 & 2002 \\
\hline $\begin{array}{l}\text { ABOR } \& \text { GENERAL } \\
\text { WORKERS }\end{array}$ & & $\sqrt[8]{4}+$ & अै। & अे & भै & ? & अे। & +ै? \\
\hline \multicolumn{9}{|l|}{ Firefighters } \\
\hline \multicolumn{9}{|l|}{ Food Service } \\
\hline \multicolumn{9}{|l|}{ Hand/Help Lab Gen } \\
\hline \multicolumn{9}{|l|}{$\begin{array}{l}\text { Hand/Help Lab } \\
\text { Special }\end{array}$} \\
\hline \multicolumn{9}{|l|}{ Janitors/CTeaners } \\
\hline \multicolumn{9}{|l|}{ Laundry Workers } \\
\hline \multicolumn{9}{|l|}{ Security Guards } \\
\hline \multicolumn{9}{|l|}{ other } \\
\hline \multicolumn{9}{|l|}{ Subtotal } \\
\hline TOTAL FTES & & & & & & & & \\
\hline
\end{tabular}




\subsubsection{Engineering (WBS 6.5.4.4)}

Mission

The mission of the Engineering and Technical Support Services organization is to maintain the qualified and trained personnel to provide all required architectural, engineering, design, and drafting support to the Hanford site as required, either directiy or through outsourcing. The Manager of Engineering and Technical Support Services performs the role of the Chief Engineer of ICF KH and performs the duties required under the ICF KH license as a engineering corporation under Washington State Laws. The Manager of the VaTue Engineering and Architectural Services department performs the role of the Chief Architect of ICF KH and performs the duties required under the ICF $\mathrm{KH}$ license as an Architectural Corporation under Washington State Laws.

\section{Product/Services Provided}

The Engineering and Technical Support Services division provides the following services: Value Engineering and Architecture Services; Quality Inspection Services, Civil/Structural and Environmental Engineering Services; Data Automation Engineering Services; Control Systems Engineering Services; Mechanical Engineering Services; Design Services and Support which encompasses Application Design Services, As-Builting/Mapping/Surveying Services, Inner Area Design Services, Checking Services, and Design Support; Mechanisms Engineering Services, Electrical Engineering Services; Central Support Services which encompasses CAD/CAE Systems, Technical Documents and Administrative Support; and Systems and Industrial Engineering Services.

\section{Primary Customer}

The Primary customers of the Engineering and Technical Support Services Division are: ICF Kaiser Hanford, U.S. Department of Energy (RL), Westinghouse Hanford Company, and Pacific Northwest Laboratory. 


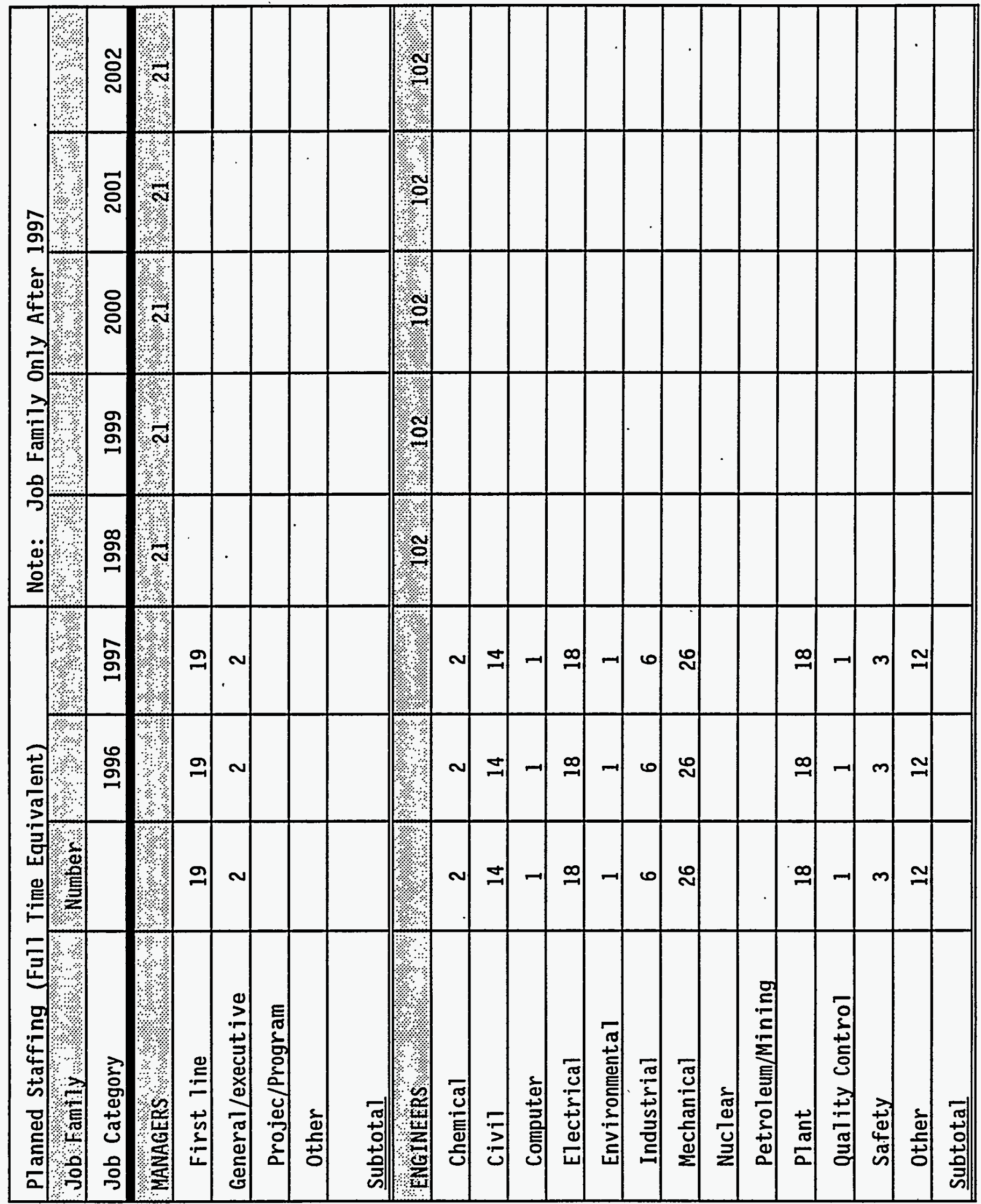




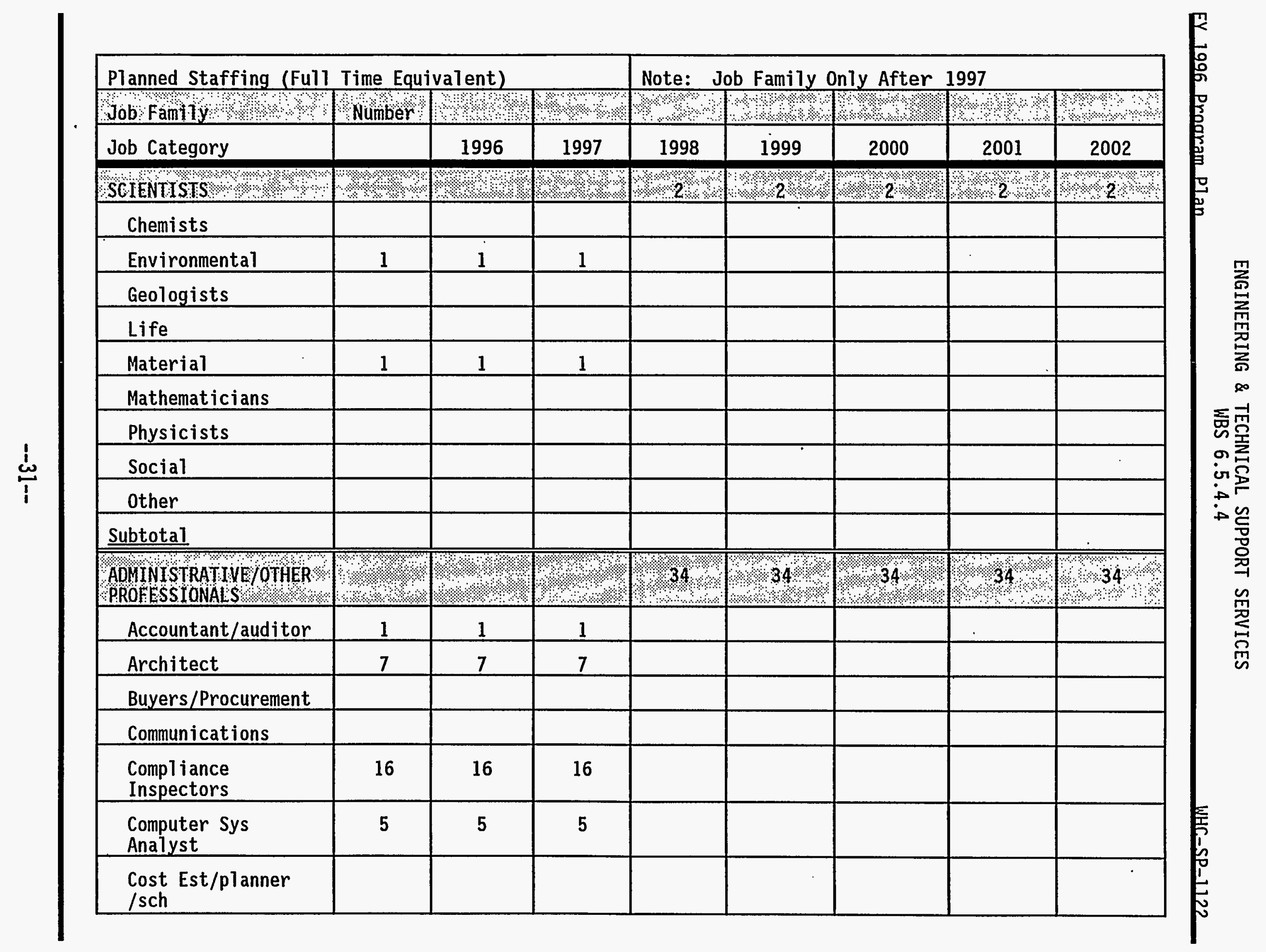




\begin{tabular}{|c|c|c|c|c|c|c|c|c|}
\hline \multicolumn{4}{|c|}{ Planned Staffing (Full Time Equivalent) } & \multicolumn{5}{|c|}{ Note: Job Family Only After 1997} \\
\hline Job Family & Number: & \%? & ४४४ & ९) & :४ै। & ४००.? & ४थे? & एे। \\
\hline Job Category & & 1996 & 1997 & 1998 & 1999 & 2000 & 2001 & 2002 \\
\hline \multicolumn{9}{|l|}{ Health Physics } \\
\hline \multicolumn{9}{|l|}{ Industrial Hygiene } \\
\hline \multicolumn{9}{|l|}{ Lawyers } \\
\hline $\begin{array}{l}\text { Personnel/1abor } \\
\text { Relations }\end{array}$ & 1 & 1 & 1 & & & & & \\
\hline \multicolumn{9}{|l|}{ Physicians } \\
\hline \multicolumn{9}{|l|}{$\begin{array}{l}\text { Physician } \\
\text { Assistant/Nurse }\end{array}$} \\
\hline \multicolumn{9}{|l|}{$\begin{array}{l}\text { Safeguard \& } \\
\text { Security }\end{array}$} \\
\hline $\begin{array}{l}\text { Tech Writers \& } \\
\text { Editors }\end{array}$ & $\dot{2}$ & 2 & 2 & & & & & \\
\hline Trainers & 1 & 1 & 1 & & & & & \\
\hline Other & 1 & 1 & 1 & & & & & \\
\hline \multicolumn{9}{|l|}{ Subtotal } \\
\hline $\begin{array}{l}\text { GENERAL } \\
\text { ADMIN/SECRETARY Y CLERK }\end{array}$ & \%? & \%? & 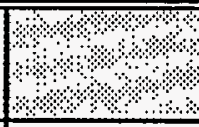 & \%? & $?_{25}$ & \% $25 \%$ & ? 25 & 25 \\
\hline Admin Assistant & 3 & 3 & 3 & & & & & \\
\hline Office Clerk (Gen) & 7 & 7 & 7 & & & & & \\
\hline \multicolumn{9}{|l|}{$\begin{array}{l}\text { Office Clerk } \\
\text { (Special) }\end{array}$} \\
\hline Secretaries & 15 & 15 & 15 & & & & & \\
\hline \multicolumn{9}{|l|}{$\begin{array}{l}\text { Typist/Word } \\
\text { Processor }\end{array}$} \\
\hline Other & & & & & & & & \\
\hline
\end{tabular}




\begin{tabular}{|c|c|c|c|c|c|c|c|c|}
\hline \multicolumn{4}{|c|}{ Planned Staffing (Ful1 Time Equivalent) } & \multicolumn{5}{|c|}{ Note: Job Family Only After 1997} \\
\hline Job Famfly & Nüber: & ४ै\% \% & ४४\% & ४४७? & ए०. & $1 \%$ & अ? & अ४४ \\
\hline Job Category & & 1996 & 1997 & 1998 & 1999 & 2000 & 2001 & 2002 \\
\hline \multicolumn{9}{|l|}{ Subtotal } \\
\hline 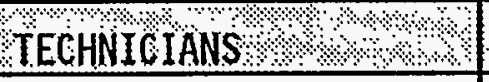 & 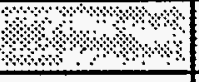 & ?.? & $1 \%$ & 3.53 & 2.53 & 8.43 .8 & ? $53 \%$ & $4,53 \%$ \\
\hline \multicolumn{9}{|l|}{$\begin{array}{l}\text { Computer } \\
\text { Operator/Coder }\end{array}$} \\
\hline Drafters & 52 & 52 & 52 & & & & & \\
\hline Engineers/Technicians & 1 & 1 & 1 & & & & & \\
\hline \multicolumn{9}{|l|}{$\begin{array}{l}\text { Environmental } \\
\text { Science } \\
\text { Technicians } \\
\end{array}$} \\
\hline \multicolumn{9}{|l|}{$\begin{array}{l}\text { Health Physics } \\
\text { Technicians }\end{array}$} \\
\hline \multicolumn{9}{|l|}{$\begin{array}{l}\text { Industrial } \\
\text { Saf/health } \\
\text { Technicians }\end{array}$} \\
\hline \multicolumn{9}{|l|}{$\begin{array}{l}\text { Instru/Control } \\
\text { Technicians }\end{array}$} \\
\hline \multicolumn{9}{|l|}{ Lab. Technicians } \\
\hline \multicolumn{9}{|l|}{ Media Technicians } \\
\hline \multicolumn{9}{|l|}{$\begin{array}{l}\text { Survey/Map } \\
\text { Technicians }\end{array}$} \\
\hline \multicolumn{9}{|l|}{ other } \\
\hline \multicolumn{9}{|l|}{ Subtotal } \\
\hline 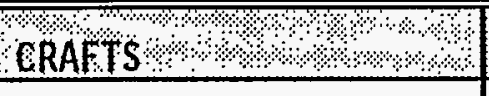 & ४ै। & 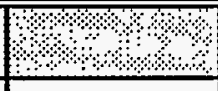 & ४ै\% & १ै२ै। & ४ै\% & $\% \%$ & १ै। & вथ \\
\hline Carpenters & & & & & & & & \\
\hline
\end{tabular}




\begin{tabular}{|c|c|c|c|c|c|c|c|c|}
\hline \multicolumn{4}{|c|}{ Planned Staffing (Full Time Equivalent) } & \multicolumn{5}{|c|}{ Note: Job Family Only After 1997} \\
\hline Job Family & Number: & $\%$ & $1 \%$ & ४४ & $4 \%$ & \%०२?! & \%थे। & ४४ \\
\hline Job Category & & 1996 & 1997 & 1998 & 1999 & 2000 & 2001 & 2002 \\
\hline Electricians & & & & & & & & \\
\hline HVAC & & & & & & & & \\
\hline Machinists & & & & & & & & \\
\hline Masons & & & & & & & & \\
\hline Millwrights & & & & & & & & \\
\hline Painters & & & & & & & & \\
\hline P1mbrs/Pipefitters & & & & & & & & \\
\hline $\begin{array}{l}\text { Struct/Metal } \\
\text { Workers }\end{array}$ & & & & & & & & \\
\hline $\begin{array}{l}\text { Vehicl/Mobile } \\
\text { Equip. } \\
\text { Mechanics }\end{array}$ & & & & & & & & \\
\hline Welders & & & & & & & & \\
\hline Other & & & & & & & & \\
\hline Subtotal & & & & & & & & \\
\hline OPERATORS : & अे\%?. & ४ै। । & ४ै०े & अै। & अै। & \%ै०० & ४० & 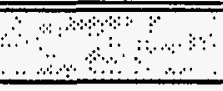 \\
\hline Lt. Vehicle Drivers & & & 。 & & & & & \\
\hline $\begin{array}{l}\text { Material Moving } \\
\text { Equip. }\end{array}$ & & & & & & & & \\
\hline Nuclear Plant & & & & & & & & \\
\hline $\begin{array}{l}\text { Utilities Waste } \\
\text { Proces }\end{array}$ & & & & & & & & \\
\hline Other & & & & & & & & \\
\hline Subtotal & & & & & & & & \\
\hline
\end{tabular}




\begin{tabular}{|c|c|c|c|c|c|c|c|c|}
\hline \multicolumn{4}{|c|}{ Planned Staffing (Full Time Equivalent) } & \multicolumn{5}{|c|}{ Note: Job Family Only After 1997} \\
\hline Job family & Number & ২২০ & 13 & \% & ४े। & अै। & +ै। & s. \\
\hline Job Category & & 1996 & 1997 & 1998 & 1999 & 2000 & 2001 & 2002 \\
\hline $\begin{array}{l}\text { LABOR \& GENERAL } \\
\text { WORKERS }\end{array}$ & & & ४ै? & ?ै? & ${ }^{2}+$ ? & ४ै। & मे? & में \\
\hline Firefighters & & & & - & & & & \\
\hline Food Service & & & & & & & & \\
\hline Hand/Help Lab Gen & & & & & & & & \\
\hline $\begin{array}{l}\text { Hand/Help Lab } \\
\text { Special }\end{array}$ & & & & & & & & \\
\hline Janitors/Cleaners & & & & & & & & \\
\hline Laundry Workers & & & & & & & & \\
\hline Security Guards & & & & & & & & \\
\hline other & & & & & & & & \\
\hline Subtotal & & & & & & & & \\
\hline TOTAL FTES & & & & & & & & \\
\hline
\end{tabular}


3.1.5 Tank Waste Remediation System (TWRS) Engineering \& Construction Services (WBS 6.5.4.5)

Mission

The TWRS Engineering and Construction Services Division, in it capacity as the functional project management entity, has the responsibility for planning, organizing, and managing the design and construction activities associated with Tank Farm Upgrades projects, Waste Retrieval projects, Tank Farm Restoration projects and Safe Operations projects.

\section{Products/Services Provided}

The TWRS Engineering and Construction Services Division provides the following: Project Management and Reporting, Title I, II and III Engineering, Value Engineering, Technical Evaluations, Design Services in the outer areas as well as in town, Project Controls including CPM schedules and Performance reports, Construction Management of fixed and unit price contracts, Management of outsourced engineering tasks, and Administrative Management.

\section{Primary Customers}

The primary customers of the TWRS Engineering and Construction Services Division are: ICF Kaiser Hanford, Westinghouse Hanford Company, and U.S. Department of Energy (RL). 


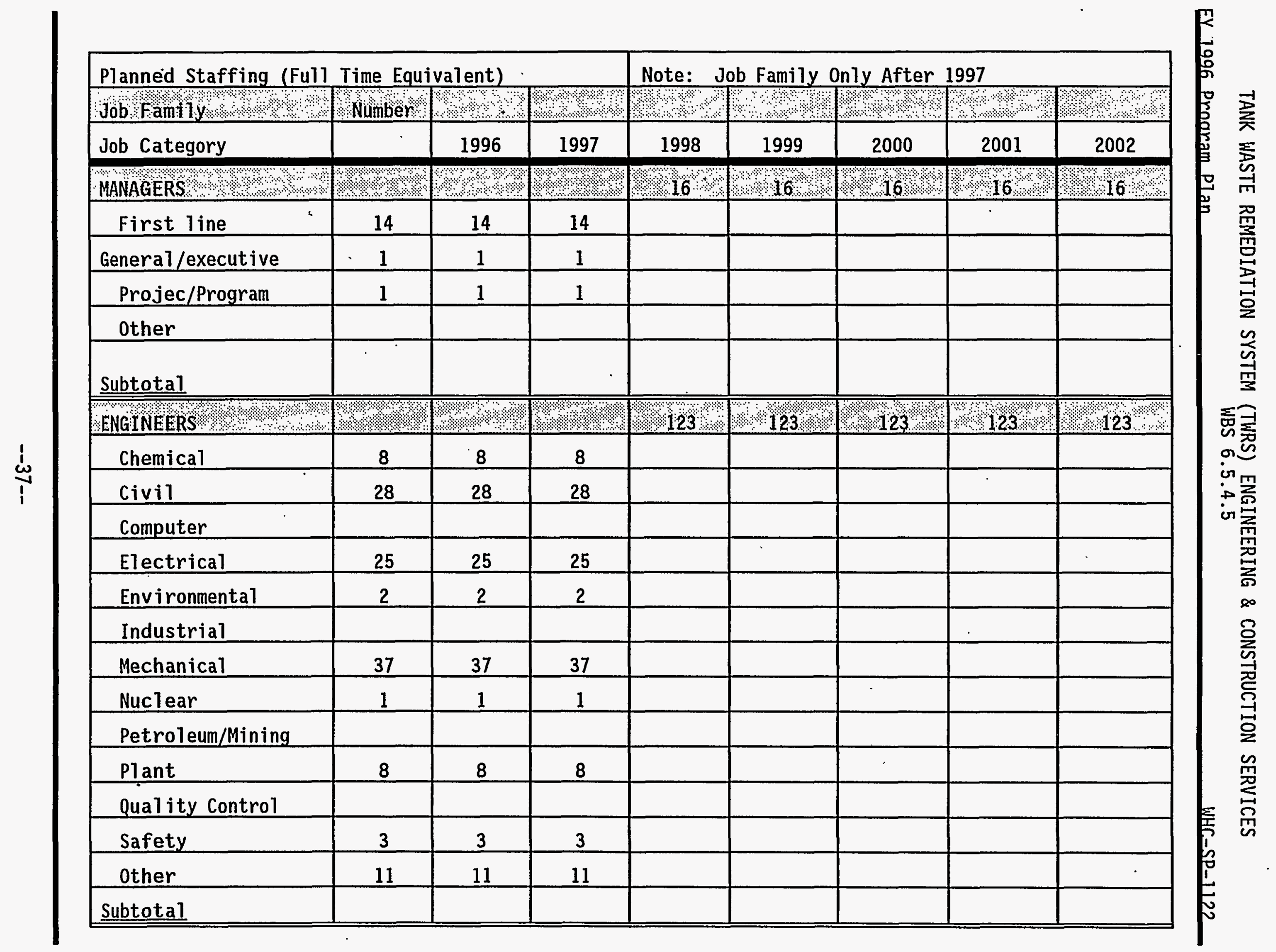




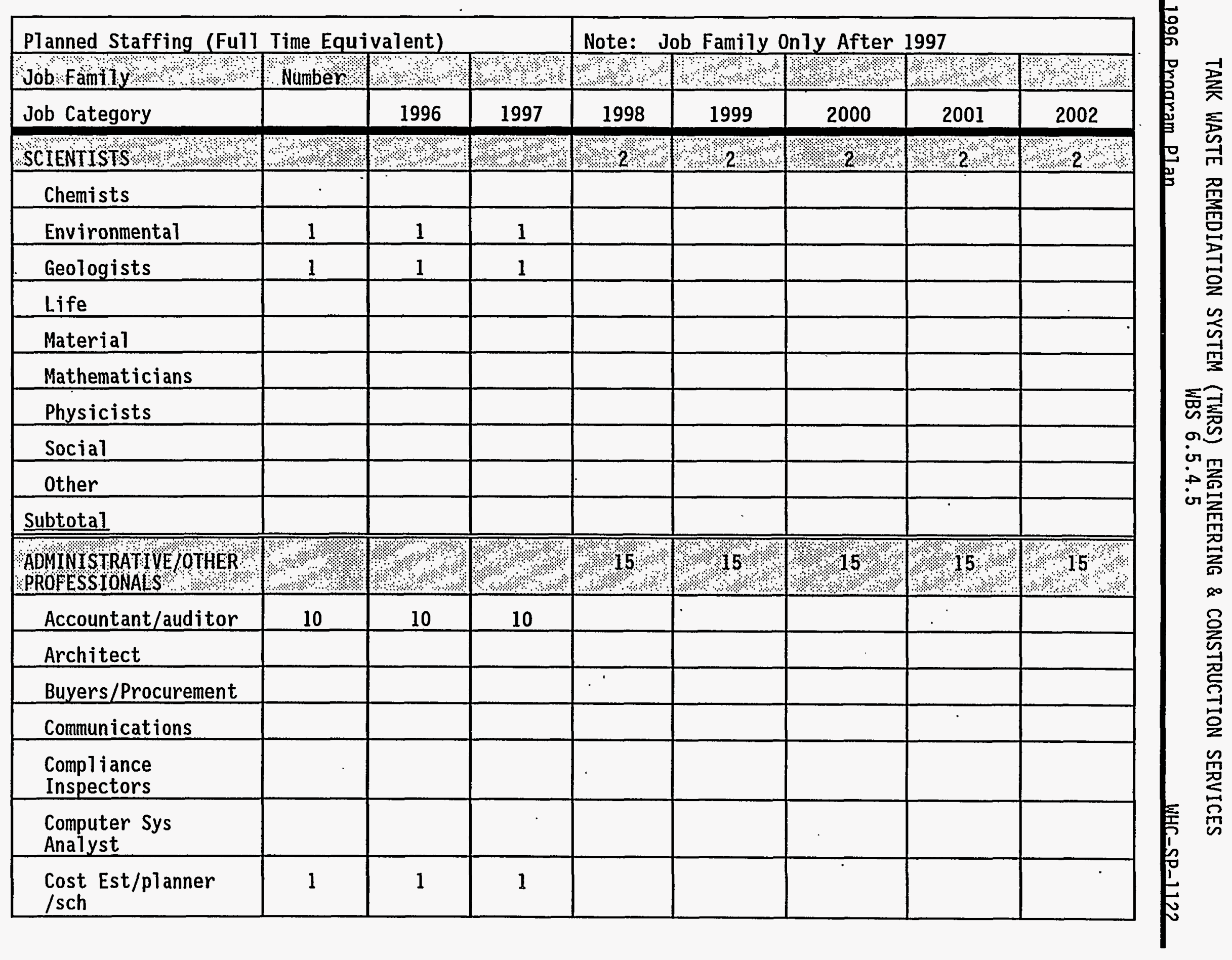




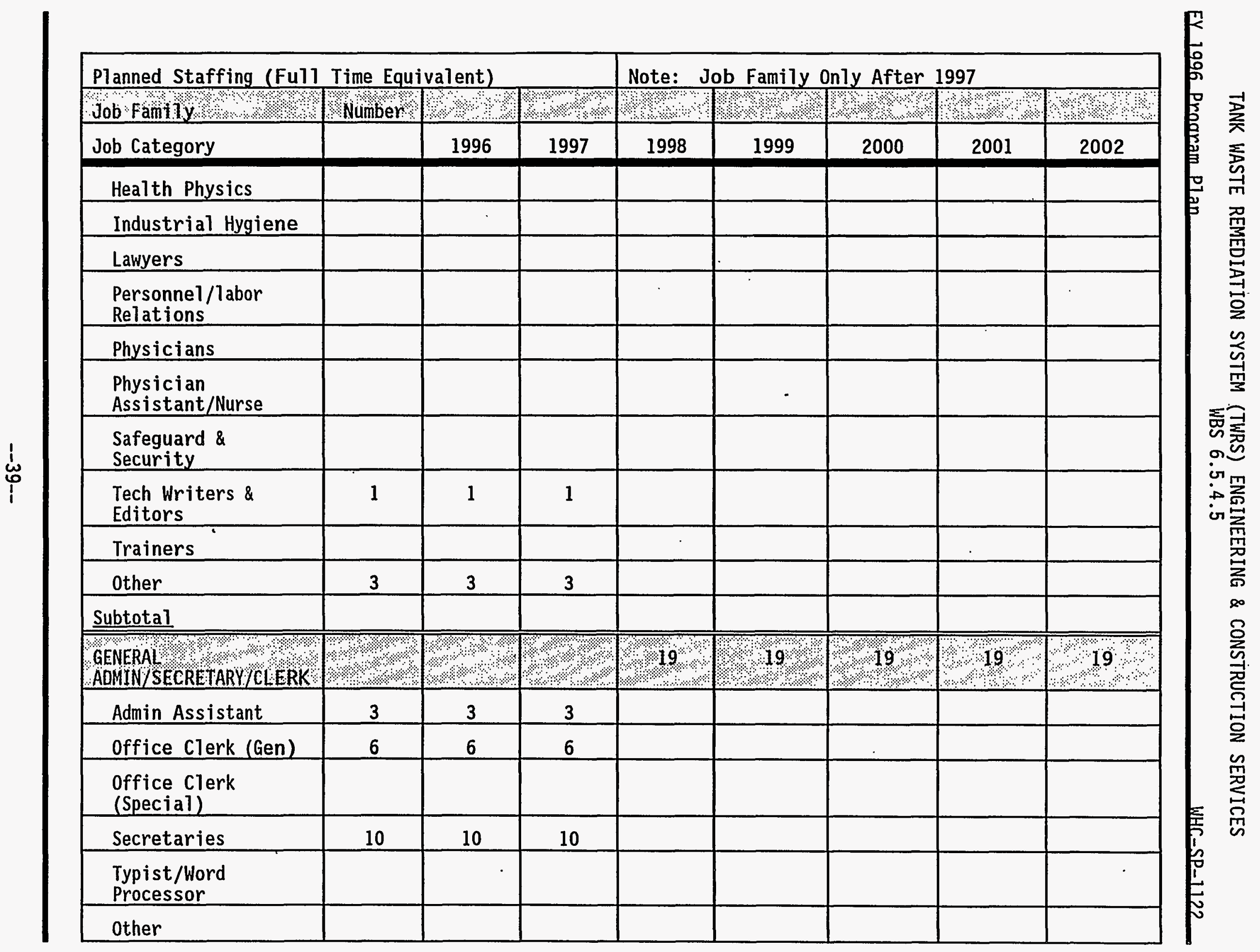




\begin{tabular}{|c|c|c|c|c|c|c|c|c|}
\hline Planned Staffing (Full & Time Equi & valent) & & Note: $\mathrm{J}$ & b Family $c$ & nly After & 1997 & \\
\hline Job famlly ${ }^{2}$ ४े। & Nümber & \%? & \%४ & ४ै. & ४ै? & ४\% ? & ४४ ४ & अै? \\
\hline Job Category & & $\cdot 1996$ & 1997 & 1998 & 1999 & 2000 & 2001 & 2002 \\
\hline Subtotal & & & & & & & & \\
\hline TECHNICIANS & \%४ै. & ४ঃ४? & $2.8 \%$ & 8,51 . & ?. 51 ? & ४ै. $51 \%$ & \% $51 \%$ & \\
\hline $\begin{array}{l}\text { Computer } \\
\text { Operator/Coder }\end{array}$ & & & & & & & & \\
\hline Drafters & 51 & 51 & 51 & & & & & \\
\hline Engineers/Technicians & & & & & & & & \\
\hline $\begin{array}{l}\text { Environmenta1 } \\
\text { Science } \\
\text { Technicians } \\
\end{array}$ & & & & & & & & \\
\hline $\begin{array}{l}\text { Health Physics } \\
\text { Technicians }\end{array}$ & & & & & & & & \\
\hline $\begin{array}{l}\text { Industrial } \\
\text { Saf/health } \\
\text { Technicians }\end{array}$ & & & & & & & & \\
\hline $\begin{array}{l}\text { Instru/Control } \\
\text { Technicians }\end{array}$ & & & & & & & & \\
\hline Lab. Technicians & & & & & & & & \\
\hline Media Technicians & & & & & & & & \\
\hline $\begin{array}{l}\text { Survey/Map } \\
\text { Technicians }\end{array}$ & & & & & & & & \\
\hline Other & & & & & & & & \\
\hline Subtotal & & & & & & & & \\
\hline CRAFTS & अ४े & ২ি & ४ै।.? & \%ै। & अै। & ४ै. & अै। & 8 \\
\hline Carpenters & & & & & & & & \\
\hline
\end{tabular}




\begin{tabular}{|c|c|c|c|c|c|c|c|c|}
\hline \multicolumn{4}{|c|}{ Planned Staffing (Ful1 Time Equivalent) } & \multicolumn{5}{|c|}{ Note: Job Family Only After 1997} \\
\hline Job faml $y$ y & Number: & \%० & ४२: & अैे & ४ै४४४ & $1 \%$ & ४ै। & ४ै\% \\
\hline Job Category & & 1996 & 1997 & 1998 & 1999 & 2000 & 2001 & 2002 \\
\hline \multicolumn{9}{|l|}{ Electricians } \\
\hline \multicolumn{9}{|l|}{ HVAC } \\
\hline \multicolumn{9}{|l|}{ Machinists } \\
\hline \multicolumn{9}{|l|}{ Masons } \\
\hline \multicolumn{9}{|l|}{ Millwrights } \\
\hline \multicolumn{9}{|l|}{ Painters } \\
\hline \multicolumn{9}{|l|}{ Plmbrs/Pipefitters } \\
\hline \multicolumn{9}{|l|}{$\begin{array}{l}\text { Struct/Metal } \\
\text { Workers }\end{array}$} \\
\hline \multicolumn{9}{|l|}{$\begin{array}{l}\text { Vehicl/Mobile } \\
\text { Equip. } \\
\text { Mechanics }\end{array}$} \\
\hline \multicolumn{9}{|l|}{ Welders } \\
\hline \multicolumn{9}{|l|}{ other } \\
\hline \multicolumn{9}{|l|}{ Subtotal } \\
\hline OPERATORS & ?०? & \%) & ॠथ & \%ै\% & \%े \% & \%? & ४०० & ? \\
\hline \multicolumn{9}{|l|}{ Lt. Vehicle Drivers } \\
\hline \multicolumn{9}{|l|}{$\begin{array}{l}\text { Material Moving } \\
\text { Equip. }\end{array}$} \\
\hline \multicolumn{9}{|l|}{ Nuclear Plant } \\
\hline \multicolumn{9}{|l|}{$\begin{array}{l}\text { Utilities Waste } \\
\text { Proces }\end{array}$} \\
\hline \multicolumn{9}{|l|}{ other } \\
\hline Subtotal & & & & & & & & \\
\hline
\end{tabular}




\begin{tabular}{|c|c|c|c|c|c|c|c|c|}
\hline \multicolumn{4}{|c|}{ Planned Staffing (Full Time Equivalent) } & \multicolumn{5}{|c|}{ Note: Job Family Only After 1997} \\
\hline Job Fam $1 \mathrm{y}$ \% 3 & Namber: & ४ै। & \%॰ & 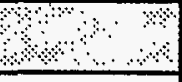 & +? & 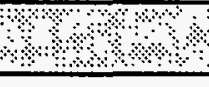 & ४ै। & ४०े \\
\hline Job Category & & 1996 & 1997 & 1998 & 1999 & 2000 & 2001 & 2002 \\
\hline $\begin{array}{l}\text { LABOR \& GENERAL } \\
\text { WORKERS }\end{array}$ & +ै? & अै? & $4 \%$ & भै & 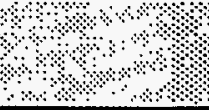 & $r_{4}+4$ & भै? & +ै: \\
\hline Firefighters & & & & & & & & \\
\hline Food Service & & & & & & & & \\
\hline Hand/Help Lab Gen & & & & & & & & \\
\hline $\begin{array}{l}\text { Hand/Help Lab } \\
\text { Special }\end{array}$ & & & & & & & & \\
\hline Janitors/Cleaners & & & & & & & & \\
\hline Laundry Workers & & & & & & & & \\
\hline Security Guards & & & & & & & & \\
\hline Other & & & & & & & & \\
\hline Subtotal & & & & & & & & \\
\hline TOTAL FTES & & & & & & & & \\
\hline
\end{tabular}




\subsubsection{Spent Nuclear Fuels (SNF) (WBS 6.5.4.6)}

\section{Mission}

The SNF Project and Path Forward Projects Division, in it capacity as the functional project management entity, has the responsibility for planning, organizing, and managing the design and construction activities associated with the K-Basin Essential Systems Recovery Project, the Hanford Spent Nuclear Fuel Staging and Storage Facility Project, and the Spent Nuclear Fuel Stabilization Facility Project.

\section{Product/Services Provided}

The SNF Division provides the following: Project Management, Engineering, Design Services, Project Controls, Construction Management, and Administration Management.

\section{Primary Customers}

The primary customers of the SNF Division are: ICF Kaiser Hanford, Westinghouse Hanford Company, and U.S. Department of Energy (RL). 


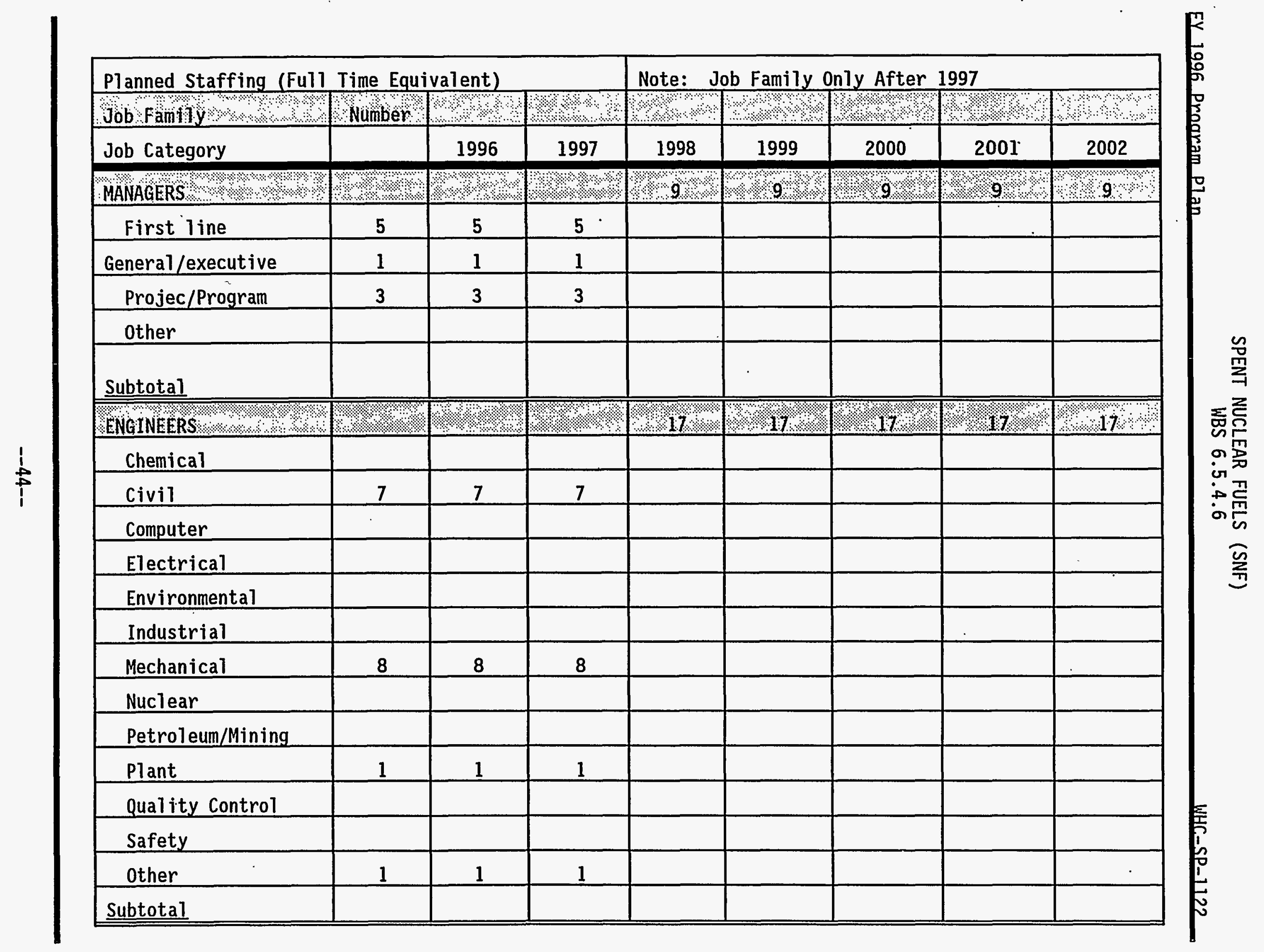




\begin{tabular}{|c|c|c|c|c|c|c|c|c|}
\hline \multicolumn{4}{|c|}{ Planned Staffing (Full Time Equivalent) } & \multicolumn{5}{|c|}{ Note: Job Family Only After 1997} \\
\hline Job family & Numben & ४ै? & ९ै\% & २े०० & ४४ ४ & ४ै४े? & ४ै। & मे. \\
\hline Job Category & & 1996 & 1997 & 1998 & 1999 & 2000 & 2001 & 2002 \\
\hline SCIENTISTS & ४ ২ ২ & ४०। & 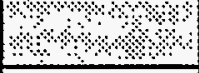 & ४े & ४४४४९: & 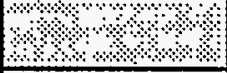 & ४ै०े। & ४ै। \\
\hline \multicolumn{9}{|l|}{ Chemists } \\
\hline \multicolumn{9}{|l|}{ Environmental } \\
\hline \multicolumn{9}{|l|}{ Geologists } \\
\hline \multicolumn{9}{|l|}{ Life } \\
\hline \multicolumn{9}{|l|}{ Material } \\
\hline \multicolumn{9}{|l|}{ Mathematicians } \\
\hline \multicolumn{9}{|l|}{ Physicists } \\
\hline \multicolumn{9}{|l|}{ Social } \\
\hline \multicolumn{9}{|l|}{ other } \\
\hline \multicolumn{9}{|l|}{ Subtotal } \\
\hline $\begin{array}{l}\text { ADMINISTRATIVE } / 0 \text { THER } \\
\text { PROFESSIONALS }\end{array}$ & $4 \% ?$ & ॰ \%? ? & थै? ? & \%? & $\%$ & $8 \% ? \%$ & ?े? & \% 5 \\
\hline Accountant/auditor & 2 & 2 & 2 & & & & & \\
\hline \multicolumn{9}{|l|}{ Architect } \\
\hline \multicolumn{9}{|l|}{ Buyers/Procurement } \\
\hline \multicolumn{9}{|l|}{ Communications } \\
\hline \multicolumn{9}{|l|}{$\begin{array}{l}\text { Compliance } \\
\text { Inspectors }\end{array}$} \\
\hline \multicolumn{9}{|l|}{$\begin{array}{l}\text { Computer Sys } \\
\text { Analyst }\end{array}$} \\
\hline $\begin{array}{l}\text { Cost Est/planner } \\
\text { /sch }\end{array}$ & & & & & & & & \\
\hline
\end{tabular}




\begin{tabular}{|c|c|c|c|c|c|c|c|c|}
\hline \multicolumn{9}{|c|}{\begin{tabular}{l|l} 
Planned Staffing (Full Time Equivalent) & Note: Job Family Only After 1997
\end{tabular}} \\
\hline Job Famfly & Number: & 28 & ४४०\%? & $\%$ & ४ै\% & $1 \% \odot$ & ४४४४। & \\
\hline Job Category & & 1996 & 1997 & 1998 & 1999 & 2000 & 2001 & 2002 \\
\hline \multicolumn{9}{|l|}{ Health Physics } \\
\hline \multicolumn{9}{|l|}{ Industrial Hygiene } \\
\hline \multicolumn{9}{|l|}{ Lawyers } \\
\hline \multicolumn{9}{|l|}{$\begin{array}{l}\text { Personnel/1abor } \\
\text { Relations }\end{array}$} \\
\hline \multicolumn{9}{|l|}{ Physicians } \\
\hline \multicolumn{9}{|l|}{$\begin{array}{l}\text { Physician } \\
\text { Assistant/Nurse }\end{array}$} \\
\hline \multicolumn{9}{|l|}{$\begin{array}{l}\text { Safeguard \& } \\
\text { Security }\end{array}$} \\
\hline \multicolumn{9}{|l|}{$\begin{array}{l}\text { Tech Writers \& } \\
\text { Editors }\end{array}$} \\
\hline \multicolumn{9}{|l|}{ Trainers } \\
\hline other & 3 & 3 & 3 & & & & & \\
\hline \multicolumn{9}{|l|}{ Subtotal } \\
\hline $\begin{array}{l}\text { GENERAL, } \\
\text { ADMIN/SECRETARY /CLERK }\end{array}$ & \%ै। & \%? & \%? \% \% & ४ै: & अ?\%? & \%? & मै? & 7 \\
\hline Admin Assistant & 1 & 1 & 1 & & & & & \\
\hline Office Clerk (Gen) & 4 & 4 & 4 & & & & & \\
\hline \multicolumn{9}{|l|}{$\begin{array}{l}\text { Office Clerk } \\
\text { (Special) }\end{array}$} \\
\hline Secretaries & 2 & 2 & 2 & & & & & \\
\hline \multicolumn{9}{|l|}{$\begin{array}{l}\text { Typist/Word } \\
\text { Processor }\end{array}$} \\
\hline other & & & & & & & & \\
\hline
\end{tabular}




\begin{tabular}{|c|c|c|c|c|c|c|c|c|}
\hline \multicolumn{4}{|c|}{ Planned Staffing (Full Time Equivalent) } & \multicolumn{5}{|c|}{ Note: Job Family Only After 1997} \\
\hline Job Family & Number: & $1 . \%$ & 3 & ४*\% & \%? & 3..?: & ४०४ & \%ै? \\
\hline Job Category & 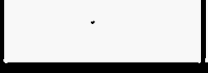 & 1996 & 1997 & 1998 & 1999 & 2000 & 2001 & 2002 \\
\hline \multicolumn{9}{|l|}{ Subtotal } \\
\hline TECHNICIANS & ४े ४े? & ४ै०े. & $2 \%$ & $6 \%$ & \%ै, 6 ?: & 36, & मे 6 & अ.? \\
\hline \multicolumn{9}{|l|}{$\begin{array}{l}\text { Computer } \\
\text { Operator/Coder }\end{array}$} \\
\hline Drafters & 6 & 6 & 6 & & & & & \\
\hline \multicolumn{9}{|l|}{ Engineers/Technicians } \\
\hline \multicolumn{9}{|l|}{$\begin{array}{l}\text { Environmental } \\
\text { Science } \\
\text { Technicians }\end{array}$} \\
\hline \multicolumn{9}{|l|}{$\begin{array}{l}\text { Health Physics } \\
\text { Technicians }\end{array}$} \\
\hline \multicolumn{9}{|l|}{$\begin{array}{l}\text { Industrial } \\
\text { Saf/health } \\
\text { Technicians }\end{array}$} \\
\hline \multicolumn{9}{|l|}{$\begin{array}{l}\text { Instru/Control } \\
\text { Technicians }\end{array}$} \\
\hline \multicolumn{9}{|l|}{ Lab. Technicians } \\
\hline \multicolumn{9}{|l|}{ Media Technicians } \\
\hline \multicolumn{9}{|l|}{$\begin{array}{l}\text { Survey/Map } \\
\text { Technicians }\end{array}$} \\
\hline \multicolumn{9}{|l|}{ Other } \\
\hline \multicolumn{9}{|l|}{ Subtotal } \\
\hline GRAFTS $\%$ ८ & ४४४े & ४ै\%? & २े।? & \%? & ॥ै. & ४४ ४ै। & \%०े & मिख \\
\hline Carpenters & & & & & & & & \\
\hline
\end{tabular}




\begin{tabular}{|c|c|c|c|c|c|c|c|c|}
\hline \multicolumn{4}{|c|}{ Planned Staffing (Full Time Equivalent) } & \multicolumn{5}{|c|}{ Note: Job Family Oniy After 1997} \\
\hline Job Family & Númbers & ४ै। & ४४४ & ४ै। ४। & ४४४४। & 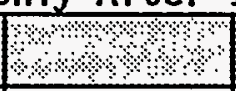 & ४ै. & अथे \\
\hline Job Category & & 1996 & 1997 & 1998 & 1999 & 2000 & 2001 & 2002 \\
\hline \multicolumn{9}{|l|}{ Electricians } \\
\hline \multicolumn{9}{|l|}{ HVAC } \\
\hline \multicolumn{9}{|l|}{ Machinists } \\
\hline \multicolumn{9}{|l|}{ Masons } \\
\hline \multicolumn{9}{|l|}{ Millwrights } \\
\hline \multicolumn{9}{|l|}{ Painters } \\
\hline \multicolumn{9}{|l|}{ Plmbrs/Pipefitters } \\
\hline \multicolumn{9}{|l|}{$\begin{array}{l}\text { Struct/Metal } \\
\text { Workers }\end{array}$} \\
\hline \multicolumn{9}{|l|}{$\begin{array}{l}\text { Vehicl/Mobile } \\
\text { Equip. } \\
\text { Mechanics }\end{array}$} \\
\hline \multicolumn{9}{|l|}{ Welders } \\
\hline \multicolumn{9}{|l|}{ Other } \\
\hline \multicolumn{9}{|l|}{ Subtotal } \\
\hline OPERATORS & ४ै\%? & $\% \% \%$ & २े२? & \%ै\% & ४ै। & ४ै। & ४ै। & \%" \\
\hline \multicolumn{9}{|l|}{ Lt. Vehicle Drivers } \\
\hline \multicolumn{9}{|l|}{$\begin{array}{l}\text { Material Moving } \\
\text { Equip. }\end{array}$} \\
\hline \multicolumn{9}{|l|}{ Nuclear Plant } \\
\hline \multicolumn{9}{|l|}{$\begin{array}{l}\text { Utilities Waste } \\
\text { Proces }\end{array}$} \\
\hline \multicolumn{9}{|l|}{ other } \\
\hline Subtotal & & & & & & & & \\
\hline
\end{tabular}




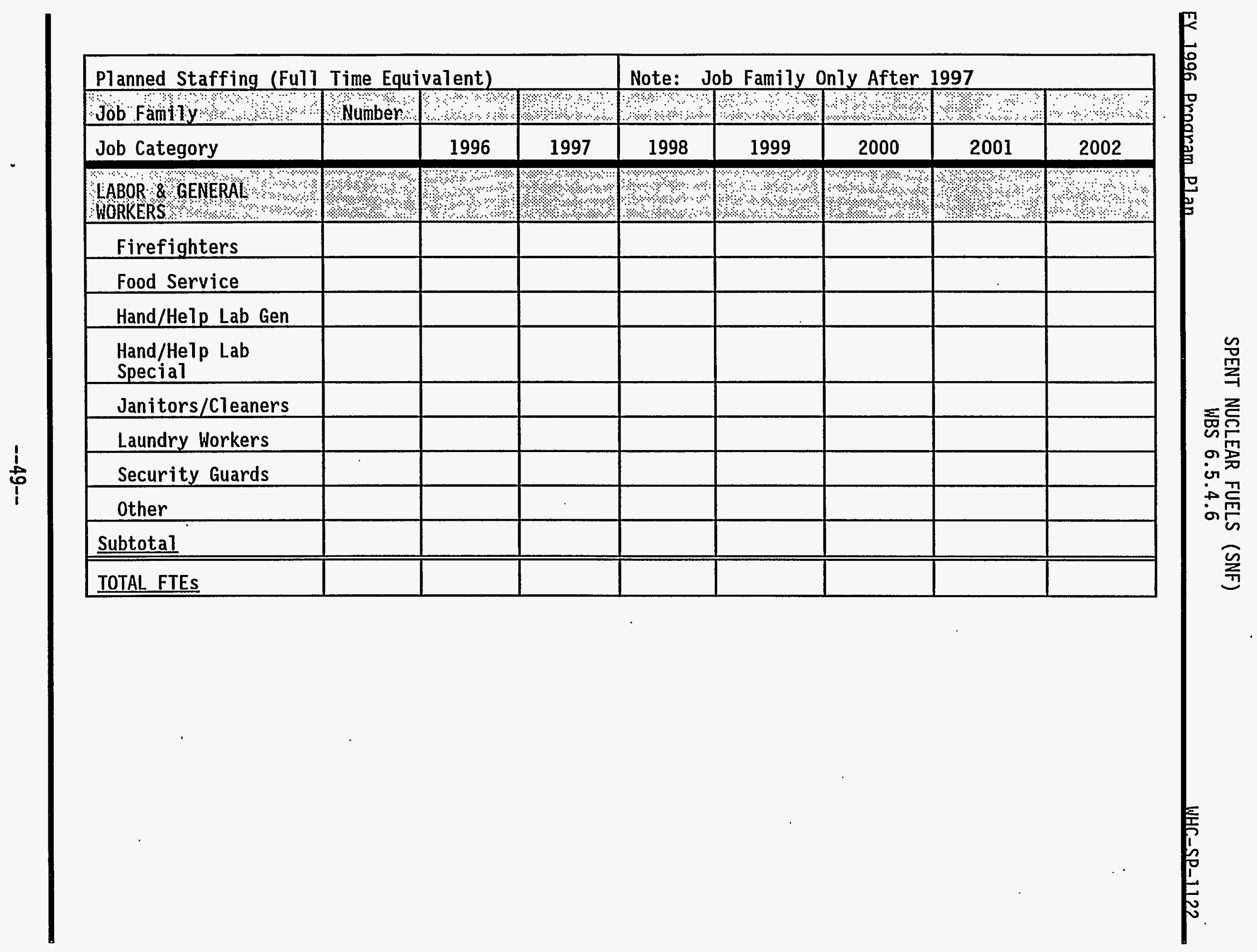


3.1.7 PNL Landlord, Engineering and Construction Management (WBS 6.5.4.7)

\section{Mission}

The PNL Landiord, Engineering and Construction Management Division, in its capacity as the functional project management entity, has the responsibility for planning, organizing, and managing the design and construction activities associated with the Pacific Northwest Laboratory Project, Facilities and Utilities Projects, EMSL and support of Construction Forces and ES\&H activities associated with the Division.

\section{Products/Services Provided}

The PNL Landlord, Engineering and Construction Management Division provides the following: Project Management, Engineering, Design Services, Project Controls, Construction Management, and Administration Management.

\section{Primary Customer}

The primary customers of the PNL Landlord, Engineering and Construction Management Division are: ICF Kaiser Hanford Westinghouse Hanford Company, Pacific Northwest Laboratory, and U.S. Department of Energy (RL). 


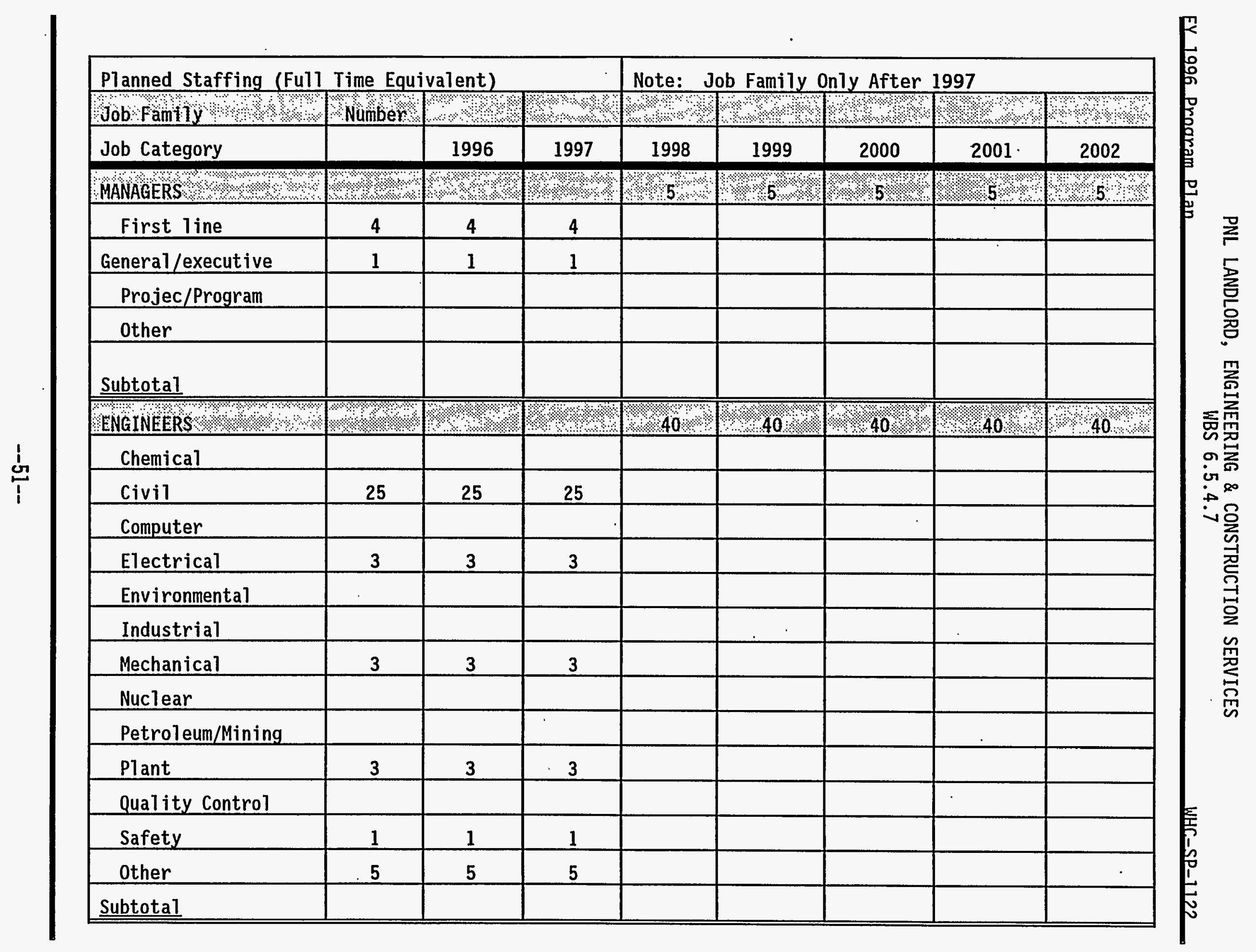




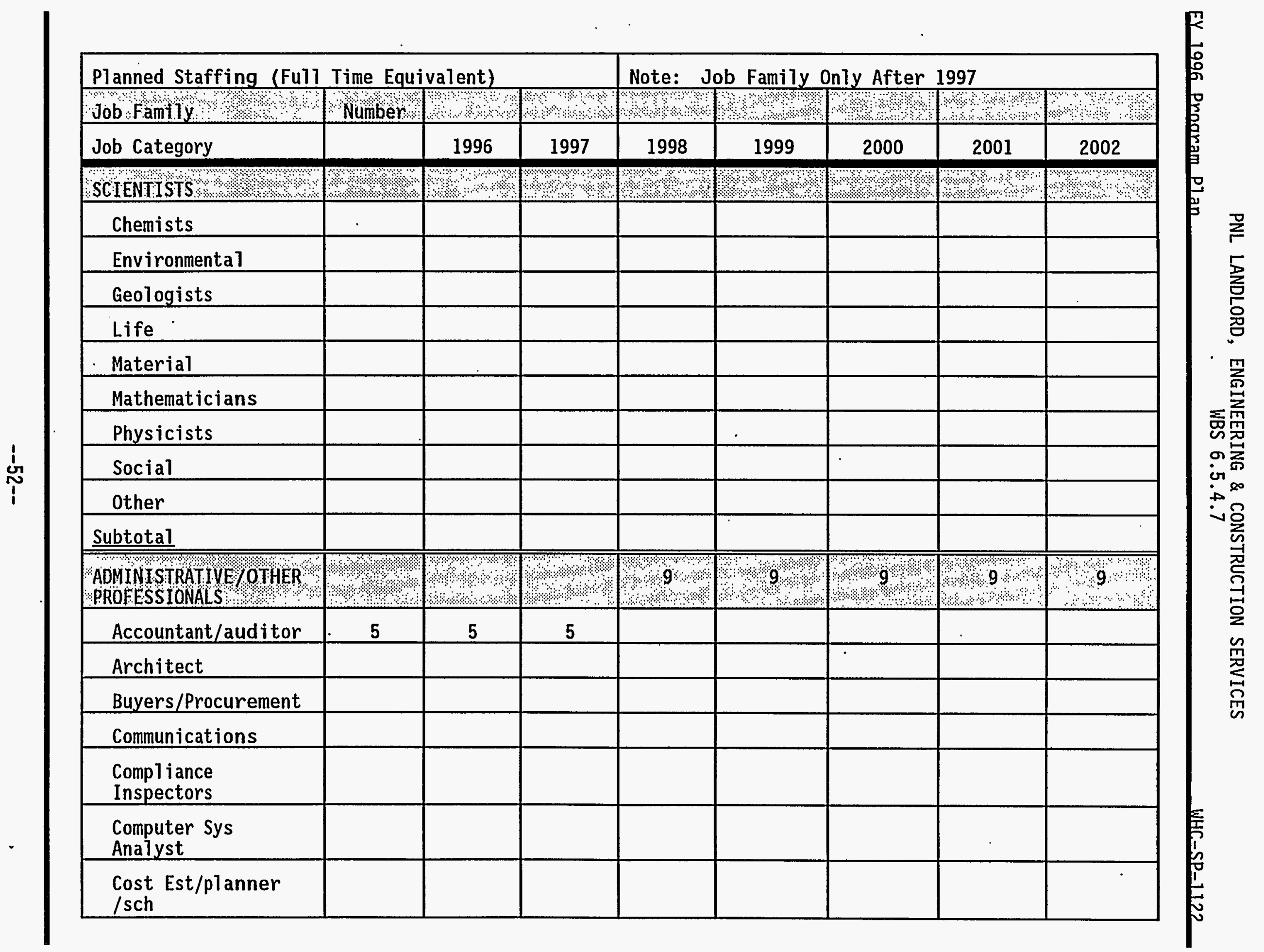




\begin{tabular}{|c|c|c|c|c|c|c|c|c|}
\hline \multicolumn{4}{|c|}{ Planned Staffing (Ful1 Time Equivalent) } & \multicolumn{5}{|c|}{ Note: Job Family Only After 1997} \\
\hline Job Famlly & Number: & \%) & ४\% & \%?: & ४थ\% & ঋ\% \% & \%०४ & ४ि \\
\hline Job Category & & 1996 & 1997 & 1998 & 199.9 & 2000 & 2001 & 2002 \\
\hline Health Physics & & & & & & & & \\
\hline Industrial Hygiene & & & & & & & . & \\
\hline Lawyers & & & & & & & & \\
\hline $\begin{array}{l}\text { Personne1//abor } \\
\text { Relations }\end{array}$ & & & & & & & & \\
\hline Physicians & & & & & & & & \\
\hline $\begin{array}{l}\text { Physician } \\
\text { Assistant/Nurse }\end{array}$ & & & & & & & & \\
\hline $\begin{array}{l}\text { Safeguard \& } \\
\text { Security }\end{array}$ & & & & & & & & \\
\hline $\begin{array}{l}\text { Tech Writers \& } \\
\text { Editors }\end{array}$ & & & & & & & & \\
\hline Trainers & & & . & & & & & \\
\hline Other & 4 & 4 & 4 & & & & & \\
\hline Subtotal & & & & & & & & \\
\hline $\begin{array}{l}\text { GENERAL, } \\
\text { AQMIN/SECRETARY/CLERK }\end{array}$ & + & \%? & \%?:? & ? $7 \%$ & अ? & \% & $1 \%$ & 27 \\
\hline Admin Assistant & & & & & & & & \\
\hline Office Clerk (Gen) & 5 & 5 & 5 & & & & & \\
\hline $\begin{array}{l}\text { Office Clerk } \\
\text { (Special) }\end{array}$ & & & & & & & & \\
\hline Secretaries & 2 & 2 & 2 & & & & & \\
\hline $\begin{array}{l}\text { Typist/Word } \\
\text { Processor }\end{array}$ & & & & & & & & \\
\hline Other & & & & & & & & \\
\hline
\end{tabular}




\begin{tabular}{|c|c|c|c|c|c|c|c|c|}
\hline \multicolumn{4}{|c|}{ Planned Staffing (Full Time Equivalent) } & \multicolumn{5}{|c|}{ Note: Job Family Only After 1997} \\
\hline Job Family & Number: & बो & २२। & ?. & । & ४ै? & अै। & ४ि। \\
\hline Job Category & & 1996 & 1997 & 1998. & 1999 & 2000 & 2001 & 2002 \\
\hline Subtotal & & & & & & & & \\
\hline TECHNICIANS & ४४४४ & \%० & \%०? & ४२ & 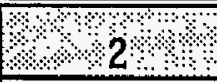 & $32_{2}$ & \%ै? & ? 2 \\
\hline $\begin{array}{l}\text { Computer } \\
\text { Operator/Coder }\end{array}$ & & & & & & & & \\
\hline Drafters & 2 & 2 & 2 & & & & & \\
\hline Engineers/Technicians & & & & & & & & \\
\hline $\begin{array}{l}\text { Environmental } \\
\text { Science } \\
\text { Technicians }\end{array}$ & & & & & & & & \\
\hline $\begin{array}{l}\text { Health Physics } \\
\text { Technicians }\end{array}$ & & & & & & & & \\
\hline $\begin{array}{l}\text { Industrial } \\
\text { Saf/health } \\
\text { Technicians }\end{array}$ & & & & & & & & \\
\hline $\begin{array}{l}\text { Instru/Control } \\
\text { Technicians }\end{array}$ & & & & & & & & \\
\hline Lab. Technicians & & & & & & & & \\
\hline Media Technicians & & & & & & & & \\
\hline $\begin{array}{l}\text { Survey/Map } \\
\text { Technicians }\end{array}$ & & & & & & & & \\
\hline other & & & & & & & & \\
\hline Subtotal & & & & & & & & \\
\hline CRAFTS $\%$ & \%॰ & ४४ & \%थ & थे? & ४ै। & \%े & क्ष & 2क \\
\hline Carpenters & & & & & & & & \\
\hline
\end{tabular}




\begin{tabular}{|c|c|c|c|c|c|c|c|c|}
\hline \multicolumn{4}{|c|}{ Planned Staffing (Full Time Equivalent) } & \multicolumn{5}{|c|}{ Note: Job Family Only After 1997} \\
\hline Job family & Nümber & ४४ & ?े: & ४े & 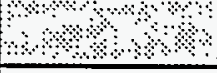 & ४ै? & ४ै४े & ॥. \\
\hline Job Category & & 1996 & 1997 & 1998 & 1999 & 2000 & 2001 & 2002 \\
\hline \multicolumn{9}{|l|}{ Electricians } \\
\hline \multicolumn{9}{|l|}{ HVAC } \\
\hline \multicolumn{9}{|l|}{ Machinists } \\
\hline \multicolumn{9}{|l|}{ Masons } \\
\hline \multicolumn{9}{|l|}{ Millwrights } \\
\hline \multicolumn{9}{|l|}{ Painters } \\
\hline \multicolumn{9}{|l|}{ Plmbrs/Pipefitters } \\
\hline \multicolumn{9}{|l|}{$\begin{array}{l}\text { Struct/Metal } \\
\text { Workers }\end{array}$} \\
\hline \multicolumn{9}{|l|}{$\begin{array}{l}\text { Vehicl/Mobile } \\
\text { Equip. } \\
\text { Mechanics }\end{array}$} \\
\hline \multicolumn{9}{|l|}{ Welders } \\
\hline \multicolumn{9}{|l|}{ Other } \\
\hline \multicolumn{9}{|l|}{ Subtotal } \\
\hline OPERATORS & अै? & अै। & \%॰ & ४ै० & अ\% \% & ४ै। & अे० & अ० \\
\hline \multicolumn{9}{|l|}{ Lt. Vehicle Drivers } \\
\hline \multicolumn{9}{|l|}{$\begin{array}{l}\text { Material Moving } \\
\text { Equip. }\end{array}$} \\
\hline \multicolumn{9}{|l|}{ Nuclear Plant } \\
\hline \multicolumn{9}{|l|}{$\begin{array}{l}\text { Utilities Waste } \\
\text { Proces }\end{array}$} \\
\hline \multicolumn{9}{|l|}{ Other } \\
\hline Subtotal & & & & & & & & \\
\hline
\end{tabular}




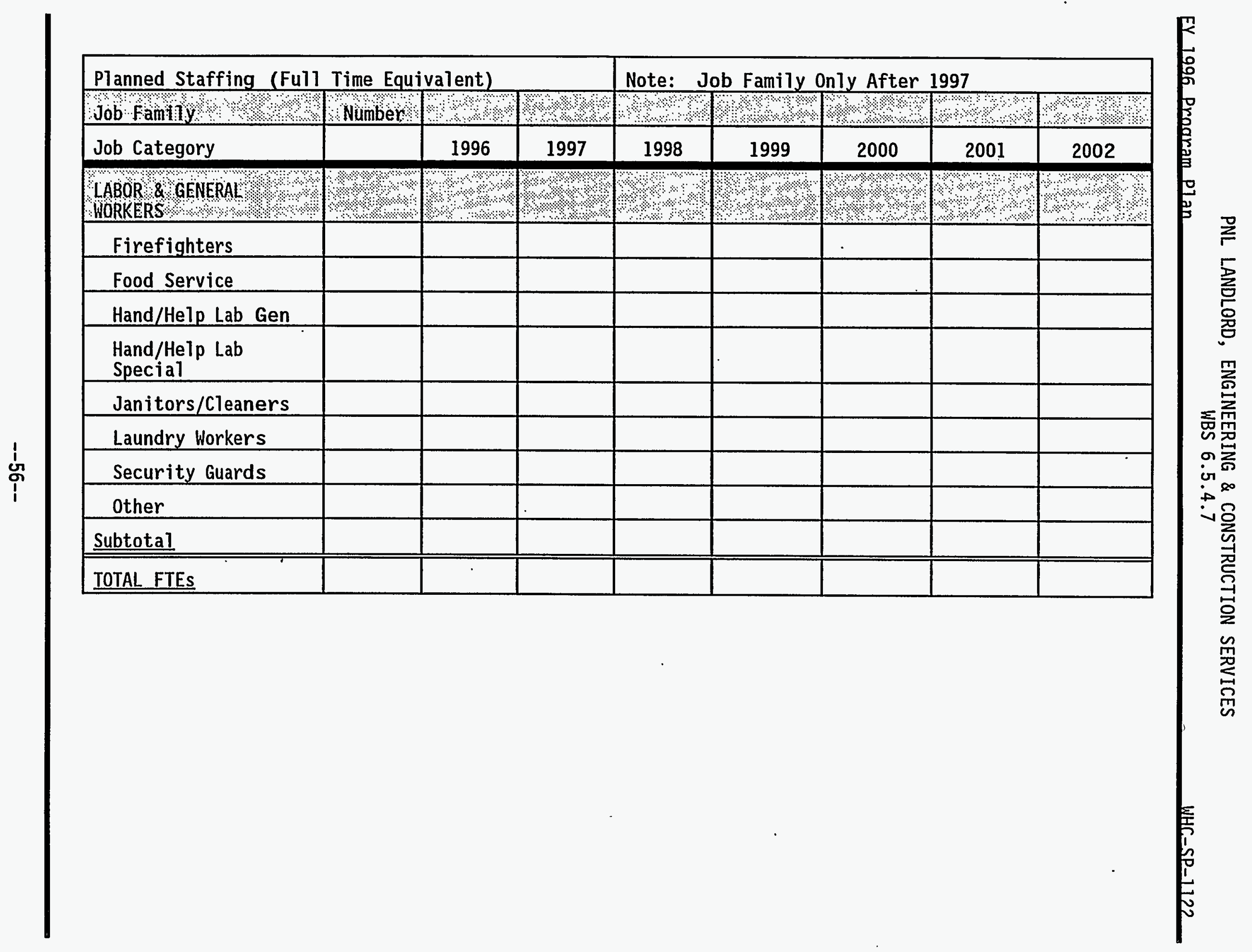


3.1.8 Transition Projects/Site Engineering \& Construction Services (WBS 6.5.4.8)

Mission

The Transition Projects/Site Engineering \& Construction Services Division, in its capacity as the functional Engineering and Construction management entity, has the responsibility for planning, organizing, and managing the construction and design activities associated with Environmental Restoration, Facility Transition, Hanford Environmental Compliance and. Solid Waste.

\section{Products/Services Provided}

The Transition Project and Site Engineering \& Construction Services Division provides the following: Project Management, Engineering, Design Services, Project Controls, Construction Management, and Administration Management.

\section{Primary Customers}

The primary customers of the Transition and Site Engineering \& Construction Services Division are: ICF Kaiser Hanford, Westinghouse Hanford, and U.S. Department of Energy (RL). 


\begin{tabular}{|c|c|c|c|c|c|c|c|c|}
\hline \multicolumn{4}{|c|}{ Planned Staffing (Full Time Equivalent) } & \multicolumn{5}{|c|}{ Note: Job Family Only After 1997} \\
\hline Job Famlly & Number & मे? & $1 \%$ & \%। & \%? & ४४४े & ৫४४ঃ & \%०। \\
\hline Job Category & & 1996 & 1997 & 1998 & 1999 & 2000 & 2001 & 2002 \\
\hline MANAGERS $\odot * \bullet+$ & 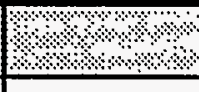 & 2 & $14 \%$ & $8,11 \%$ & $1+11$ & \%. 11. & $\%, 11 \%$ & +11 \\
\hline First line & 8 & 8 & 8 & & & & & \\
\hline General/executive & 1 & 1 & 1 & & & & & \\
\hline Projec/Program & 2 & 2 & 2 & & & & & \\
\hline \multicolumn{9}{|l|}{ other } \\
\hline \multicolumn{9}{|l|}{ Subtotal } \\
\hline ENGINEERS $\%$ & १े? & ४े०े? & $8 \%$ & \%. 62 & ४. $62 \%$ & $1 \%, 62 \%$ & 1,62, & .62 \\
\hline Chemical & 1 & 1 & 1 & & & & & \\
\hline Civil & 27 & 27 & 27 & & & & & \\
\hline \multicolumn{9}{|l|}{ Computer } \\
\hline Electrical & 10 & 10 & 10 & & & & & \\
\hline Environmental & 1 & 1 & 1 & & & & & \\
\hline \multicolumn{9}{|l|}{ Industrial } \\
\hline Mechanical & 15 & 15 & 15 & & & & & \\
\hline \multicolumn{9}{|l|}{ Nuclear } \\
\hline \multicolumn{9}{|l|}{ Petroleum/Mining } \\
\hline Plant & 5 & 5 & 5 & & & & & \\
\hline \multicolumn{9}{|l|}{ Quality Control } \\
\hline Safety & 1 & 1 & 1 & & & & & \\
\hline Other & 2 & 2 & 2 & & & & & . \\
\hline Subtotal & & & & & & & & \\
\hline
\end{tabular}




\begin{tabular}{|c|c|c|c|c|c|c|c|c|}
\hline \multicolumn{4}{|c|}{ Planned Staffing (Fu11 Time Equivalent) } & \multicolumn{5}{|c|}{ Note: Job Family Only After 1997} \\
\hline Job Famfiy & Numbers & ४े: & $\$$ & ४० : & 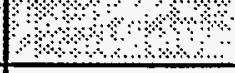 & ४ $: \%$ & ४४४ & \& \\
\hline Job Category & & 1996 & 1997 & 1998 & 1999 & 2000 & 2001 & 2002 \\
\hline SCIENTISTS & ४४ & ४४। & \%०े & ॥ै। & ४४\%?\% & ४०. .4 & ४ै४४ & ४े \\
\hline Chemists & & & & & & & & \\
\hline Environmental & & & & & & & & \\
\hline Geologists & & & & & & & & \\
\hline Life & & & & & & & & \\
\hline Material & & & & & & & & \\
\hline Mathematicians & & & & & & & & \\
\hline Physicists & & & & & & & & \\
\hline Social & & & & & & & & \\
\hline Other & & & & & & & & \\
\hline Subtotal & & & & & & & & \\
\hline $\begin{array}{l}\text { ADMINISTRATIVE /OTHER } \\
\text { PROFESSIONALS }\end{array}$ & अथे & \%ै। & $1 \%$ & $16 \%$ & ४ै $16 \%$ & \% $16 \%$ & \% $16 \%$ & 16 \\
\hline Accountant/auditor & 6 & 6 & 6 & & & & & \\
\hline Architect & 2 & 2 & 2 & & & & & \\
\hline Buyers/Procurement & & & & & & & & \\
\hline Communications & & & & & & & & \\
\hline $\begin{array}{l}\text { Compliance } \\
\text { Inspectors } \\
\end{array}$ & & & & & & & & \\
\hline $\begin{array}{l}\text { Computer Sys } \\
\text { Analyst }\end{array}$ & & & & & & & & \\
\hline $\begin{array}{l}\text { Cost Est/p1anner } \\
\text { /sch }\end{array}$ & 2 & 2 & 2 & & & & & \\
\hline
\end{tabular}




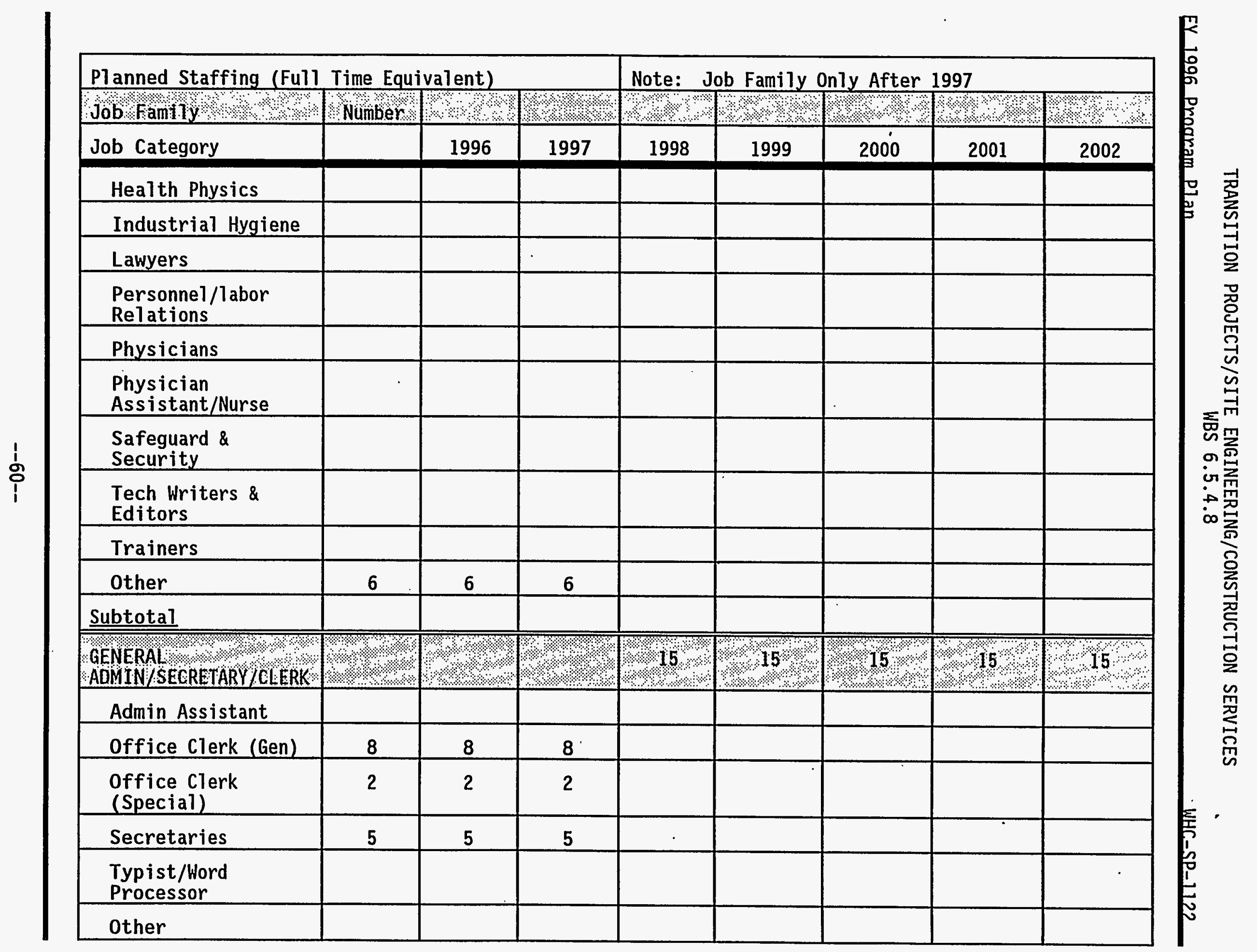




\begin{tabular}{|c|c|c|c|c|c|c|c|c|}
\hline \multicolumn{4}{|c|}{ Planned Staffing (Full Time Equivalent) } & \multicolumn{5}{|c|}{ Note: Job Family Only After 1997} \\
\hline Job Family & Number & +4) & २ি। & ४ै। & मे। & 19 १ै। & अैंद & अअ \\
\hline Job Category & & 1996 & 1997 & 1998 & 1999 & 2000 & 2001 & 2002 \\
\hline \multicolumn{9}{|l|}{ Subtotal } \\
\hline TECHNICIANS & 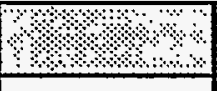 & ४४४, & \%०ै? & \%ै। & $+34 \%$ & $18 \% 34 \%$ & \% $34 \%$ & \% $34 \%$ \\
\hline \multicolumn{9}{|l|}{$\begin{array}{l}\text { Computer } \\
\text { Operator/Coder }\end{array}$} \\
\hline Drafters & 33 & 33 & 33 & & & & & \\
\hline Engineers/Technicians & 1 & 1 & 1 & & & & & \\
\hline \multicolumn{9}{|l|}{$\begin{array}{l}\text { Environmental } \\
\text { Science } \\
\text { Technicians } \\
\end{array}$} \\
\hline \multicolumn{9}{|l|}{$\begin{array}{l}\text { Health Physics } \\
\text { Technicians }\end{array}$} \\
\hline \multicolumn{9}{|l|}{$\begin{array}{l}\text { Industrial } \\
\text { Saf/health } \\
\text { Technicians }\end{array}$} \\
\hline \multicolumn{9}{|l|}{$\begin{array}{l}\text { Instru/Control } \\
\text { Technicians }\end{array}$} \\
\hline \multicolumn{9}{|l|}{ Lab. Technicians } \\
\hline \multicolumn{9}{|l|}{ Media Technicians } \\
\hline \multicolumn{9}{|l|}{$\begin{array}{l}\text { Survey/Map } \\
\text { Technicians }\end{array}$} \\
\hline \multicolumn{9}{|l|}{ other } \\
\hline \multicolumn{9}{|l|}{ Subtotal } \\
\hline GRAFTS & 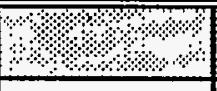 & $\% \% \%$ & \%४े?: & \%ै। & अै. & ४ै\% & +. & e \\
\hline \multicolumn{9}{|l|}{ Carpenters } \\
\hline Electricians & & & & & & & & \\
\hline
\end{tabular}




\begin{tabular}{|c|c|c|c|c|c|c|c|c|}
\hline \multicolumn{4}{|c|}{ Planned Staffing (Full Time Equivalent) } & \multicolumn{5}{|c|}{ Note: Job Family Only After 1997} \\
\hline Job ramny & Number? & 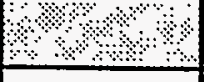 & \%॰ \% & $\because 8$ & ४ै४ & ४े? & एా? & ४ै। \\
\hline Job Category & & 1996 & 1997 & 1998 & 1999 & 2000 & 2001 & 2002 \\
\hline \multicolumn{9}{|l|}{ HVAC } \\
\hline \multicolumn{9}{|l|}{ Machinists } \\
\hline \multicolumn{9}{|l|}{ Masons } \\
\hline \multicolumn{9}{|l|}{ Millwrights } \\
\hline \multicolumn{9}{|l|}{ Painters } \\
\hline \multicolumn{9}{|l|}{ Plmbrs/Pipefitters } \\
\hline \multicolumn{9}{|l|}{$\begin{array}{l}\text { Struct/Metal } \\
\text { Workers }\end{array}$} \\
\hline \multicolumn{9}{|l|}{$\begin{array}{l}\text { Vehicl/Mobile } \\
\text { Equip. } \\
\text { Mechanics }\end{array}$} \\
\hline \multicolumn{9}{|l|}{ Welders } \\
\hline \multicolumn{9}{|l|}{ other } \\
\hline \multicolumn{9}{|l|}{ Subtotal } \\
\hline OPERATORS $\%$ । $\%$ & \%? & २०े? & $\%$ & ४े. & \%ै\%? & ४४४\% & ४ै। & अै। \\
\hline \multicolumn{9}{|l|}{ Lt. Vehicle Drivers } \\
\hline \multicolumn{9}{|l|}{$\begin{array}{l}\text { Material Moving } \\
\text { Equip. }\end{array}$} \\
\hline \multicolumn{9}{|l|}{ Nuclear Plant } \\
\hline \multicolumn{9}{|l|}{$\begin{array}{l}\text { Utilities Waste } \\
\text { Proces }\end{array}$} \\
\hline \multicolumn{9}{|l|}{ Other } \\
\hline Subtotal & & & & & & & & \\
\hline
\end{tabular}




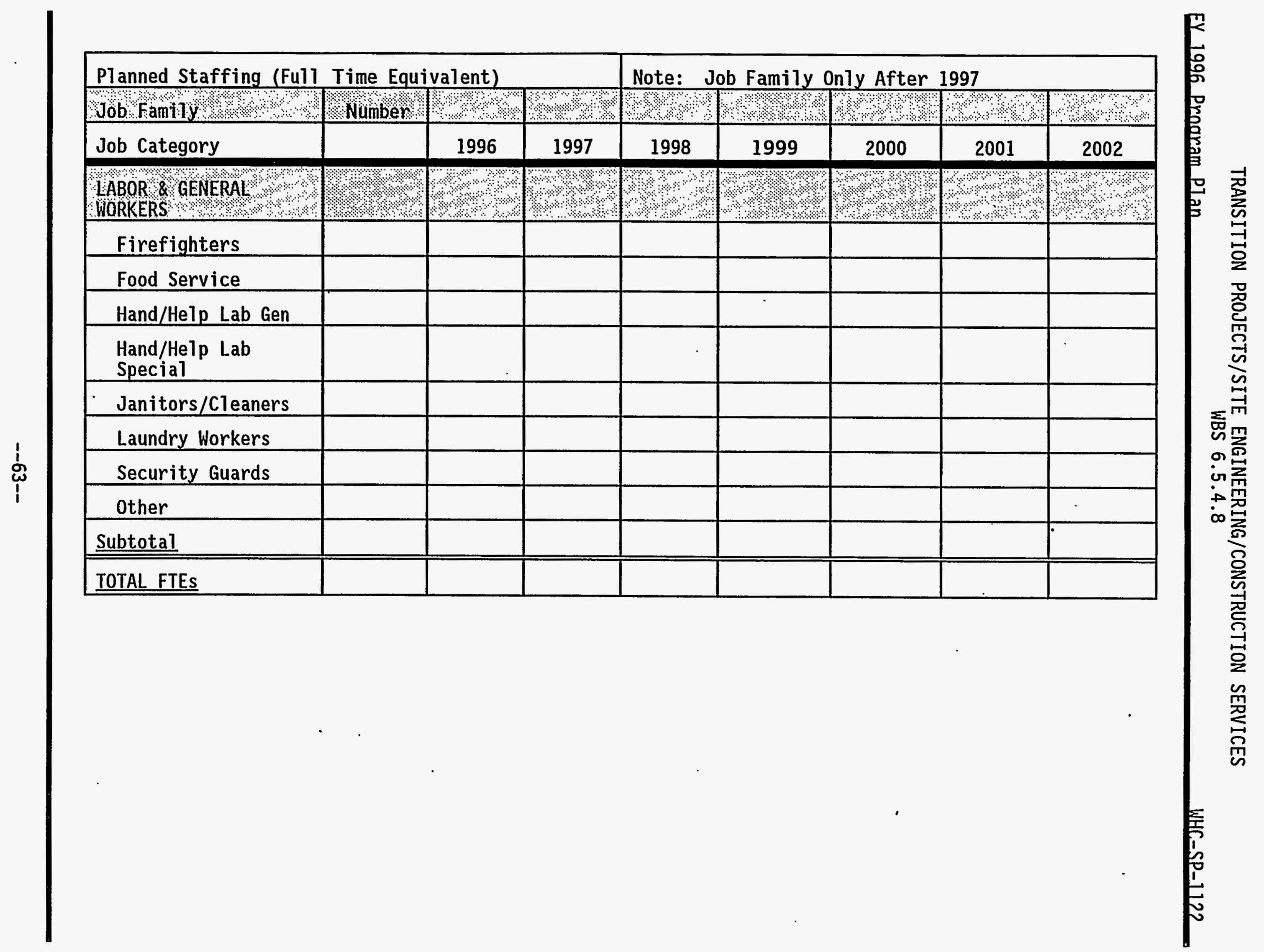




\section{DIRECT POOL - NON-MANUAL}

WBS 6.5 .4

FY 1996 Program PTan

.0 SCHEDULE BASELINE

4.1 Product Deliverables/Milestone List ----Not App T icable---- 


\section{DIRECT POOL - NON-MANUAL}

HBS 6.5 .4

FY 1996 Program Plan

WHC-SP-1122

.0 COST BASELINE SUMMARY

5.1 Cost Baseline

5.2 Planned Staffing Profile 
ICF Kaiser Hanford

Non-ManualPool

ProposedFY 96 Budget

(In Thousands)

\begin{tabular}{|c|c|c|c|}
\hline & $\begin{array}{c}\text { FY96 } \\
\text { BUDGET }\end{array}$ & $\begin{array}{l}\text { FY } 95 \\
\text { FYSF }\end{array}$ & $\begin{array}{c}\text { ORIGINAL } \\
\text { FY } 95 \\
\text { PLAN } \\
\end{array}$ \\
\hline $\begin{array}{l}\text { Direct Burdened Labor } \\
\text { Indirect BurdenedLabor }\end{array}$ & $\begin{array}{l}44,681 \\
11,974\end{array}$ & $\begin{array}{l}44,966 \\
13,169\end{array}$ & $\begin{array}{l}61,787 \\
13,536\end{array}$ \\
\hline Total LABOR & 56,655 & 58,135 & 75,323 \\
\hline Nonlaborcosts & 13,306 & 18,119 & 19,902 \\
\hline Total Budget & 69,961 & 76,254 & 95,225 \\
\hline FTE'S & 776 & 841 & 1,112 \\
\hline Rate/Adder & $51.95 \%$ & $66.00 \%$ & $49.70 \%$ \\
\hline G\&A & $13 \%$ & $17 \%$ & $17 \%$ \\
\hline TOTALADDER & $72 \%$ & $94 \%$ & $75 \%$ \\
\hline
\end{tabular}




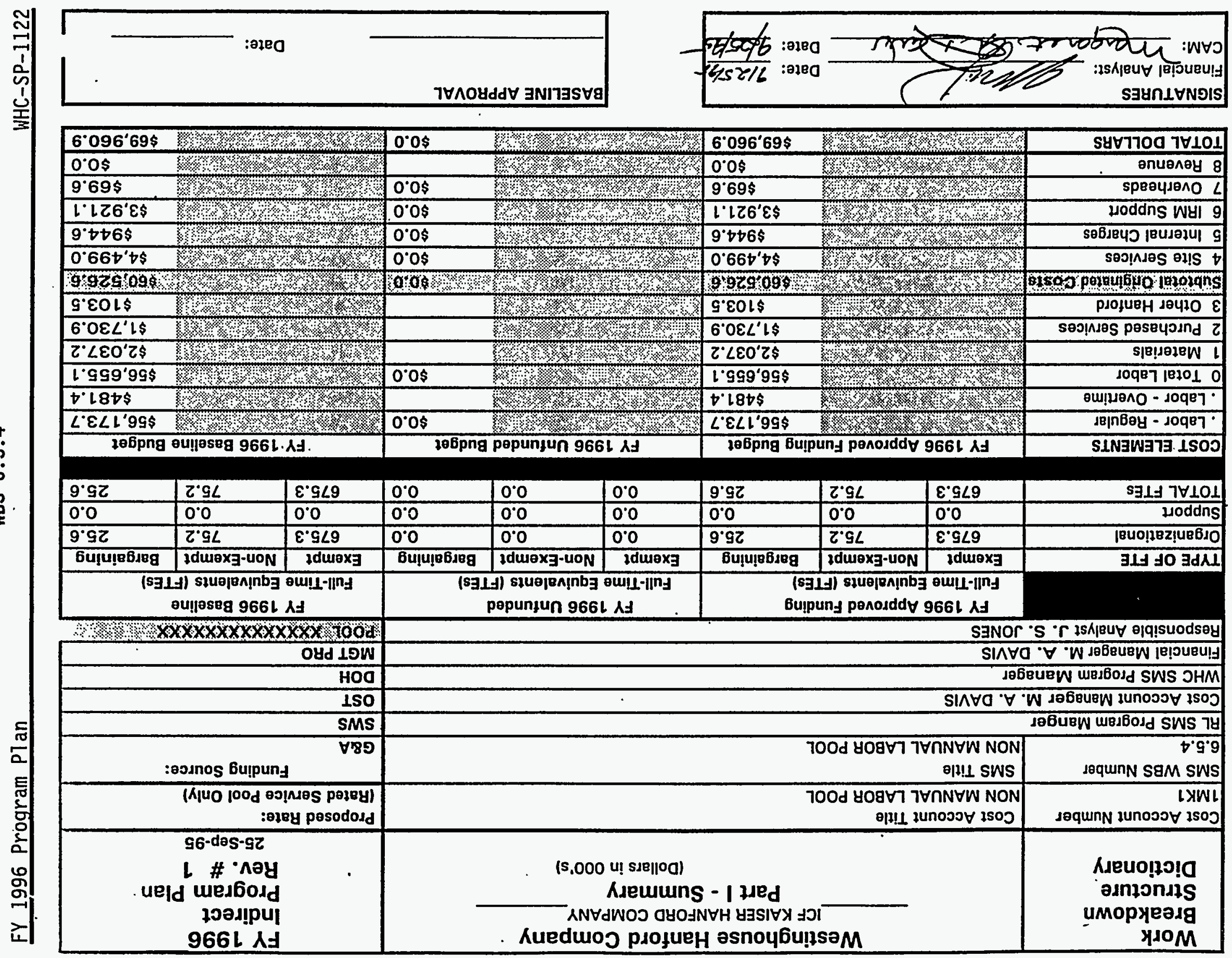




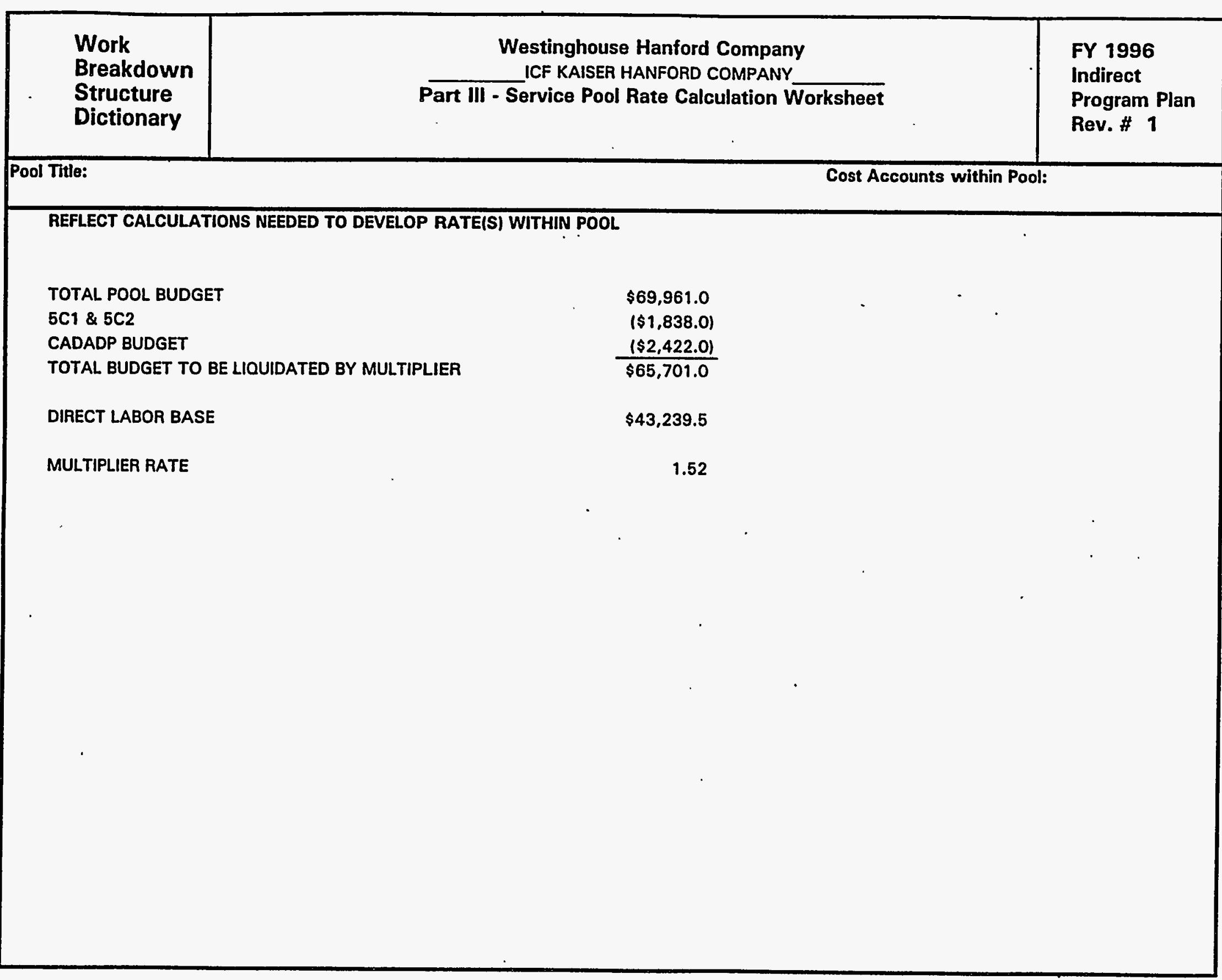




\begin{tabular}{|c|c|c|c|c|c|c|c|c|}
\hline \multicolumn{4}{|c|}{ Planned Staffing (Full Time Equivalent) } & \multicolumn{5}{|c|}{ Note: Job Family Only After 1997} \\
\hline Job Eamily $\rightarrow$ \% & Number & 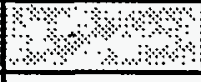 & 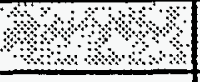 & : & ४४४: & 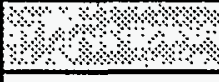 & \%? & ४ै। \\
\hline Job Category & & 1996 & 1997 & 1998 & 1999 & 2000 & 2001 & 2002 \\
\hline MANAGERS & +1 & ? & $8 \%$ \%? & $1 \% 67$ & $8.67 \%$ & \% $67 \%$ & 2.67 & $\% 67$ \\
\hline First line & 54 & 54 & 54 & & & & & \\
\hline General/executive & 7 & 7 & 7 & & & & & \\
\hline Projec/Program & 6 & 6 & 6 & & & & & \\
\hline other & & & & & & & & \\
\hline Subtotal & & & & & & & & \\
\hline ENGINEERS & थ००.? & थे. & \%थ थ & 354 & ४२ $354 \div$ & (\%) 354 & ४3.54\% & $\ll 354$ \\
\hline Chemical & 11 & 11 & 11 & & & & & \\
\hline Civil & 103 & 103 & 103 & & & & & \\
\hline Computer & 1 & 1 & 1 & & & & & \\
\hline Electrical & 56 & 56 & 56 & & & & & \\
\hline Environmental & 4 & 4 & 4 & & & & & \\
\hline Industrial & 6 & 6 & 6 & & & & & \\
\hline Mechanical & 89 & 89 & 89 & & & & & \\
\hline Nuclear & 1 & 1 & 1 & & & & & \\
\hline Petroleum/Mining & & & & & & & & \\
\hline Plant & 41 & 41 & 41 & & & & & \\
\hline Qual ity Control & 1 & 1 & 1 & & & & & \\
\hline Safety & 10 & 10 & 10 & & & & & \\
\hline Other & 31 & 31 & 31 & & & & & \\
\hline Subtotal & & & & & & & & \\
\hline
\end{tabular}




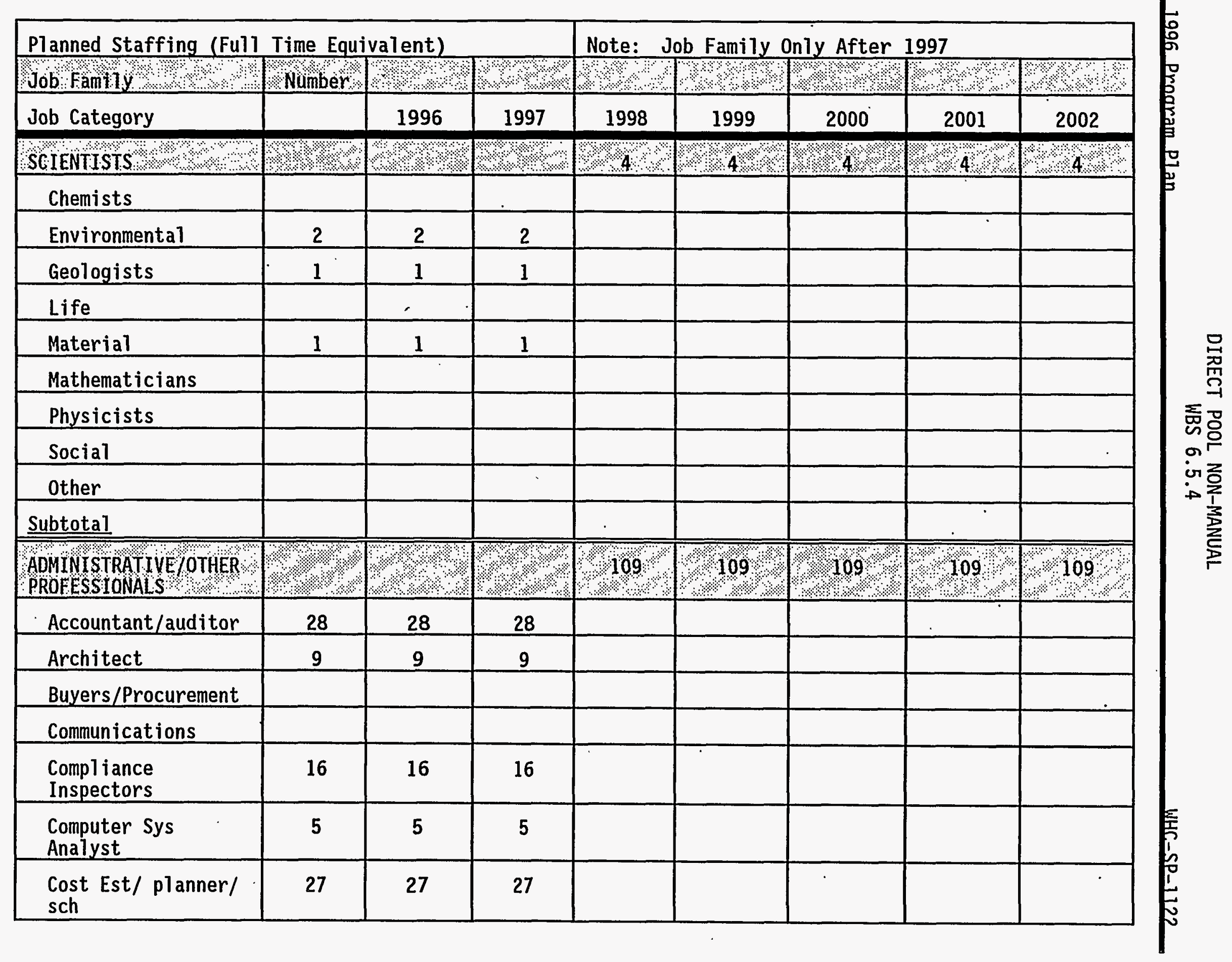




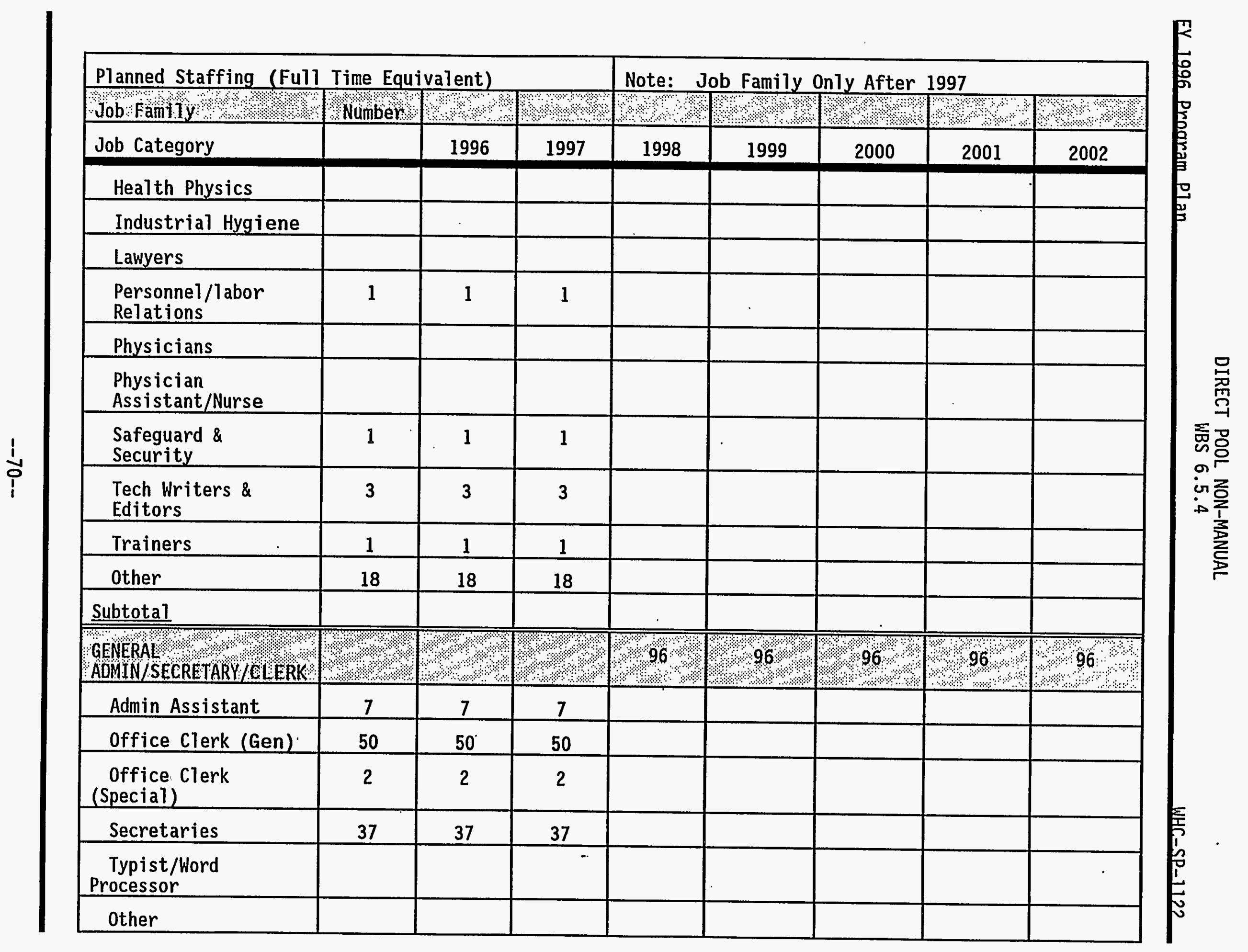




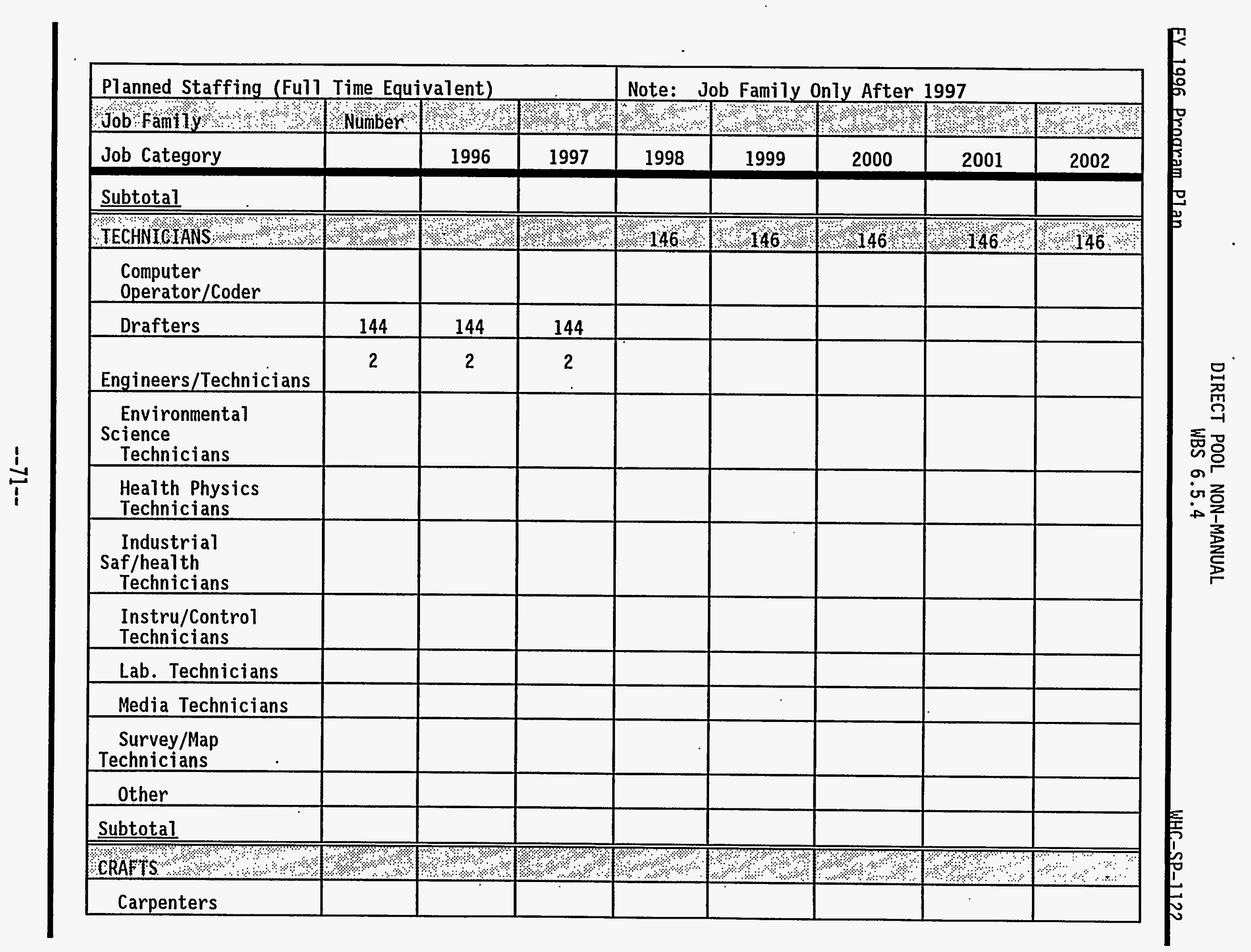




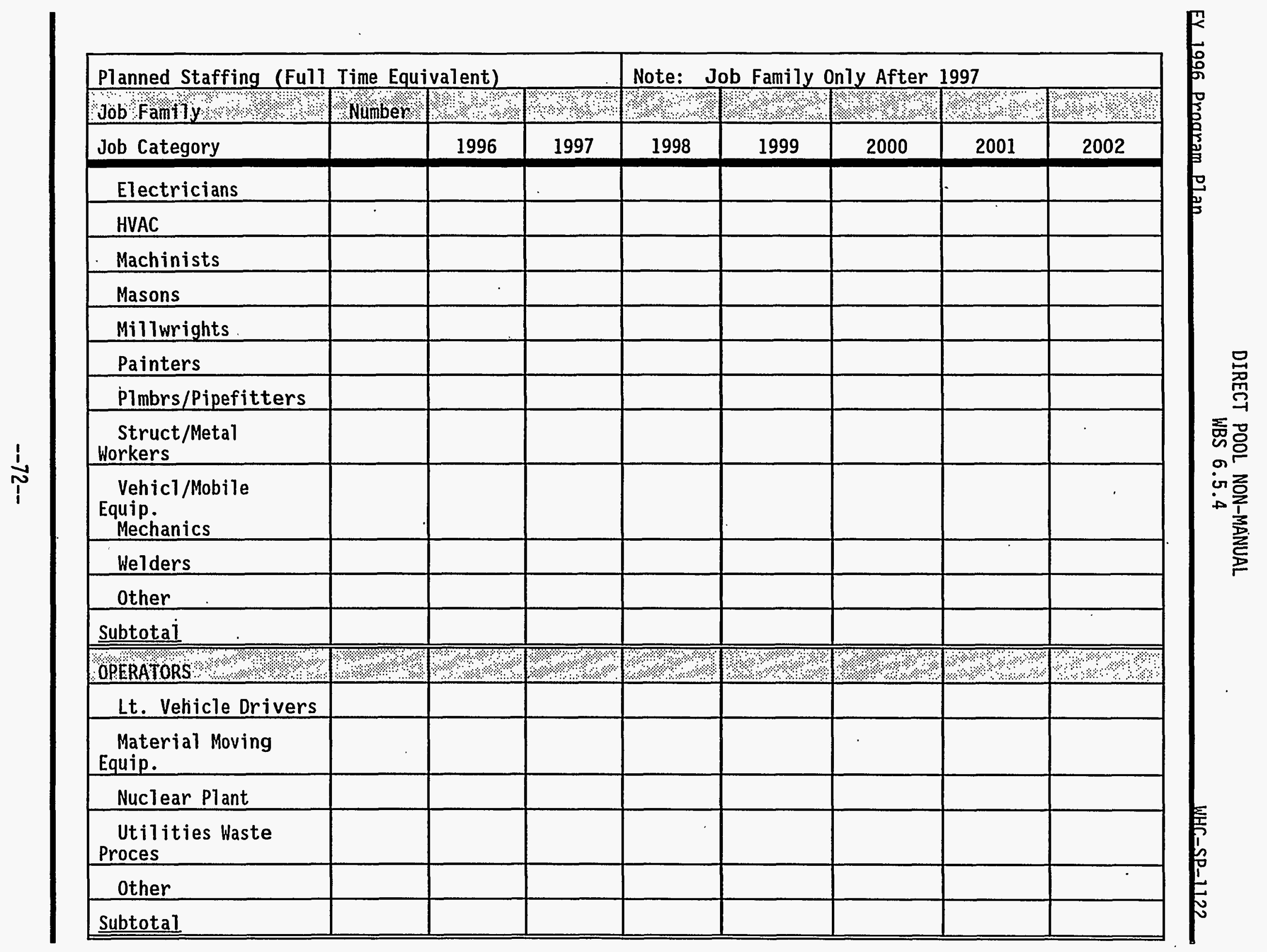




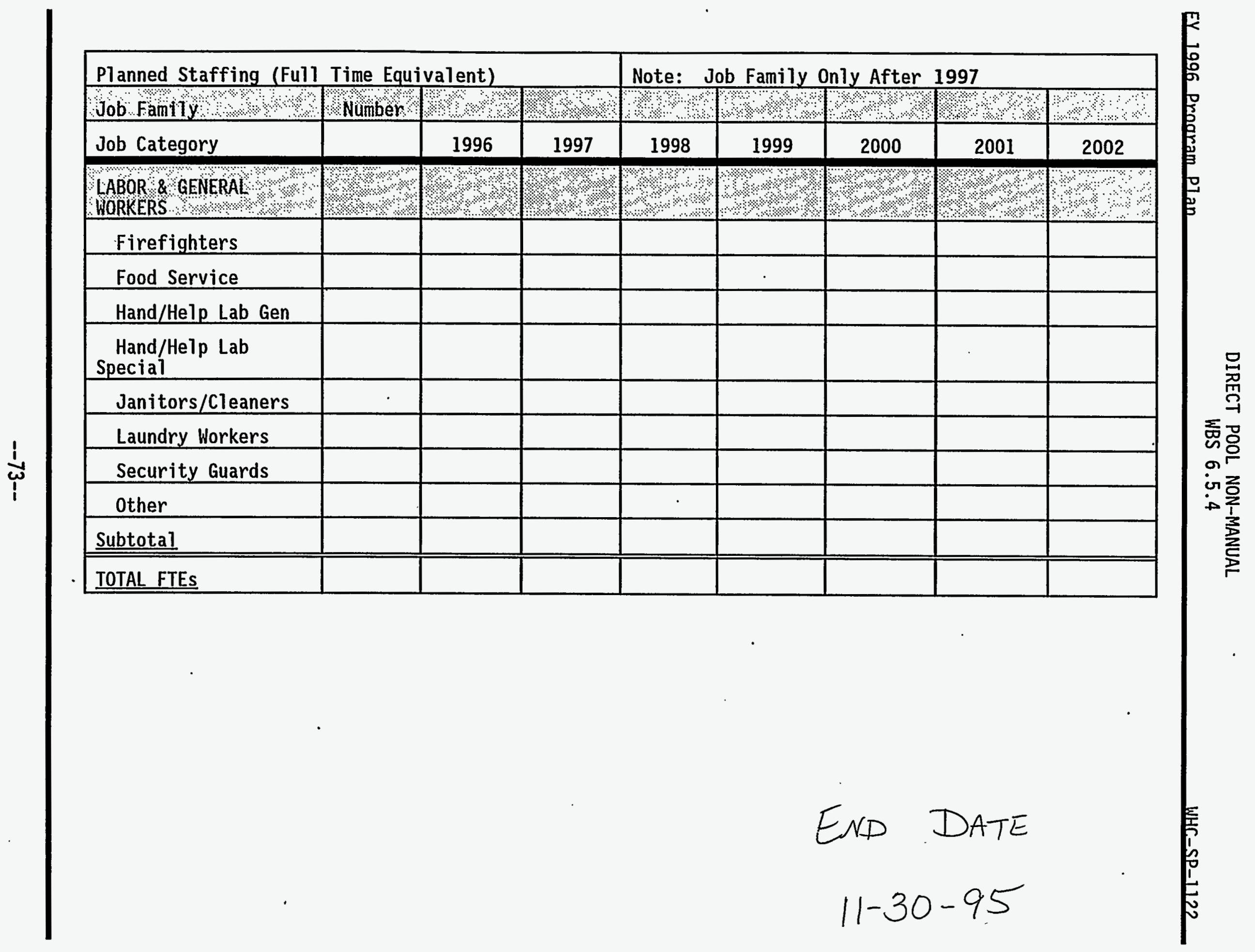

